



\section{NIST Technical Note 1547}

\section{Electromagnetic Airframe Penetration \\ Measurements for the FAA Bombardier Global 5000}

Chriss Grosvenor, David Novotny, Dennis Camell, and Galen Koepke Electromagnetics Division

Electromagnetics and Electrical Engineering Laboratory

National Institute of Standards and Technology

Boulder, CO 80305-3328

Robert T. Johnk

National Telecommunication and Information Agency

Institute for Telecommunications Sciences

Boulder, CO 80305-3328

June 2008

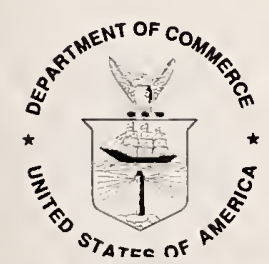

U.S. Department of Commerce

Carlos M. Gutierrez, Secretary

National Institute of Standards and Technology

James M. Turner, Deputy Director 
National Institute of Standards and Technology

Technical Note 1547

Natl. Inst. Stand. Technol.

Tech. Note 1547

XX pages (Date 2008)

CODEN: NTNOEF
U.S. Government Printing Office

Washington: 2008

For Sale by the

Superintendent of Documents

U.S. Government Printing Office

Stop SSOP, Washington, DC 20402-0001

Phone: (202) 512-1800

Fax: (202) 512-2250

Internet: bookstore.gpo.gov 


\section{Contents}

1. History and Background. . . . . . . . . . . . . . . . . . . . 1

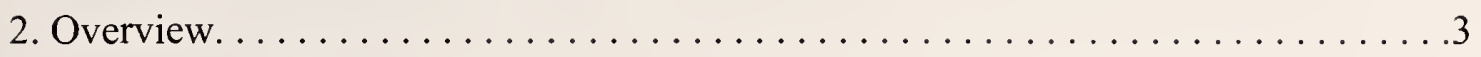

3. Measurement System. . . . . . . . . . . . . . . . . . . . 6

4. Measurement of Electromagnetic Airframe Penetration $\ldots \ldots \ldots \ldots \ldots \ldots$

5. Reference Measurements. ............................ 12

6. Airframe Penetration Measurements — Overview. ................ 18

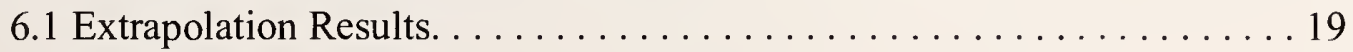

6.2 Main Passenger Cabin Penetration Results................21

6.3 Flight Deck Penetration Results. ..................... 44

6.4 Cargo Closet Penetration Results. . . . . . . . . . . . . . . 61

6.5 HIRF Positions for Flight Deck Penetration. . . . . . . . . . . . . 79

6.6 Internal Coupling Measurements. . . . . . . . . . . . . 83

7. Uncertainty Analysis. . . . . . . . . . . . . . . . . . . . . . 90

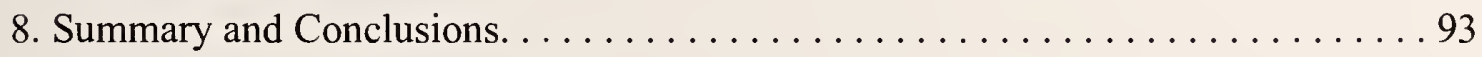

9. References. . . . . . . . . . . . . . . . . . . . . . . . . . . . . .97

Appendix A. Time Domain Waveform Analysis. . . . . . . . . . . . 98

Appendix B. Signal and Noise Characteristics. . . . . . . . . . . . . . 109

Appendix C. TEM and DRG Horn Boresight Gain Characteristics. ...... 112

Appendix D. Penetration Data for HIRF Frequency Bands. . . . . . . . . . . 114

Appendix E. Bombardier Global 5000 Measurement Equipment List. . . . . . 119 


\title{
Electromagnetic Airframe Penetration Measurements for the FAA Bombardier Global 5000
}

\author{
Chriss Grosvenor, David Novotny, Dennis Camell, and Galen Koepke \\ Electromagnetics Division \\ National Institute of Standards and Technology \\ 325 Broadway \\ Boulder, CO 80305 \\ and \\ Robert T. Johnk
National Telecommunication and Information Agency
Institute for Telecommunication Sciences
325 Broadway
Boulder, CO 80305

The National Institute of Standards and Technology has recently completed shielding effectiveness/penetration studies on three different aircraft types for the Federal Aviation Administration. The studies are used to understand the cavity coupling characteristics between antennas placed in various compartments inside the aircraft and antennas placed at various angular positions around an aircraft. This document shows how penetration varies as a function of frequency, antenna type, antenna polarization, and cavity susceptibility for a Bombardier Global 5000 business jet. Internal coupling between two antennas placed in the aircraft at different locations to determine the quality factor and the time decay of fields is also presented. These measurements will provide the Federal Aviation Administration with a database on penetration for three different classes of aircraft.

Key words: aircraft; cavity coupling; digital signal processing; HIRF; penetration; shielding effectiveness; synthetic time-domain measurements.

\section{History and Background}

The Field Parameters and EMC Applications Project of the National Institute of Standards and Technology (NIST) has recently completed shielding effectiveness studies of three representative aircraft for the Federal Aviation Administration (FAA). These studies will be used to provide the FAA with the procedures and data reduction techniques for typical low-level airframe penetration for high intensity radiated field (HIRF) attenuation/shielding tests. This technical note addresses aircraft shielding tests conducted on a Bombardier Global 5000 business jet owned and operated by the FAA. Two additional reports will detail studies of (1) a 737-200 owned and operated by the FAA and (2) a Beechcraft Premier IA owned by the Hawker- 
Beechcraft Corporation. The 737-200 represents a typical commercial aircraft, and the Premier IA represents a typical carbon-composite aircraft. By measuring all three aircraft and comparing them, we have the potential to provide a "design roadmap" for the optimization of HIRF testing standards for the electromagnetic compatibility (EMC) aircraft manufacturing community.

The FAA currently has specific certification policies addressing the effects of HIRF on an aircraft's electrical and electronic systems that have been applied for the past 19 years [1]. In the FAA document, the regulatory authorities and industry have defined HIRF environments, requirements for aircraft HIRF protection, and methods for testing and verifying the level of HIRF protection. Most of reference [1] discusses HIRF testing for electronic and electrical subsystems, which this technical note does not address. In this technical note, we specifically address both high- and low-level airframe shielding compliance testing. The HIRF procedures are found in Section 6.6.2.6 and Section 6.6.4 of Reference [1].

The basic problem is to evaluate the coupling of an external source into a large, leaky cavity. The cavity could represent the whole aircraft or a zone (cargo bay, flight deck, etc.) depending on the expected vulnerability. In a typical HIRF measurement, the measurement system is calibrated and a reference measurement is taken without the aircraft embedded in the test setup. The aircraft is then placed into the test setup and various measurements are taken in susceptible cavities. These attenuation tests are typically conducted using either mechanical stirring or frequency stirring methods [2,3]. The airframe attenuation is calculated by use of the following equation,

$$
A_{\text {atten }}=S . E .=\frac{\text { reference measurement }}{\text { cavity measurement }},
$$

where $\mathrm{A}_{\text {atten }}$ is the airframe attenuation and S.E. is defined as the shielding effectiveness. These numbers, expressed in decibels, are typically positive so that the more positive the number, the greater the protection the aircraft provides to electromagnetic fields, and as the number approaches zero we expect almost no shielding protection. The aircraft HIRF attenuation data vary as a function of frequency, physical position of the measurement antenna, characteristics of the aircraft, and configuration of the test site. After a company tests and processes the data, the information is given to the FAA, which uses it, in conjunction with the subsystem susceptibility tests to determine an aircraft's HIRF vulnerability.

The detailed method used to process aircraft HIRF attenuation data is not well documented and is closely held by the handful of test service providers. Therefore, the interpretation of the data is not immediately obvious to the certification engineer. This technical note and the two to follow will consider attenuation measurements as a function of frequency, antenna position, antenna type, and cavity selection. Our data processing methodology will be shown in detail. Using this information, we hope to provide guidelines for both the FAA and test engineers to develop a uniform and transparent test method for data processing and documentation. We know that the confidence levels are expected to be dependent on the number of sample points 
(measurement geometries), but we should be able to assess the trade-offs between measurement effort (sample number) and risk (confidence level).

\section{Overview}

This report summarizes results of a measurement effort conducted by the Field Parameters and EMC Applications Project team of NIST. This effort consisted of an extensive series of penetration measurements performed on a Bombardier Global 5000 owned and operated by the FAA. The aircraft was tested at the FAA site in Atlantic City, New Jersey during the period from October 3, 2005 to October 8, 2005. The purpose of this effort is to provide the FAA with a database of penetration data on the Bombardier Global 5000, which is a commercial business jet.

The effort utilized NIST-developed measurement systems consisting of ultra-wideband transverse electromagnetic (TEM) horn antennas, 3117 dual-ridged guided (DRG) horn antennas, a commercially available vector network analyzer (VNA), interconnecting transmission media, and an amplifier. Interconnections between transmitting and receiving antennas were made using $11 \mathrm{GHz}$ and $18 \mathrm{GHz}$ precision, analog electro-optic links. This effort was the first time NIST researchers used a broadband, analog, electro-optic link in conjunction with a configurable VNA and amplifier for airframe shielding studies up to $18 \mathrm{GHz}$. Shielding data are obtained from a direct comparison of two measurements: (1) a transmission measurement between boresighted antennas located outside the aircraft to obtain a reference, and (2) transmission measurements with one antenna located inside a selected compartment of the aircraft and a pair of horizontally and vertically polarized antennas for both low-frequency-band and highfrequency-band antennas located outside the aircraft at specified locations. Shielding data are obtained by comparing processed reference and aircraft transmission data. This is achieved using an efficient sequence of Fourier and inverse Fourier transformations, frequency domain convolutions, and filtering combined with time gating and frequency averaging. Shielding values are obtained by taking the ratio of the gated amplitude spectra of the aircraft measurement and the gated amplitude spectra of the references. Data smoothing is performed using the frequency-averaging of signal power over a specified bandwidth as described in [2]:

$$
\left\langle S E\left(f_{n}\right)\right\rangle=\frac{1}{2 N+1} \sum_{i=n-N}^{n+N}\left|S E\left(f_{i}\right)\right|^{2}
$$

Shielding data were obtained with a receiving antenna located in one of three internal compartments: (1) the main passenger cabin, (2) the flight deck, and (3) the internal aft cargo closet. A pair of transmitting antennas was positioned outside the aircraft at a fixed height of 4 $\mathrm{m}$, which is the approximate height of the windows, at a fixed distance of $23 \mathrm{~m}$ from the center of the aircraft. The transmitting antennas were boresighted at the center of the aircraft, and data were obtained for both the horizontal and vertical polarizations. We based our tests on HIRF standard test procedures and other questions we felt were important to ask. These standards are concerned with detecting possible leakage into the aircraft. Key areas include the flight deck, the avionics bay, any windows/doors/joints, and the wing/tail section of the fuselage. We defined a test setup at various angular positions around the aircraft, around the flight deck of the aircraft at five positions at a constant distance from the front landing gear of the aircraft, and internal 
coupling measurements. In a recent paper [4], questions were raised as to how many positions need to be measured around an electrically large object to fully characterize the fields penetrating into the aircraft for a particular high-frequency limit. For this reason, we designed the test plan as follows: (1) From $0^{\circ}$ to $120^{\circ}$, measurements were conducted every $10^{\circ}$; (2) from $120^{\circ}$ to $160^{\circ}$ and from $330^{\circ}$ to $360^{\circ}$, measurements were carried out in $5^{\circ}$ increments; and (3) additional measurements were carried out at $190^{\circ}$ and $270^{\circ}$ in order to look at symmetry. Finally, we concluded with a set of internal coupling measurements to understand the reverberant environment internal to the aircraft. An aircraft's level of electromagnetic field protection, referred to as shielding, depends on the level of treatment, the compartment, the frequency, the transmitting antenna polarization, and the angle of illumination. In this document, we will display penetration values, which are the reciprocal of shielding values. On the graphs that follow, a penetration near $0 \mathrm{~dB}$ means electromagnetic fields can easily penetrate into that part of the aircraft. Penetration levels for the Global 5000 Bombardier range from approximately $0 \mathrm{~dB}$ to $-45 \mathrm{~dB}$. The estimated dynamic range of this system is $50 \mathrm{~dB}$ at $4000 \mathrm{MHz}$ and $30 \mathrm{~dB}$ at $18,000 \mathrm{MHz}$. Maximum penetration results were obtained with the receiving antenna in the main passenger cabin, while lower values were obtained in the flight deck and the aft cargo closet. Maximum penetration in the main passenger cabin was observed with the transmitting antennas illuminating the aircraft from positions located at the side of the aircraft. Minimum penetration was seen in the aft cargo closet at both the nose and tail positions.

This report is divided into five main sections: (1) a description of the measurement system, (2) an overview of the measurement technique, (3) an overview of the signal processing, (4) a summary of results for the three different aircraft compartments and various internal measurements, and (5) an uncertainty analysis. Five appendices discuss the impact of time gating, signal and noise, an equipment list, maximum penetration as a function of the HIRF specified frequency bands, and the gain characteristics of the ultra-wideband antennas that were used. 


\section{Measurement System}

The configuration of the NIST measurement system for these aircraft measurements is shown in Figures 1 and 2. The system consists of: (1) a VNA, (2) two transmitting TEM horn antennas and two DRG horn antennas configured for the horizontal and vertical polarizations, respectively, (3) a receiving TEM horn antenna and DRG antenna, (4) two coaxial microwave switches to select the polarization of the transmit antennas, and (5) two precision analog electrooptic links to cover both frequency bands and to provide an interconnection between the transmitting antennas outside the aircraft and the receiving antenna inside. These links provide a significant improvement in performance over conventional microwave cables. The optical links result in low transmission losses and low noise, and they do not pick up common-mode noise from environmental ambient signals. The optical links also provide an improvement in dynamic range and have better immunity from electromagnetic interference. The heart of this system is a commercially available four-port VNA that has been configured to acquire complex transmission data ( $\mathrm{S}_{31}$ for the TEM antennas and $\mathrm{S}_{42}$ for the DRG antennas, in-phase and quadrature signals). Due to the internal hardware, the VNA was limited to a maximum of 16001 frequencies, but we developed software that extends the number of frequencies to any desired value. If we need more than 16001 points, the software subdivides a given frequency range into a user-selected number of 16001 point sub-bands. The DRG horn antennas operate from $1 \mathrm{GHz}$ to $18 \mathrm{GHz}$ and were configured using two bands, resulting in 32002 equally spaced measurement points from approximately $0.5 \mathrm{MHz}$ to $18 \mathrm{GHz}$. The TEM horn antennas operate from $0.1 \mathrm{GHz}$ to $4 \mathrm{GHz}$ and were configured using one band, resulting in 6401 equally spaced measurement points from

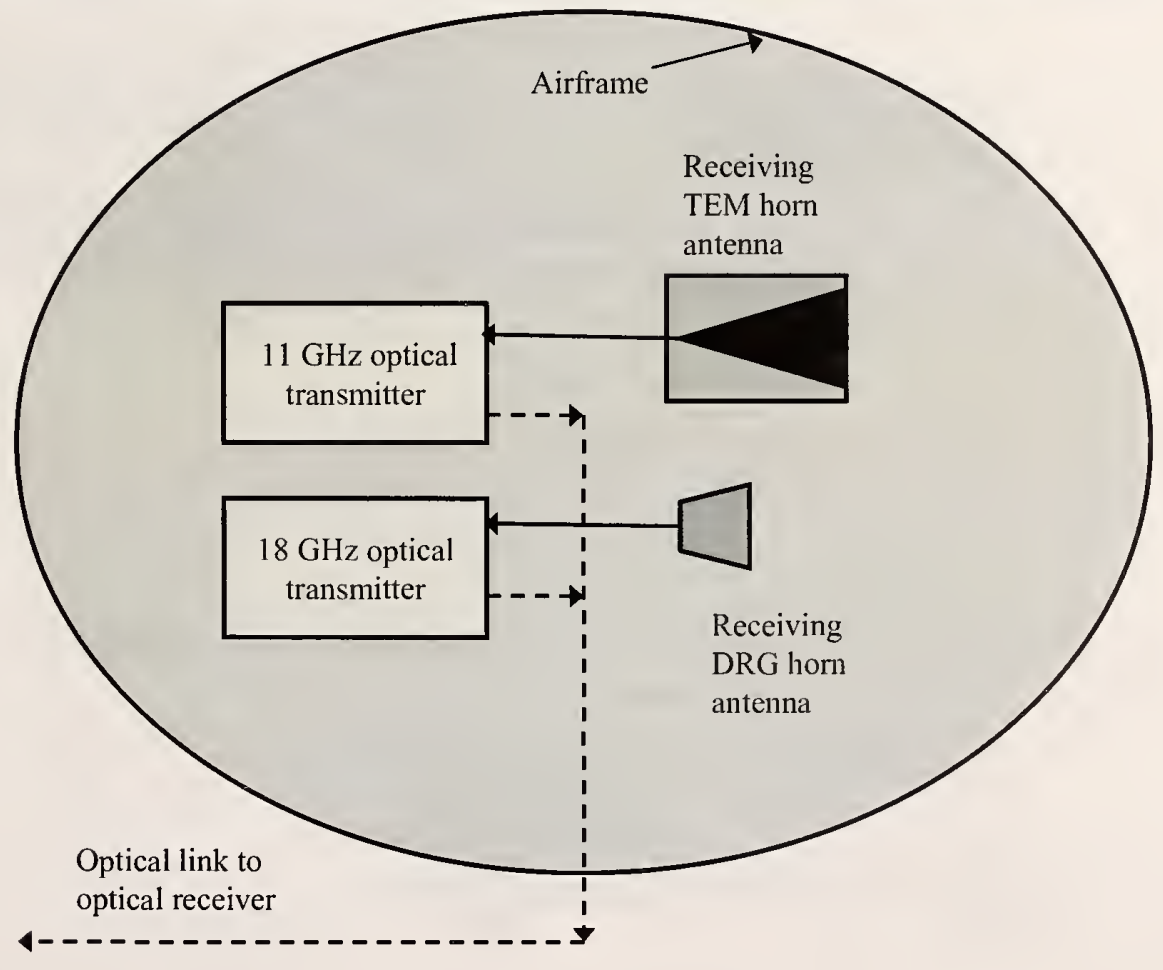

Figure 1. Airframe shielding measurement system (receiving side). The reverberant nature of the airframe permits the reception of both horizontally and vertically polarized signals. 
approximately $0.5 \mathrm{MHz}$ to $4 \mathrm{GHz}$. A reduced frequency spacing is necessary to avoid problems due to aliasing. Prior to connecting the cables to the antennas, a calibration of all channels was performed to remove systematic transmission effects due to cabling, switches, and the frequency and phase variations of the optical transmission link. This procedure calibrates the system with respect to the input of the transmitting and receiving antennas. Three NIST-developed ultrawideband TEM horn antennas were used: two $1.2 \mathrm{~m}$ antennas on a tower located outside of the aircraft, either to transmit horizontally and vertically polarized fields, and a more compact $36 \mathrm{~cm}$ TEM horn located in a pre-selected aircraft compartment to detect energy coupled into the airframe. For the higher frequency bands, three commercially available DRG antennas were used: two on the same transmitting tower located outside of the aircraft to transmit horizontally and vertically polarized fields, and a DRG antenna located in the same preselected aircraft compartment to detect energy coupled into the airframe. The frequency data were digitized and transferred to a laptop computer for subsequent data analysis and signal processing.

\section{Measurement of Electromagnetic Airframe Penetration}

The extraction of airframe penetration characteristics requires a two-step measurement process, shown in Figure 3. In the first step, a reference transmission measurement is performed by

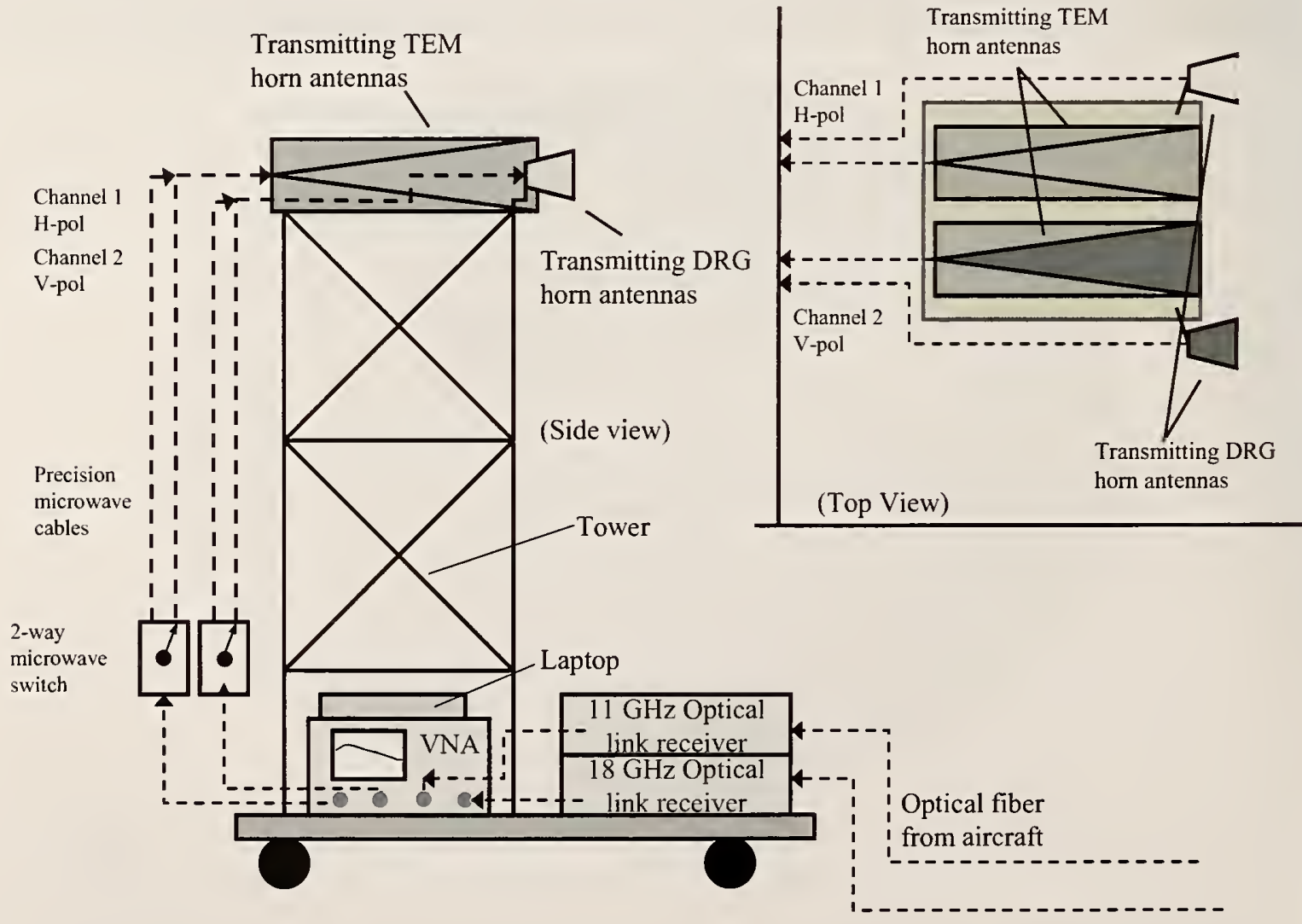

Figure 2. Transmitting side of the airframe shielding measurement system. 
boresighting the transmitting and receiving antennas at a fixed distance. The reference measurements were performed at a location away from the aircraft in order to minimize reflections from the aircraft. This measurement quantifies the energy incident on the aircraft, and enables us to calibrate and remove the frequency-dependent effects of the antennas and interconnecting hardware. In the second step, the receiving antenna was placed in a selected aircraft compartment; the transmitting antennas were placed at fixed locations, external to the aircraft, and stepped-frequency transmission data were acquired.

The reference and airframe data were processed using a sequence of filtering, Fourier transforms, and time gating to obtain the penetration values. The basic process through which the data were obtained is shown in Figure 4. The raw frequency data were first filtered to maximize the signalto-noise ratio and to minimize effects from the low-frequency cut-off of our electro-optic link. Next, a tapered frequency-domain window was applied to both sets of data to reduce Gibbs ringing in subsequent processing. An inverse Fourier transform (IFT) was then applied to the processed data to obtain time-domain waveforms. Time gates were then applied to isolate the desired portions of waveforms and remove undesired portions. In the case of the reference, the time gate was applied to isolate the direct antenna-to-antenna coupling and remove the effects of ground bounce and late-time reflections. Time gating was applied to the airframe data to isolate either the direct illumination or reverberant fields of the aircraft. Effects from ground bounce are embedded in the waveforms at this distance and therefore cannot be removed by time-gating. The time-gated data sets were then transformed back into the frequency domain, and the
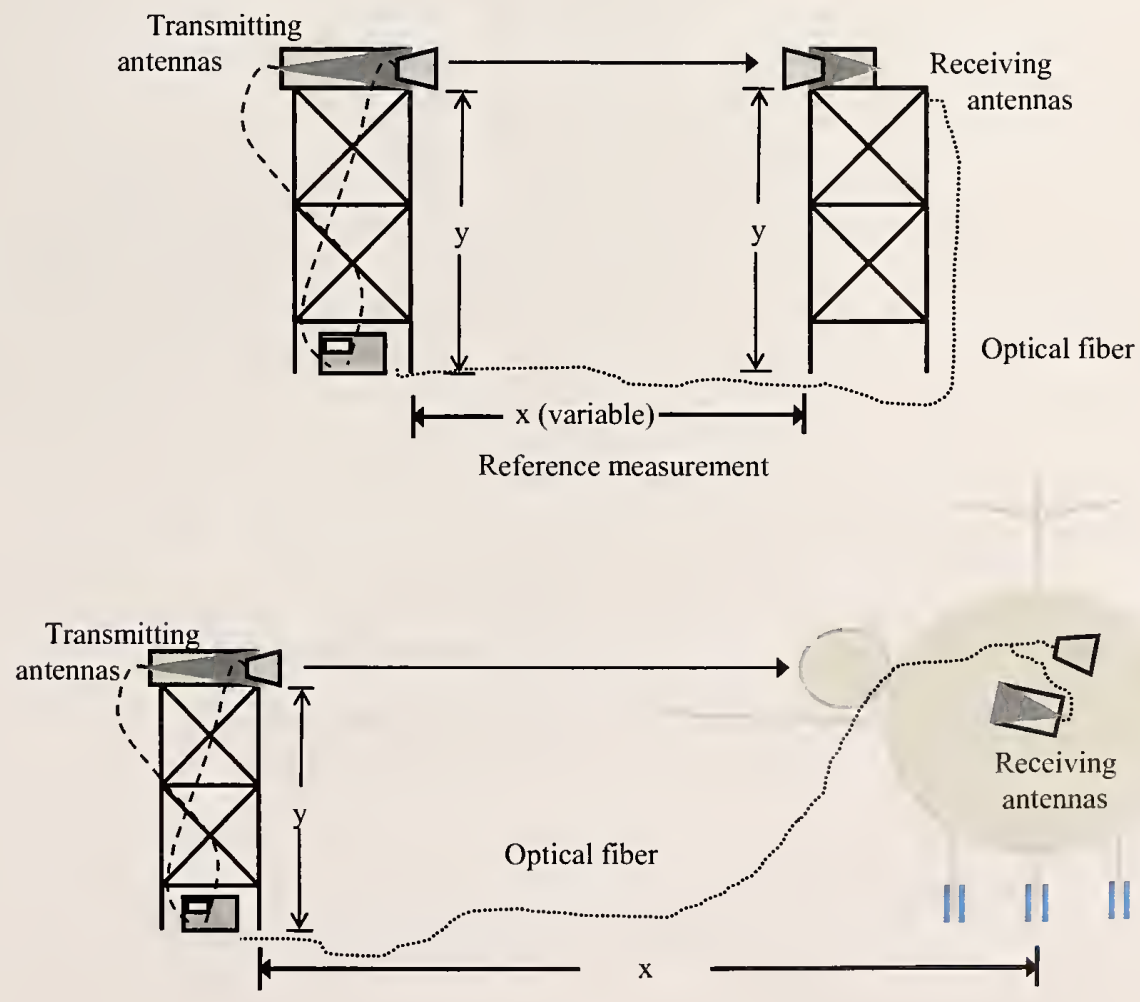

Figure 3. Two-step airframe shielding measurement procedure used in the Bombardier Global 5000 evaluation. 
penetration $(P E)$, was computed from the deconvolution:

$$
P E=D C \cdot\left|\frac{S_{42}(\text { gated airframe })}{S_{42}(\text { gated reference })}\right|^{2},
$$

where $S_{42}$ (gated reference) is the antenna-to-antenna coupling with environmental effects gated out, and $S_{42}$ (gated airframe) is the airframe transmission with gating applied to minimize noise. Since the reference and airframe measurements are typically carried out at different distances, a distance correction, $(D C)$, was applied.

Transmission measurement through airframe

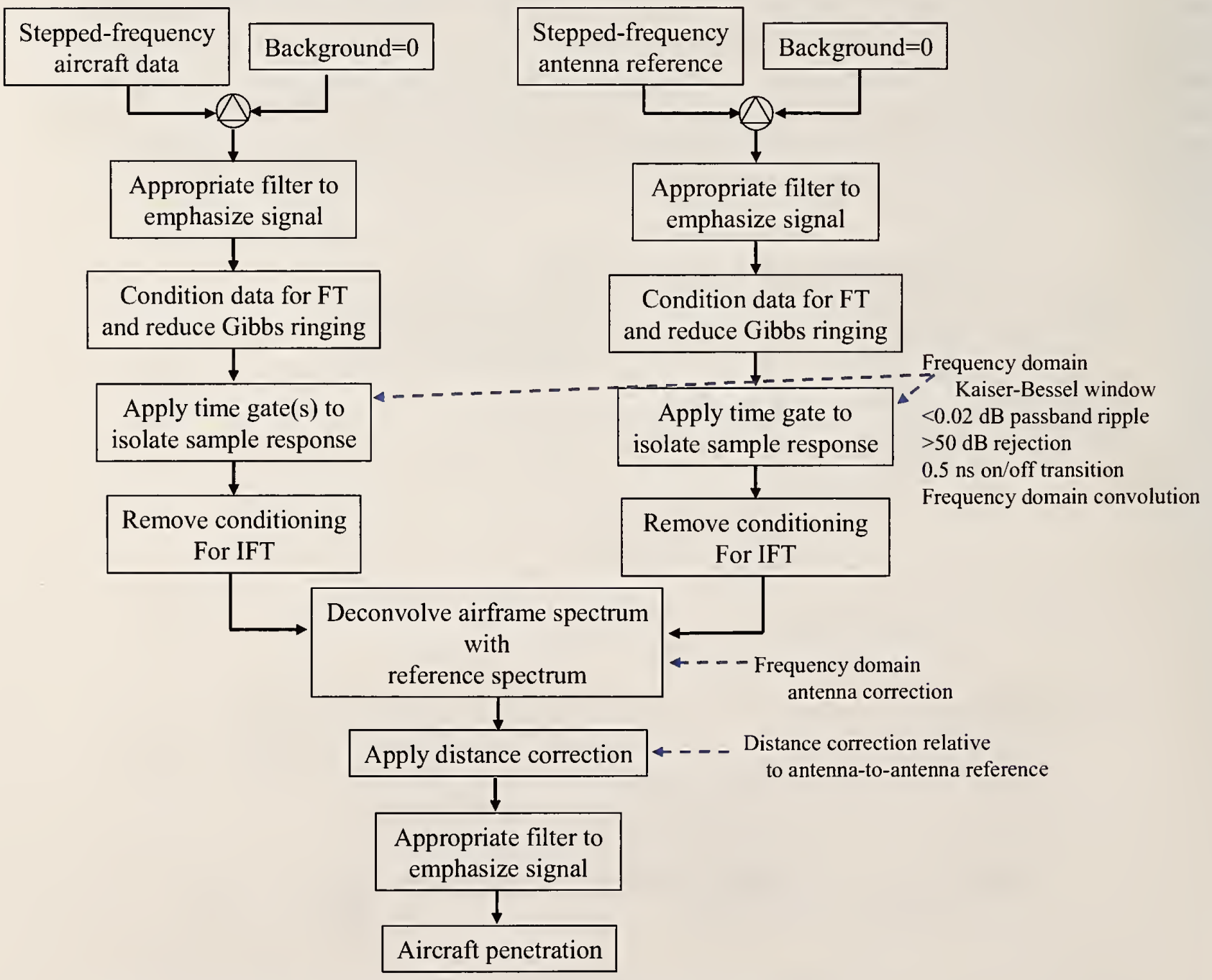

Figure 4. Signal processing sequence to obtain airframe penetration. 


$$
D C(d B)=20 \log _{10}\left(\frac{D}{D_{r e f}}\right),
$$

where $D$ is the distance in meters from the aperture of the transmitting antenna to the geometric center of the aircraft and $D_{\text {ref }}$ is the distance between the reference antennas. A detailed description of this procedure is given in [2]. Thus, our definition of penetration is based on a comparison of a direct antenna-to-antenna coupling and signal transmission through the airframe. The penetration, as we define it here, is the reciprocal of shielding (in decibels, penetration is the negative of shielding). We prefer to display results in terms of penetration because of a better graphical display and to normalize by our reference measurement.

Single aspect penetration characteristics for aircraft exhibit rapid variations with frequency, due to the large, complex cavity behavior of the aircraft. In order to obtain the volumetric averaged penetration for a compartment, the processed penetration data were frequency averaged over a specified bandwidth, by use of the process shown in Figure 5. Averaging the penetration data reduces the complex cavity variations, increases modal randomness, and highlights systematic coupling effects. These effects are discussed in detail in references [2-5].

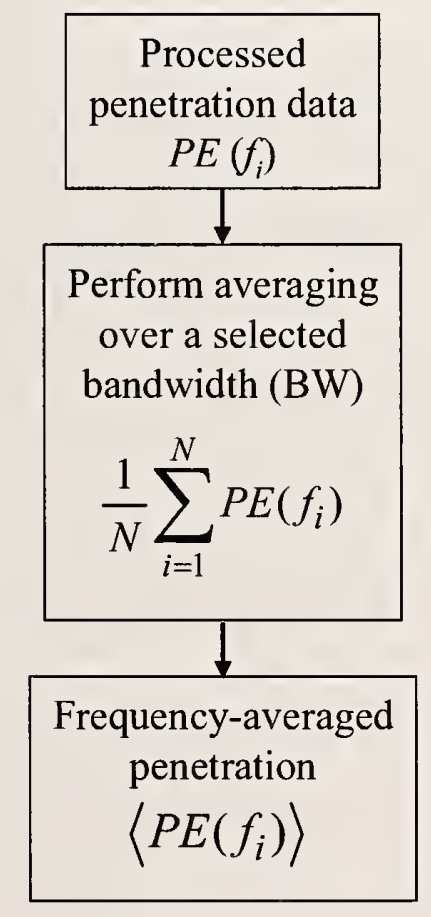

Figure 5. The frequency averaging definition. 


\section{Reference Measurements}

The test setup for the reference measurements is shown in Figure 6. The transmitting antennas are shown mounted on one tower with aperture centers located $4 \mathrm{~m}$ above the ground. The receiving antennas were mounted on the other tower. The towers were constructed from PVC pipes and plywood platforms to produce a low-density support structure with minimal scattering. The antennas were then boresighted and a distance extrapolation was performed to determine an optimal reference distance to minimize near-field effects and maximize the period between the signal and ground bounce. We performed multiple reference measurements with antenna-toantenna separations ranging from $2 \mathrm{~m}$ to $35 \mathrm{~m}$. We found that a separation of $6 \mathrm{~m}$ was optimal in terms of accuracy and time separation. Two transmission measurements were performed namely, one each for the vertical and horizontal channels. This process required a manual reorientation of the receiving antenna to be co-polarized with each of the transmitting antennas. Once the reference data were taken, the receiving antenna was dismounted from the support tower and placed at selected locations inside the aircraft. The external antenna tower and aircraft are shown in Figure 7. The reference waveforms for the DRG antenna are shown in Figures 8, 9, and 10. The reference waveforms for the TEM horn antenna are shown in Figures 11, 12, and 13. The ungated time-domain reference waveforms are shown in Figures 8 and 11. The doublets that occur at $18 \mathrm{~ns}$ in Figure 8 and $30 \mathrm{~ns}$ in Figure 11 correspond to the direct antenna-to-antenna coupling. The subsequent waveform activity was due to a combination of internal antenna

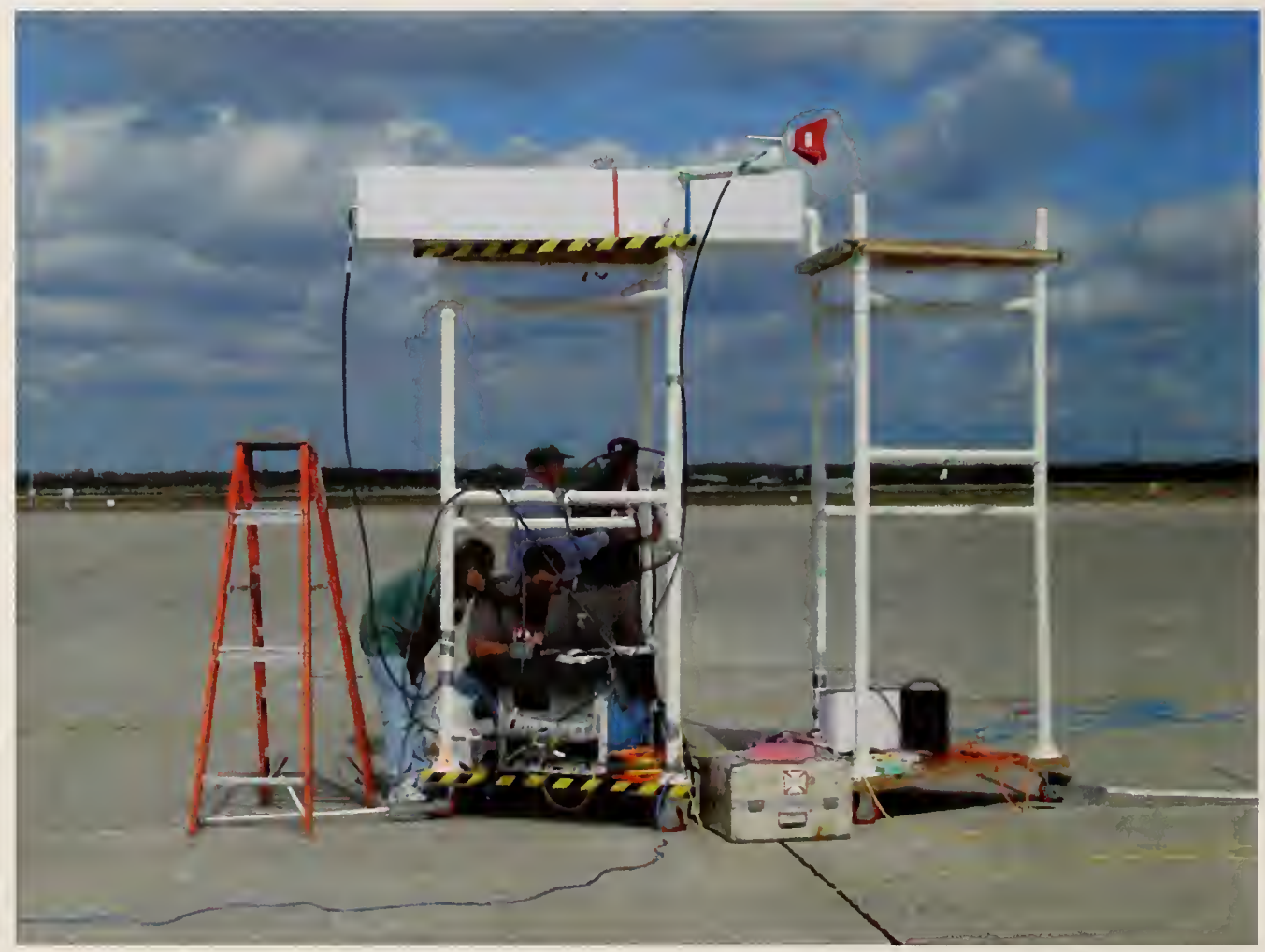

Figure 6. Reference measurement setup. The transmitting tower is configured and the receiving antennas will be mounted on top of the second tower. 
reflections and environmental scattering due to ground bounce and reflections from nearby objects. Figures 9 and 12 show the ungated frequency-domain responses for the DRG and TEM horn references, respectively. The noise-like hash on the waveform is due to ground bounce and spurious reflections which were time-gated out of the reference measurement. The frequencydomain transmission amplitude spectra of Figures 10 and 13 show the impact of applying this time gate, thus isolating direct antenna to antenna coupling and yielding the reference for penetration measurements.

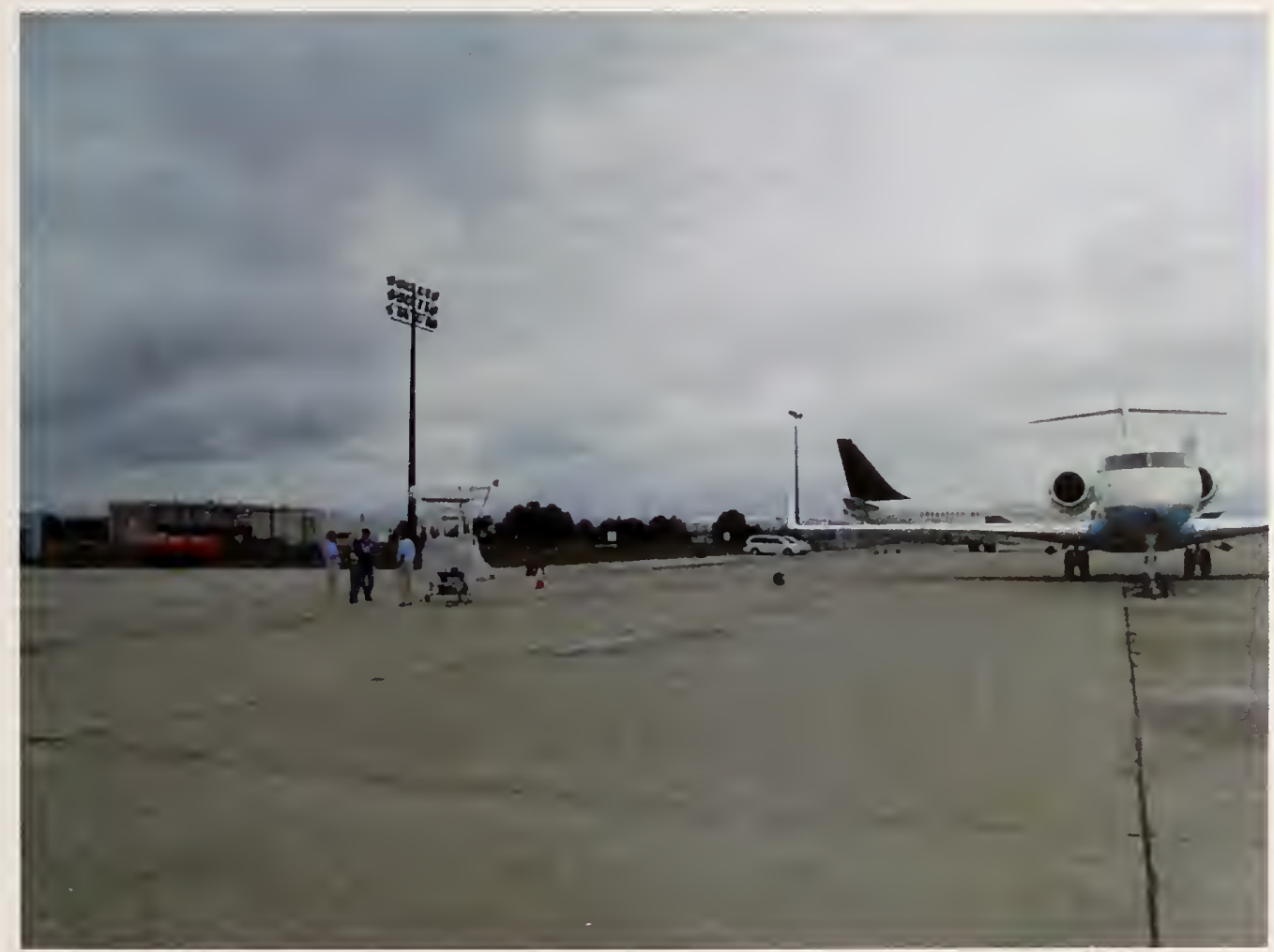

Figure 7. The transmitting antennas placed on top of a support tower and boresighted at the center of the aircraft. 


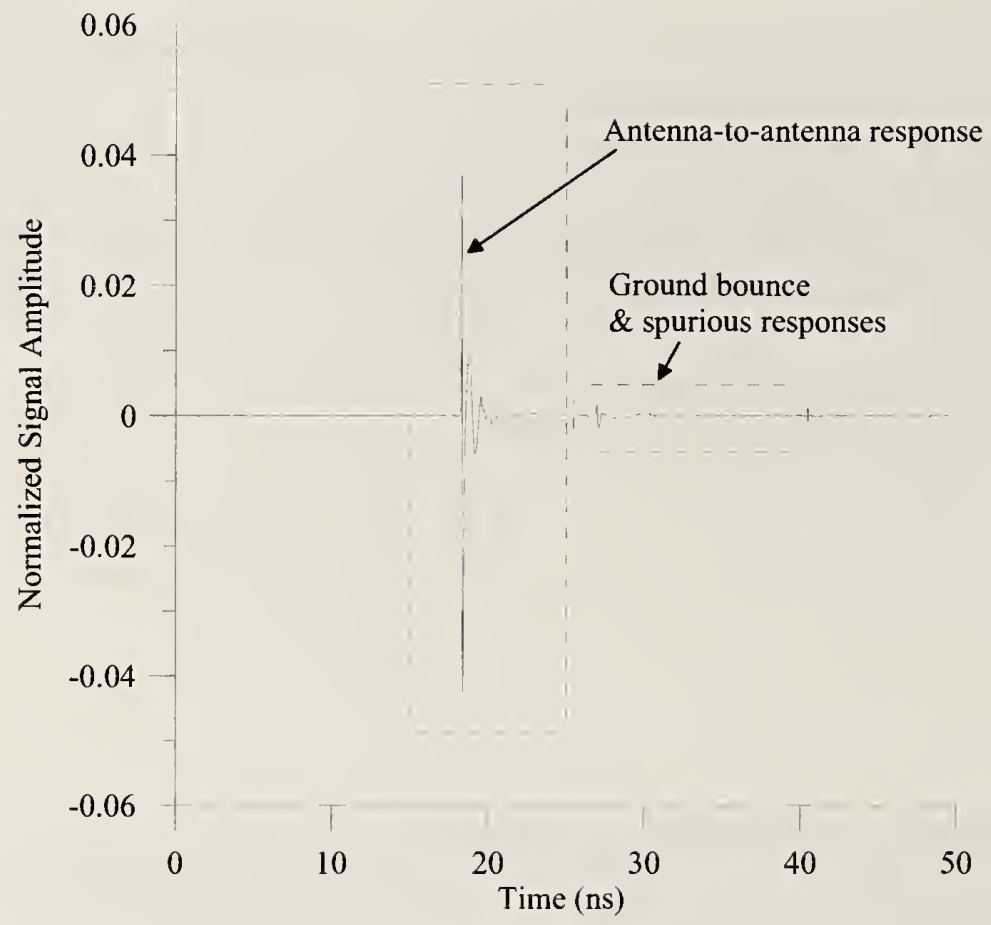

Figure 8. Ungated time-domain transmission waveform for a DRG antenna obtained at a separation of $6 \mathrm{~m}$ and vertical polarization.

0

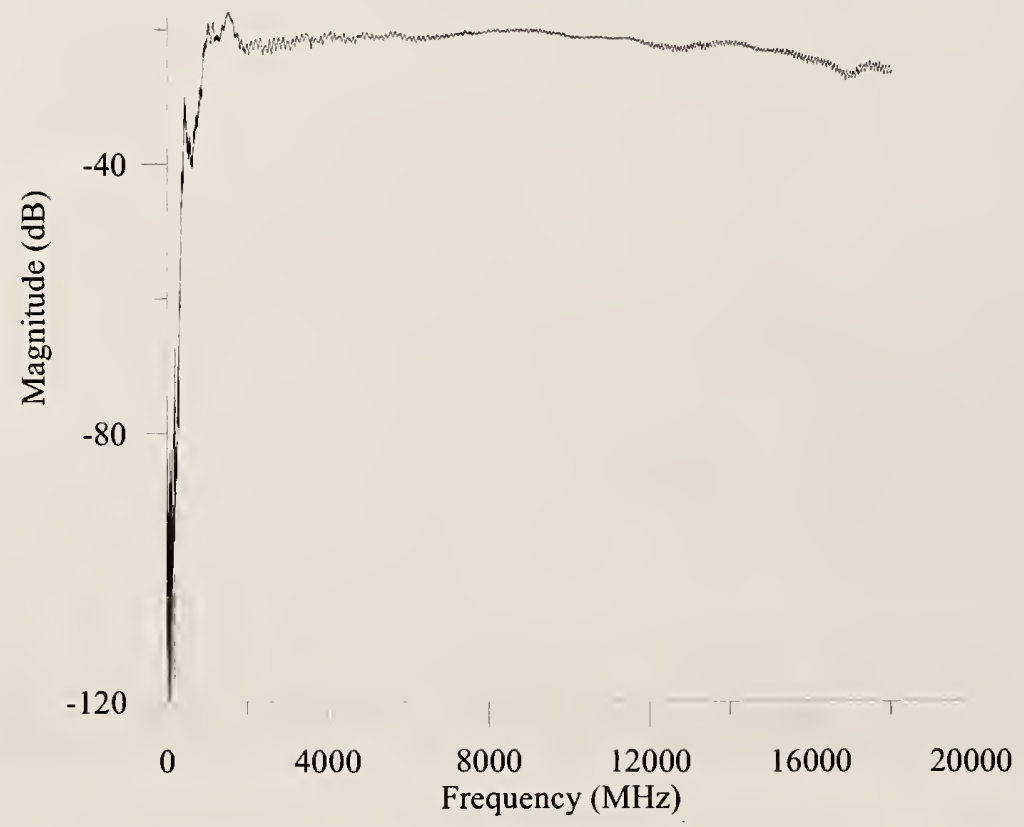

Figure 9. Ungated frequency-domain amplitude spectrum for a DRG horn antenna obtained at a separation of $6 \mathrm{~m}$ and vertical polarization. Note the hashy behavior due to the spurious reflections shown in Figure 8. 


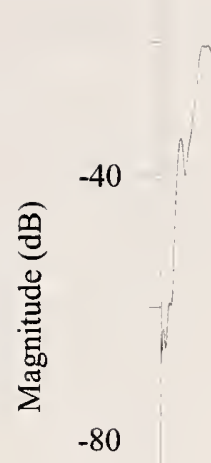

$-120$

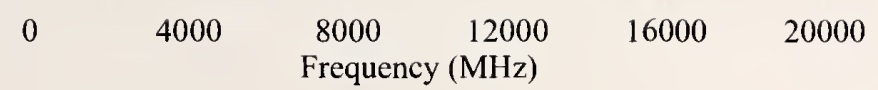

Figure 10. Gated frequency-domain amplitude spectrum corresponding to the waveform of Figure 9.

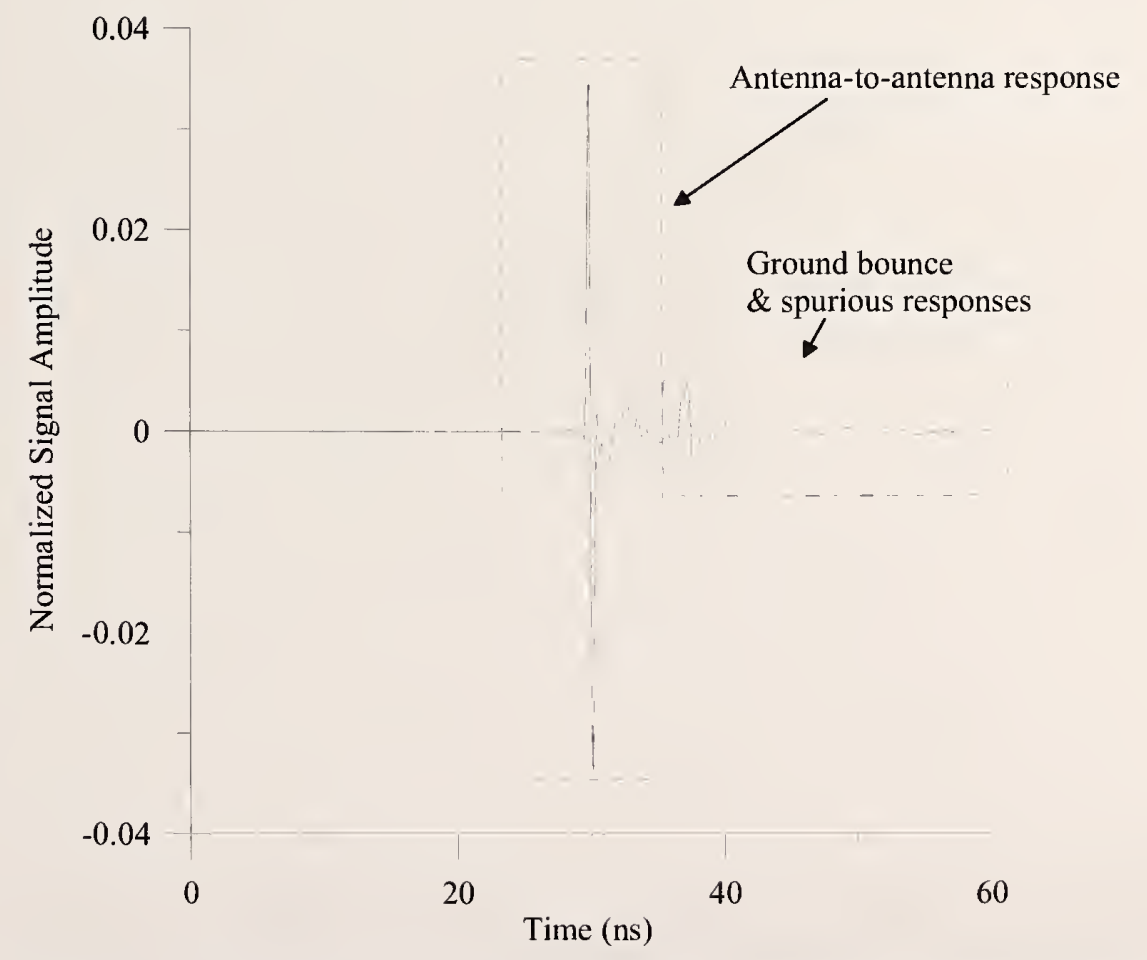

Figure 11. Ungated time-domain transmission waveform for a TEM antenna obtained at a separation of $6.0 \mathrm{~m}$ and vertical polarization. 


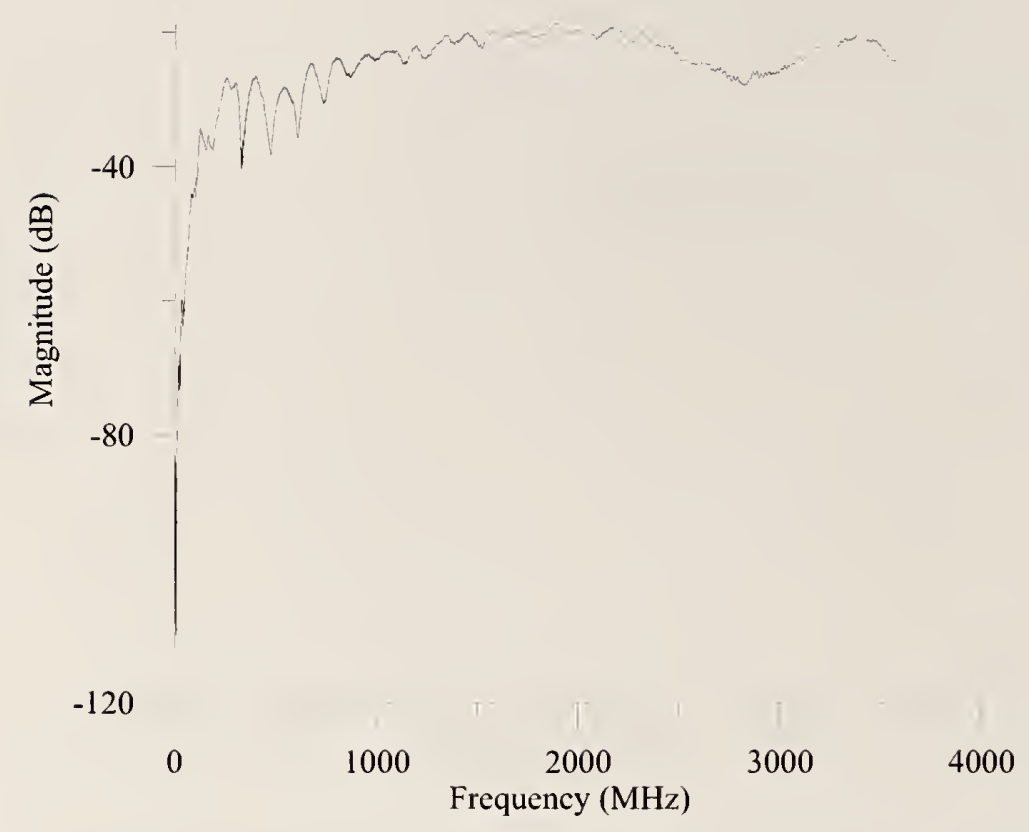

Figure 12. Ungated frequency-domain amplitude spectrum for a TEM horn antenna obtained at a separation of $6.0 \mathrm{~m}$ and vertical polarization. Note the scalloping due to the large ground bounce reflection shown in Figure 11.

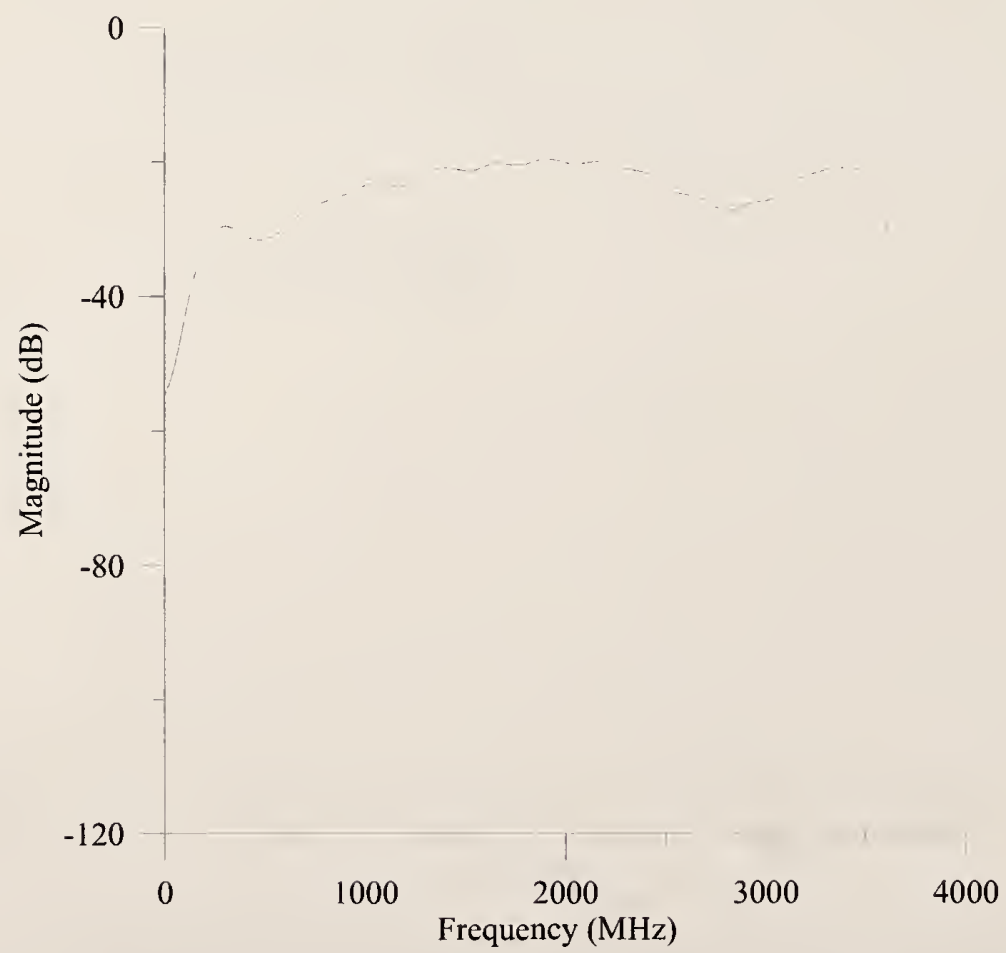

Figure 13. Gated frequency-domain amplitude spectrum corresponding to the waveform of Figure 12. 


\section{Airframe Penetration Measurements-Overview}

We began by measuring the penetration at several extrapolation points on the $45^{\circ}$ line as shown by the black X's in Figure 14. For penetration measurements, the external transmitting antennas were boresighted at the geometric center of the aircraft and moved to thirty clock positions at a radial distance of $23 \mathrm{~m}(75 \mathrm{ft})$ as shown by the blue, dashed line in Figure 14. We applied antenna gain and distance correction factors to maintain consistency in the results. The receiving antenna was placed in three compartments of the aircraft: (1) the flight deck, (2) the main cabin, and (3) the aft cargo closet. These are shown by the red X's.

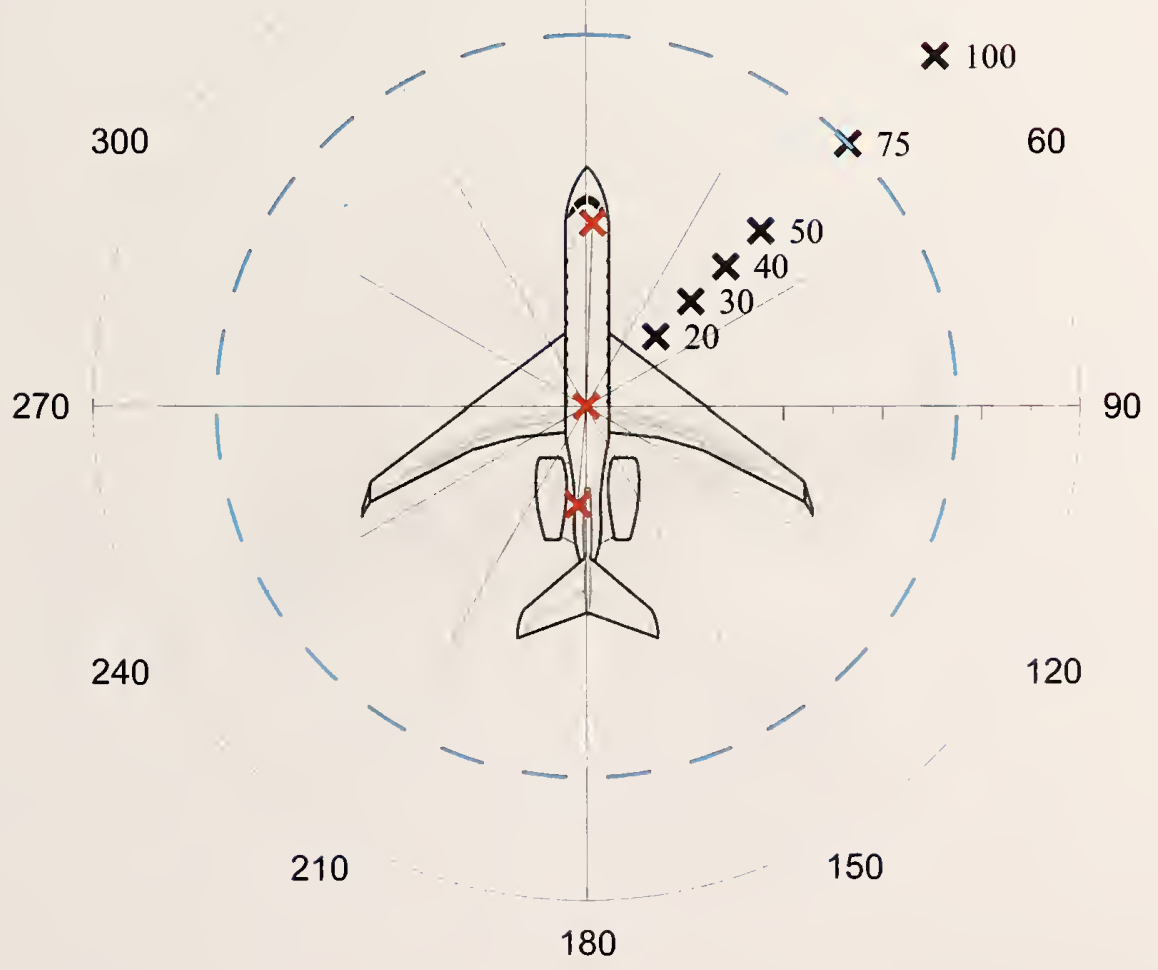

Figure 14. Test master graphic showing internal measurement positions (red X's), Extrapolation distances in feet (black X's), measurements around aircraft (blue, dashed line) at a distance of $23 \mathrm{~m}$ (75 ft). 


\subsection{Extrapolation Results}

We performed a set of extrapolation measurements from $6.1 \mathrm{~m}(20 \mathrm{ft})$ to $30.5 \mathrm{~m}(100 \mathrm{ft})$. The transmitting antennas were moved along the $45^{\circ}$ line and the receiving antennas were in the main cabin. These measurements were used to verify that we were in the far-field and to understand how penetration changes as a function of distance from the aircraft. Distance correction was applied to the data by use of a reference distance of $6.1 \mathrm{~m}$. These data are shown in Figures 15 to 18. These measurements show a spread in the data of about $5 \mathrm{~dB}$ to $6 \mathrm{~dB}$ across the frequency spectrum for the DRG antennas and around $10 \mathrm{~dB}$ for the TEM horn antennas. The large spread in the data is due mostly to the $6.1 \mathrm{~m}$ and $9.1 \mathrm{~m}$ distances. If we remove these data sets, we tighten up the spread to between $2 \mathrm{~dB}$ and $5 \mathrm{~dB}$ for both sets of antennas. We believe this is because, at the closer distances, our antennas see more of the aircraft, but as we move further from the aircraft the antennas see less of the aircraft and more of the surrounding environment. The DRG antennas have higher directivity than the TEM horn antennas, which corresponds to the greater spread in penetration data for the TEM horn antennas. These results have been both corrected for distance and for antenna gain. A discussion of the gain for the DRG horn antenna and TEM horn antenna is given in Appendix C.

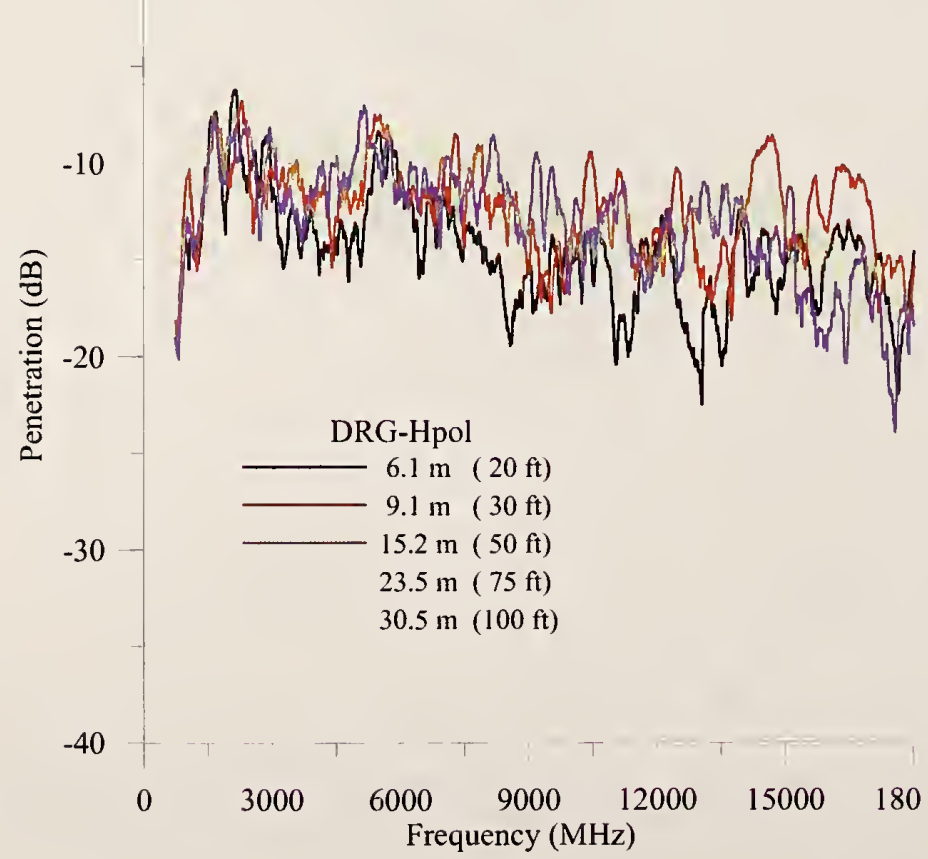

Figure 15. Extrapolation measurements for the DRG antenna, horizontal polarization. 


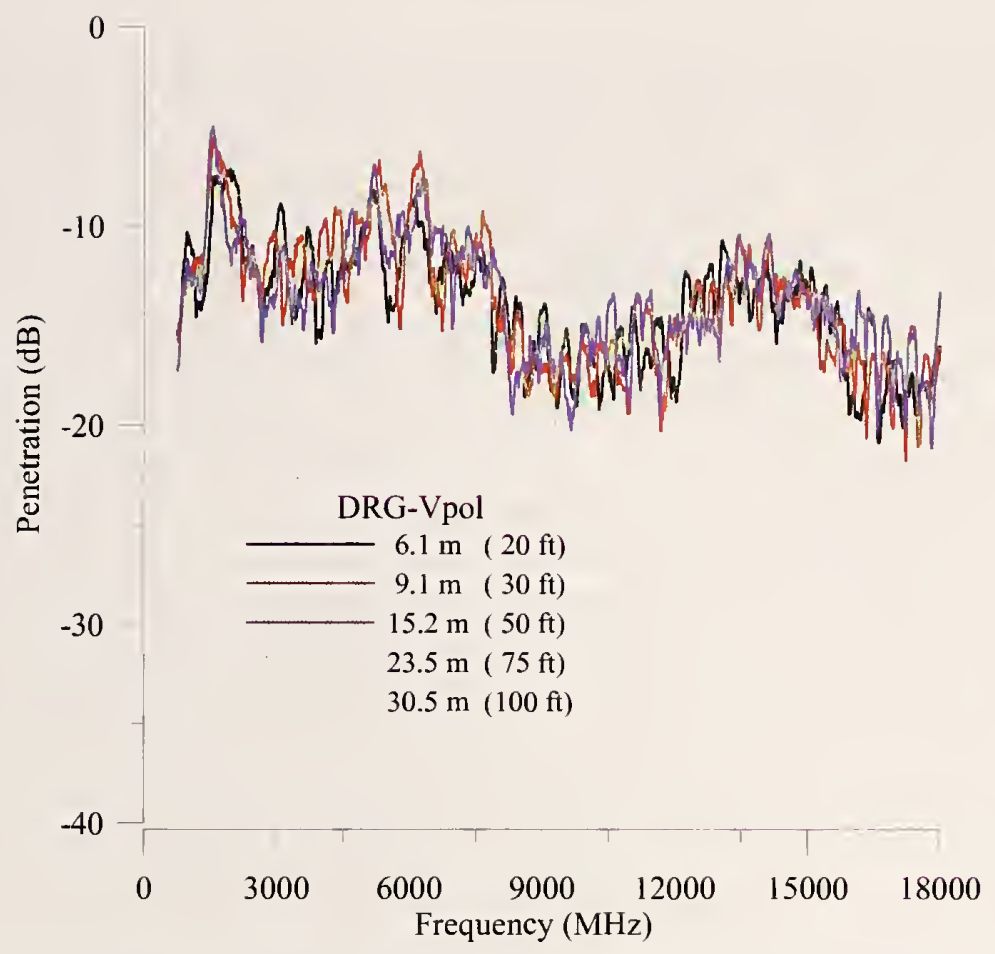

Figure 16. Extrapolation measurements for the DRG antenna, vertical polarization.

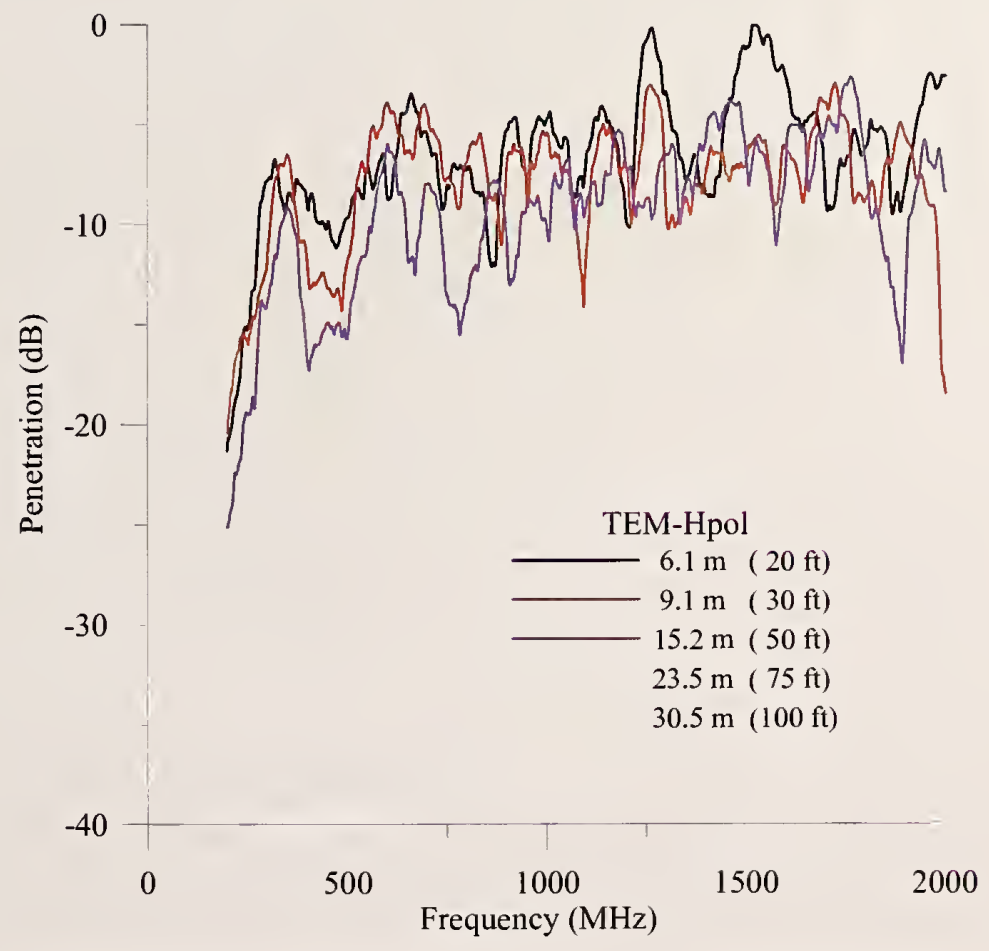

Figure 17. Extrapolation measurements for the TEM antenna, horizontal polarization. 


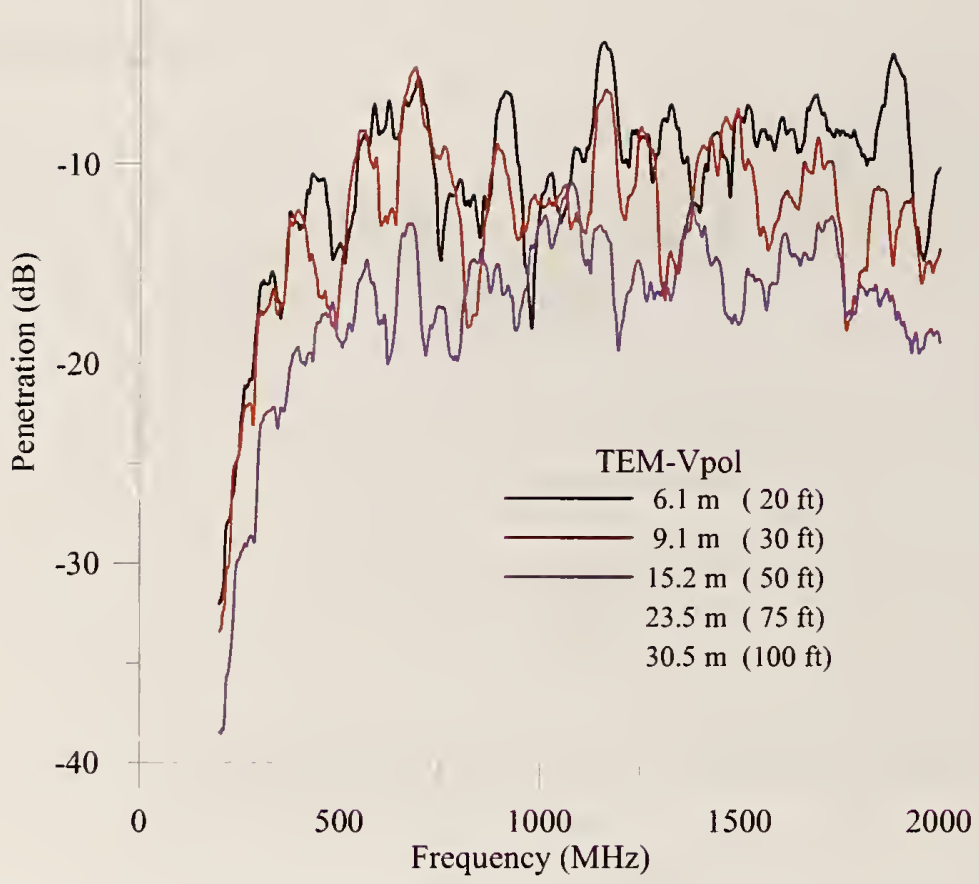

Figure 18. Extrapolation measurements for the TEM antenna, vertical polarization.

\subsection{Main Passenger Cabin Penetration Results}

The receiving antenna was located near the geometric center of the main cabin, as shown in Figure 19. The antennas were placed in an area where the seats had been removed and the antennas were mounted on tripods to provide support and separation. The antenna position was selected to provide robust excitation of available cavity modes. The transmitting antennas were boresighted to the geometric center of the aircraft to avoid assumptions about signal penetration apertures.

Time-domain plots are used as an aid to understand penetration into airframes. We begin by transforming the measured frequency domain data into the time domain to look at the signal in time. Time-domain plots for the DRG antenna in the horizontal polarization are shown in Figure 20 at various angular positions around the aircraft. At an angular position of $0^{\circ}$, we have small signal amplitude, where the signal rises to a maximum value and then decays slowly. This is indicative of reverberant field decay. As we travel around the aircraft we obtain maximum signals between $60^{\circ}$ and $150^{\circ}$. At these positions, the signal shows an initial sharp impulse, indicative of direct antenna-to-antenna coupling; it then falls and rises to a smaller amplitude and then decays again, showing the same reverberation characteristics as for the $0^{\circ}$ position. We know that the second smaller impulse is not due to ground bounce based on time-of-flight calculations. The cause of this second impulse is a build-up of fields inside the cavity followed by their decay. At angular positions between $170^{\circ}$ and $190^{\circ}$ we again observe small signal amplitudes. This makes sense because most of the signal will couple into the main cabin 
passenger windows, giving us the large initial direct coupling component seen at the beginning of the waveform. The small signal level at the back of the aircraft is because no direct signal path exists for the signal to get into the main passenger cabin, and at the front of the aircraft, the signal must travel through the flight deck windows and back into the main passenger cabin. The larger the direct coupling component, the more energy there is that initially gets coupled into the main passenger cabin, and the less energy there is available in the reverberant field. A more indepth discussion of this direct component versus reverberant field energy is found in Appendix A. This analysis provides valuable insight into possible maximum coupling paths. Time-domain plots for the DRG antenna in the vertical polarization are shown in Figure 21, and time-domain plots for the TEM horn antennas in both polarizations are shown in Figures 22 and 23. The TEM horn antennas receive lower signal levels and we do not see time-domain evidence of direct coupling. This could be due to the low directive gain (broad radiation pattern) of the antenna. Frequency-domain plots for various positions around the aircraft are summarized in Figure 24. The purpose of this plot is to show how the penetration level varies at selected positions around the aircraft. We expect the signal to drop at the front and back of the aircraft because there are no direct signal paths at these positions. We have also plotted some penetration results at various symmetrical positions around the aircraft to determine whether there may be leakage points on one side that are not on another. This is shown in Figure 25. We would expect a possible leakage point at the door into the aircraft. In Figure 24, we see that the maximum penetration of about $0 \mathrm{~dB}$ occurs around $12000 \mathrm{MHz}$ for the DRG antennas, with the transmitting antenna in

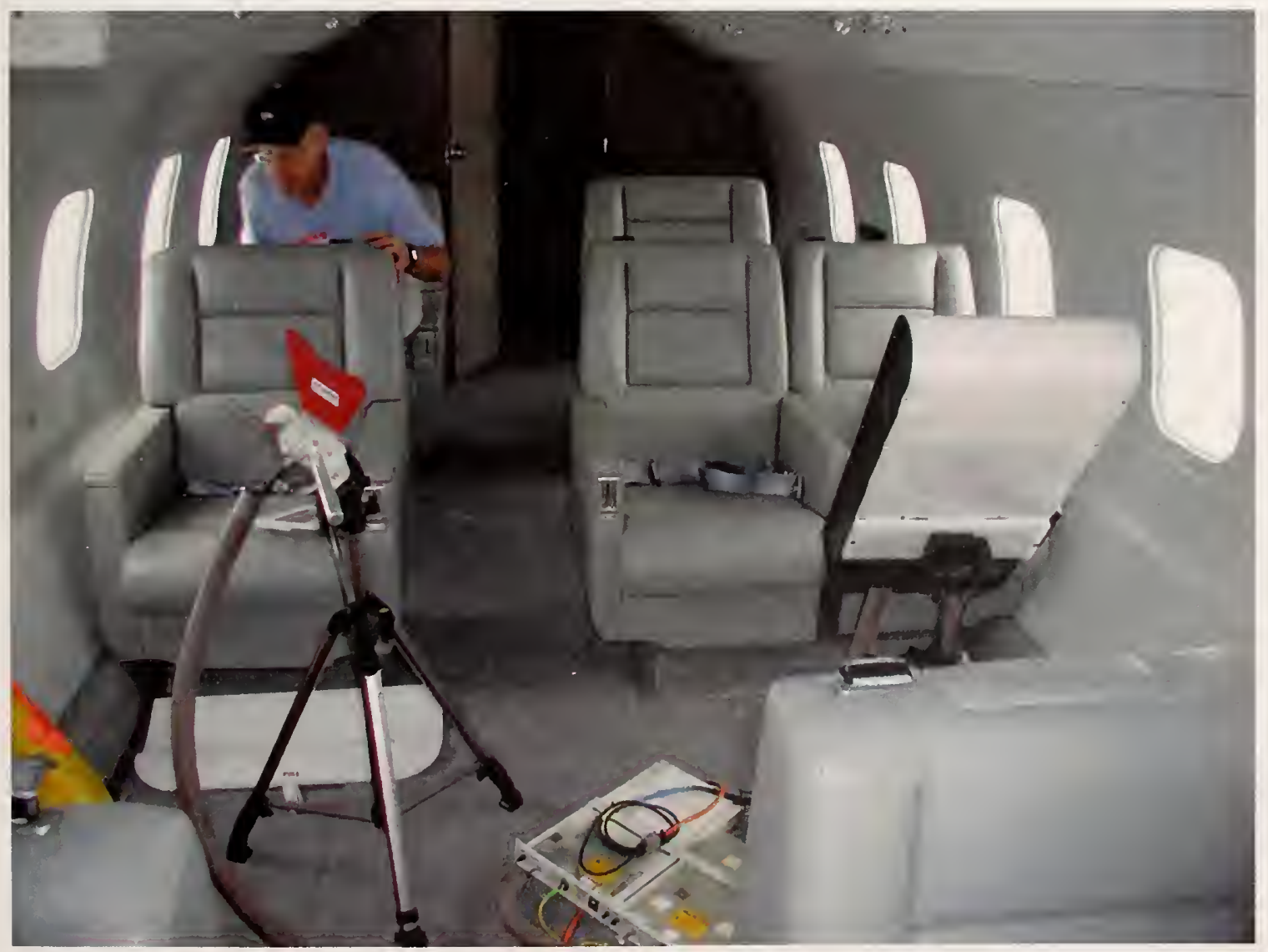

Figure 19. Receiving antenna placement in main passenger cabin. 
the vertical and horizontal polarizations, at an angle of $30^{\circ}$, and the receiving antennas in the main passenger cabin. Minimum penetration of approximately $-35 \mathrm{~dB}$ occurs at an angle of $180^{\circ}$ for the DRG antenna in the vertical polarization. Although not shown in either Figure 24 or Figure 25 , the signal is also strong in some of the $5^{\circ}$ positions; namely, $125^{\circ}$ and $145^{\circ}$. These positions are shown individually in Figures 26 to 53. At these particular positions there must be some direct path available to the signal. For TEM horn antenna signals below $100 \mathrm{MHz}$, we find that the penetration artificially increases, which is an artifact of the measurement system rapidly losing dynamic range. This reduction in dynamic range is due to the combination of increasing airframe shielding performance and reduced antenna efficiency.

Detailed penetration results at various azimuthal positions for all antennas and all polarizations are summarized in Figures 26 to 53. The DRG antenna data are frequency averaged over a bandwidth of $200 \mathrm{MHz}$ and the TEM horn antenna data are frequency averaged over a bandwidth of $50 \mathrm{MHz}$. We have concluded that these bandwidths work well at suppressing the "hashy" behavior caused by the complex cavity modes, while emphasizing the deterministic coupling effects. Frequency averaging is described in detail in [2-5]. We see that the penetration is lower at the $0^{\circ}$ position than for surrounding positions and increases as we proceed to the side of the aircraft. The highest penetration values are seen for positions from approximately $90^{\circ}$ to $150^{\circ}$. This directly correlates to the time domain data discussed previously. The horizontally polarized antennas tend to have higher penetration values than the vertically polarized antennas and the vertically polarized DRG antenna has very low penetration values from approximately $330^{\circ}$ to $350^{\circ}$. Our purpose in moving every $10^{\circ}$ until we reach the second quadrant and then changing to $5^{\circ}$ increments is to investigate the possibility that we may be missing important leakage points by only traversing every $10^{\circ}$ [4]. What we see is that the angular variation in penetration does not change greatly from angular position to position with typical variations less than $5 \mathrm{~dB}$. This can be attributed to a combination of complex cavity behavior and a large number of coupling apertures distributed around the perimeter of the main cabin. Due to the large number of coupling apertures, we do not take into account the antenna pattern effects for either the antennas or the aircraft. If we knew exactly where the fields were penetrating into the cavity, then these types of corrections would be feasible. These coupling paths are both vertically and horizontally polarized, and therefore we believe the fields inside the aircraft are depolarized to a large extent.

The main conclusions for an antenna placed in the main passenger cabin are: (1) the main coupling apertures are the windows on the side of the aircraft; (2) penetration is lowest at the front and back of the aircraft due to lack of coupling apertures; (3) penetration into the airframe is fairly symmetric except where the door to the aircraft is; (4) typical penetration values are 0 $\mathrm{dB}$ from $90^{\circ}$ to $140^{\circ}$ and $-30 \mathrm{~dB}$ for the $0^{\circ}$ and $180^{\circ}$ positions; and (5) horizontally polarized energy more easily penetrates the airframe than vertically polarized energy. We believe this is because of the horizontal "slot apertures" that the array of windows provides. 


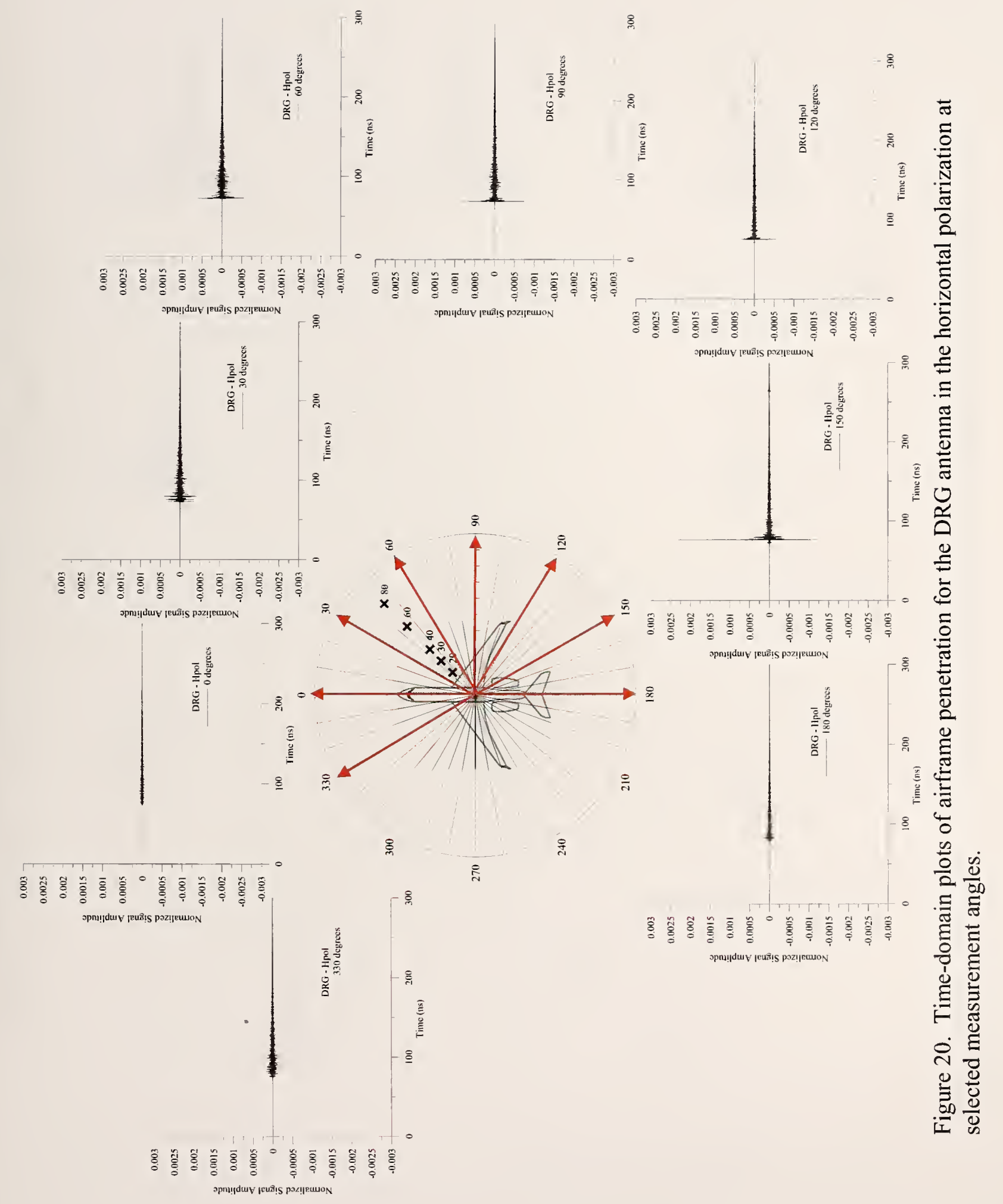




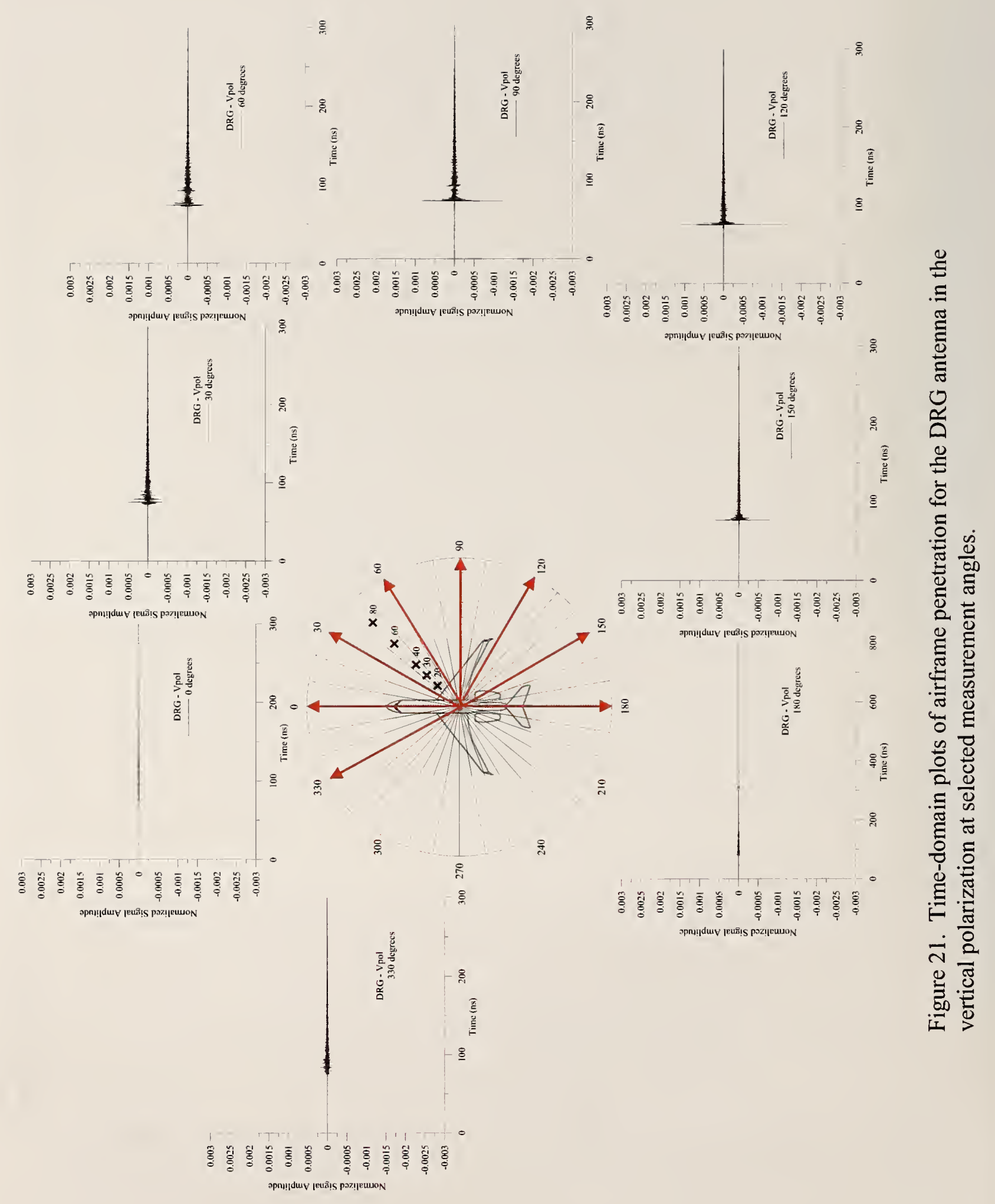




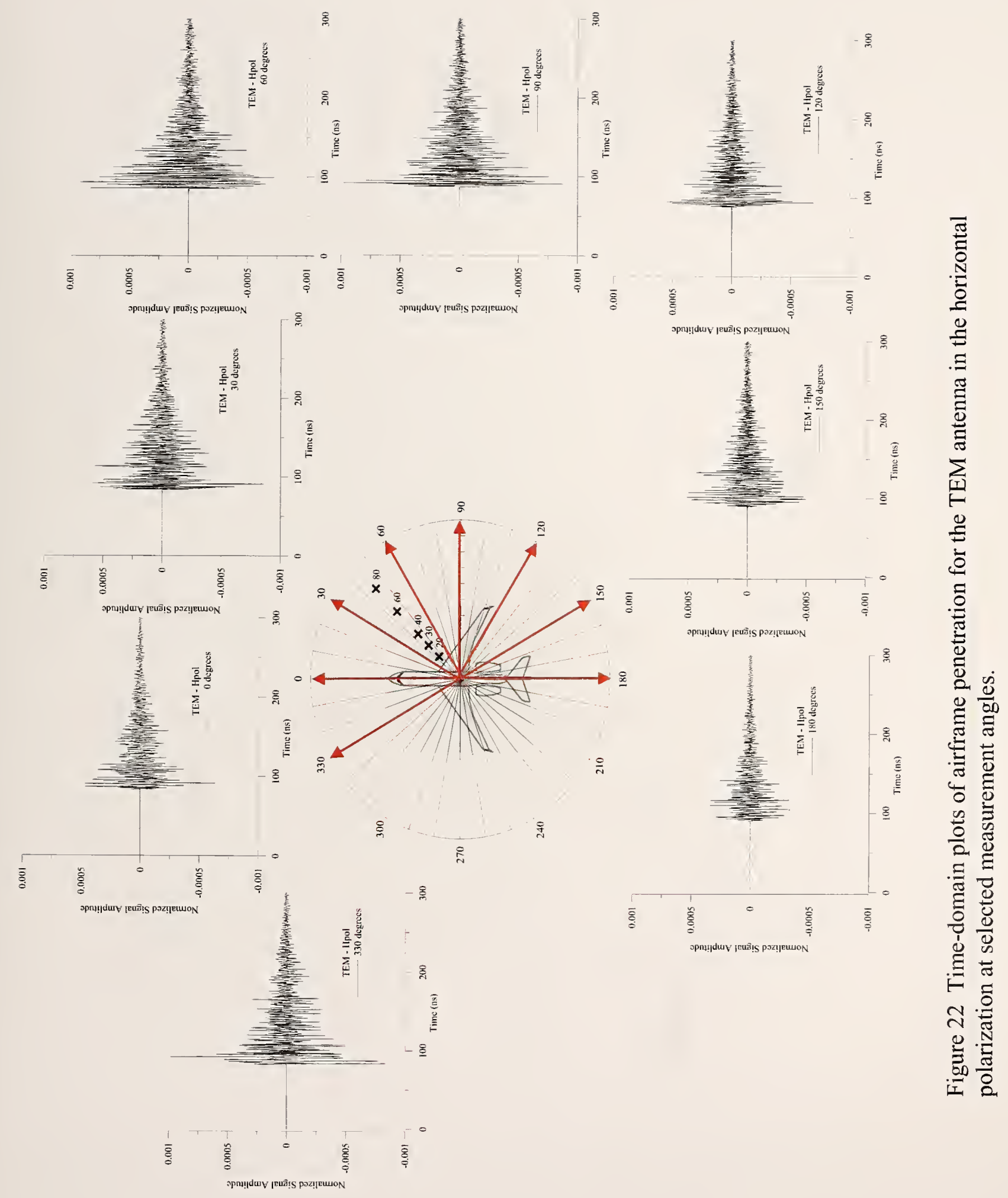



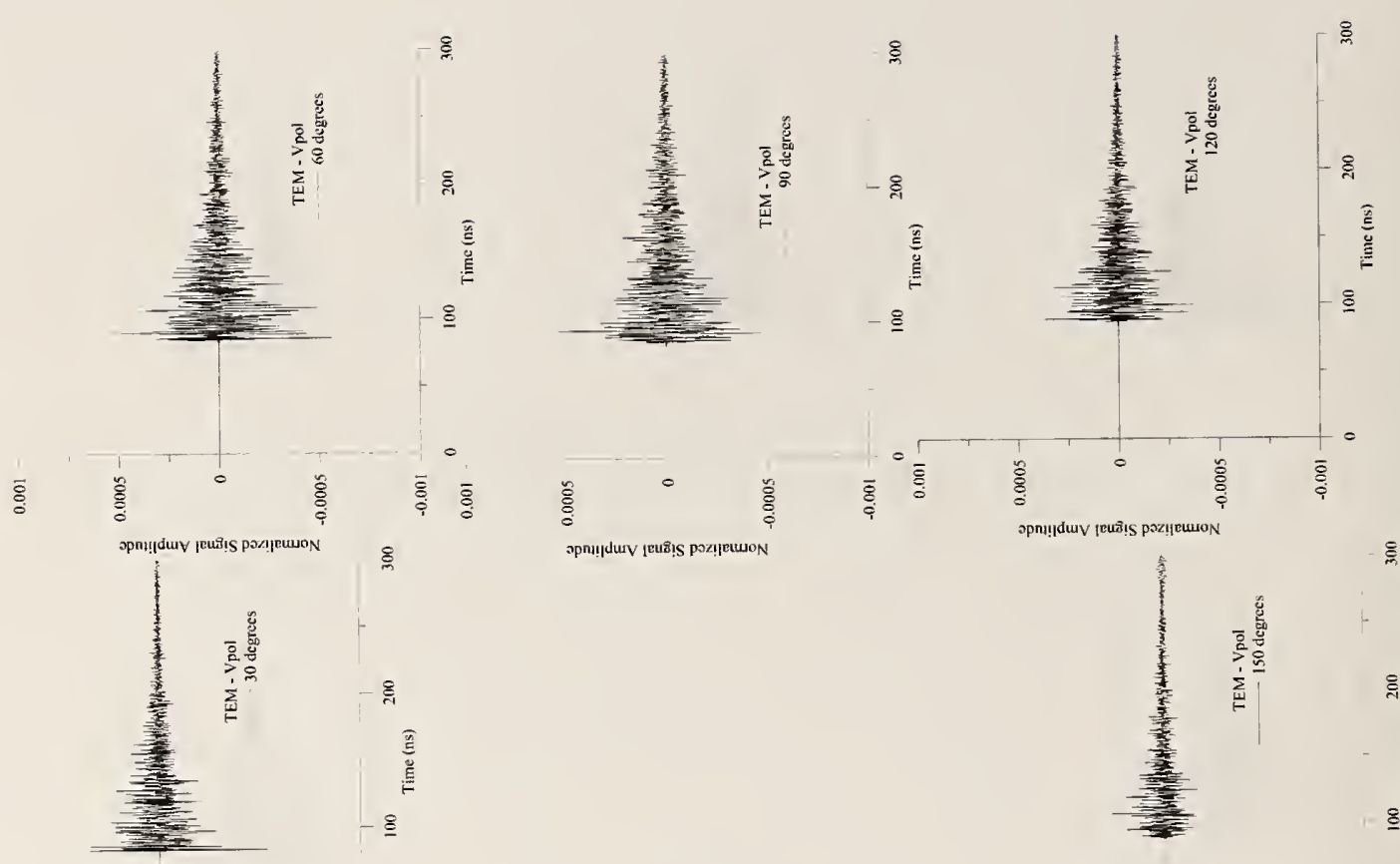

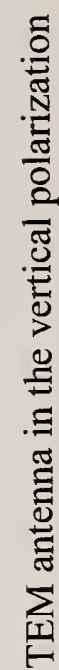

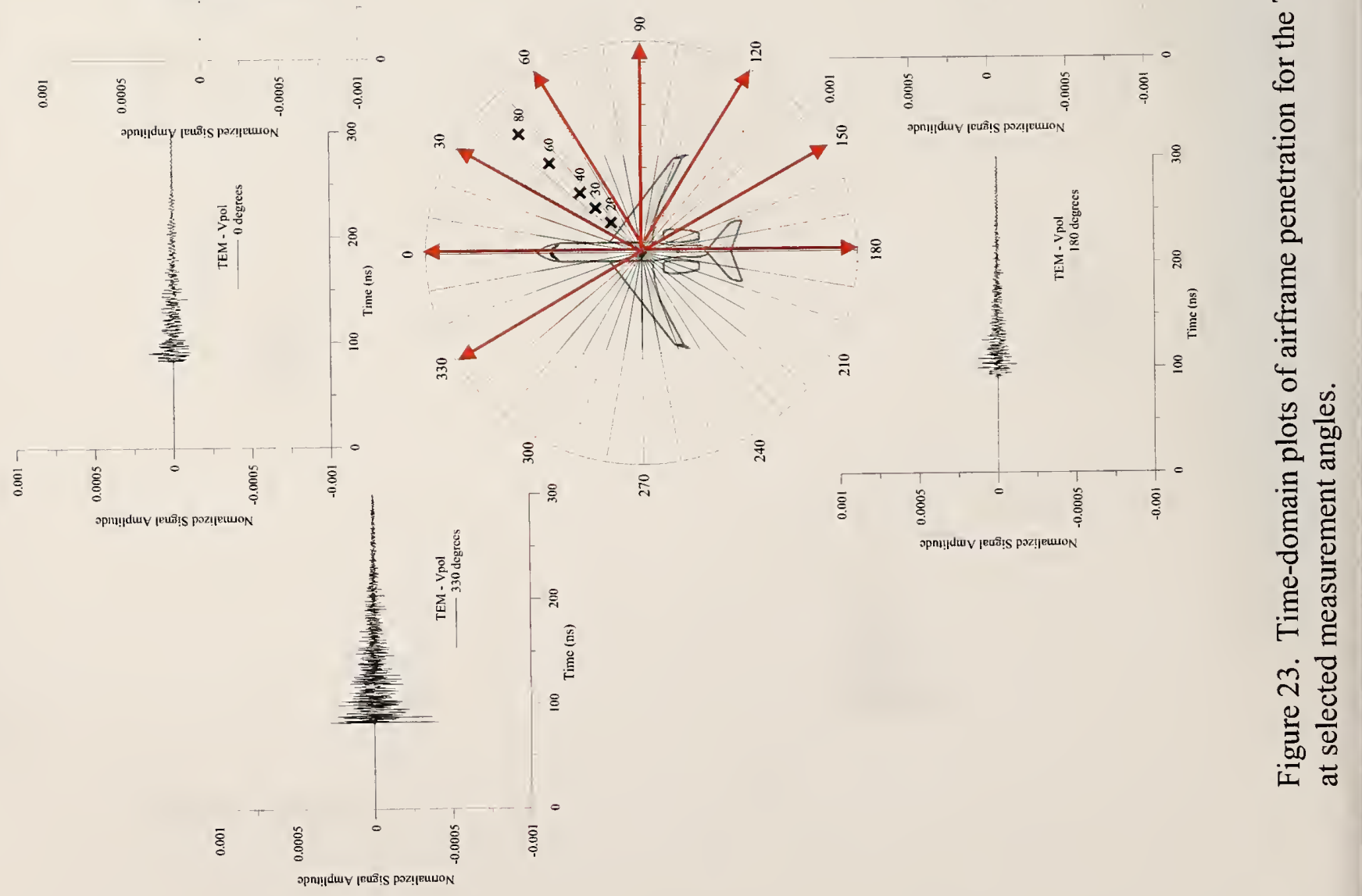




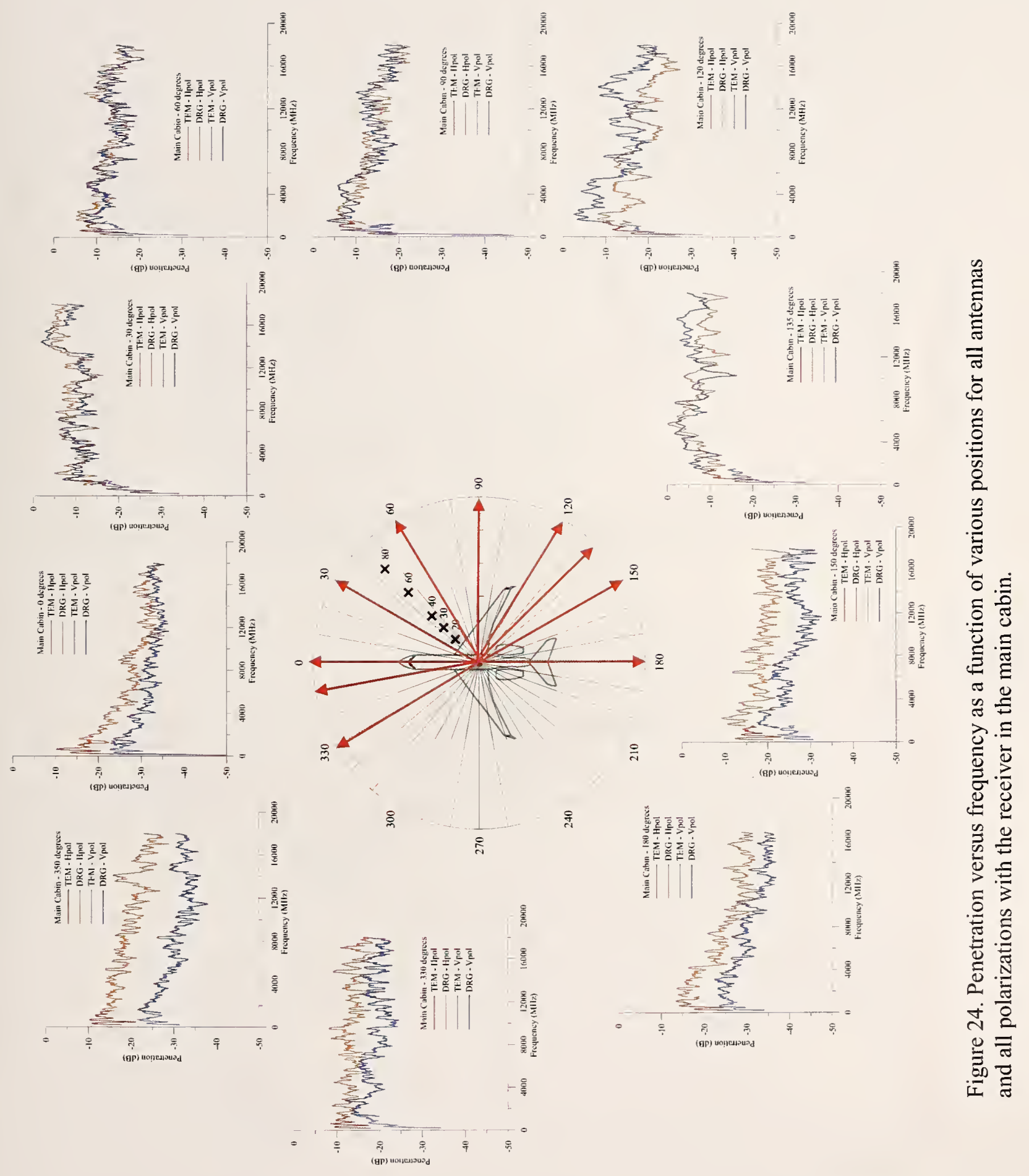




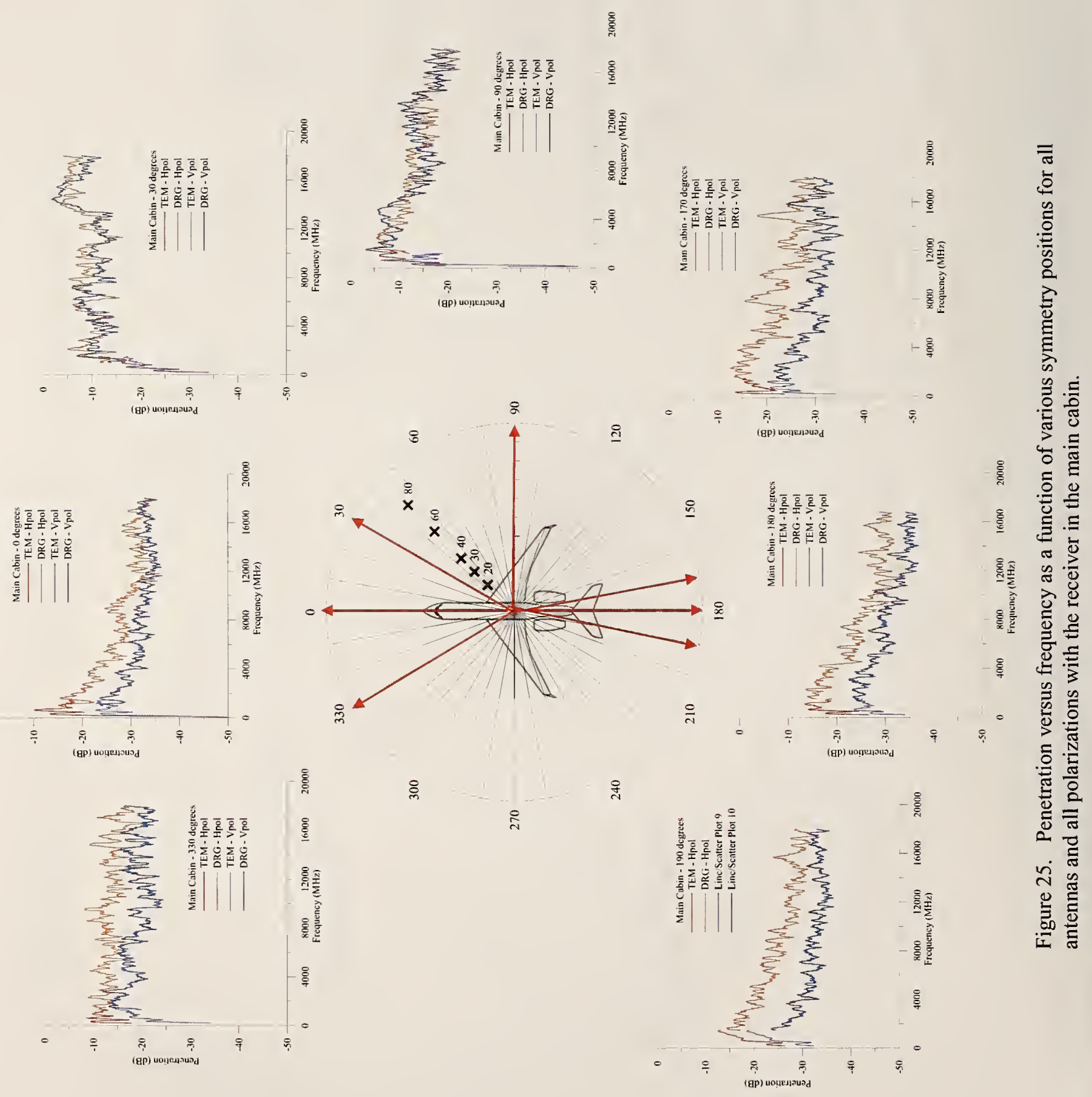




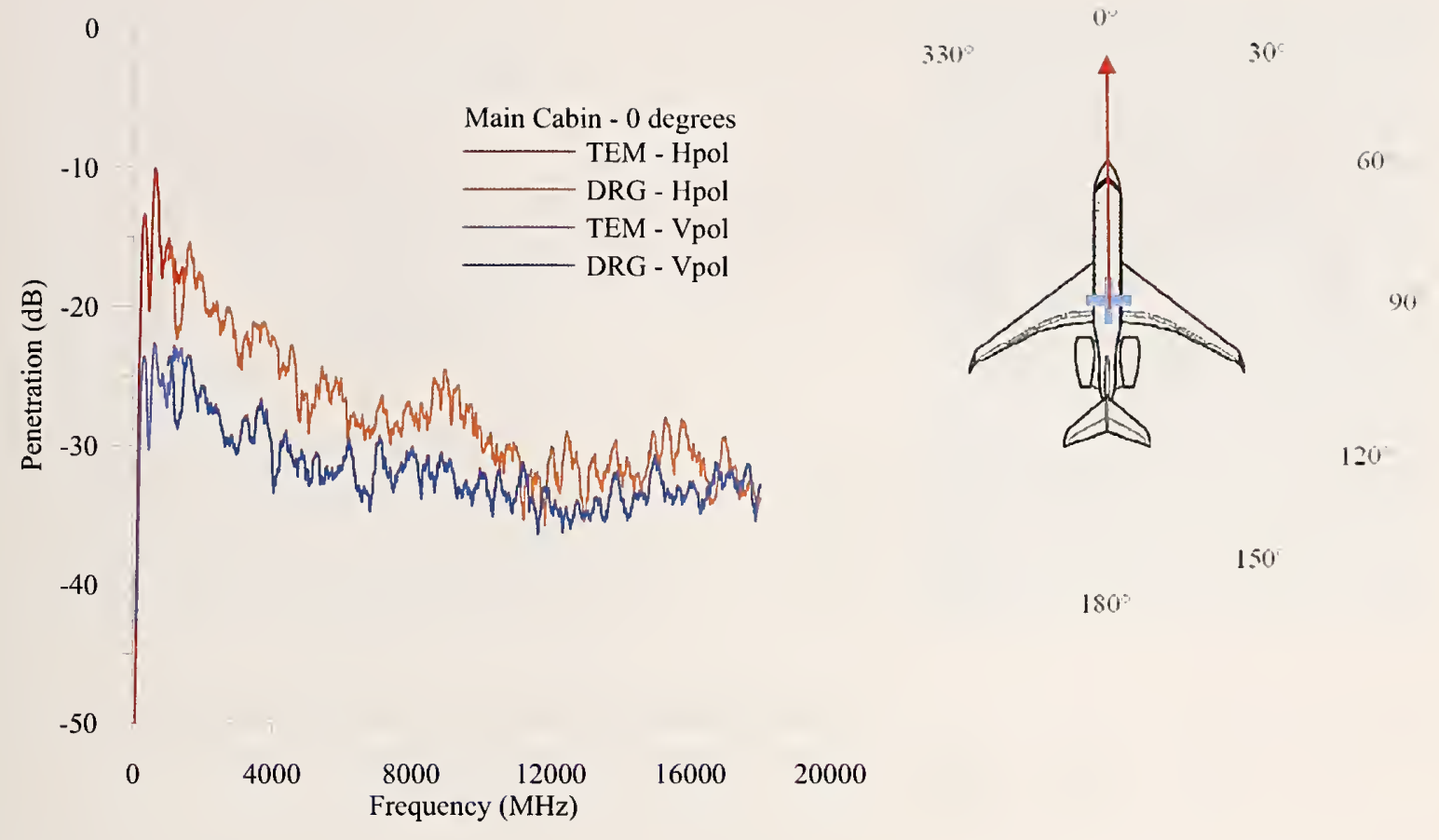

Figure 26. Penetration versus frequency for all antennas and polarizations for the transmitting tower placed at the $0^{\circ}$ position and the receiver placed in the main cabin.
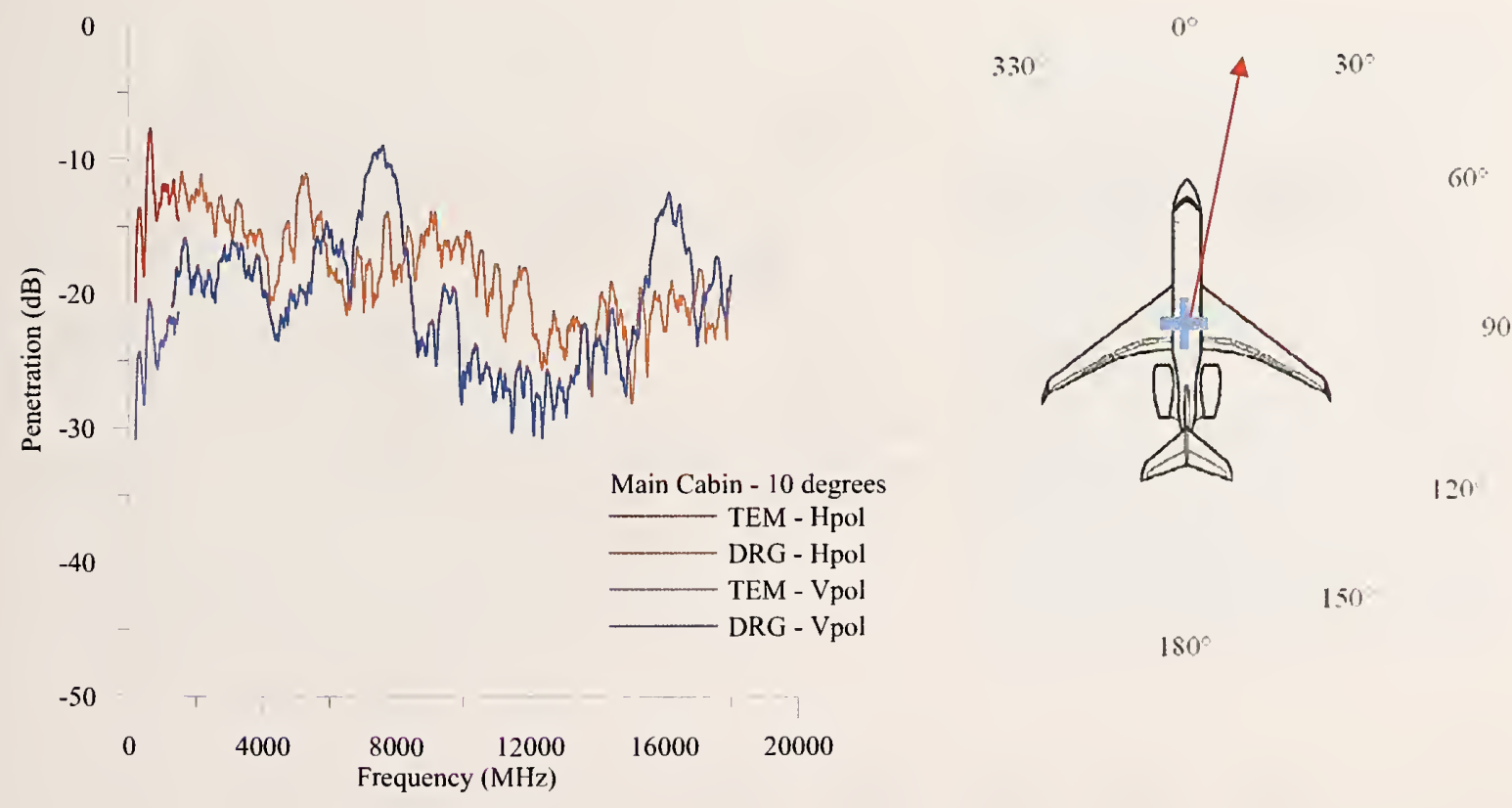

Figure 27. Penetration versus frequency for all antennas and polarizations for the transmitting tower placed at the $10^{\circ}$ position and the receiver placed in the main cabin. 

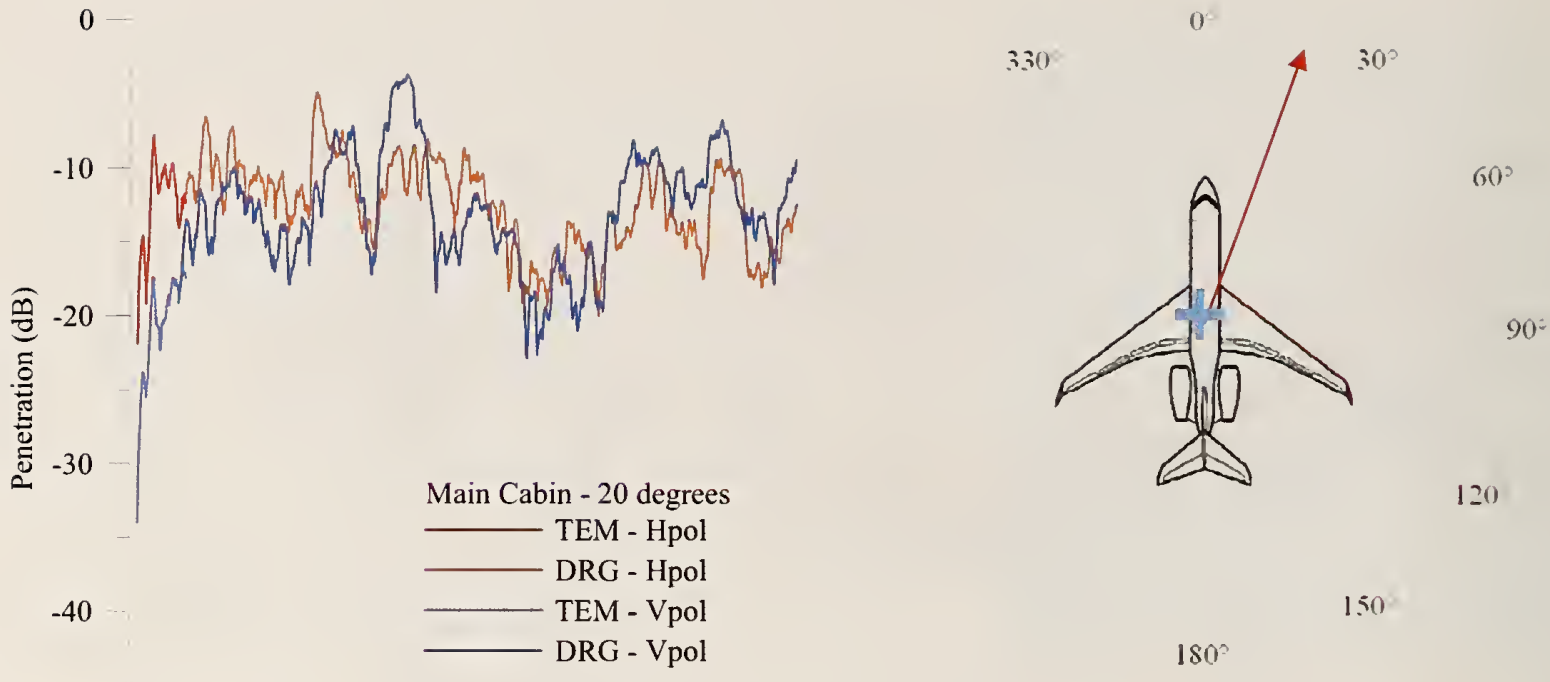

$-50$

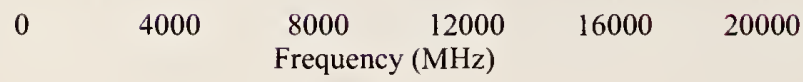

Figure 28. Penetration versus frequency for all antennas and polarizations for the transmitting tower placed at the $20^{\circ}$ position and the receiver placed in the main cabin.
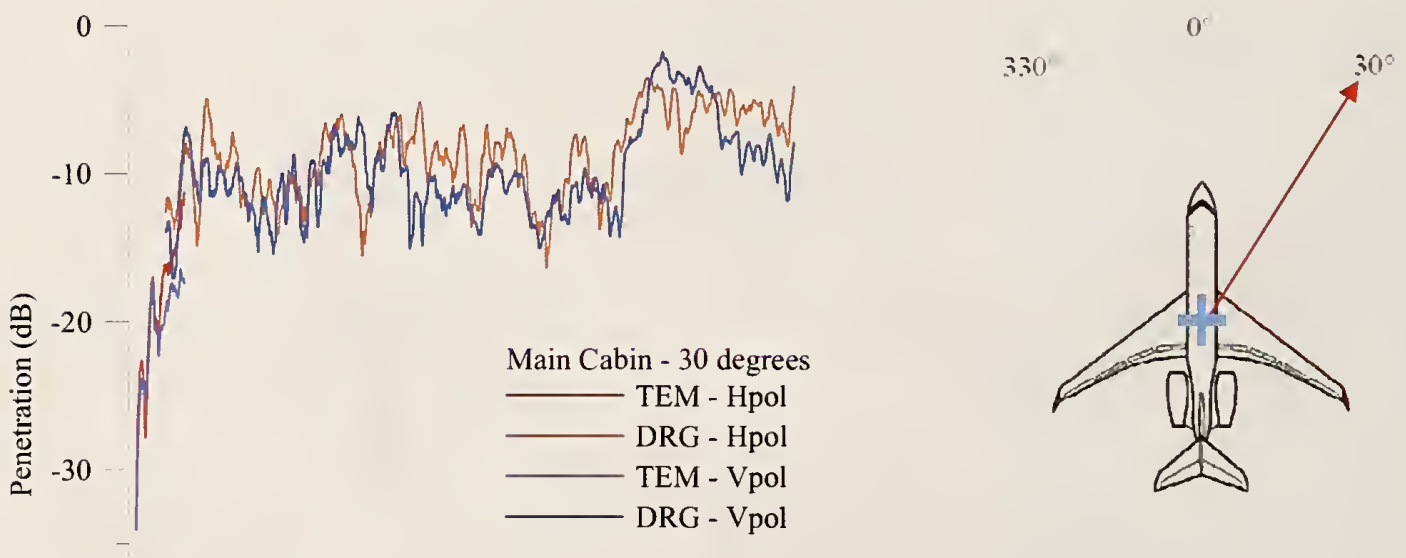

$60^{\circ}$

$90^{\circ}$

$-40$

150

$180^{\circ}$

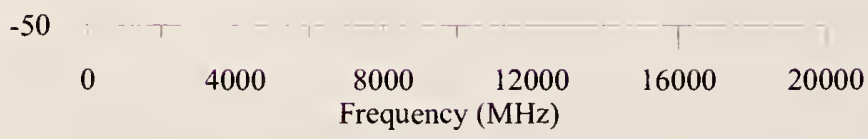

Figure 29. Penetration versus frequency for all antennas and polarizations for the transmitting tower placed at the $30^{\circ}$ position and the receiver placed in the main cabin. 
0

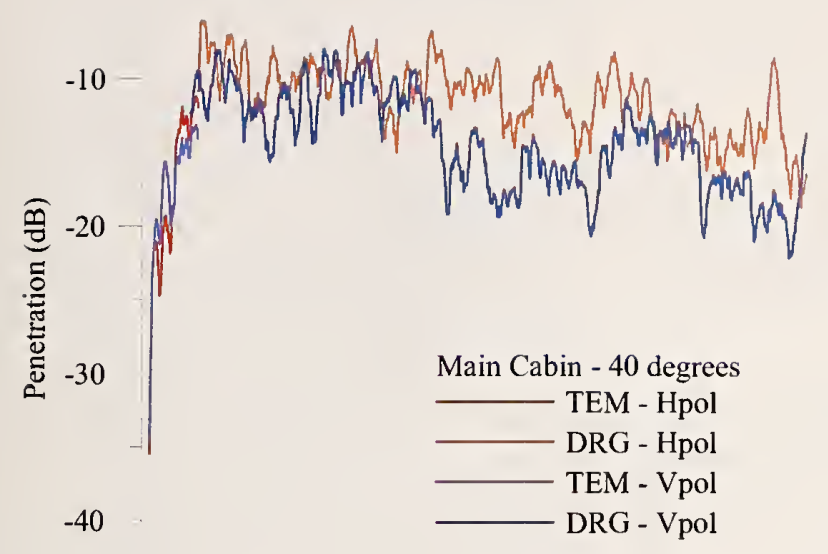

3.30

()

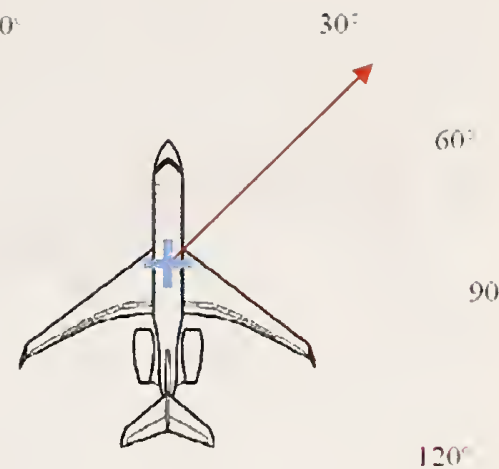

150

$180^{\circ}$

$-50$

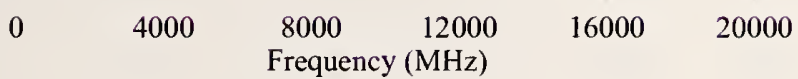

Figure 30. Penetration versus frequency for all antennas and polarizations for the transmitting tower placed at the $40^{\circ}$ position and the receiver placed in the main cabin.

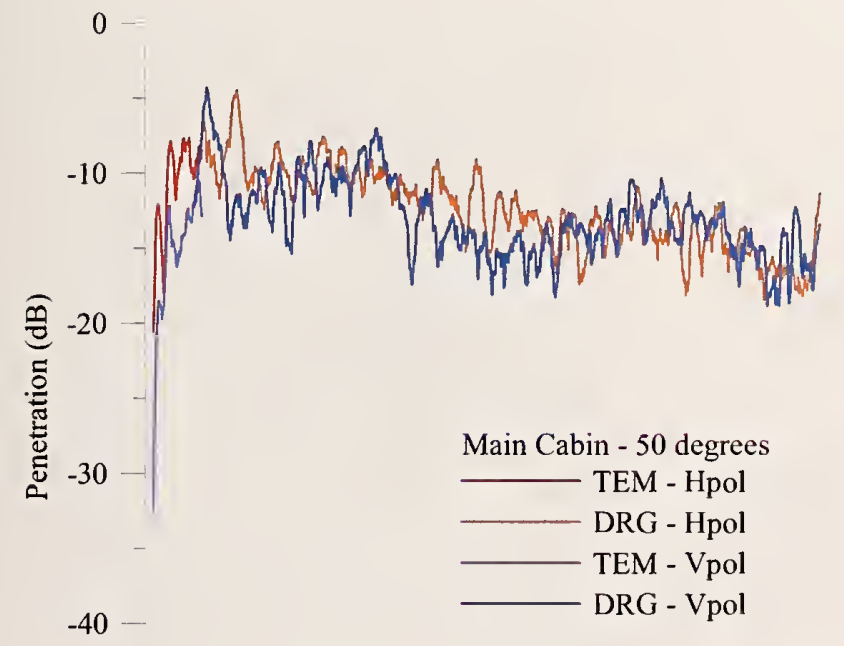

3.30 $0^{\circ}$

30

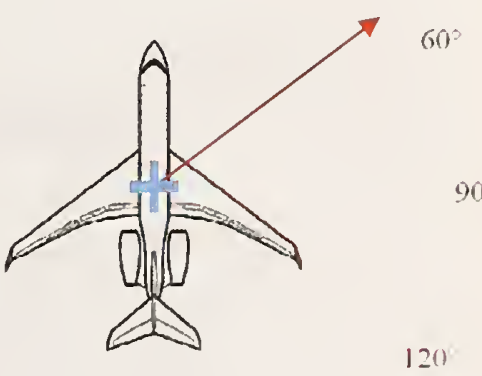

150

180

$-50$

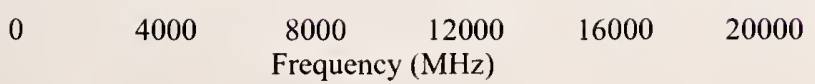

Figure 31. Penetration versus frequency for all antennas and polarizations for the transmitting tower placed at the $50^{\circ}$ position and the receiver placed in the main cabin. 


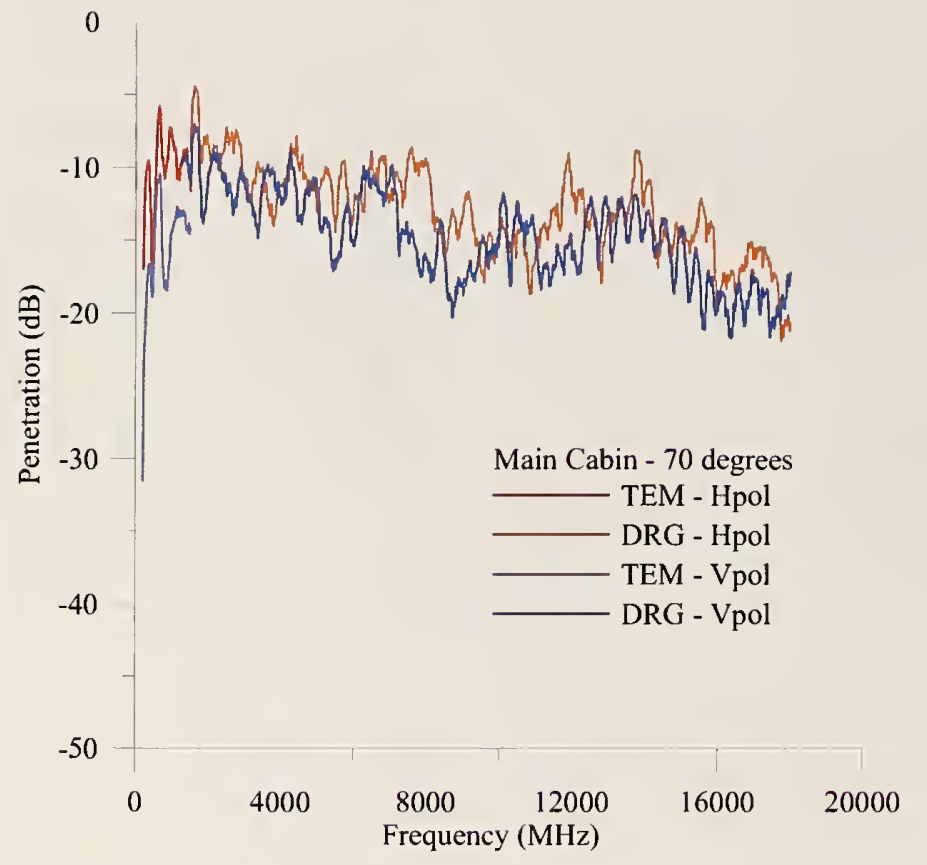

330

$30:$

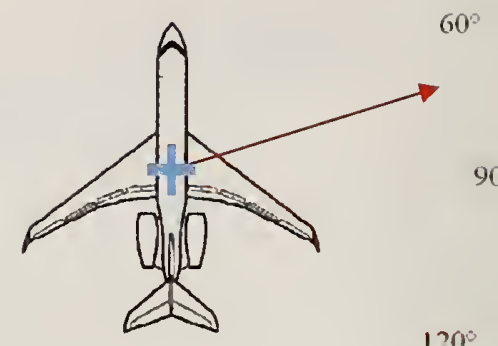

$150^{\circ}$

$180^{\circ}$

Figure 32. Penetration versus frequency for all antennas and polarizations for the transmitting tower placed at the $70^{\circ}$ position and the receiver placed in the main cabin.
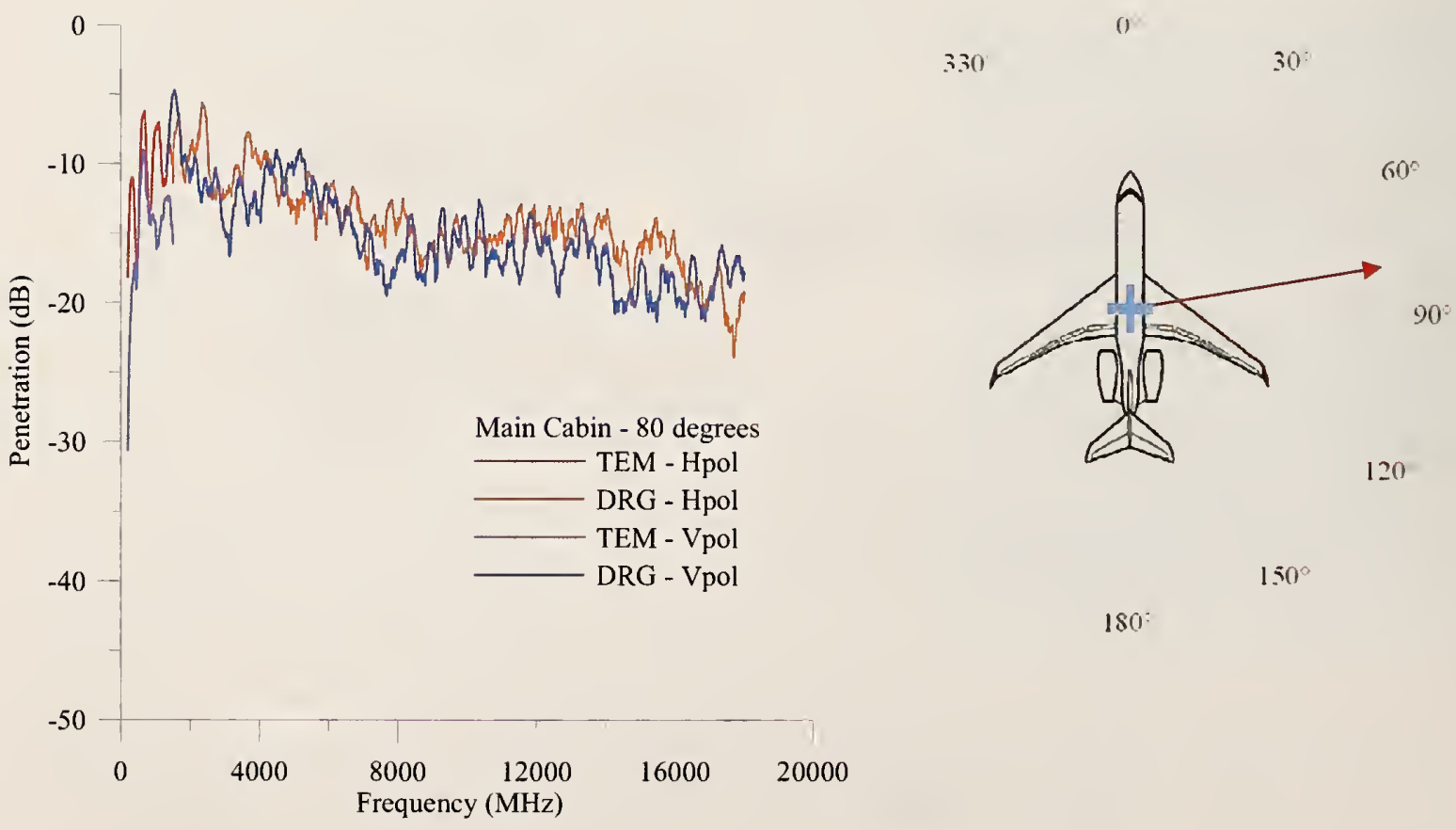

$150^{\circ}$

180 :

Figure 33. Penetration versus frequency for all antennas and polarizations for the transmitting tower placed at the $80^{\circ}$ position and the receiver placed in the main cabin. 


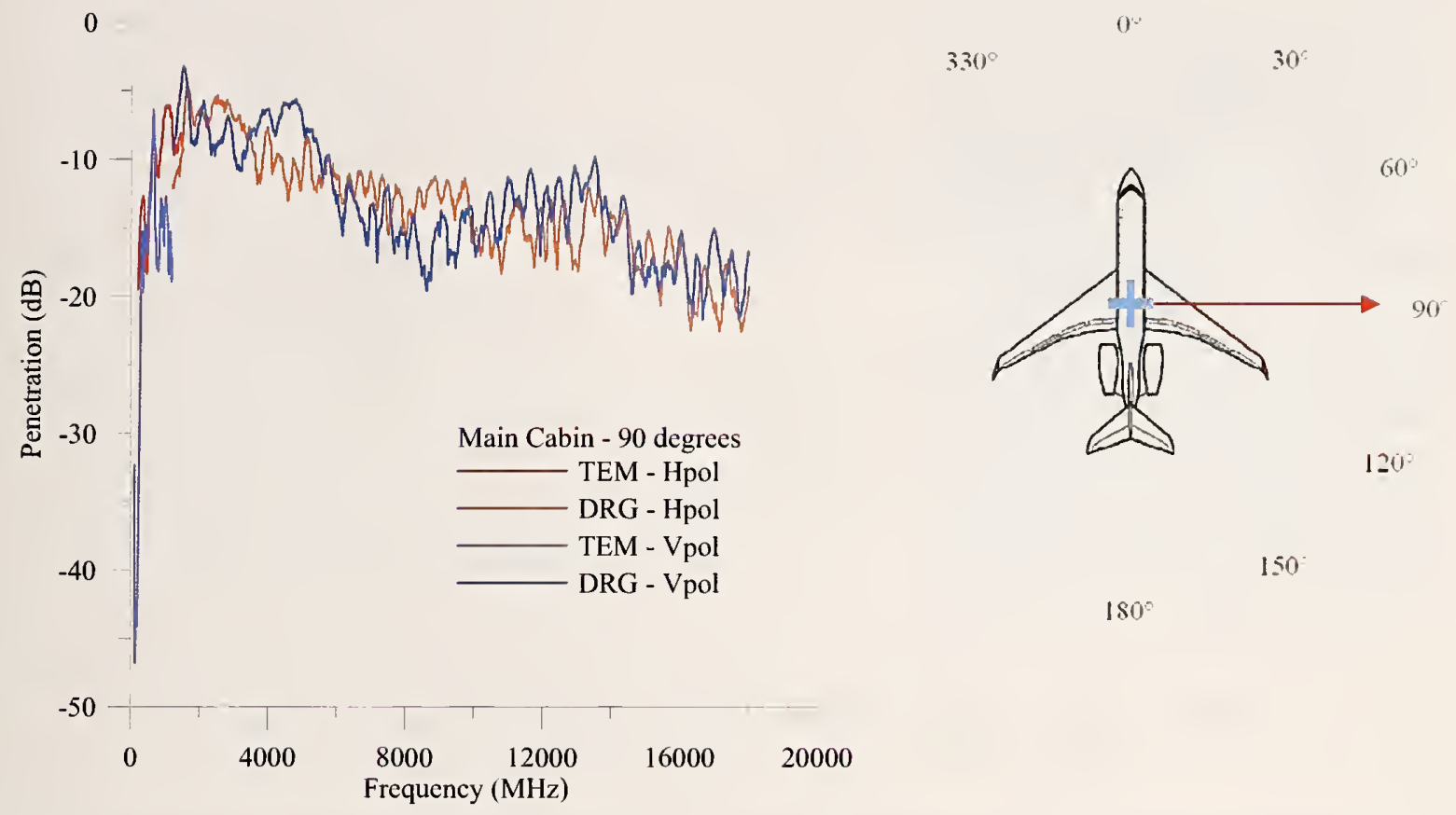

Figure 34. Penetration versus frequency for all antennas and polarizations for the transmitting tower placed at the $90^{\circ}$ position and the receiver placed in the main cabin.
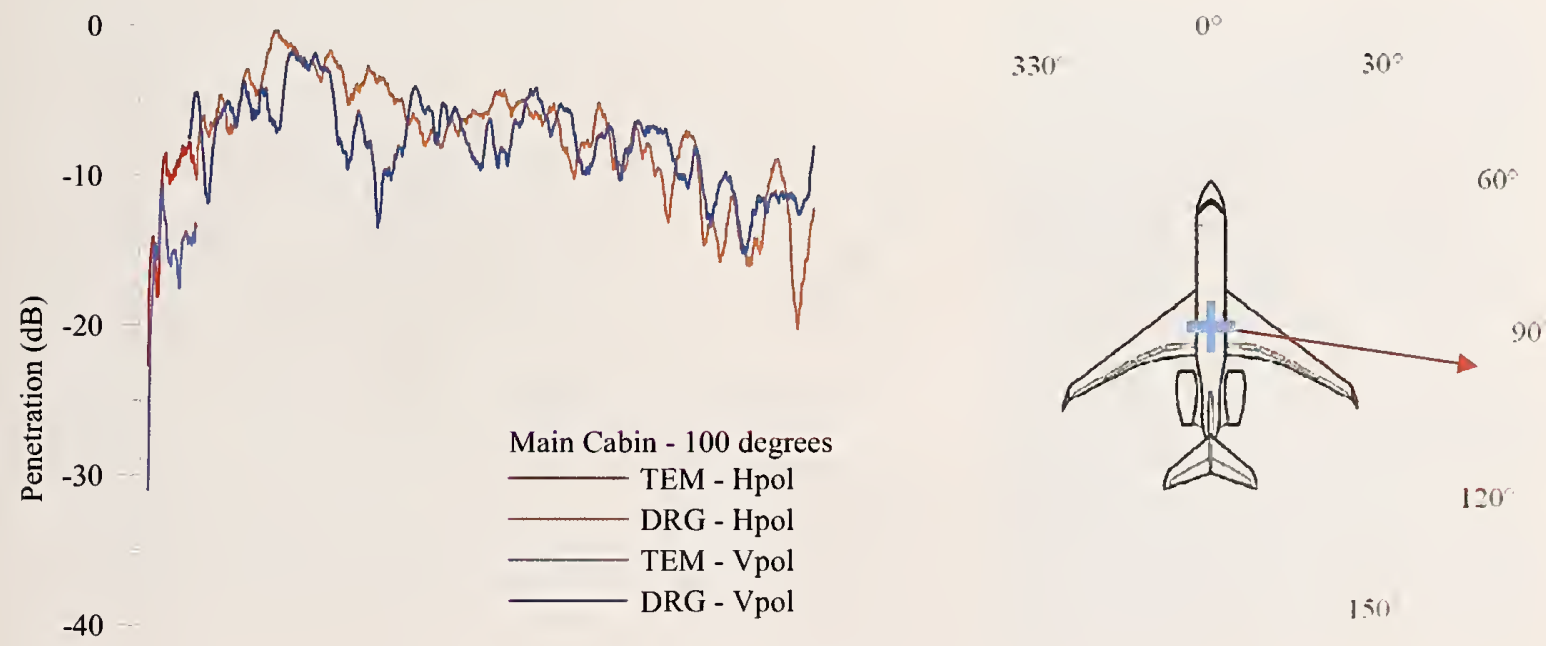

1.50

$150^{\circ}$

$-50$

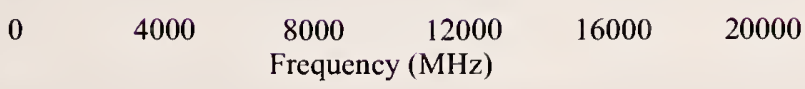

Figure 35. Penetration versus frequency for all antennas and polarizations for the transmitting tower placed at the $100^{\circ}$ position and the receiver placed in the main cabin. 

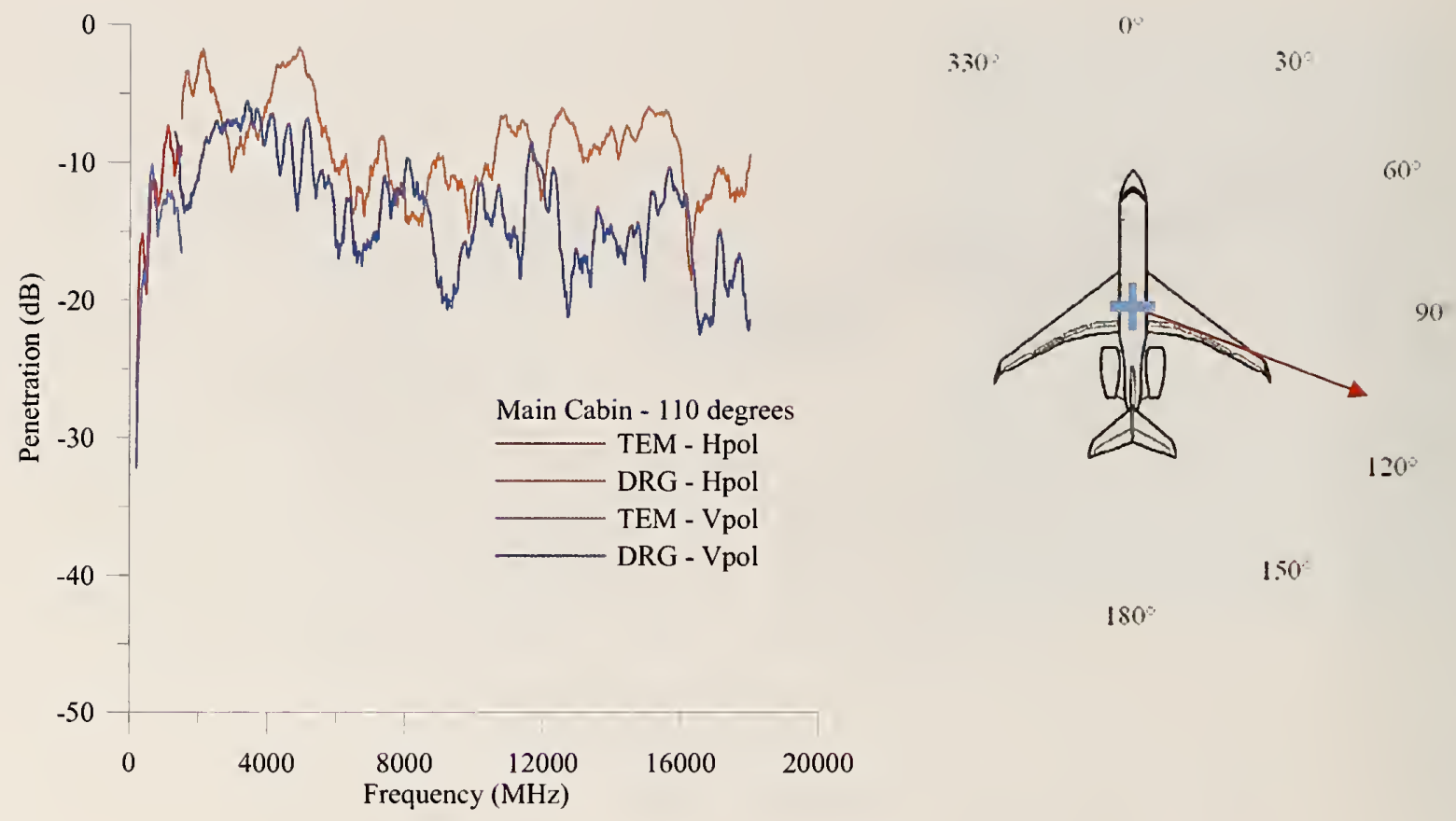

1.50

$180^{\circ}$

Figure 36. Penetration versus frequency for all antennas and polarizations for the transmitting tower placed at the $110^{\circ}$ position and the receiver placed in the main cabin.

0

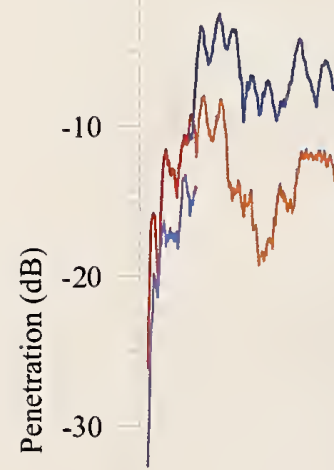

$-40$

$-50$
330

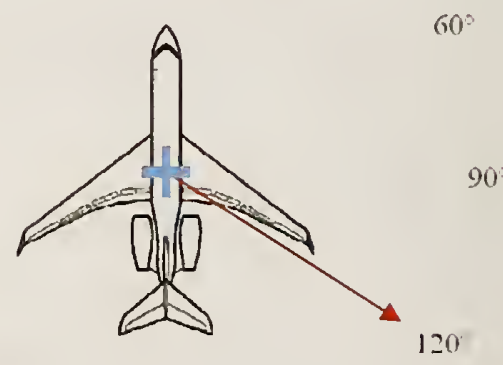

Main Cabin - 120 degrees

TEM - Hpol

DRG - Hpol

TEM - Vpol

DRG - Vpol
30

150 
0

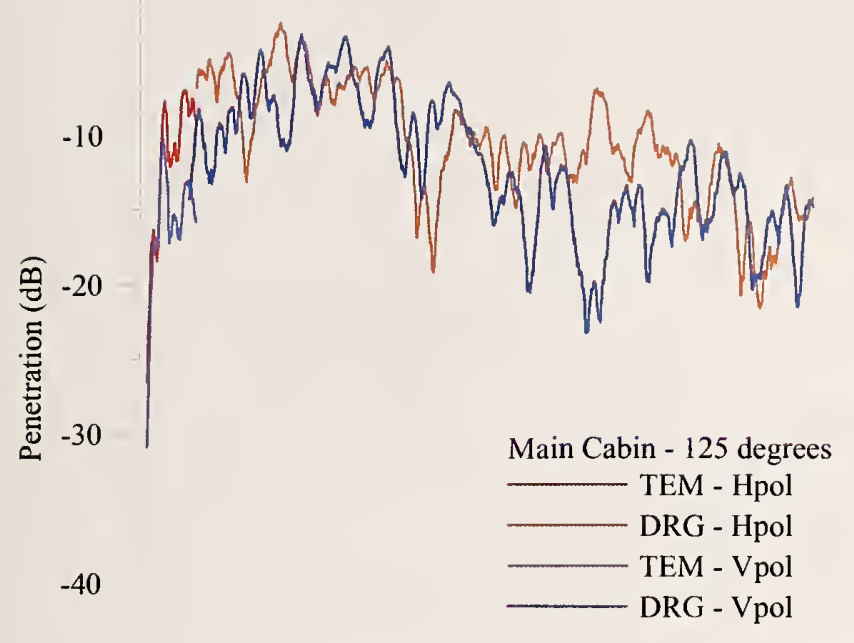

$330^{\circ} \quad 30$

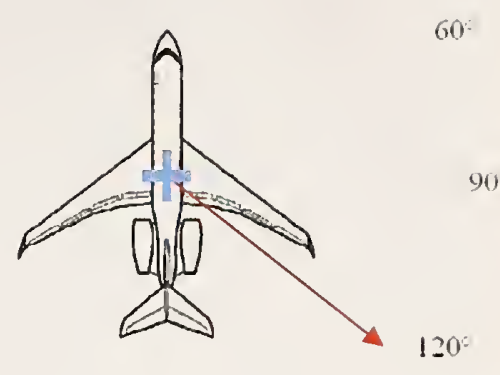

150

$180^{\circ}$

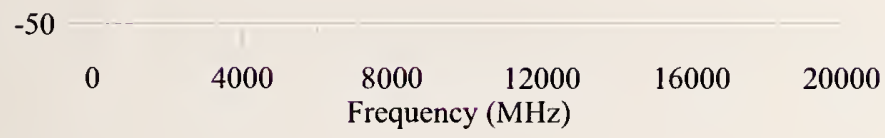

Figure 38. Penetration versus frequency for all antennas and polarizations for the transmitting tower placed at the $125^{\circ}$ position and the receiver placed in the main cabin.

10

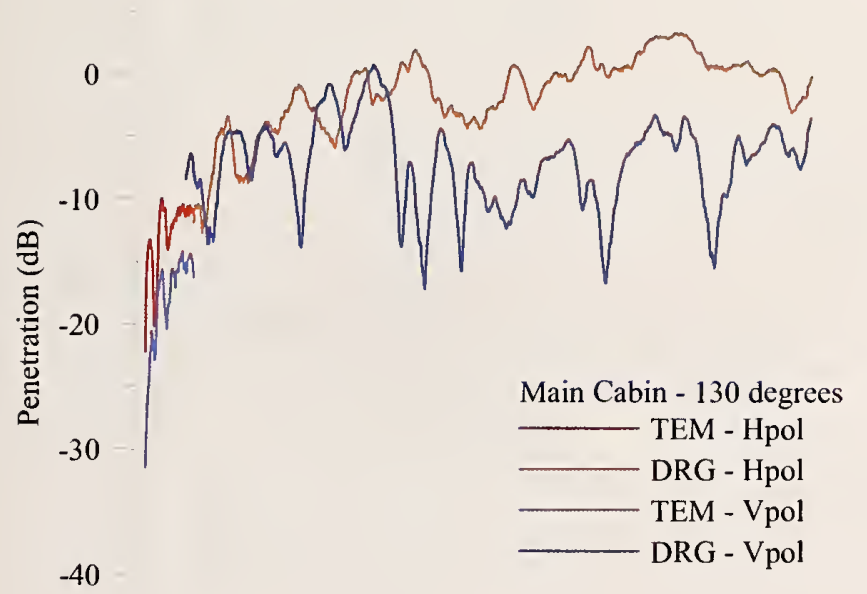

$3.30^{6}$

0
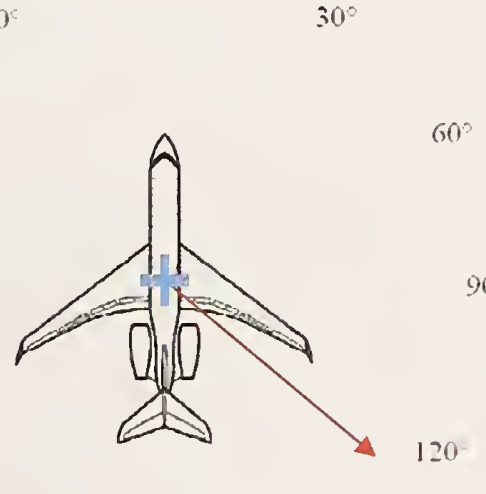

150

$180^{\circ}$

$-50$

$\begin{array}{cccccc}0 & 4000 & 8000 & 12000 & 16000 & 20000 \\ & & \text { Frequency }(\mathrm{MHz})\end{array}$

Figure 39. Penetration versus frequency for all antennas and polarizations for the transmitting tower placed at the $130^{\circ}$ position and the receiver placed in the main cabin. 


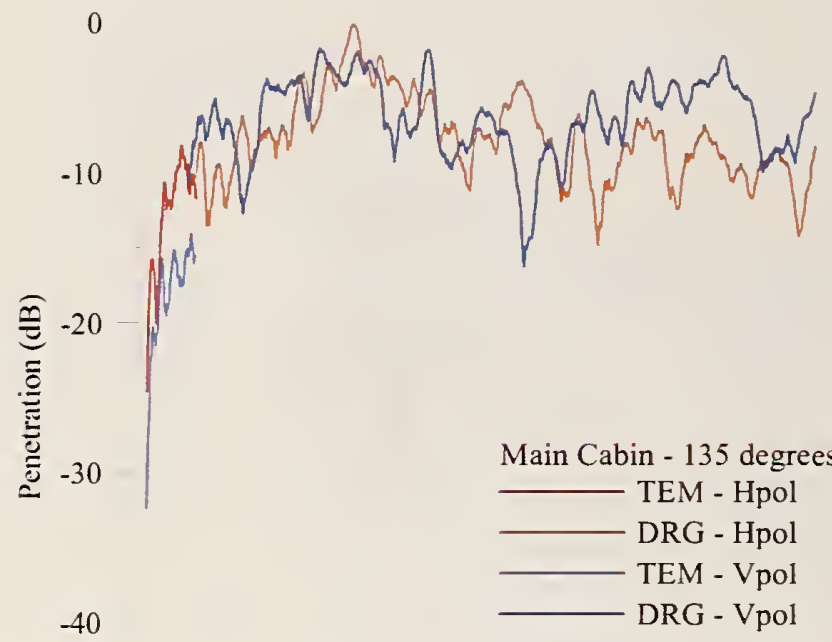

3.30

30

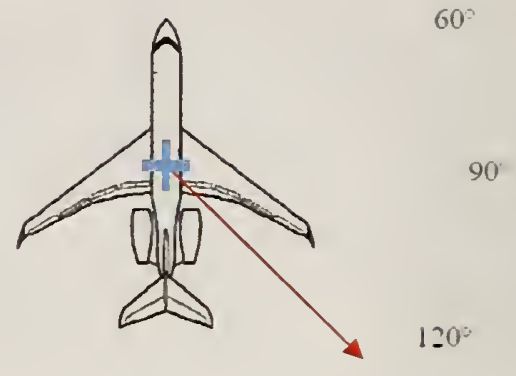

150

$180^{\circ}$

$-50$

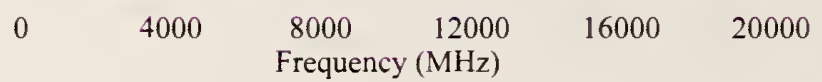

Figure 40. Penetration versus frequency for all antennas and polarizations for the transmitting tower placed at the $135^{\circ}$ position and the receiver placed in the main cabin.

10

$330 \quad 30^{\circ}$

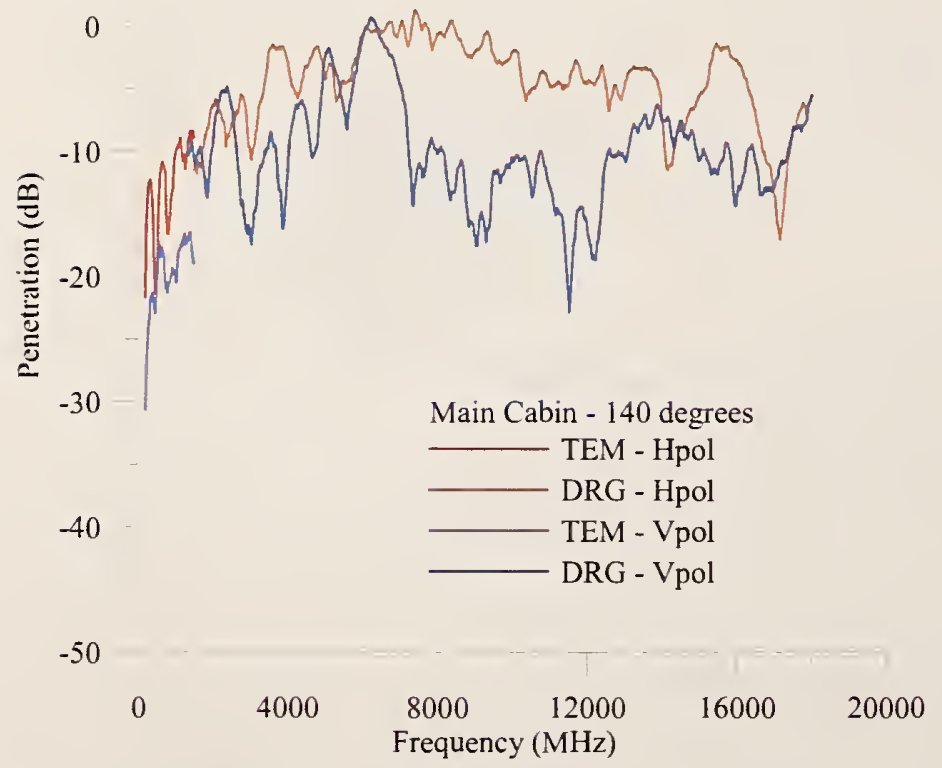

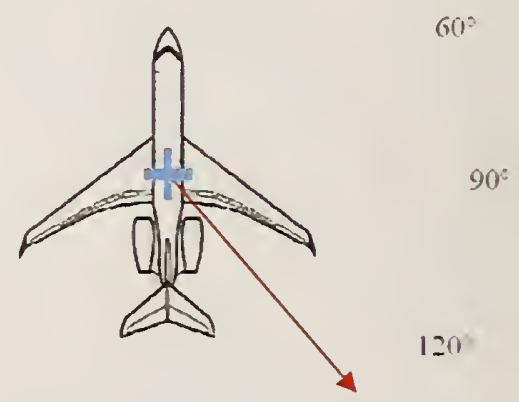

150

$180^{\circ}$

Figure 41. Penetration versus frequency for all antennas and polarizations for the transmitting tower placed at the $140^{\circ}$ position and the receiver placed in the main cabin. 

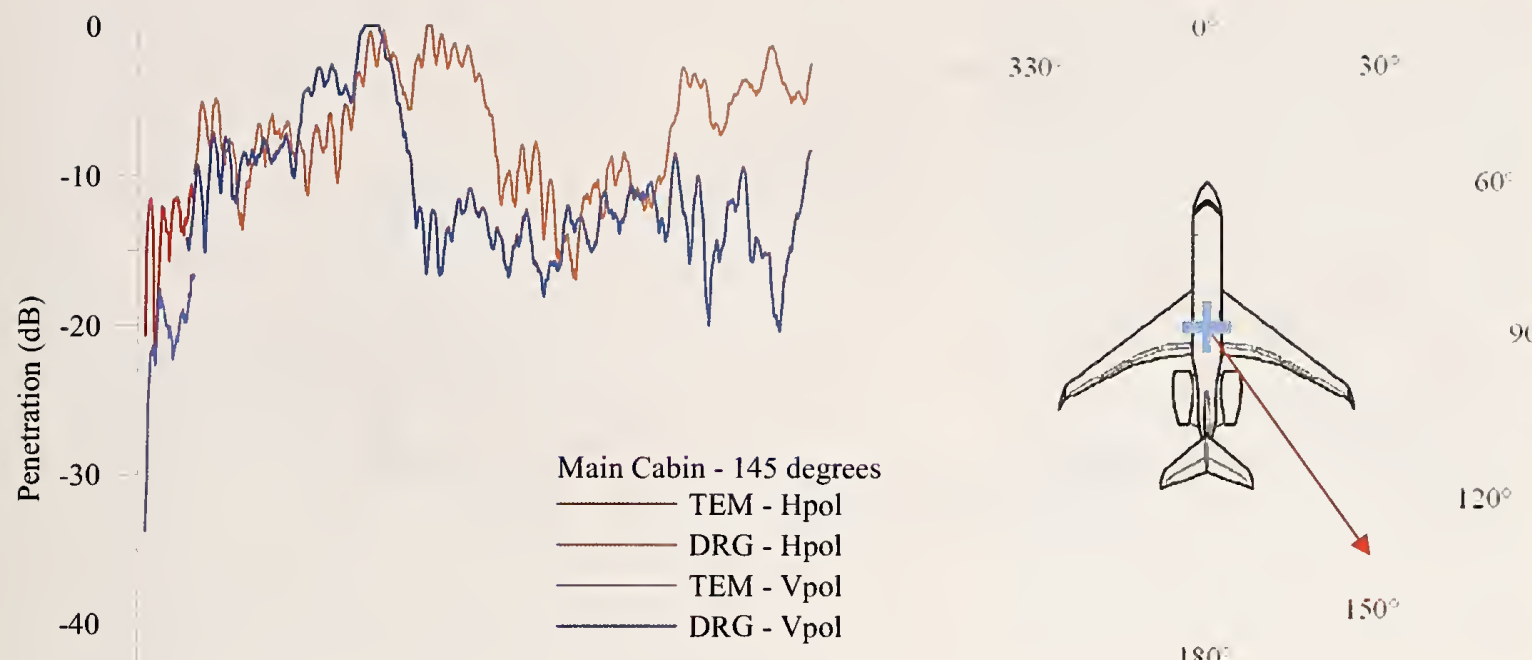

180

$-50$

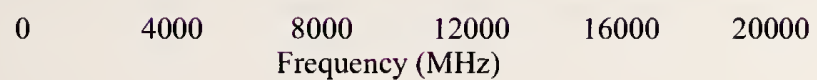

Figure 42. Penetration versus frequency for all antennas and polarizations for the transmitting tower placed at the $145^{\circ}$ position and the receiver placed in the main cabin.

0

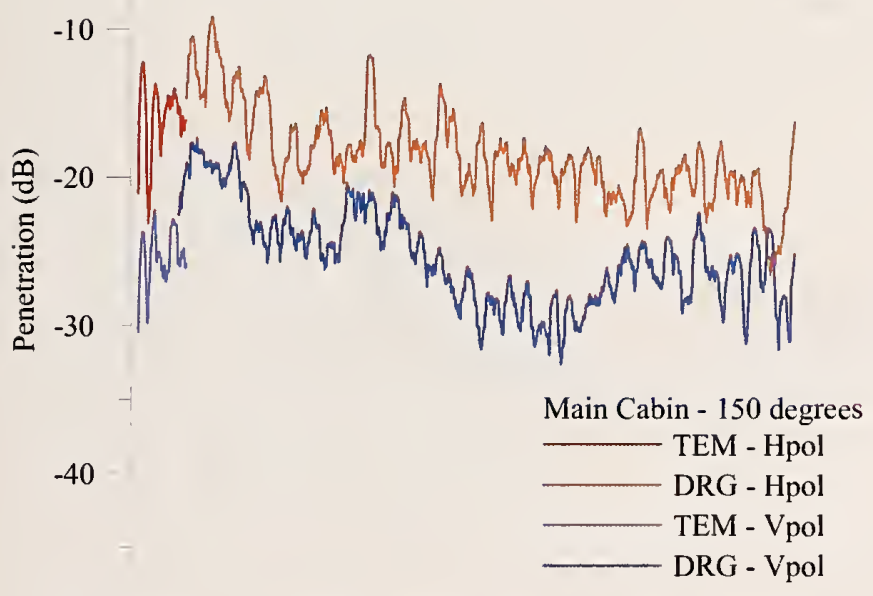

$-50$ $330^{\circ}$

()

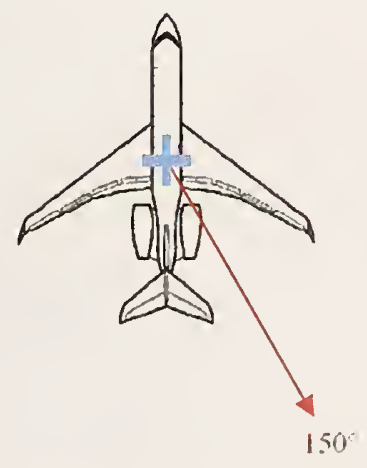

$180^{\circ}$

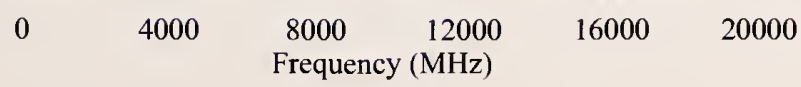

Figure 43. Penetration versus frequency for all antennas and polarizations for the transmitting tower placed at the $150^{\circ}$ position and the receiver placed in the main cabin. 
10

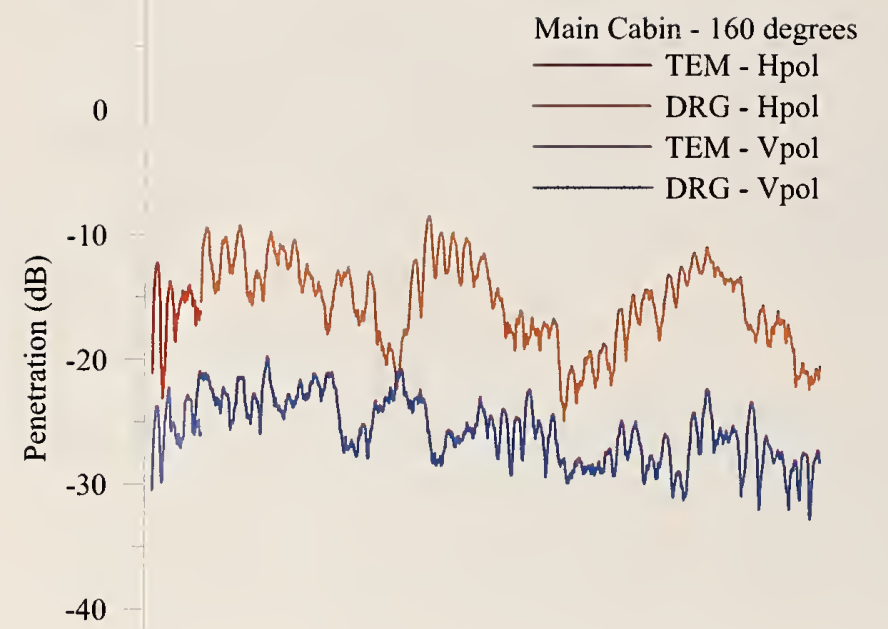

3.30

()

$-40$

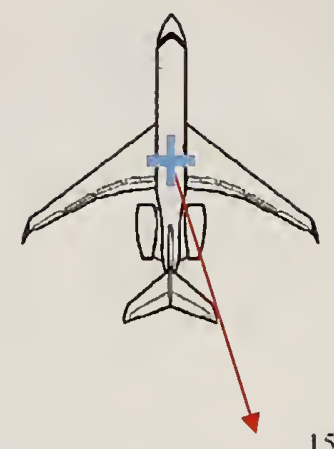

60

90

$180^{\circ}$

$-50$

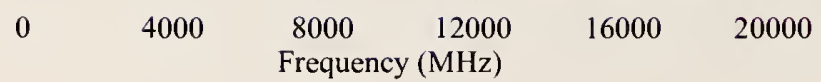

Figure 44. Penetration versus frequency for all antennas and polarizations for the transmitting tower placed at the $160^{\circ}$ position and the receiver placed in the main cabin.

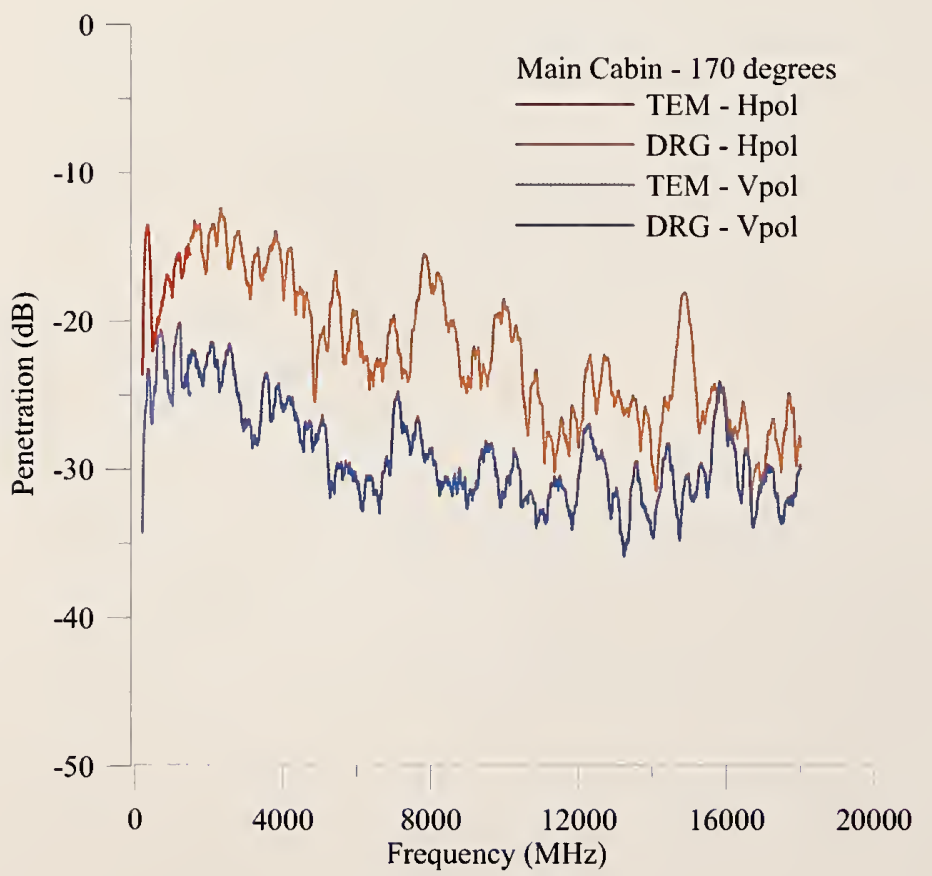

$60^{\circ}$

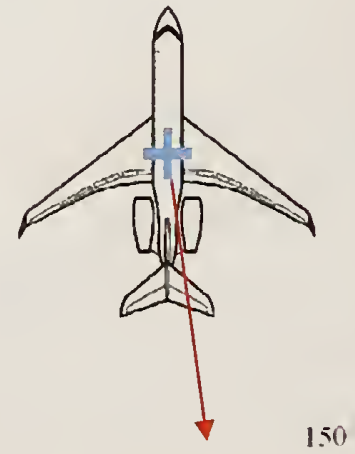

$180^{\circ}$

Figure 45. Penetration versus frequency for all antennas and polarizations for the transmitting tower placed at the $170^{\circ}$ position and the receiver placed in the main cabin. 
0

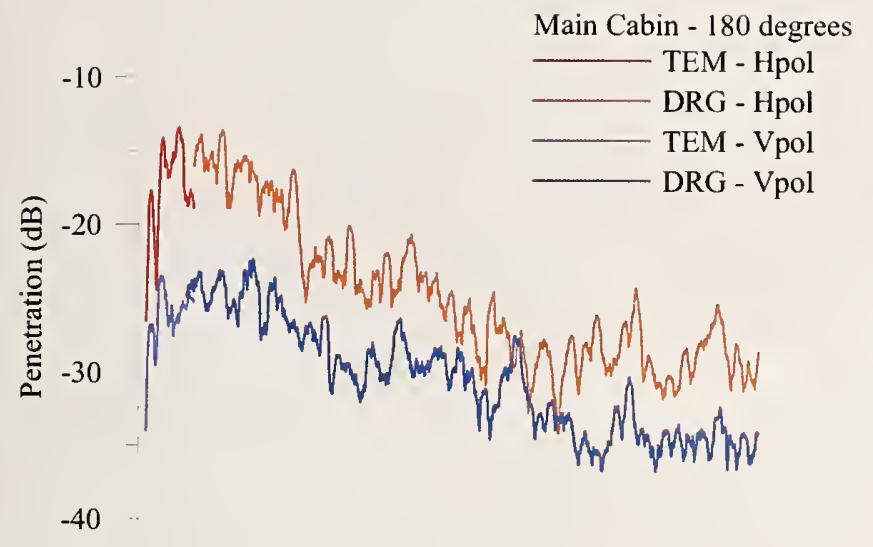

$-40$

$-50$

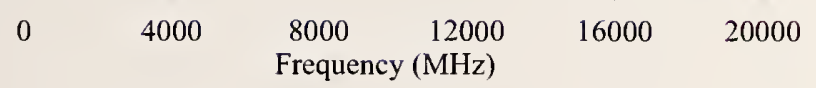

330

0

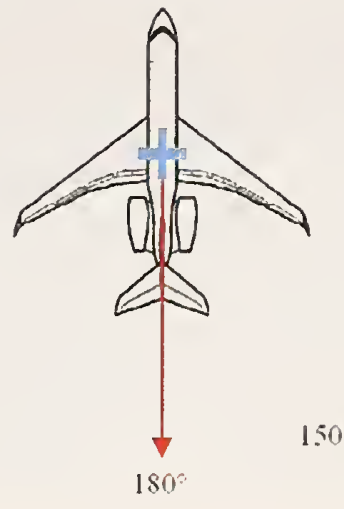

60

Figure 46. Penetration versus frequency for all antennas and polarizations for the transmitting tower placed at the $180^{\circ}$ position and the receiver placed in the main cabin.

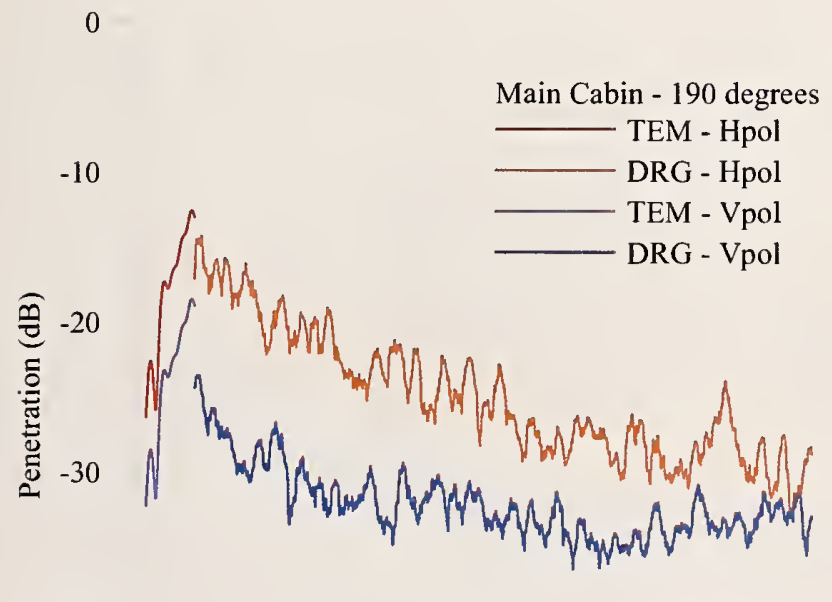

$-40$

$-50$
3.30 $30=$

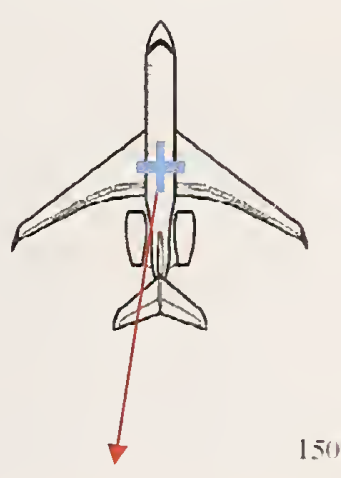

$180^{\circ}$ $60^{\circ}$

Figure 47. Penetration versus frequency for all antennas and polarizations for the transmitting tower placed at the $190^{\circ}$ position and the receiver placed in the main cabin. 

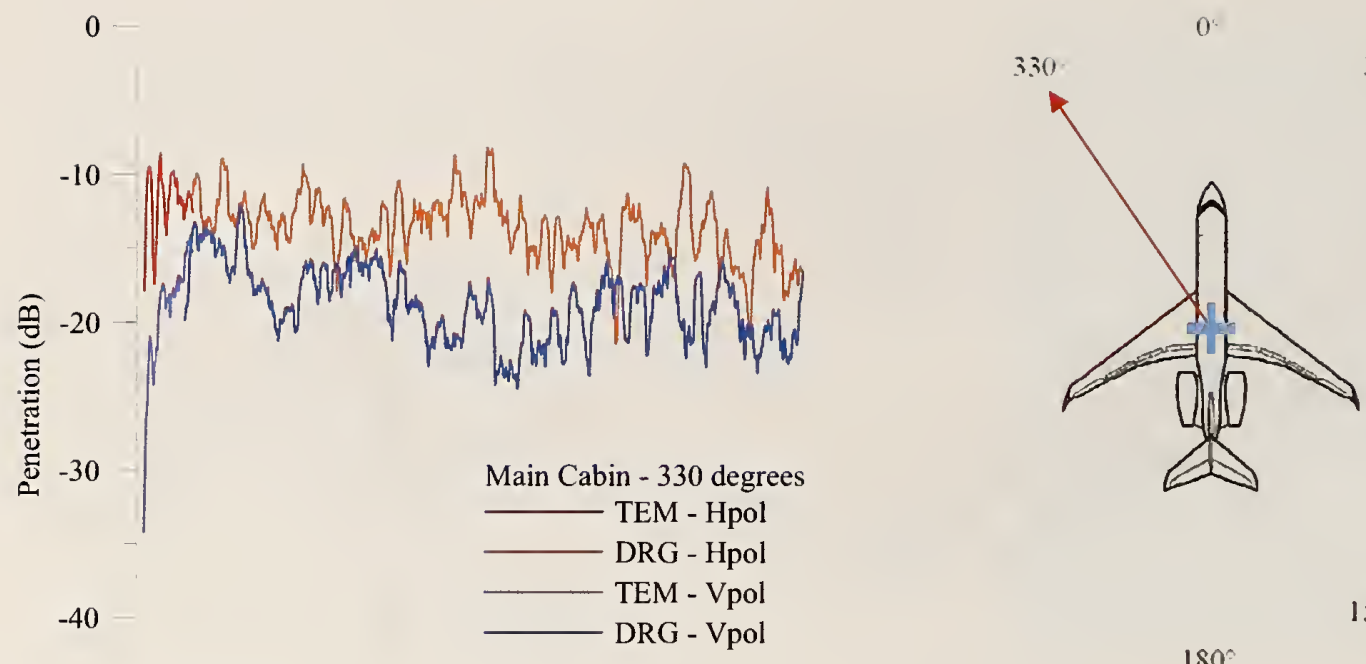

$30^{\circ}$

$60^{3}$

90

$120^{\circ}$

150

$180^{\circ}$

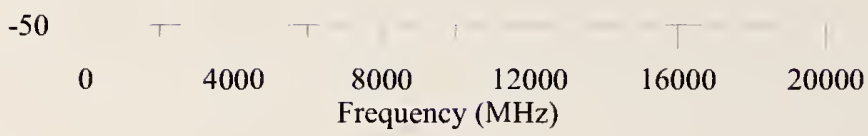

Figure 48. Penetration versus frequency for all antennas and polarizations for the transmitting tower placed at the $330^{\circ}$ position and the receiver placed in the main cabin.
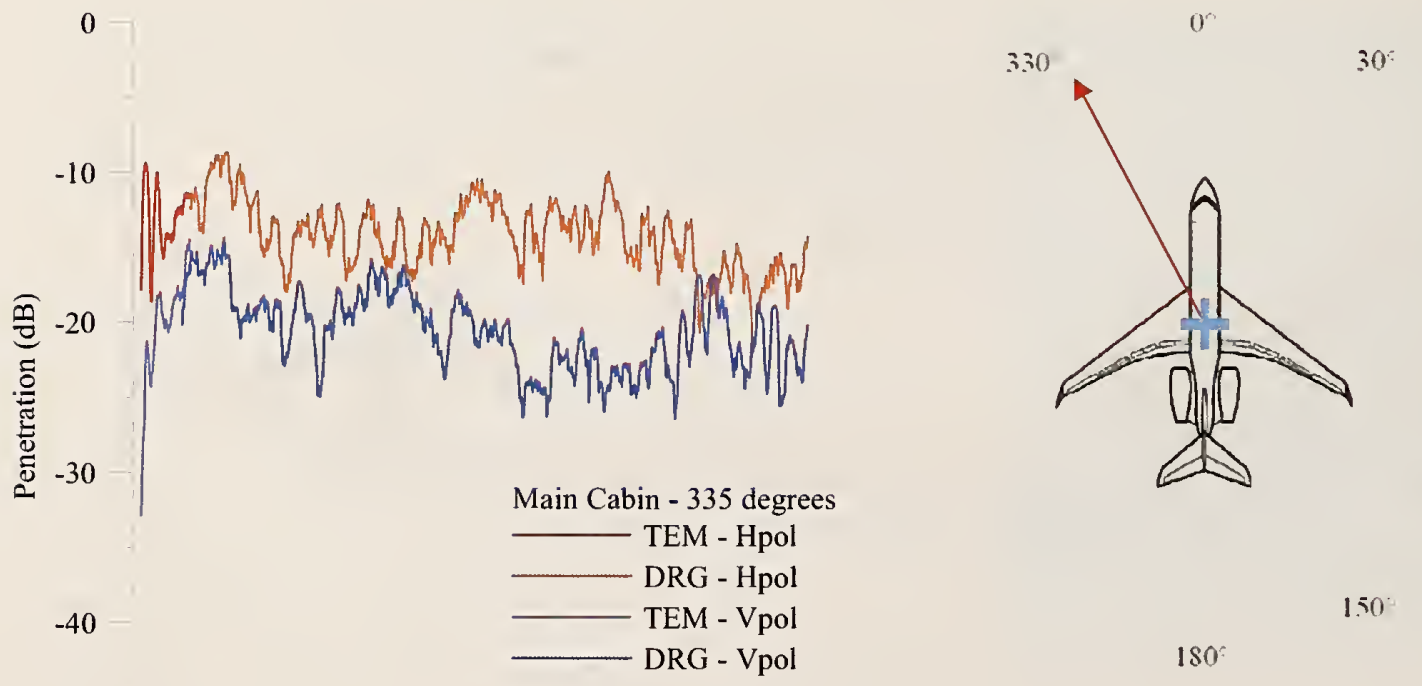

$60^{\circ}$

$90^{\circ}$

$-50$

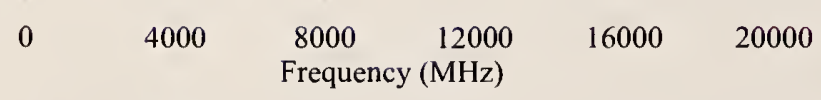

Figure 49. Penetration versus frequency for all antennas and polarizations for the transmitting tower placed at the $335^{\circ}$ position and the receiver placed in the main cabin. 

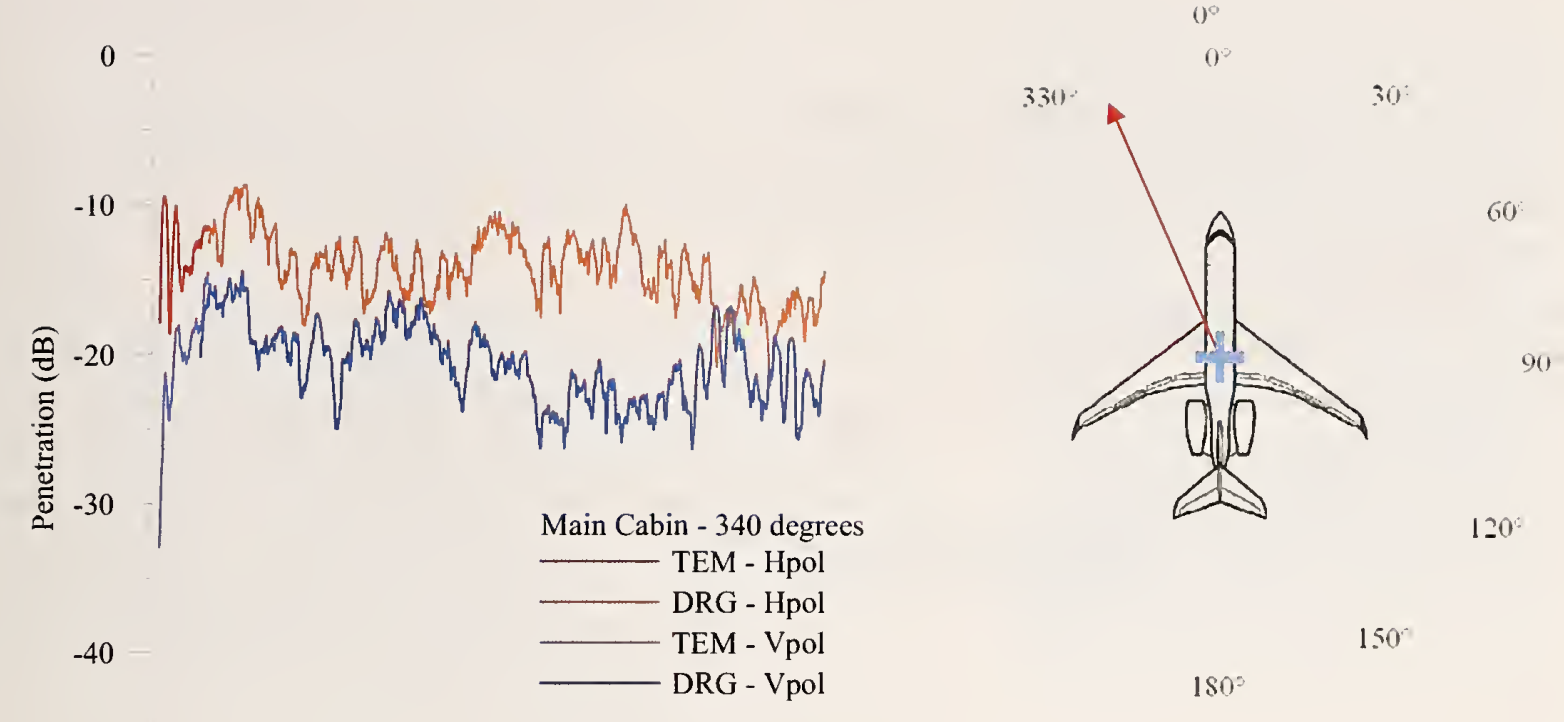

$-50$

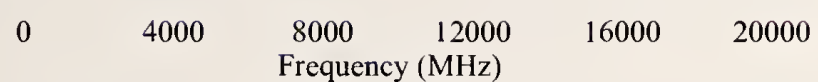

Figure 50. Penetration versus frequency for all antennas and polarizations for the transmitting tower placed at the $340^{\circ}$ position and the receiver placed in the main cabin.

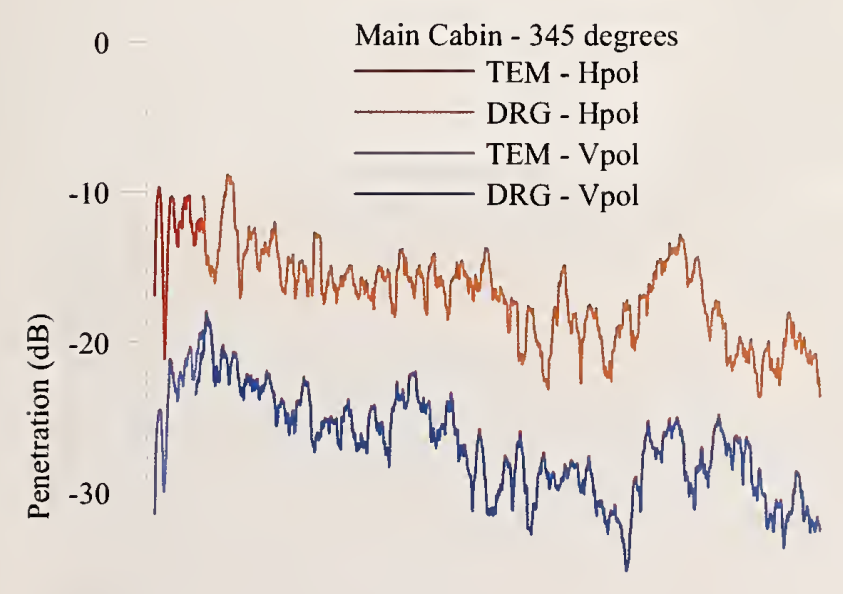

$-40$

$-50$

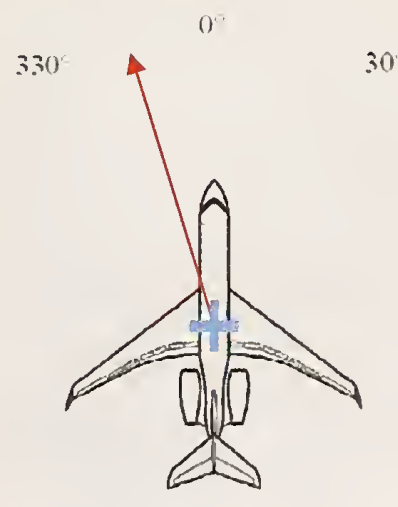

$60^{\circ}$
1.50

$180^{\circ}$

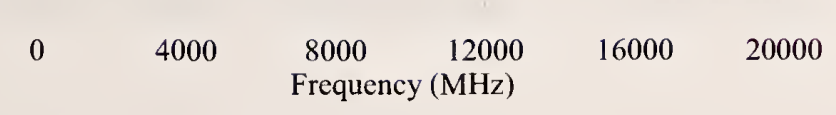

Figure 51. Penetration versus frequency for all antennas and polarizations for the transmitting tower placed at the $345^{\circ}$ position and the receiver placed in the main cabin. 

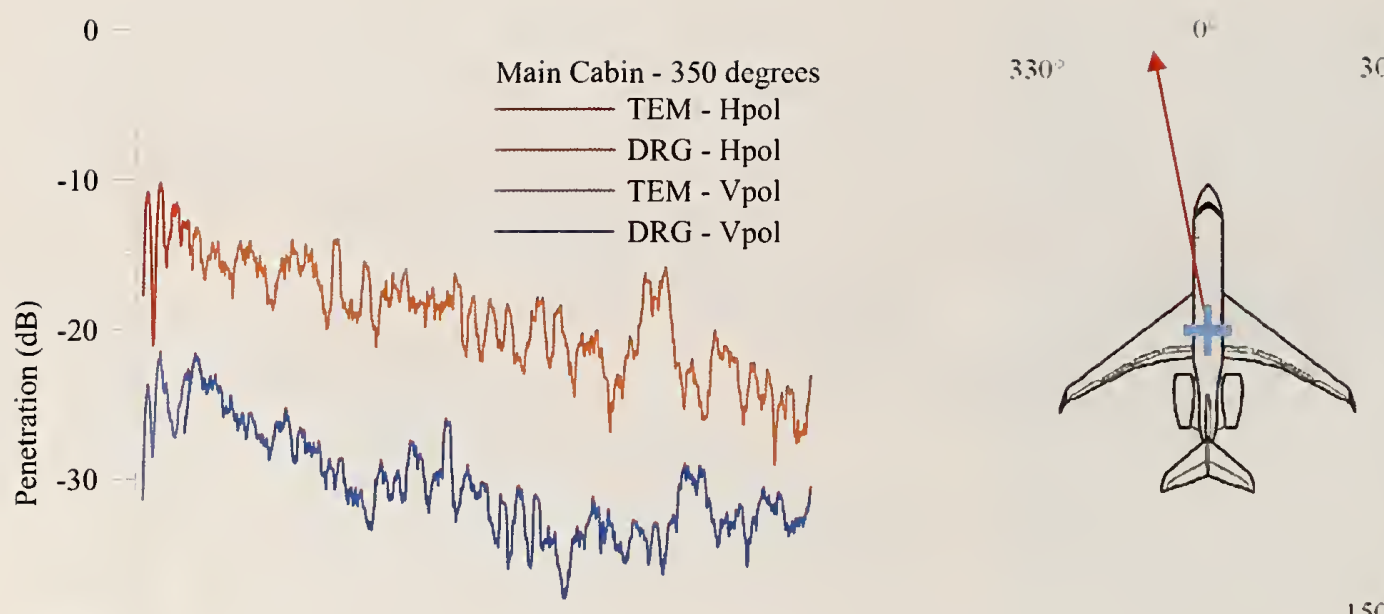

60

90

$120^{\circ}$

$-40$

$150^{\circ}$

$180^{\circ}$

$-50$

$\begin{array}{cccccc}0 & 4000 & 8000 & 12000 & 16000 & 20000 \\ & & \text { Frequency }(\mathrm{MHz})\end{array}$

Figure 52. Penetration versus frequency for all antennas and polarizations for the transmitting tower placed at the $350^{\circ}$ position and the receiver placed in the main cabin.
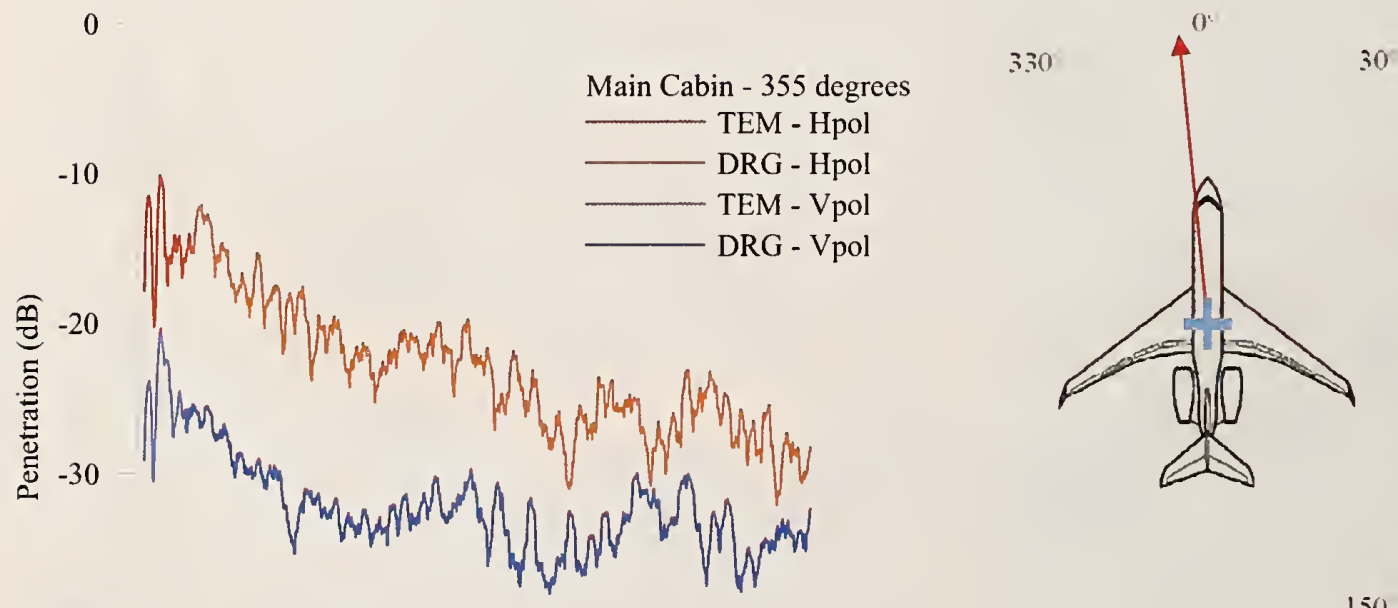

$60^{\circ}$

$90^{\circ}$

150

$180^{\circ}$

$-50$

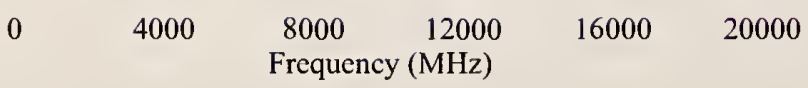

Figure 53. Penetration versus frequency for all antennas and polarizations for the transmitting tower placed at the $355^{\circ}$ position and the receiver placed in the main cabin. 


\subsection{Flight Deck Penetration Results}

The second penetration study was performed with the receiving antennas placed in the flight deck between the pilot's and the co-pilot's seats, as shown in Figure 54. The time-domain plots for various angular positions around the aircraft are shown in Figure 55. Only the horizontal polarization is shown for the DRG antennas, and we notice that no significant direct coupling components are visible for any position. Therefore, maximum penetration results are lower than for positions in the main cabin, where direct coupling is seen in the time-domain plots. Figure 56 shows penetration data for various positions around the aircraft. Maximum penetration of approximately $-15 \mathrm{~dB}$ occurs around the nose position for the vertical polarization, but decays rapidly for the horizontal position at $0^{\circ}$ and at frequencies above $2000 \mathrm{MHz}$. Minimum penetration of $-45 \mathrm{~dB}$ occurs at the $190^{\circ}$ position for both polarizations. Symmetry around the aircraft is shown in Figure 57. Figures 58 to 86 show detailed penetration data for each individual external position around the aircraft.

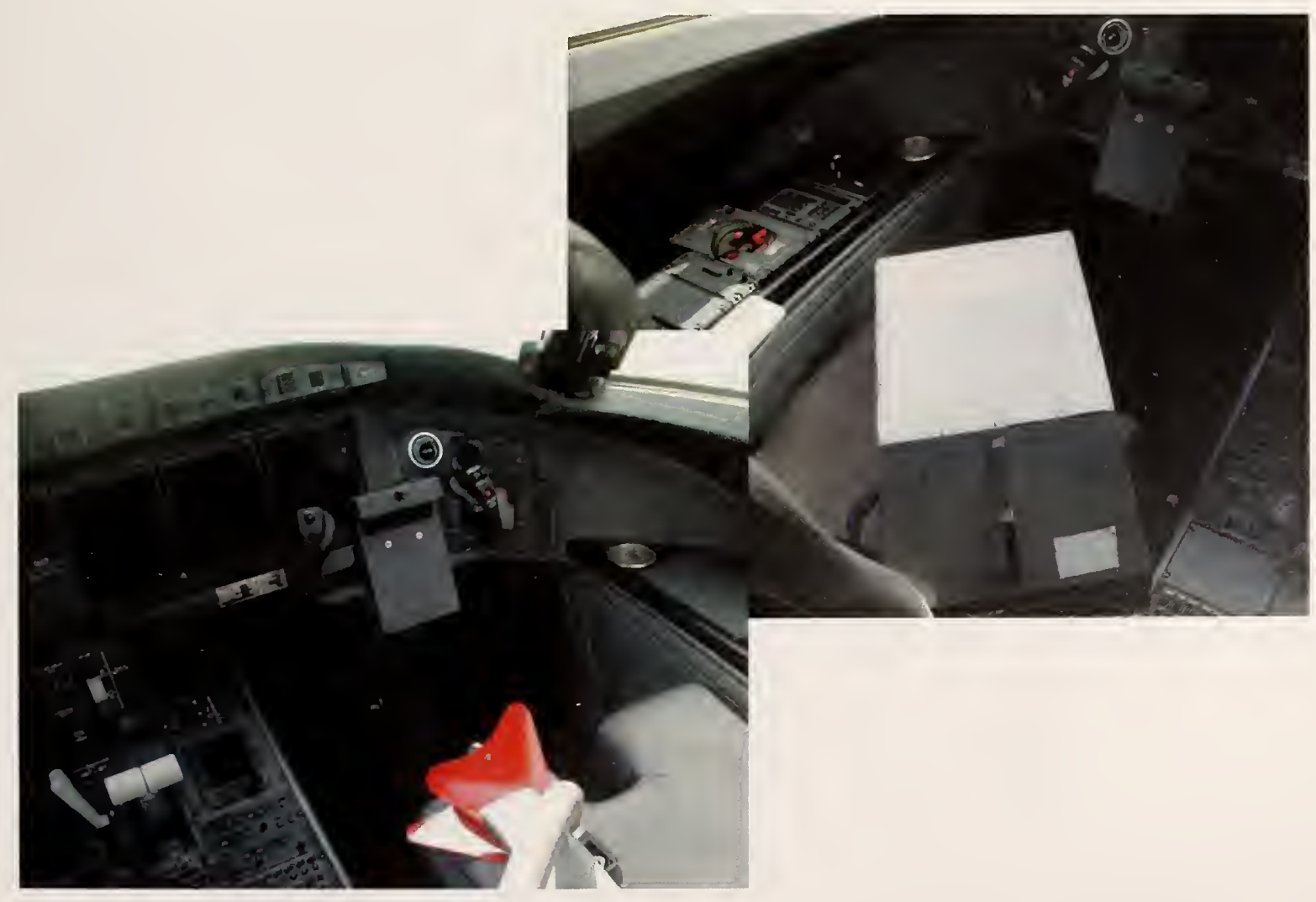

Figure 54. The receiving DRG and TEM antennas placed between the pilot and co-pilot seat as shown in these two photos. 


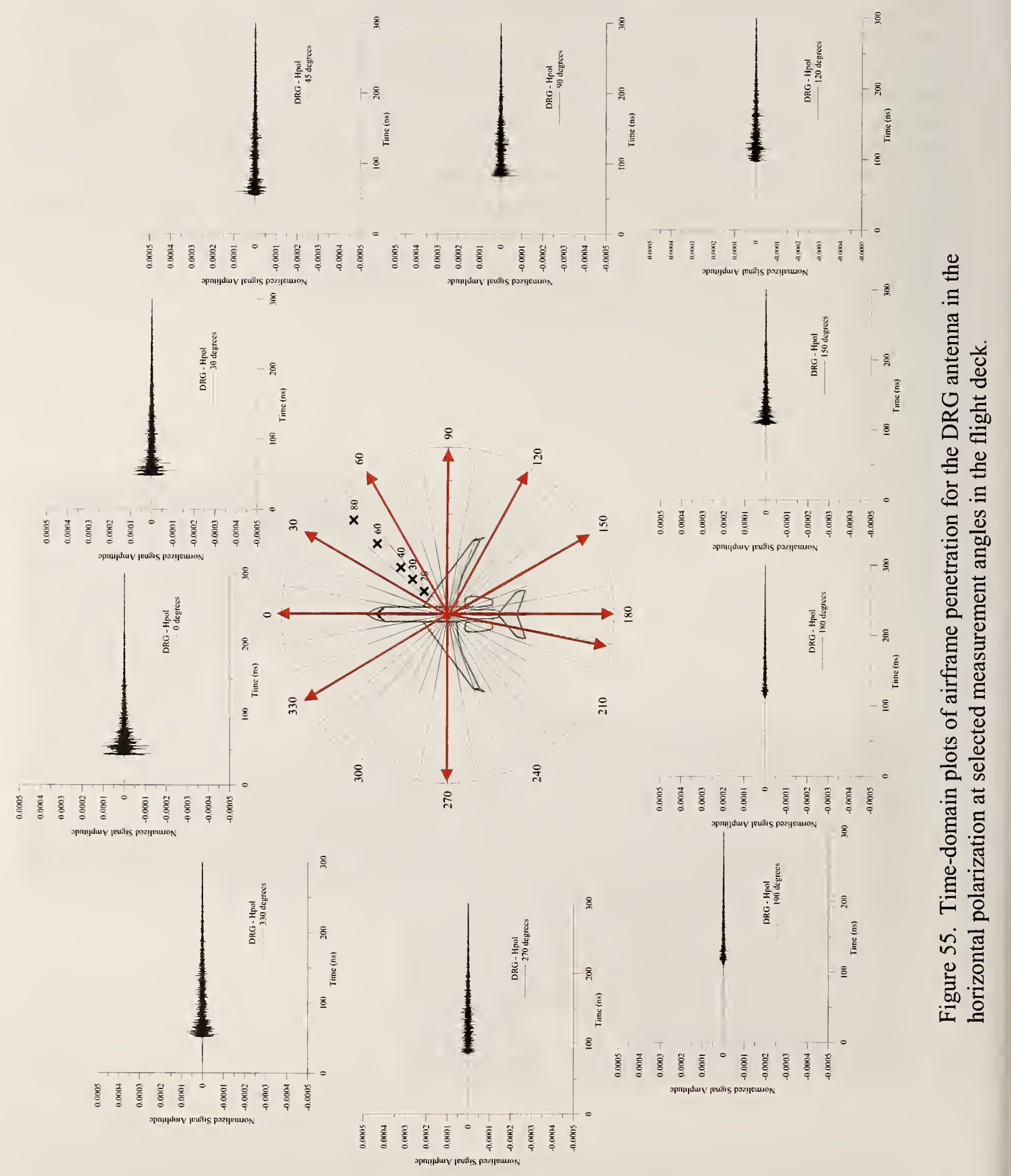




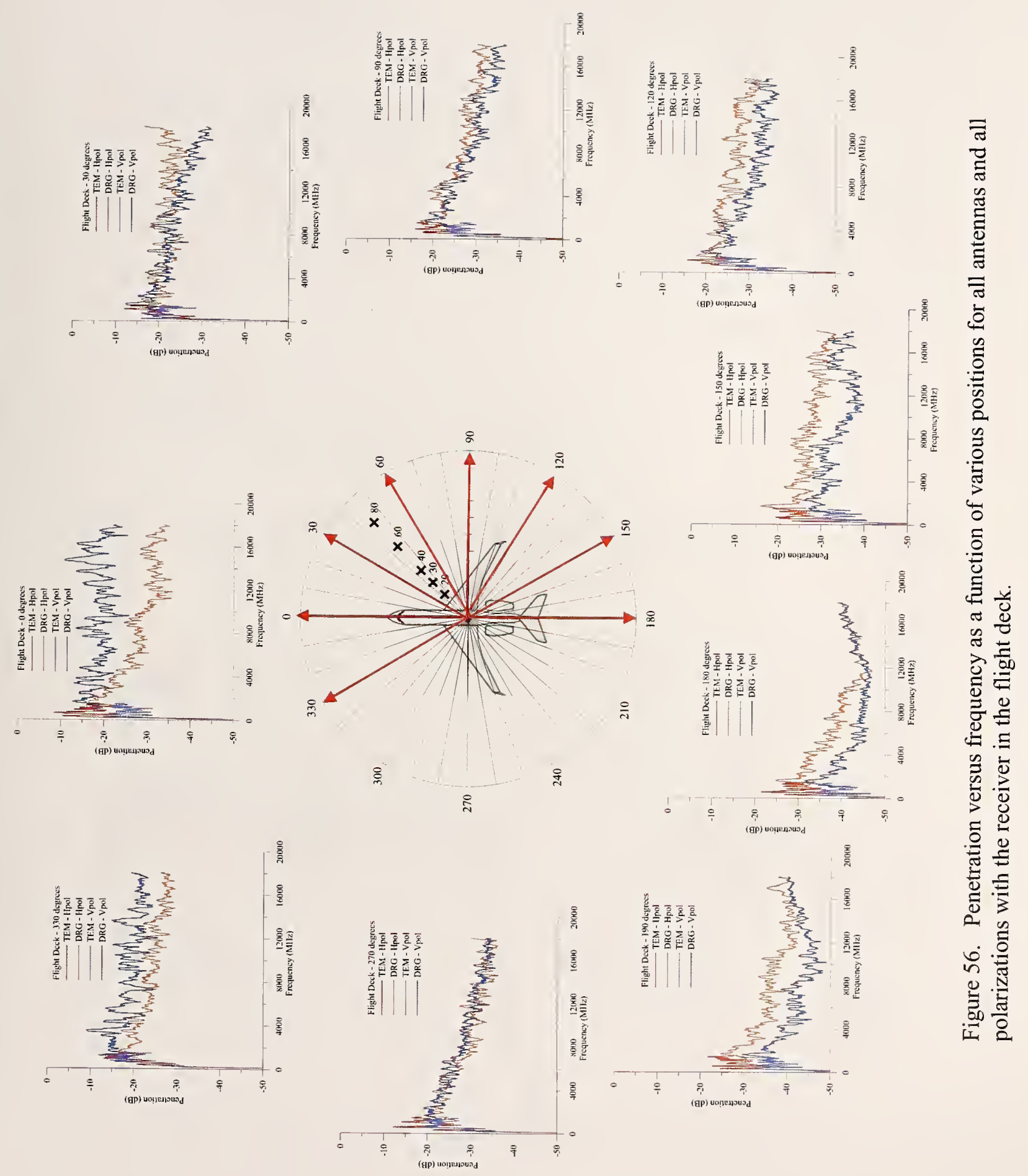

43 


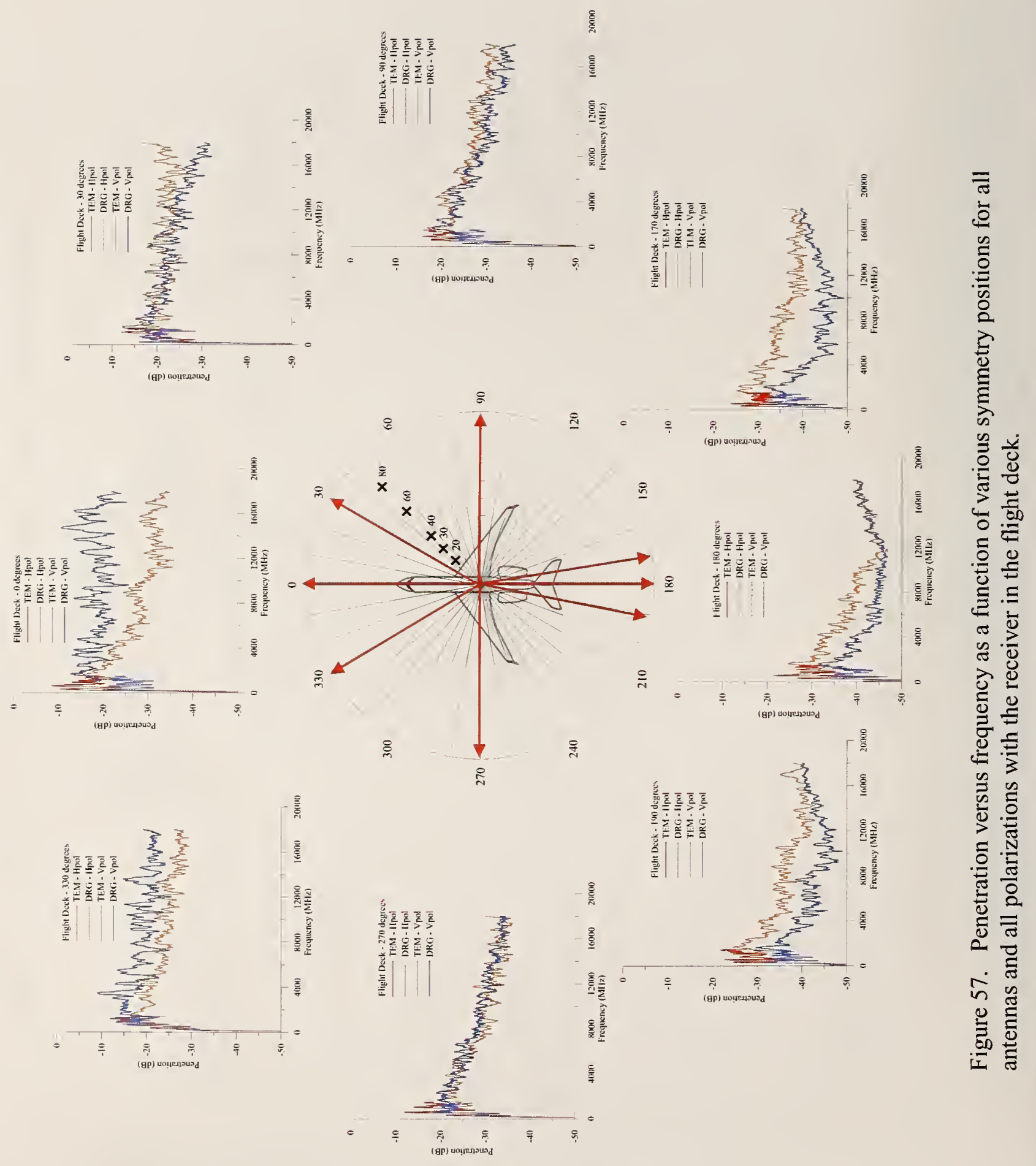




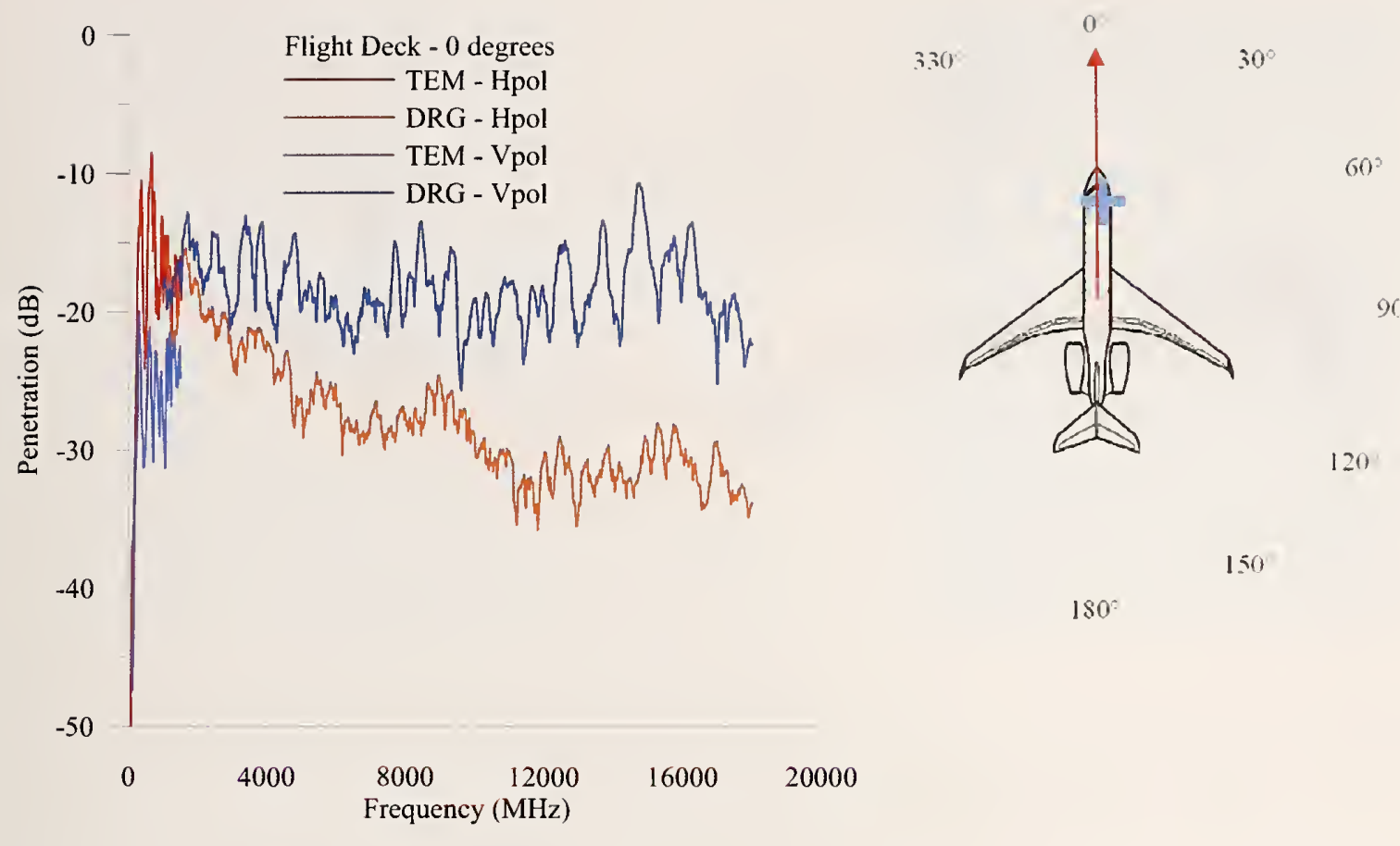

Figure 58. Penetration versus frequency for all antennas and polarizations for the transmitting tower placed at the $0^{\circ}$ position and the receiver placed in the flight deck.

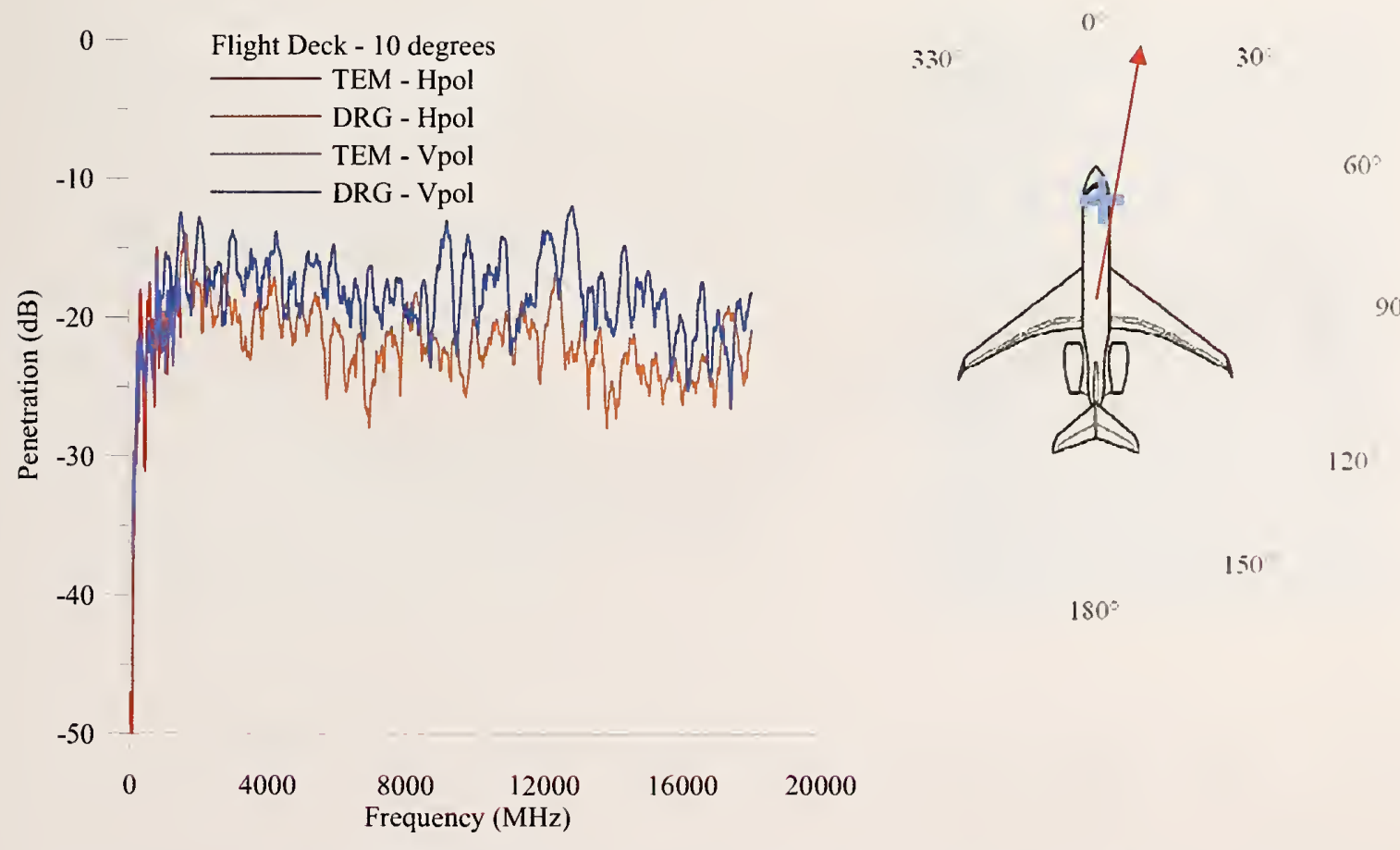

Figure 59. Penetration versus frequency for all antennas and polarizations for the transmitting tower placed at the $10^{\circ}$ position and the receiver placed in the flight deck. 


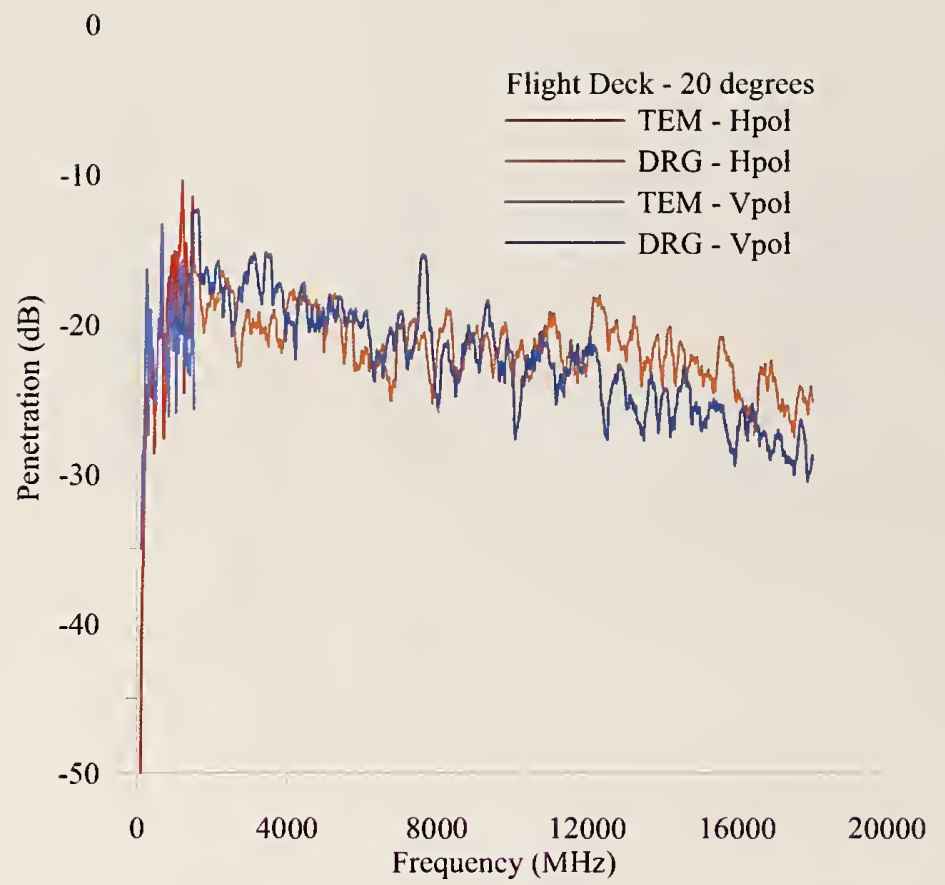

330

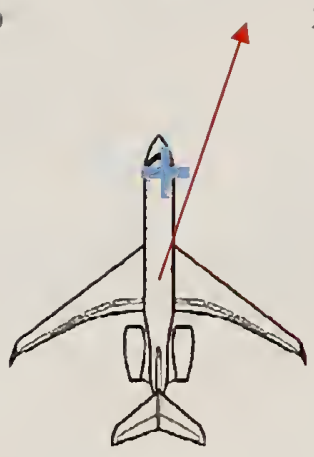

$\$ 0$

60

$90^{\circ}$

120

1.50

$180^{\circ}$

Figure 60. Penetration versus frequency for all antennas and polarizations for the transmitting tower placed at the $20^{\circ}$ position and the receiver placed in the flight deck.
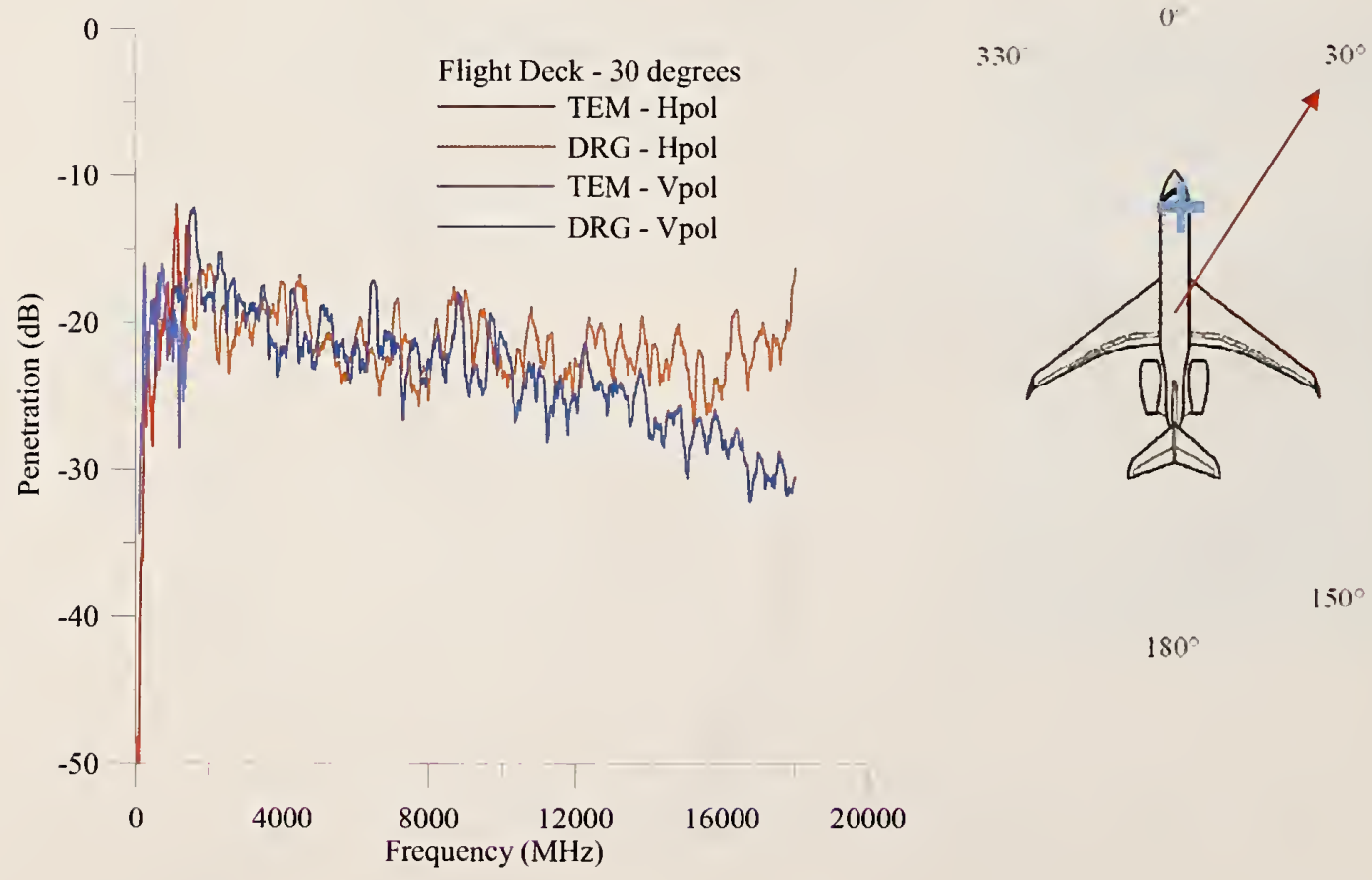

60

$90^{2}$

$180^{\circ}$

120

Figure 61. Penetration versus frequency for all antennas and polarizations for the transmitting tower placed at the $30^{\circ}$ position and the receiver placed in the flight deck. 
0

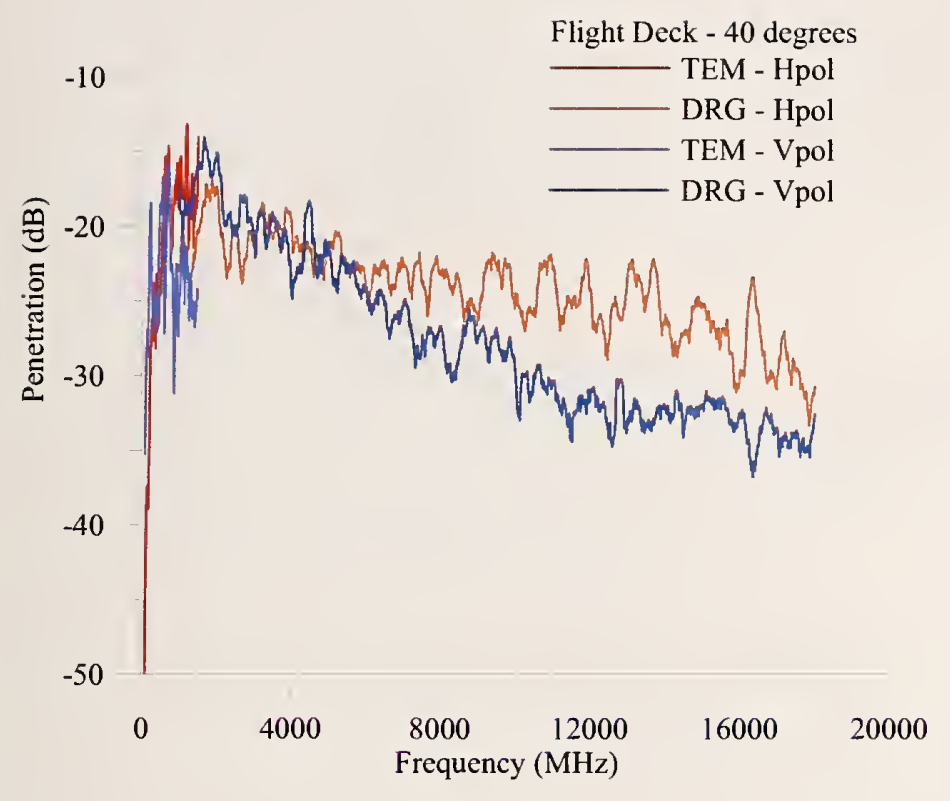

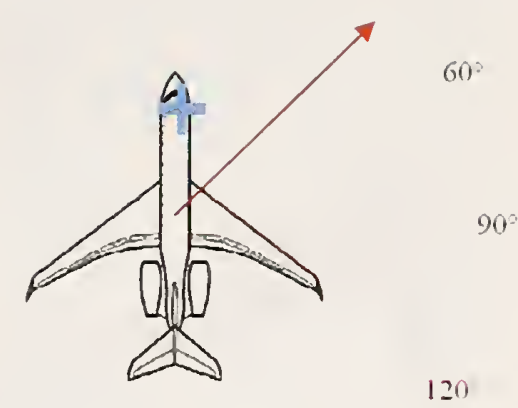

120

$180^{\circ}$

Figure 62. Penetration versus frequency for all antennas and polarizations for the transmitting tower placed at the $40^{\circ}$ position and the receiver placed in the flight deck.

0

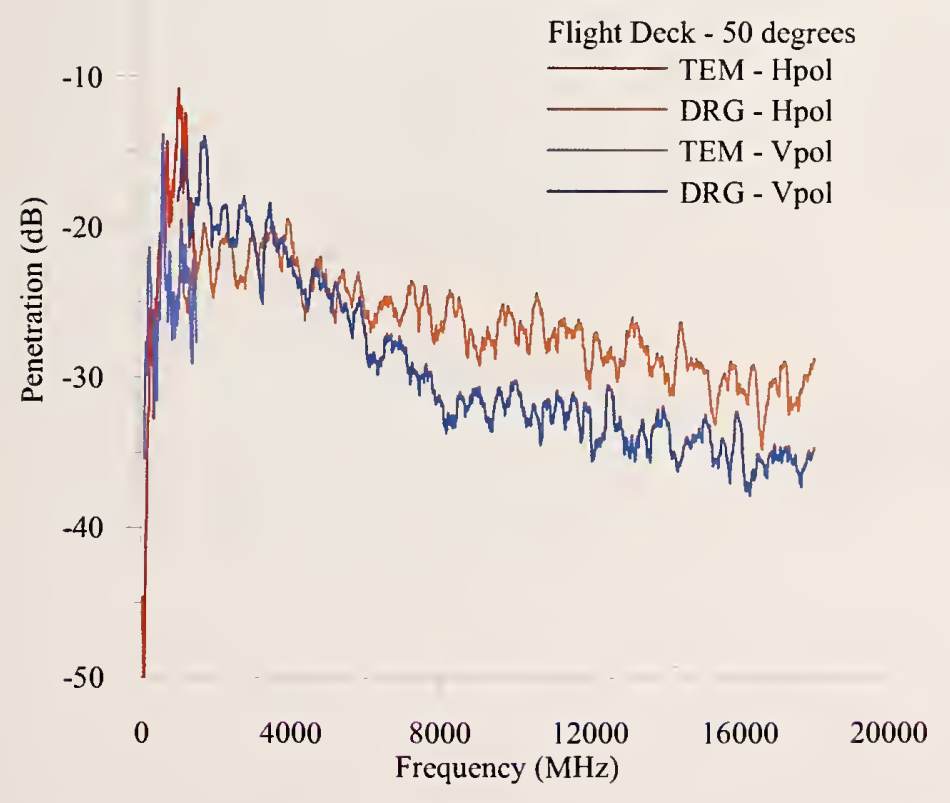

$330-30$

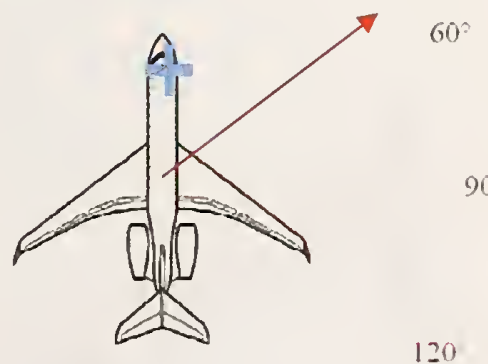

150

Figure 63. Penetration versus frequency for all antennas and polarizations for the transmitting tower placed at the $50^{\circ}$ position and the receiver placed in the flight deck. 
0

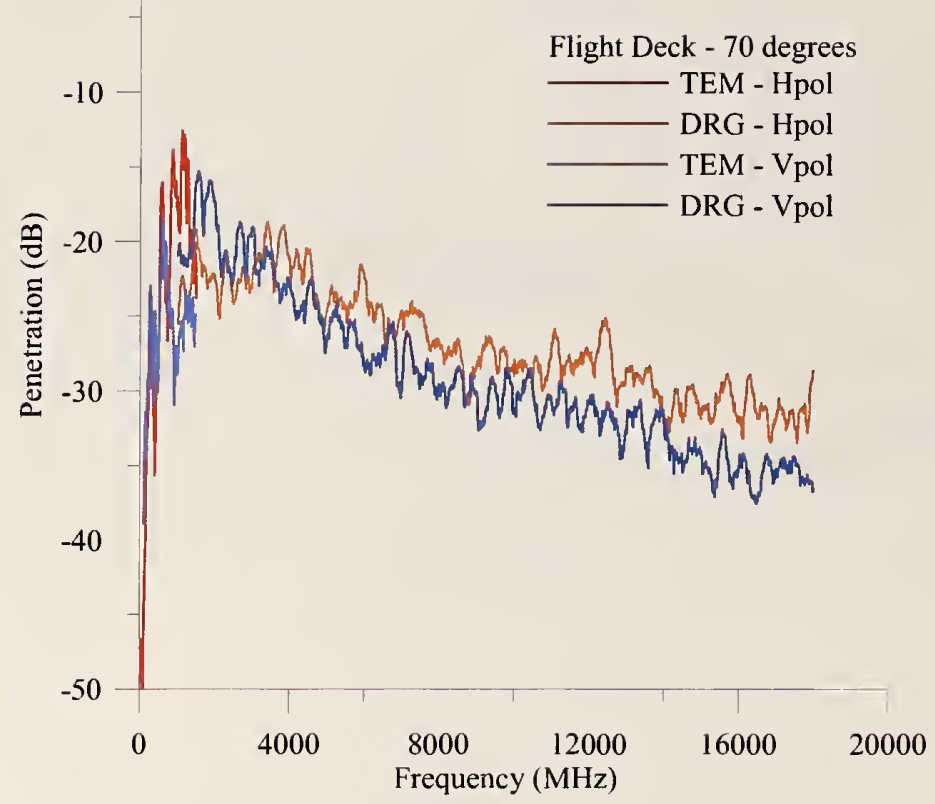

330

0 30

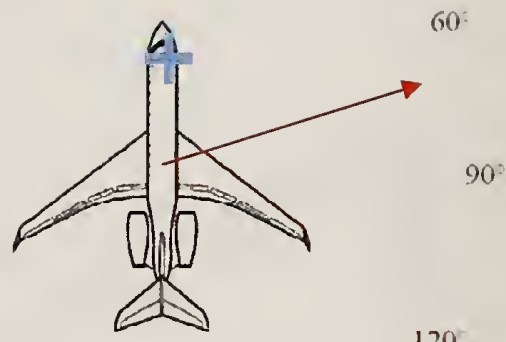

90

150

$180^{\circ}$

Figure 64. Penetration versus frequency for all antennas and polarizations for the transmitting tower placed at the $70^{\circ}$ position and the receiver placed in the flight deck.

0
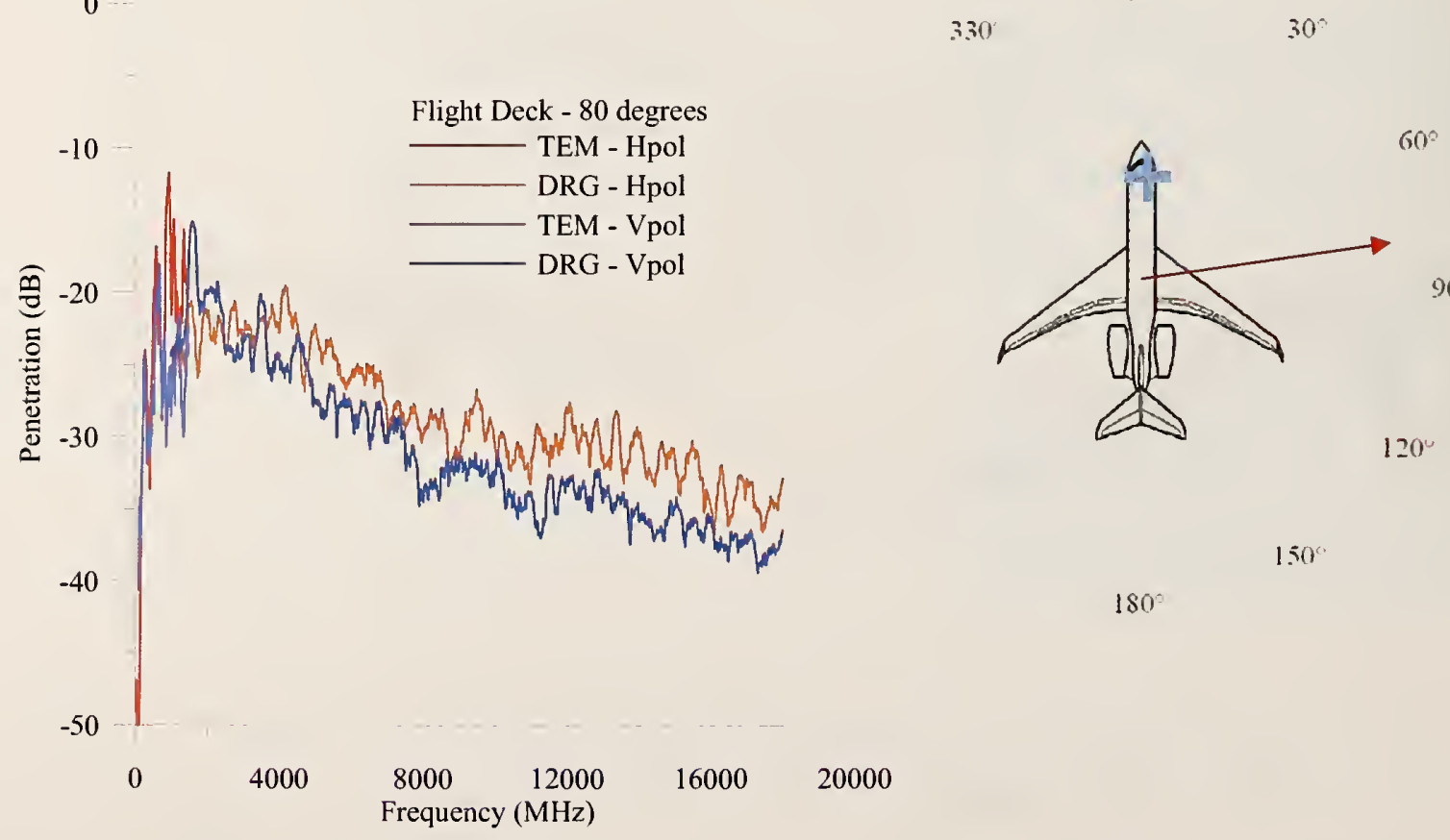

150

$180^{\circ}$

Figure 65. Penetration versus frequency for all antennas and polarizations for the transmitting tower placed at the $80^{\circ}$ position and the receiver placed in the flight deck. 


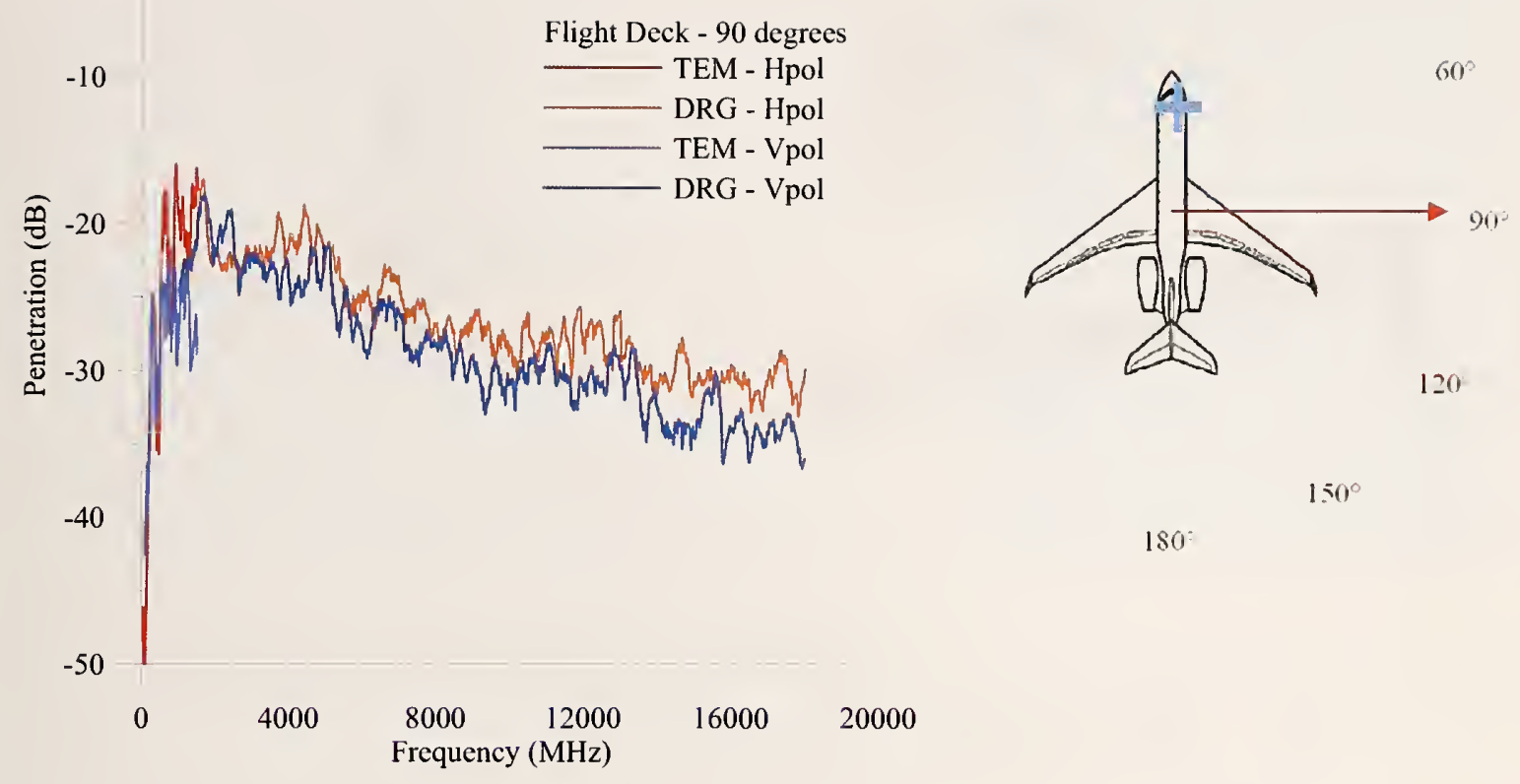

Figure 66. Penetration versus frequency for all antennas and polarizations for the transmitting tower placed at the $90^{\circ}$ position and the receiver placed in the flight deck.

0

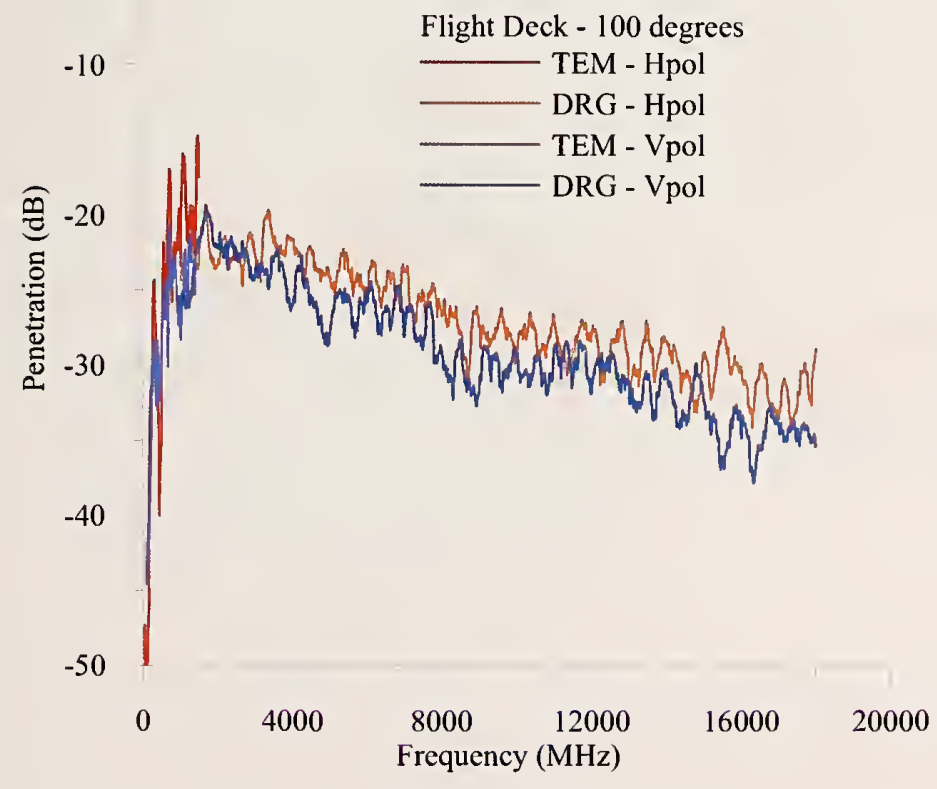

$330+30^{\circ}$

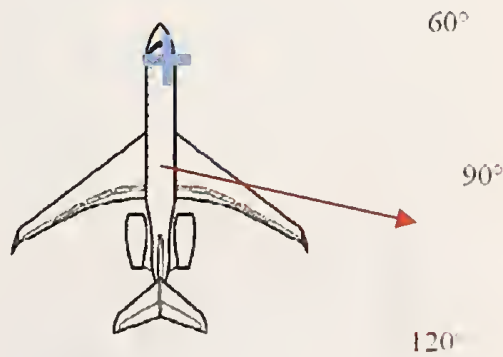

150

$180^{\circ}$

Figure 67. Penetration versus frequency for all antennas and polarizations for the transmitting tower placed at the $100^{\circ}$ position and the receiver placed in the flight deck. 

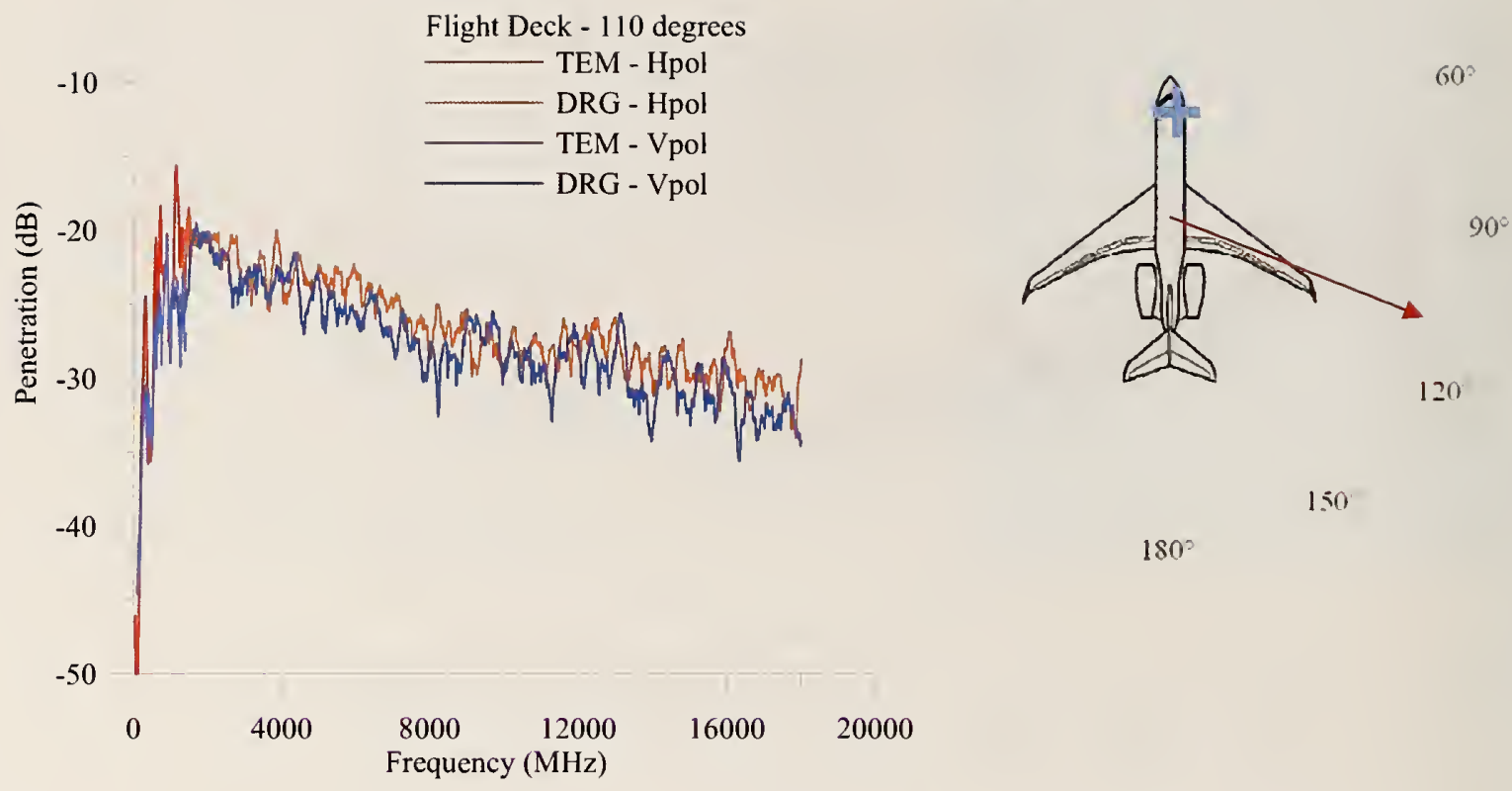

150

$180^{3}$

Figure 68. Penetration versus frequency for all antennas and polarizations for the transmitting tower placed at the $110^{\circ}$ position and the receiver placed in the flight deck.

0

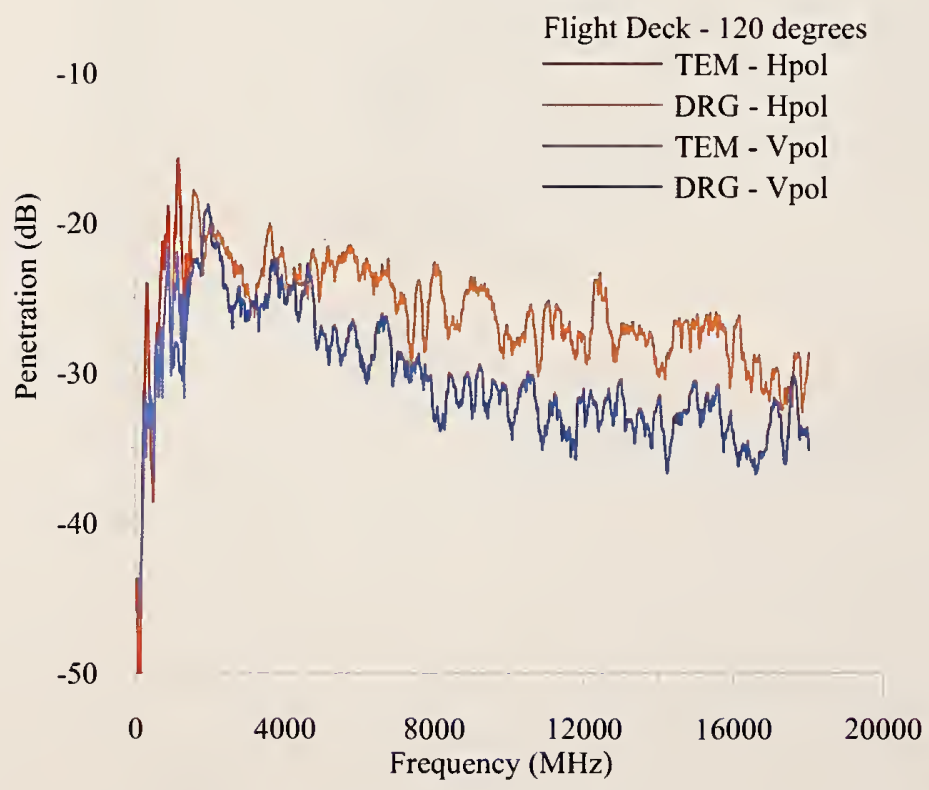

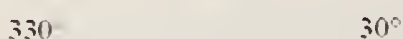

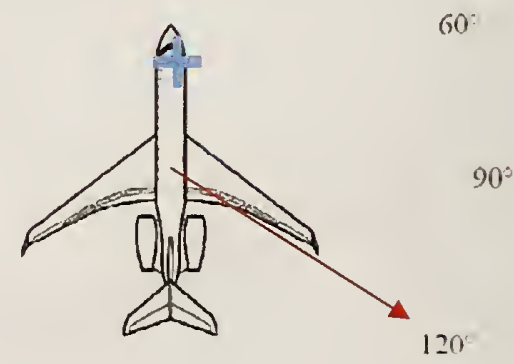

150

Figure 69. Penetration versus frequency for all antennas and polarizations for the transmitting tower placed at the $120^{\circ}$ position and the receiver placed in the flight deck. 


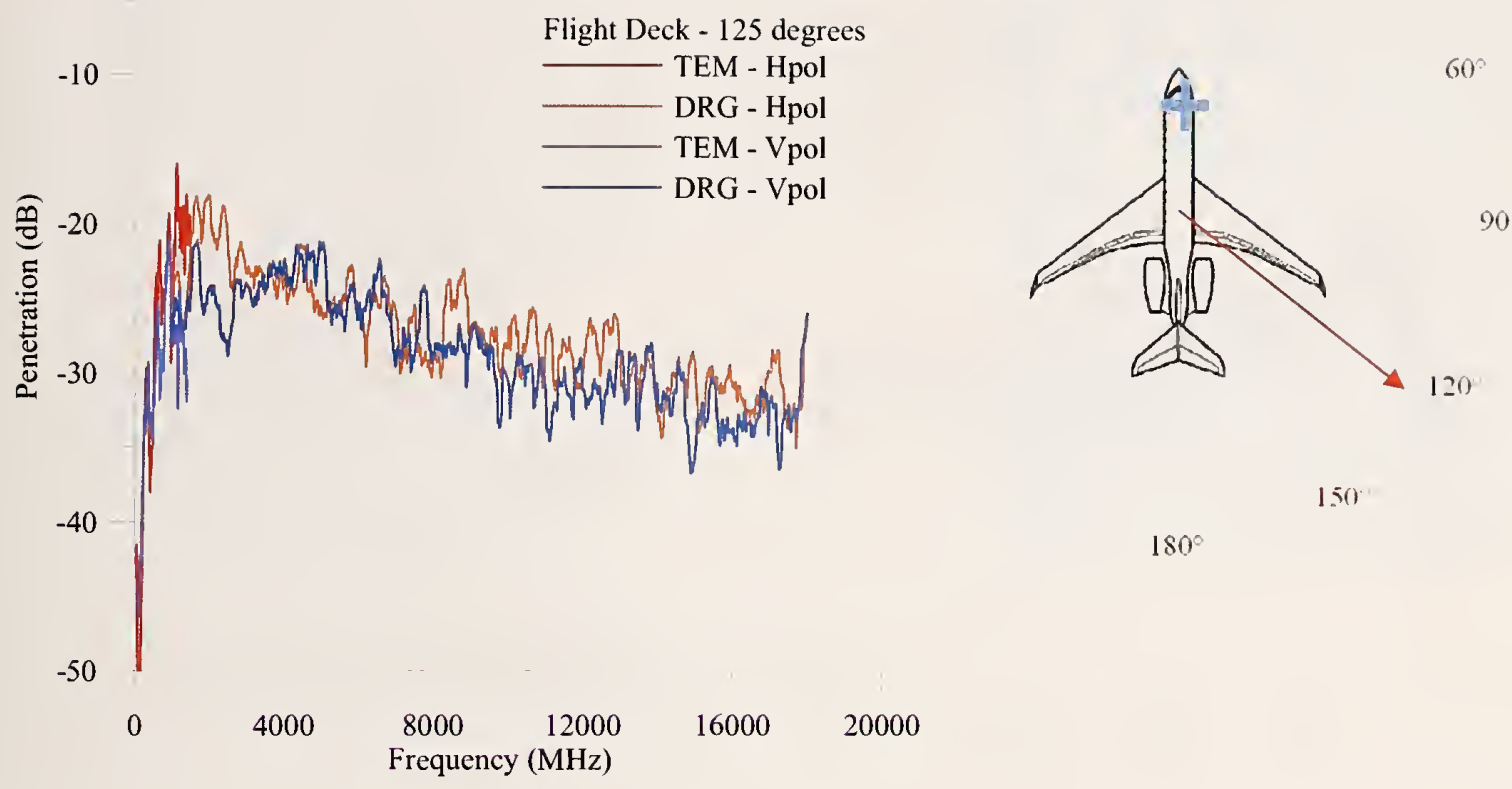

Figure 70. Penetration versus frequency for all antennas and polarizations for the transmitting tower placed at the $125^{\circ}$ position and the receiver placed in the flight deck.

0

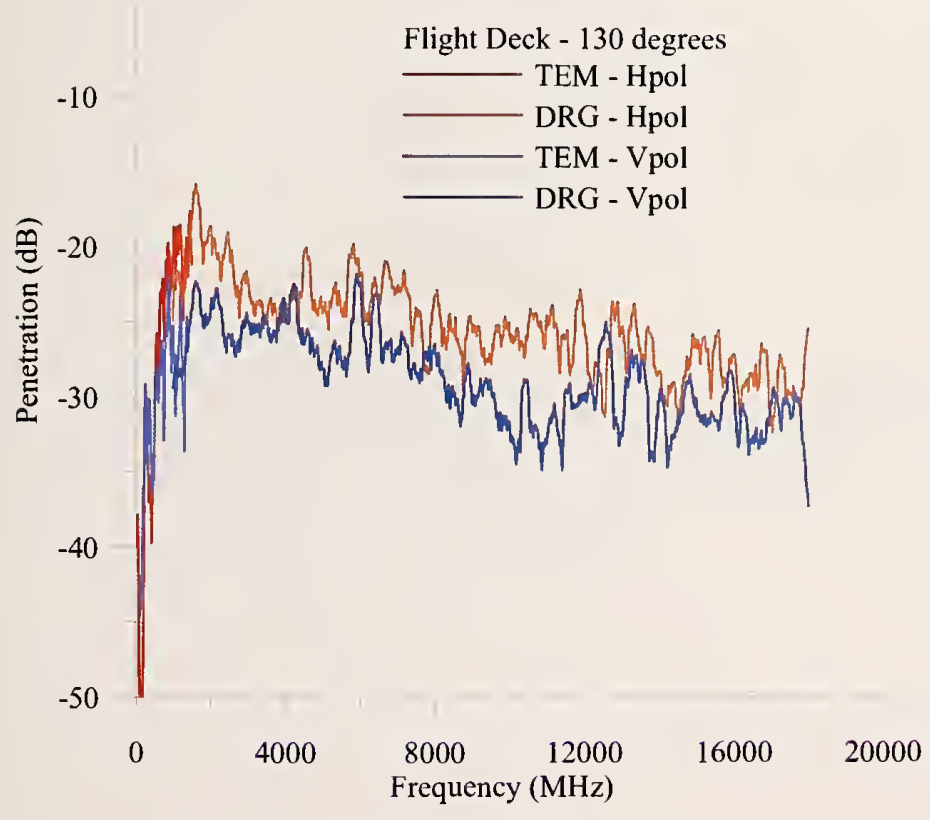

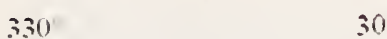

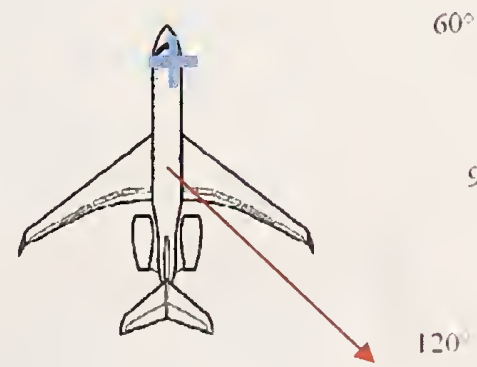

1.80

$180^{\circ}$

Figure 71. Penetration versus frequency for all antennas and polarizations for the transmitting tower placed at the $130^{\circ}$ position and the receiver placed in the flight deck. 


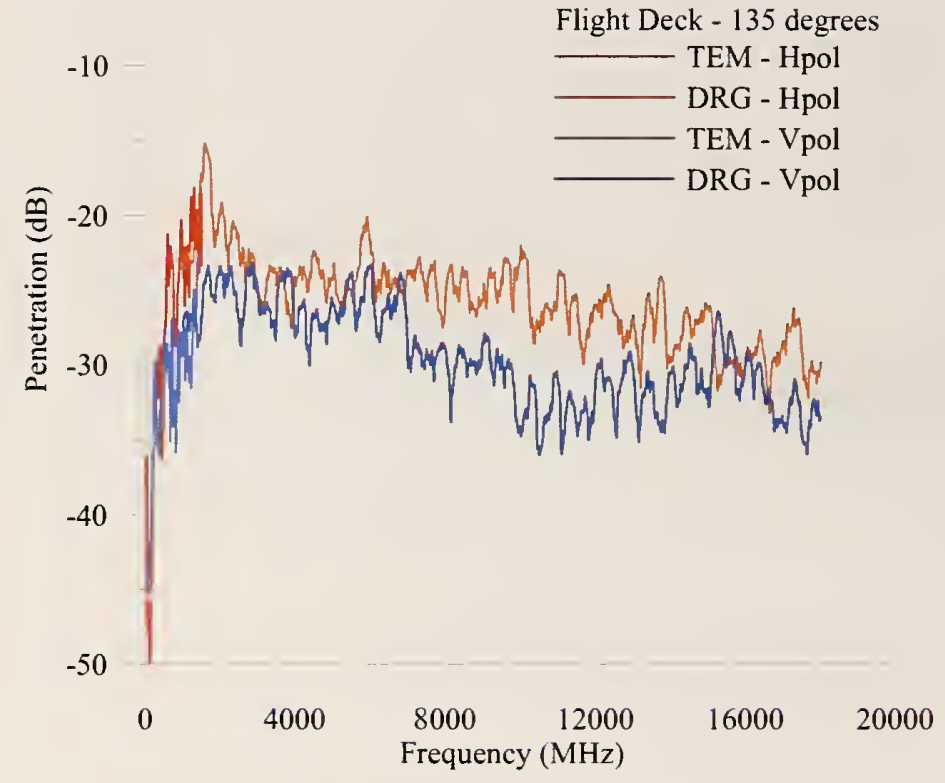

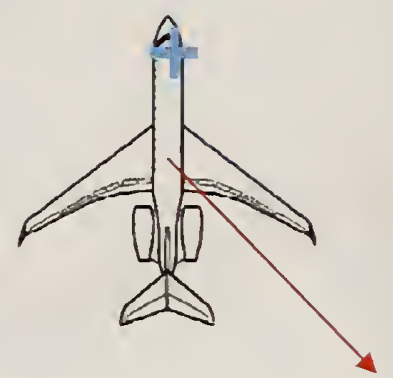

$60^{2}$

$90^{*}$

1.50

Figure 72. Penetration versus frequency for all antennas and polarizations for the transmitting tower placed at the $135^{\circ}$ position and the receiver placed in the flight deck.

0

330

Flight Deck - 140 degrees

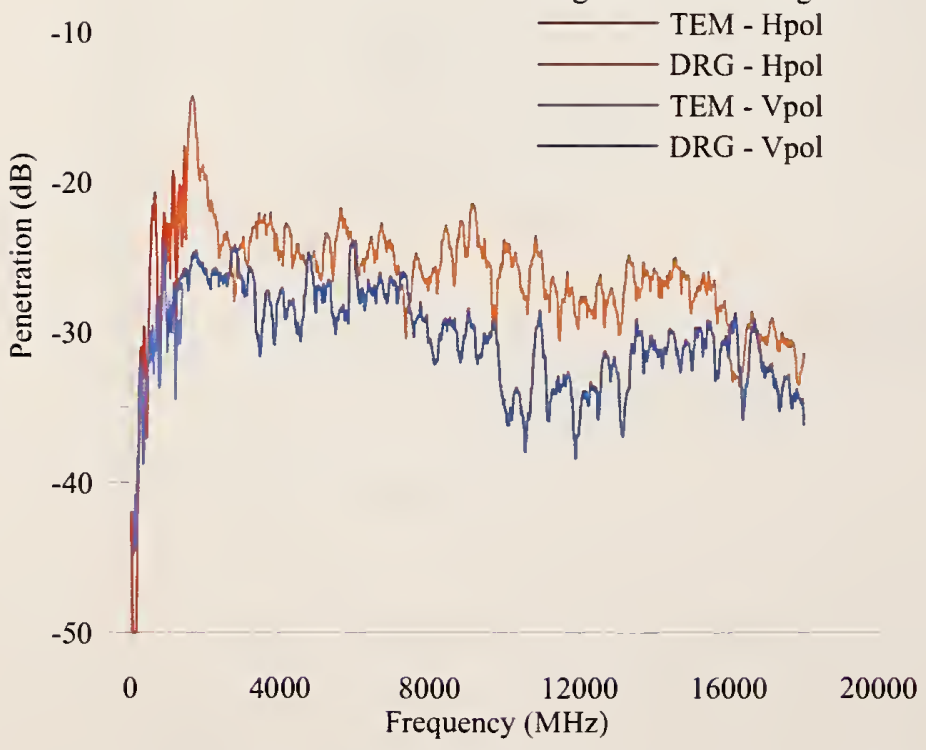

$60^{\circ}$

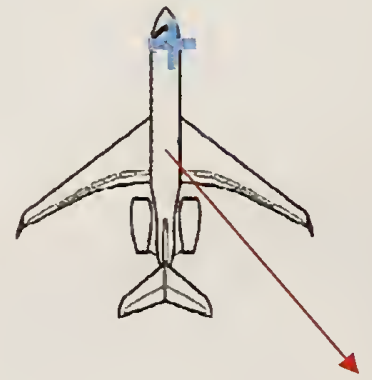

$90^{\circ}$

$120^{\prime}$

$150^{\circ}$

Figure 73. Penetration versus frequency for all antennas and polarizations for the transmitting tower placed at the $140^{\circ}$ position and the receiver placed in the flight deck. 
Flight Deck - 145 degrees
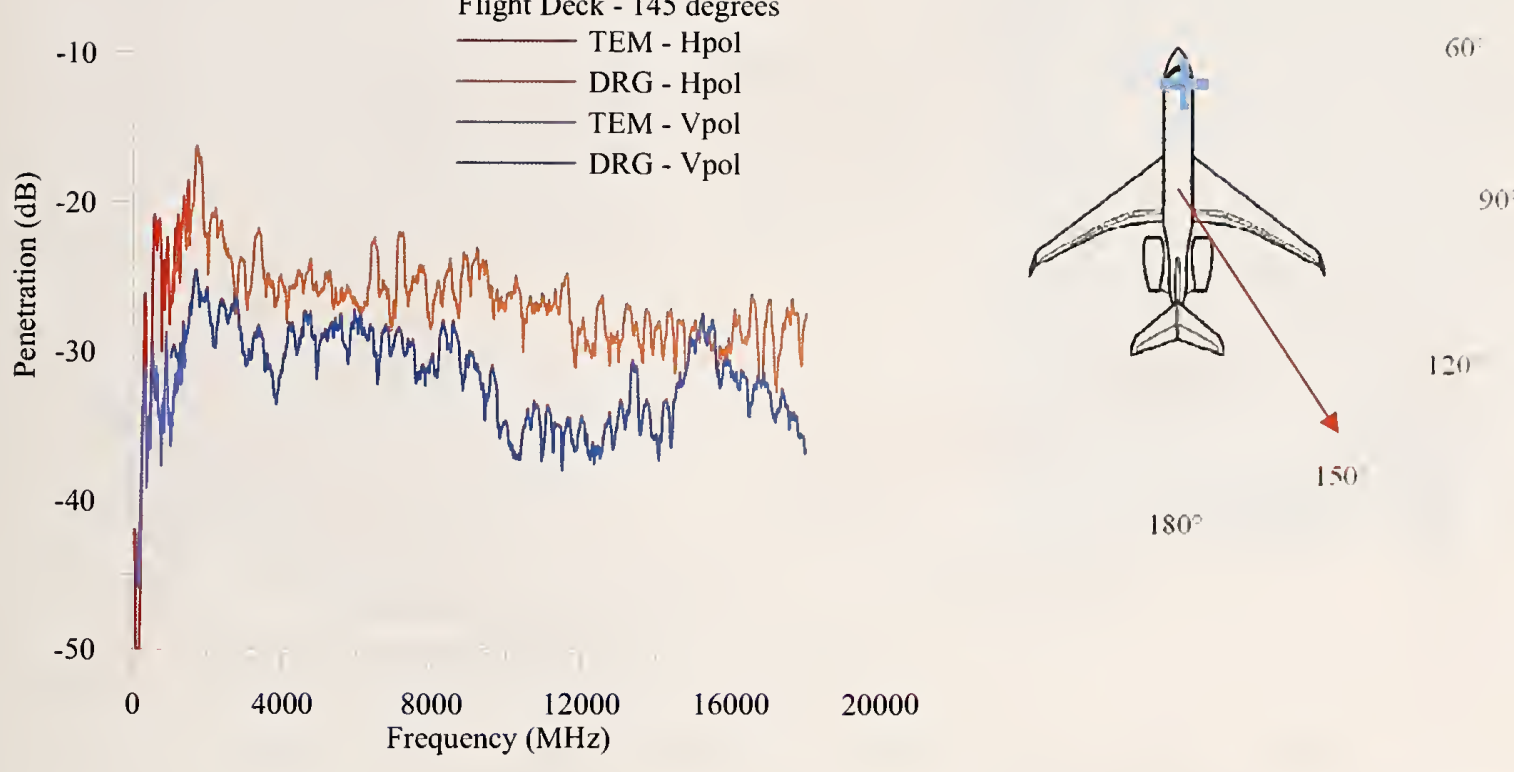

Figure 74. Penetration versus frequency for all antennas and polarizations for the transmitting tower placed at the $145^{\circ}$ position and the receiver placed in the flight deck.

0

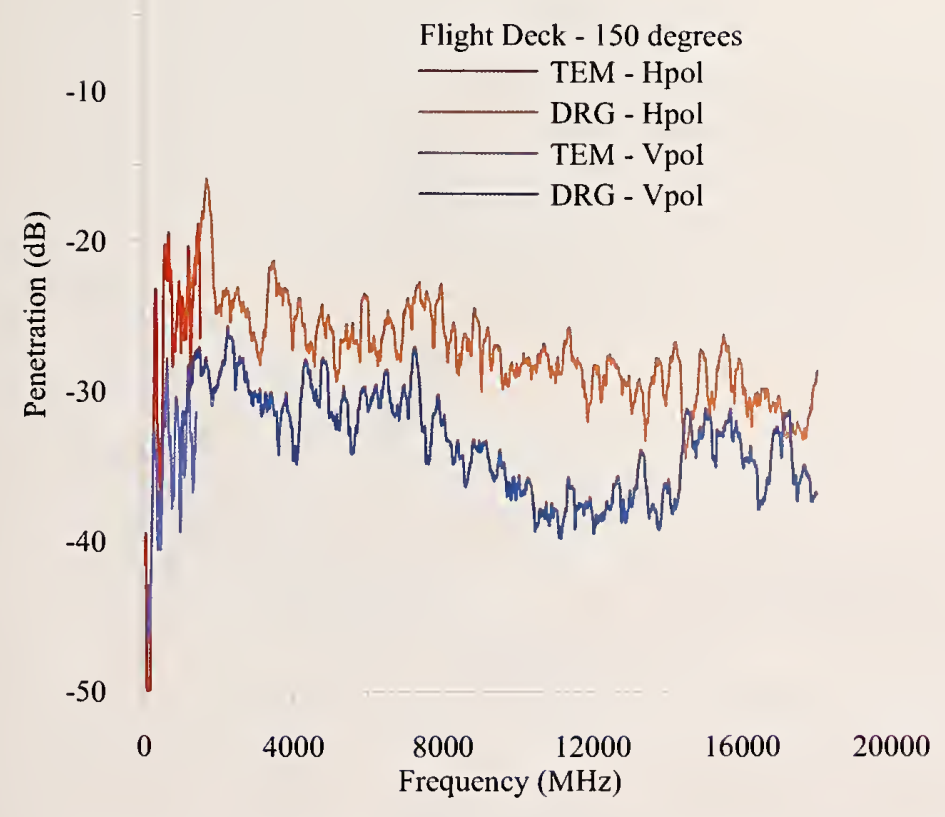

3.30

0

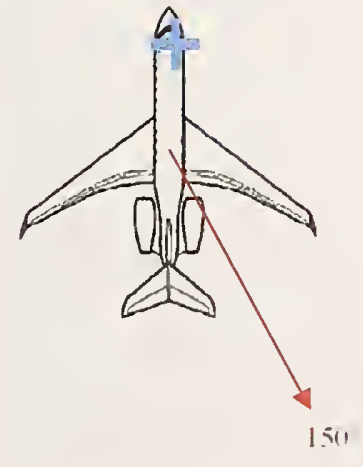

$60^{\circ}$

90

$180^{\circ}$

Figure 75. Penetration versus frequency for all antennas and polarizations for the transmitting tower placed at the $150^{\circ}$ position and the receiver placed in the flight deck. 


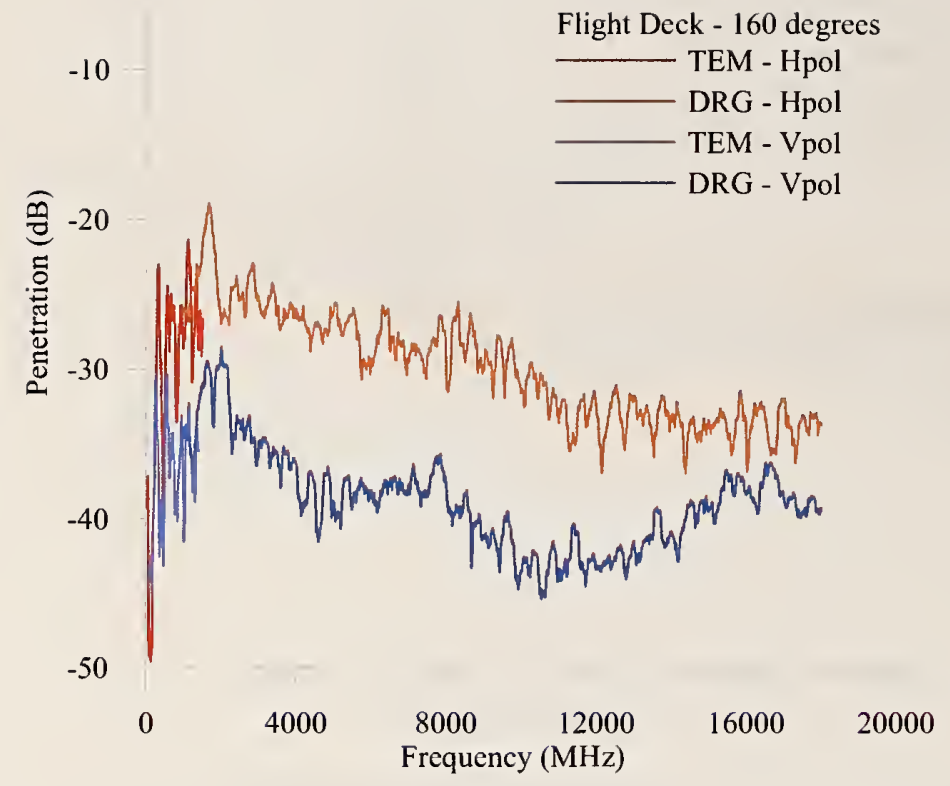

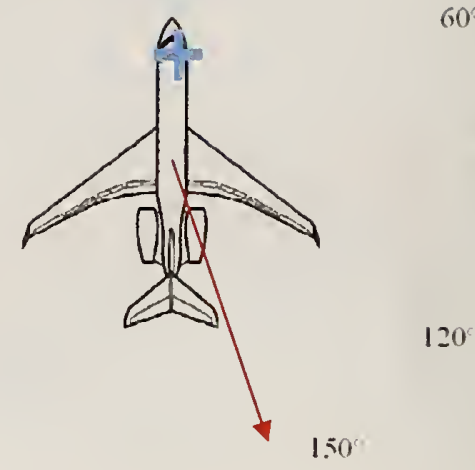

$180^{\circ}$

Figure 76. Penetration versus frequency for all antennas and polarizations for the transmitting tower placed at the $160^{\circ}$ position and the receiver placed in the flight deck.

0

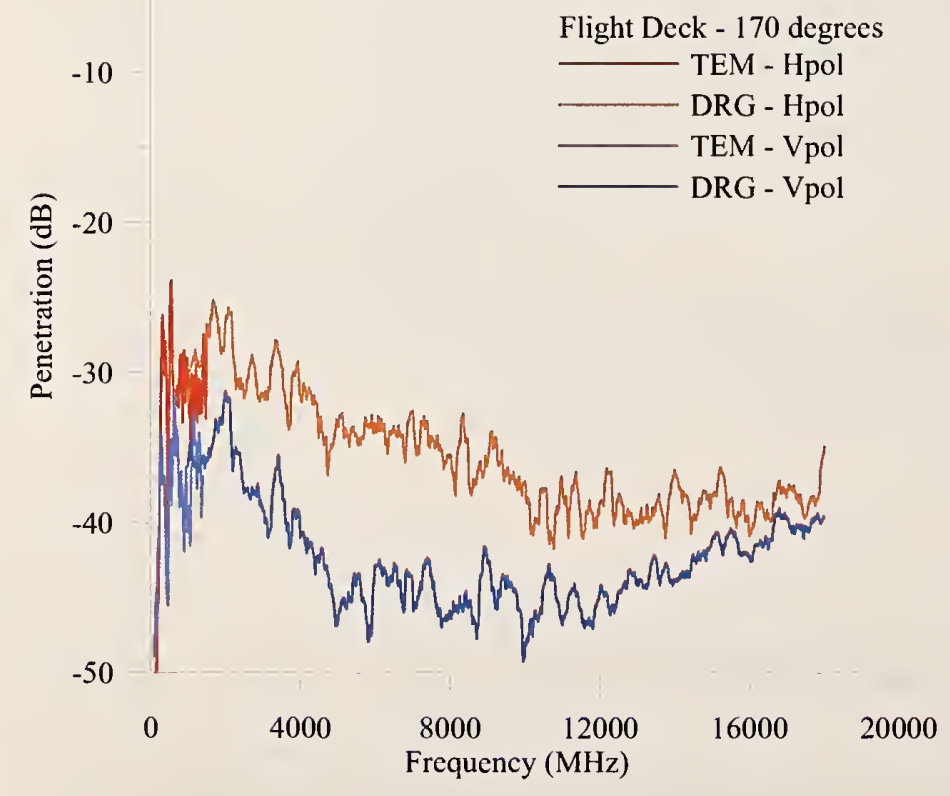

330

0

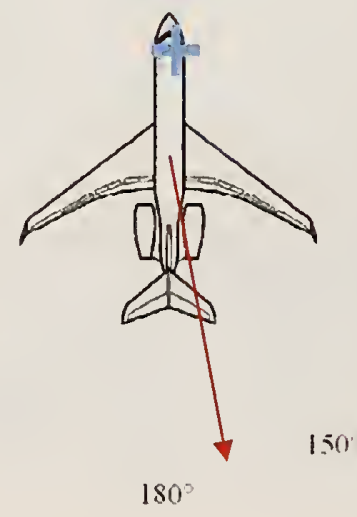

$60^{\circ}$

$90^{\circ}$

$120^{\circ}$ 

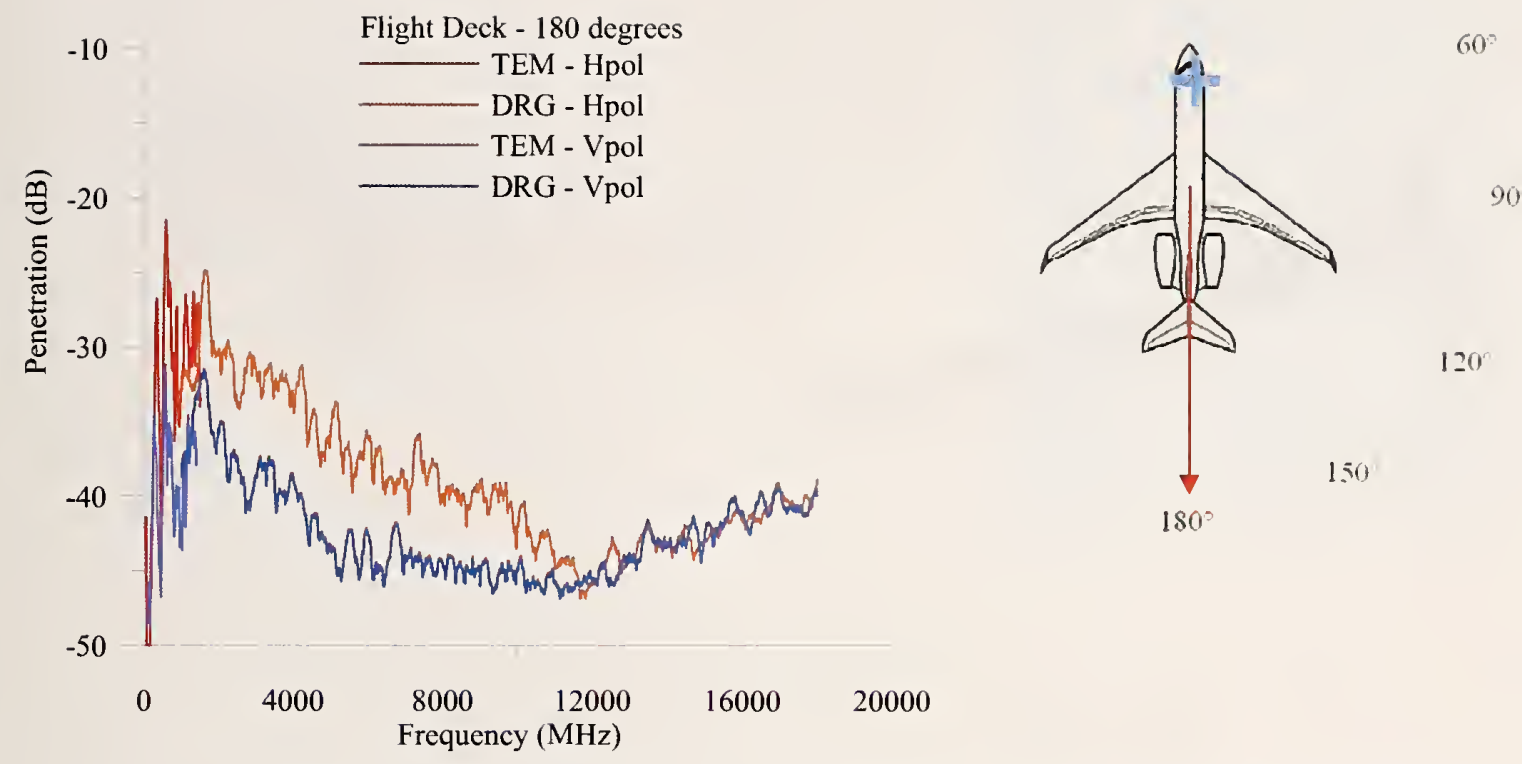

Figure 78. Penetration versus frequency for all antennas and polarizations for the transmitting tower placed at the $180^{\circ}$ position and the receiver placed in the flight deck.

0

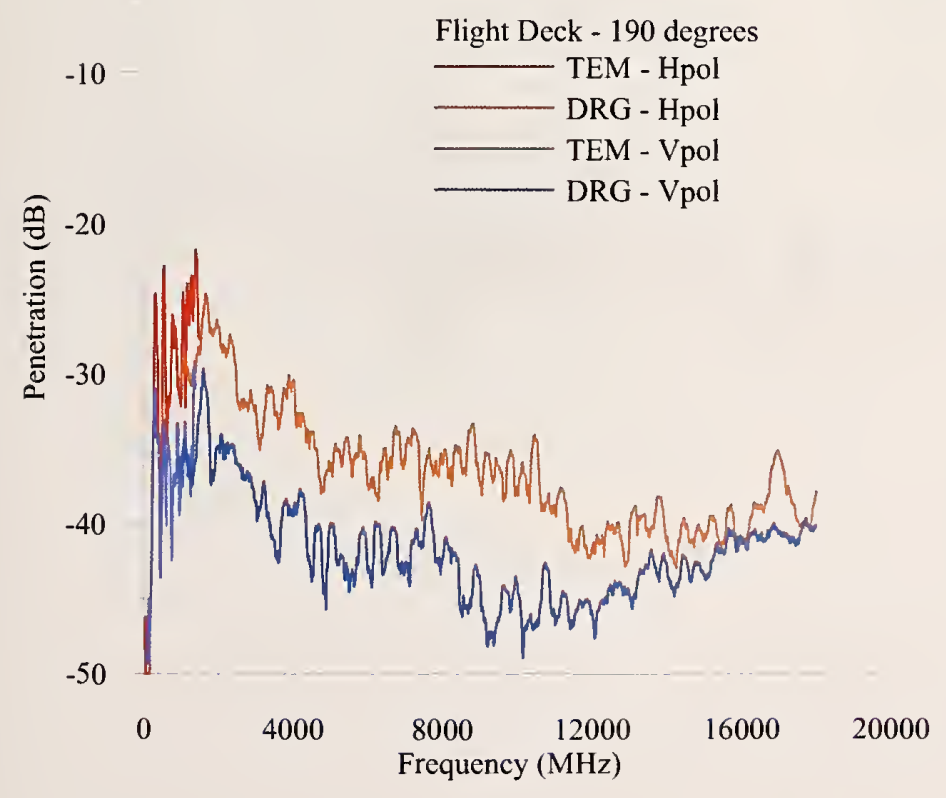

330$$
0
$$$$
30^{\circ}
$$

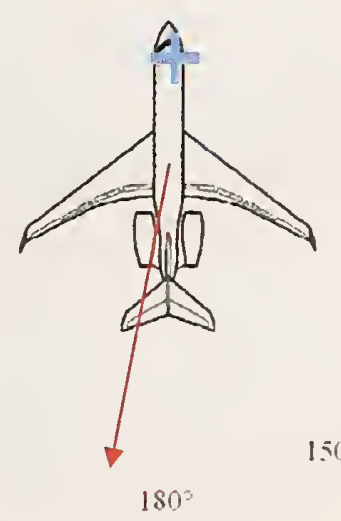

$60^{\circ}$

Figure 79. Penetration versus frequency for all antennas and polarizations for the transmitting tower placed at the $190^{\circ}$ position and the receiver placed in the flight deck. 
0

330

$30^{\circ}$

Flight Deck - 270 degrecs
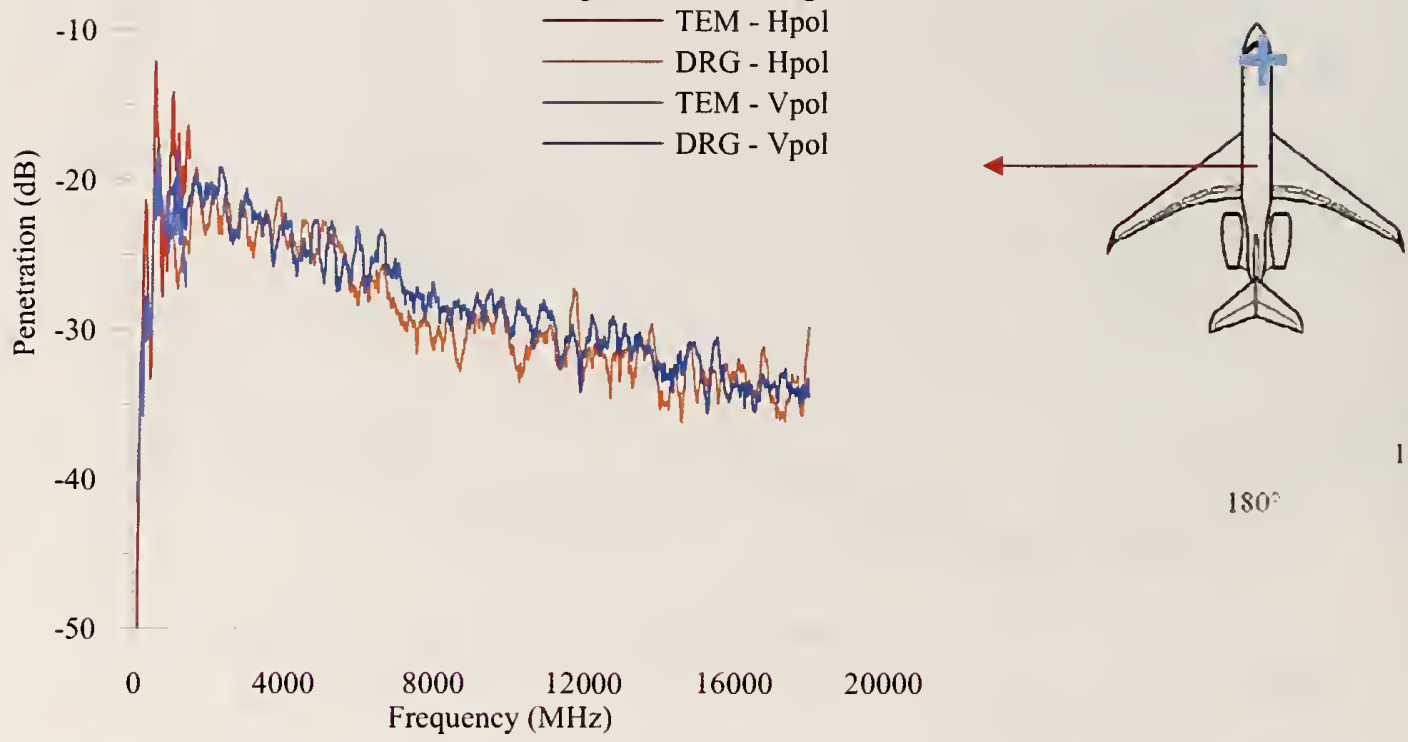

Figure 80. Penetration versus frequency for all antennas and polarizations for the transmitting tower placed at the $270^{\circ}$ position and the receiver placed in the flight deck.
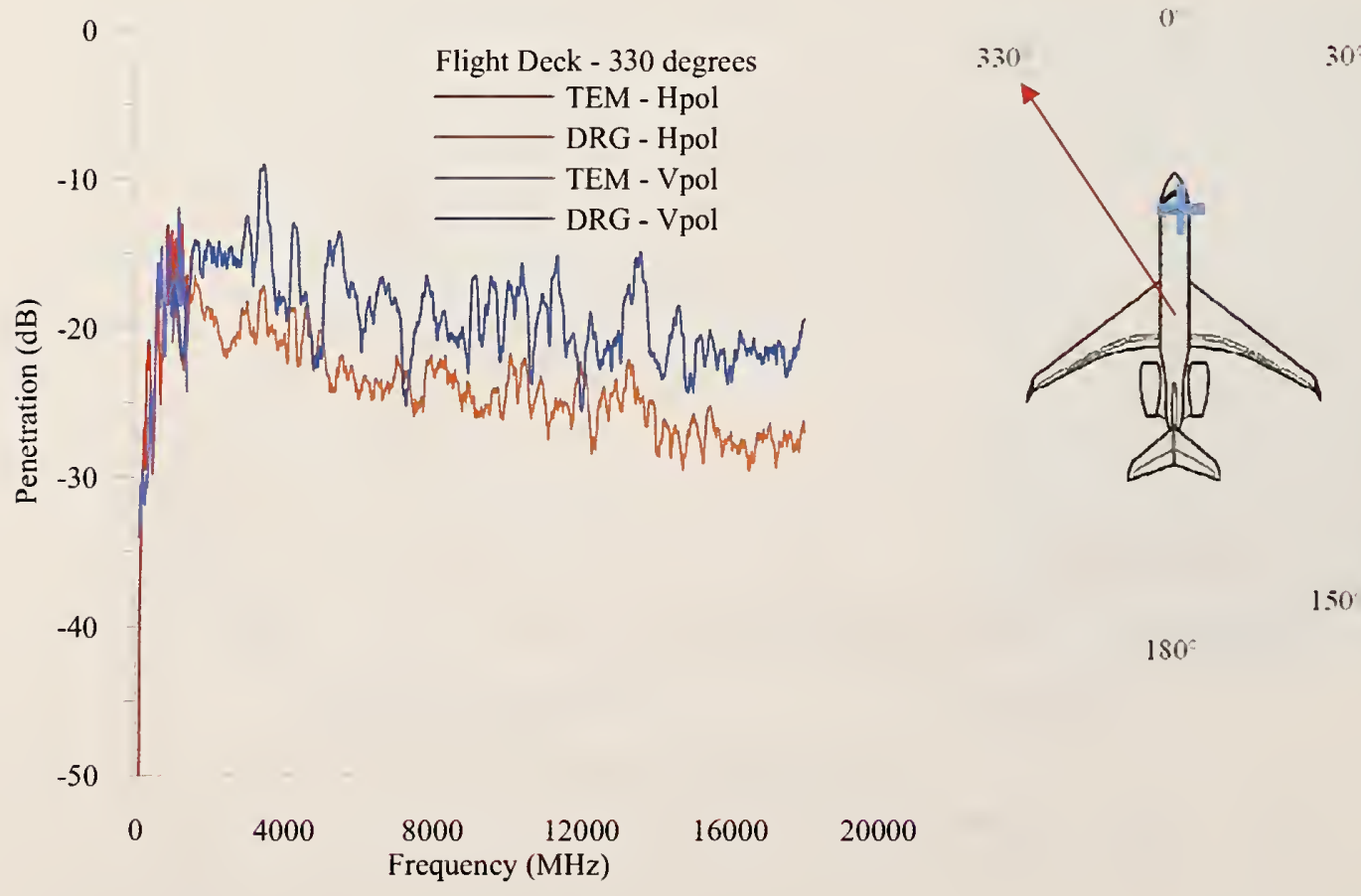

$60^{\circ}$

$90^{3}$

120

150

180

Figure 81. Penetration versus frequency for all antennas and polarizations for the transmitting tower placed at the $330^{\circ}$ position and the receiver placed in the flight deck. 


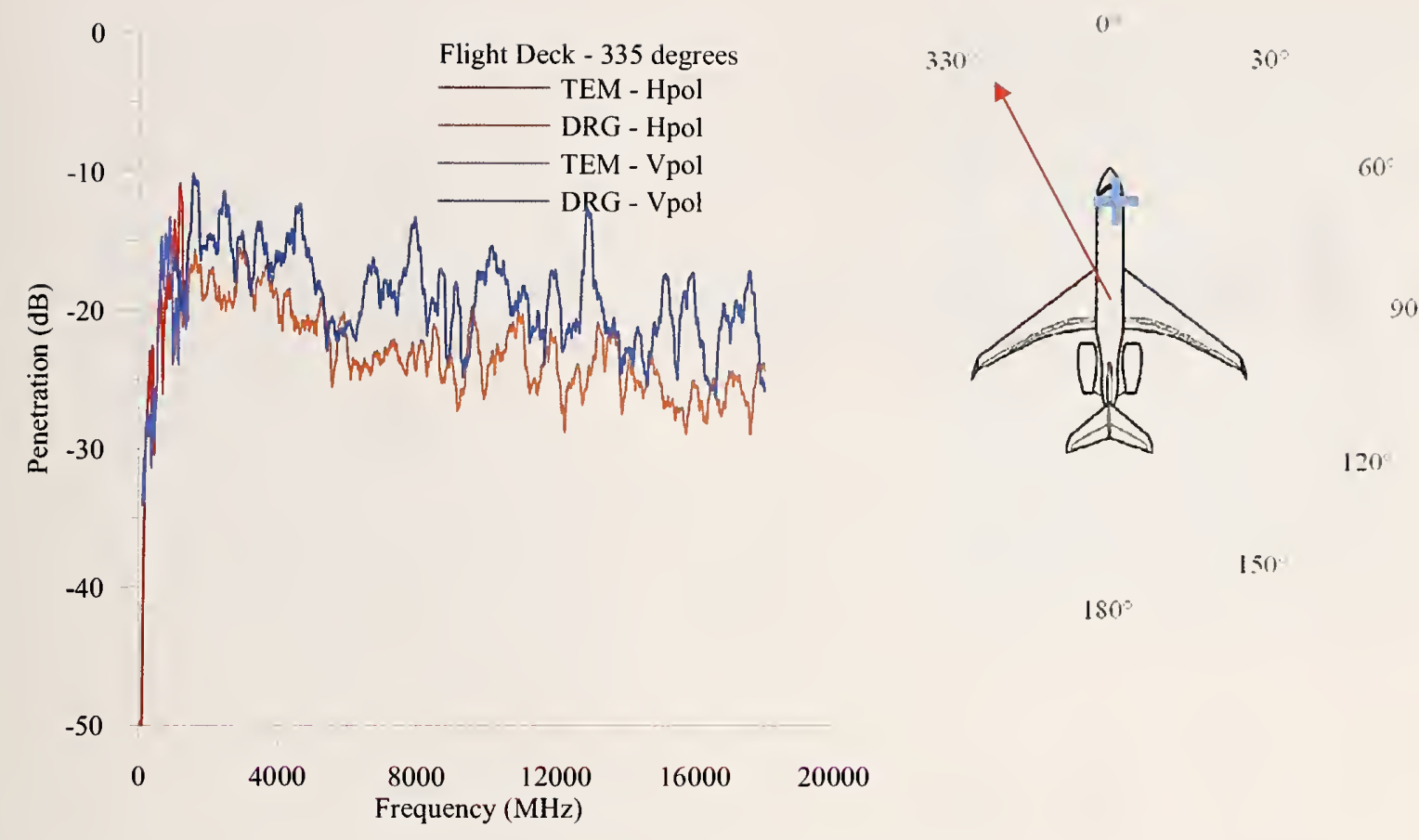

Figure 82. Penetration versus frequency for all antennas and polarizations for the transmitting tower placed at the $335^{\circ}$ position and the receiver placed in the flight deck.
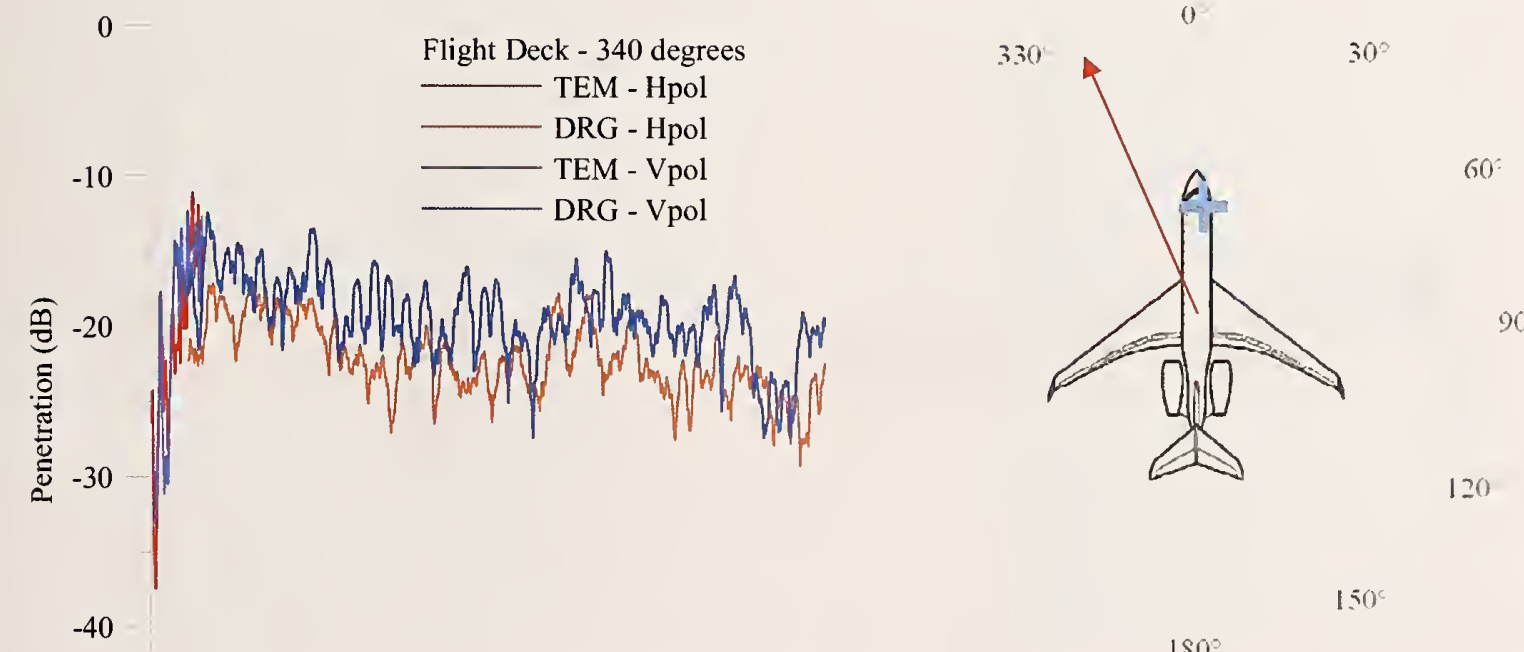

$180^{\circ}$

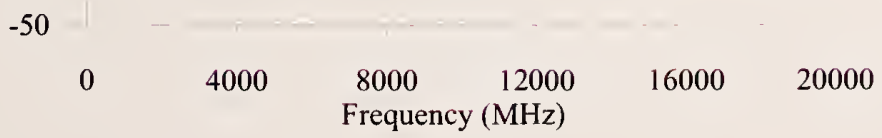

Figure 83. Penetration versus frequency for all antennas and polarizations for the transmitting tower placed at the $340^{\circ}$ position and the receiver placed in the flight deck. 

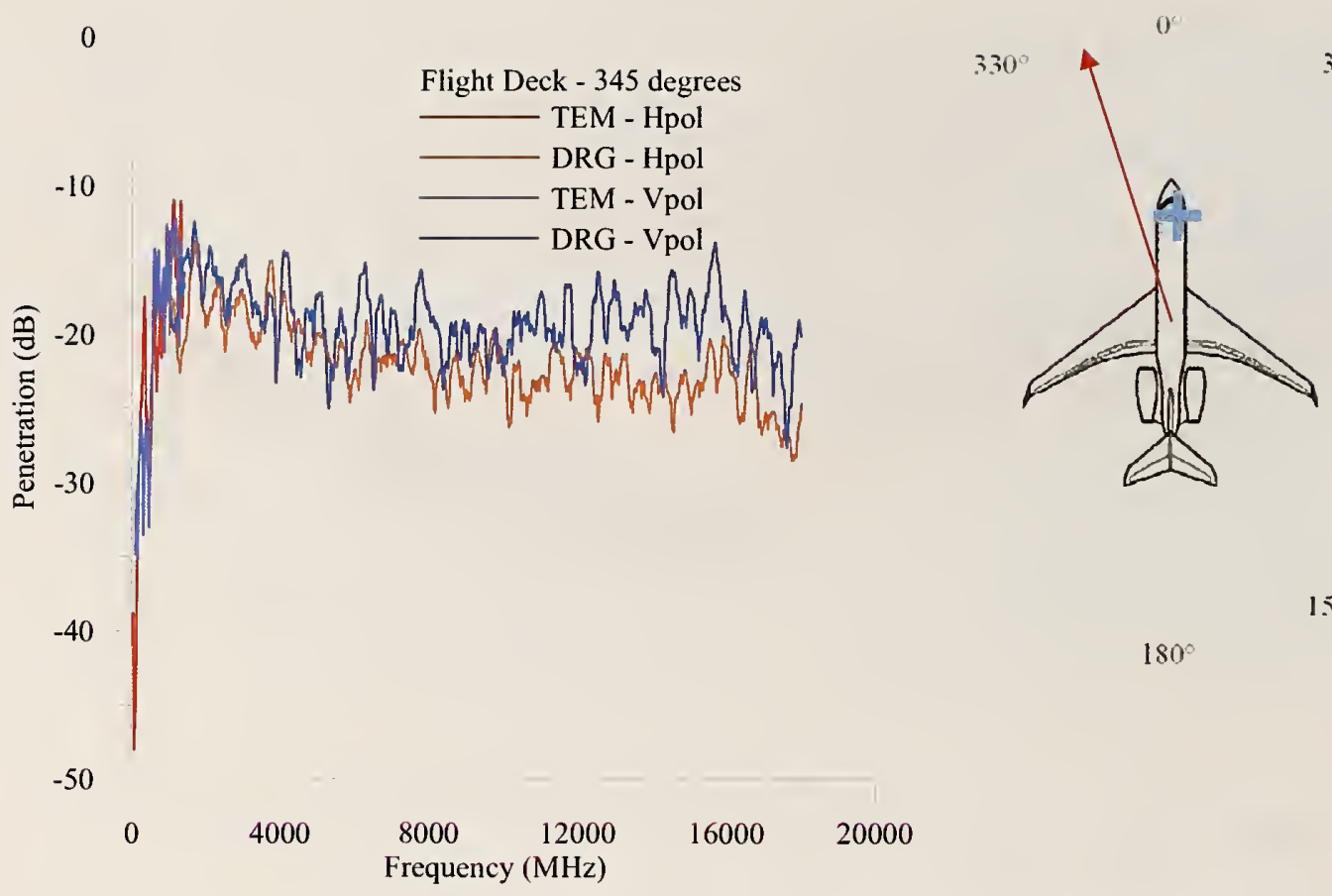

$60^{\circ}$

$90^{\circ}$

$120^{\circ}$

150

$180^{\circ}$

Figure 84. Penetration versus frequency for all antennas and polarizations for the transmitting tower placed at the $345^{\circ}$ position and the receiver placed in the flight deck.
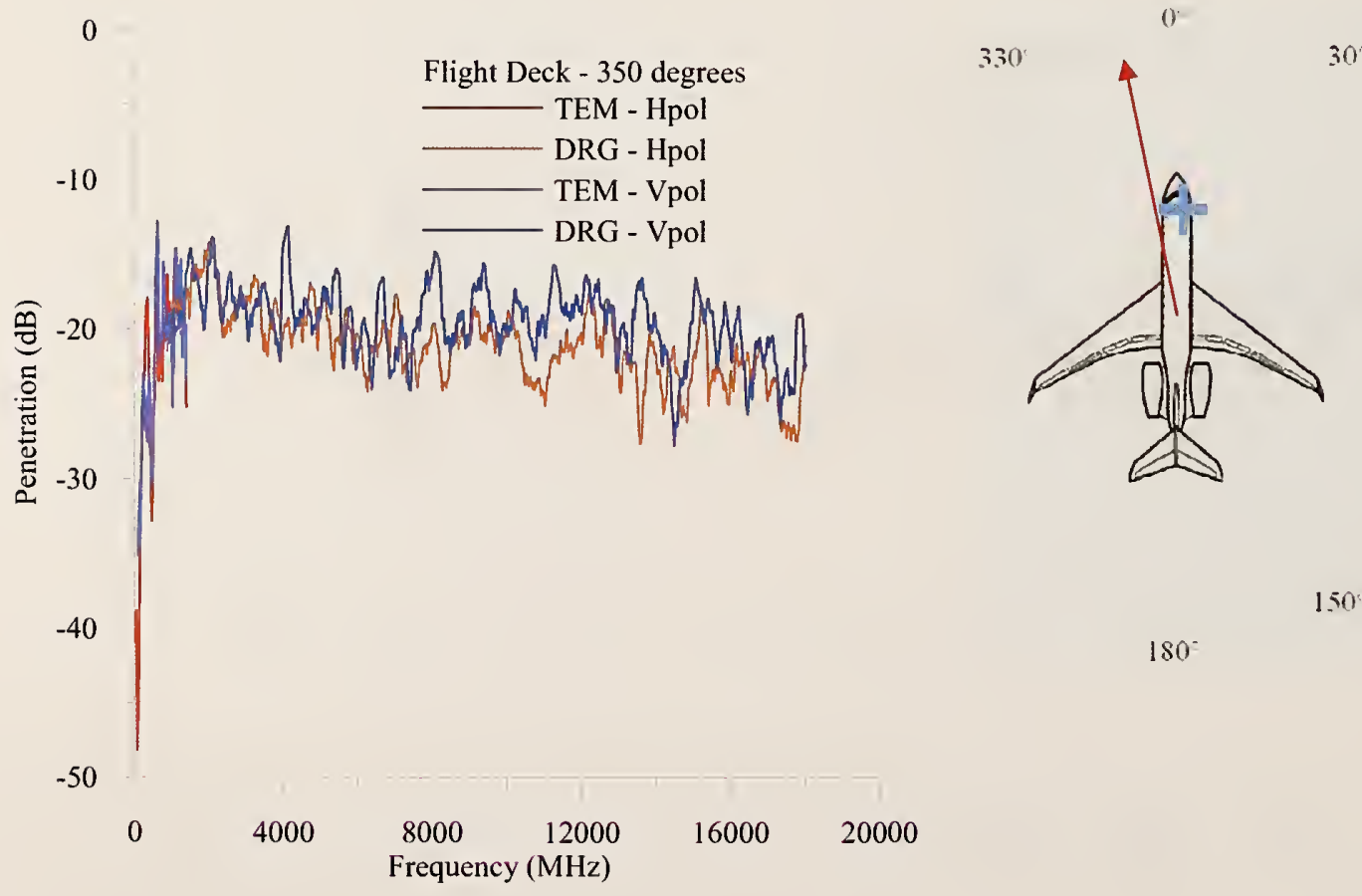

$60^{\circ}$

$90^{\circ}$

120

$150^{\circ}$

$180^{\circ}$

Figure 85. Penetration versus frequency for all antennas and polarizations for the transmitting tower placed at the $350^{\circ}$ position and the receiver placed in the flight deck. 


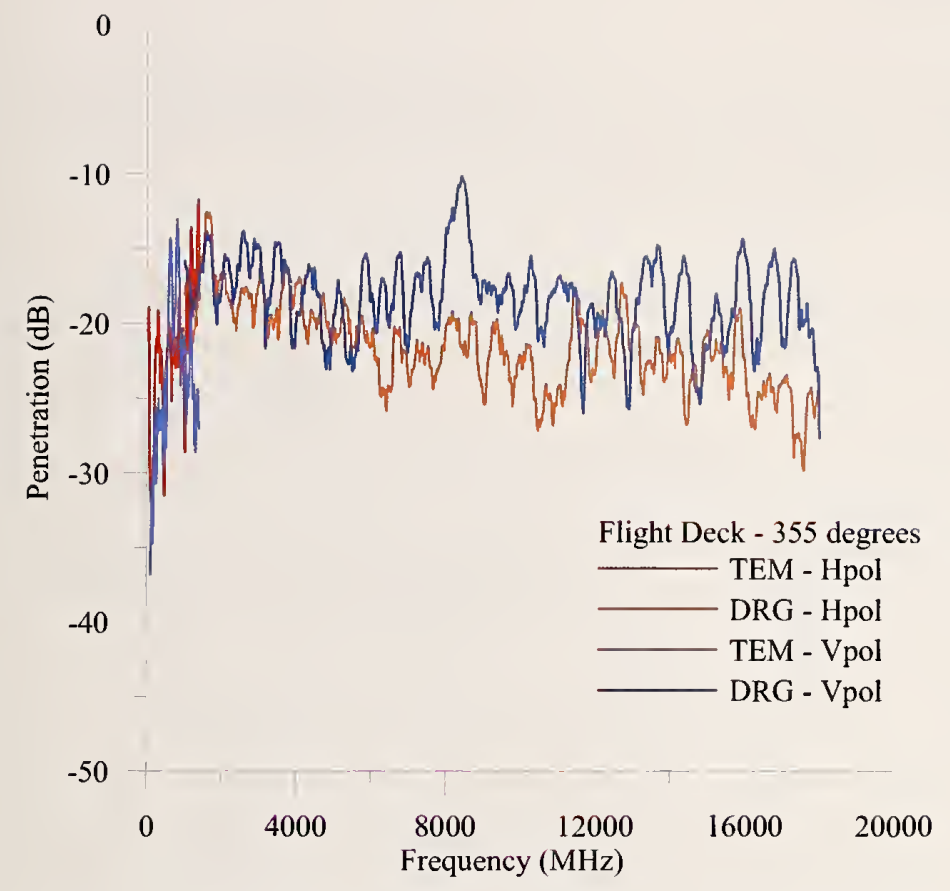

Figure 86. Penetration versus frequency for all antennas and polarizations for the transmitting tower placed at the $355^{\circ}$ position and the receiver placed in the flight deck. 


\subsection{Cargo Closet Penetration Results}

A series of penetration measurements were taken with the receiving antennas placed in the cargo closet located at the rear of the main passenger cabin. The receiving antenna positions for the TEM horn antennas and DRG antennas are shown in Figure 87.

Figure 88 shows the time-domain plots for the DRG antenna in the horizontal polarization for various positions around the aircraft. We see no direct antenna-to-antenna coupling in these plots due to the lack of any direct coupling paths from the outside of the aircraft into the cargo closet. Because there is no hardened door for this compartment we do expect to see more penetration than for typical cargo bays found on commercial aircraft. The frequency-domain plots corresponding to these same time-domain plots are shown in Figure 89. A pronounced resonance peak in penetration is seen in the neighborhood of $600 \mathrm{MHz}$ for vertically polarized antenna positions of the TEM horn antenna for the $60^{\circ}$ position to the $180^{\circ}$ position. The horizontally polarized antennas generally show greater penetration as we move around the aircraft. Maximum penetration of around $-15 \mathrm{~dB}$ occurs at the $30^{\circ}, 60^{\circ}, 330^{\circ}$, and $350^{\circ}$ positions at the lower frequencies, and approximately $-20 \mathrm{~dB}$ at these same positions at the higher frequencies. Minimum penetration occurs for the vertically polarized antennas at the $0^{\circ}$, $180^{\circ}$, and $350^{\circ}$ positions of approximately $-30 \mathrm{~dB}$ for the lower frequencies, and approximately $-45 \mathrm{~dB}$ at the middle frequencies. Symmetry plots are shown in Figure 90. Penetration tends to be symmetrical with the receiving antenna in the cargo closet, although the vertical polarization has approximately $5 \mathrm{~dB}$ less penetration than horizontal polarization. Detailed penetration data for every external angle are shown in Figures 91 to 120.

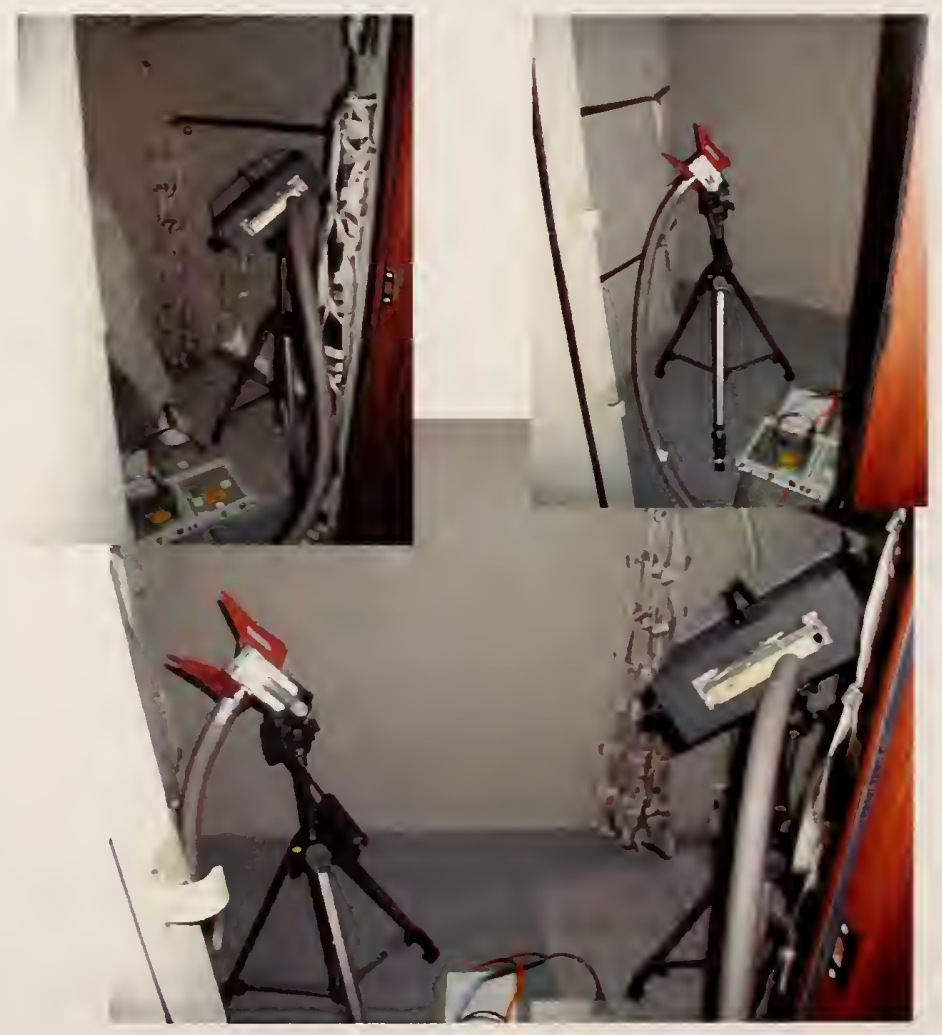

Figure 87. TEM and DRG antenna placement in the cargo closet. 


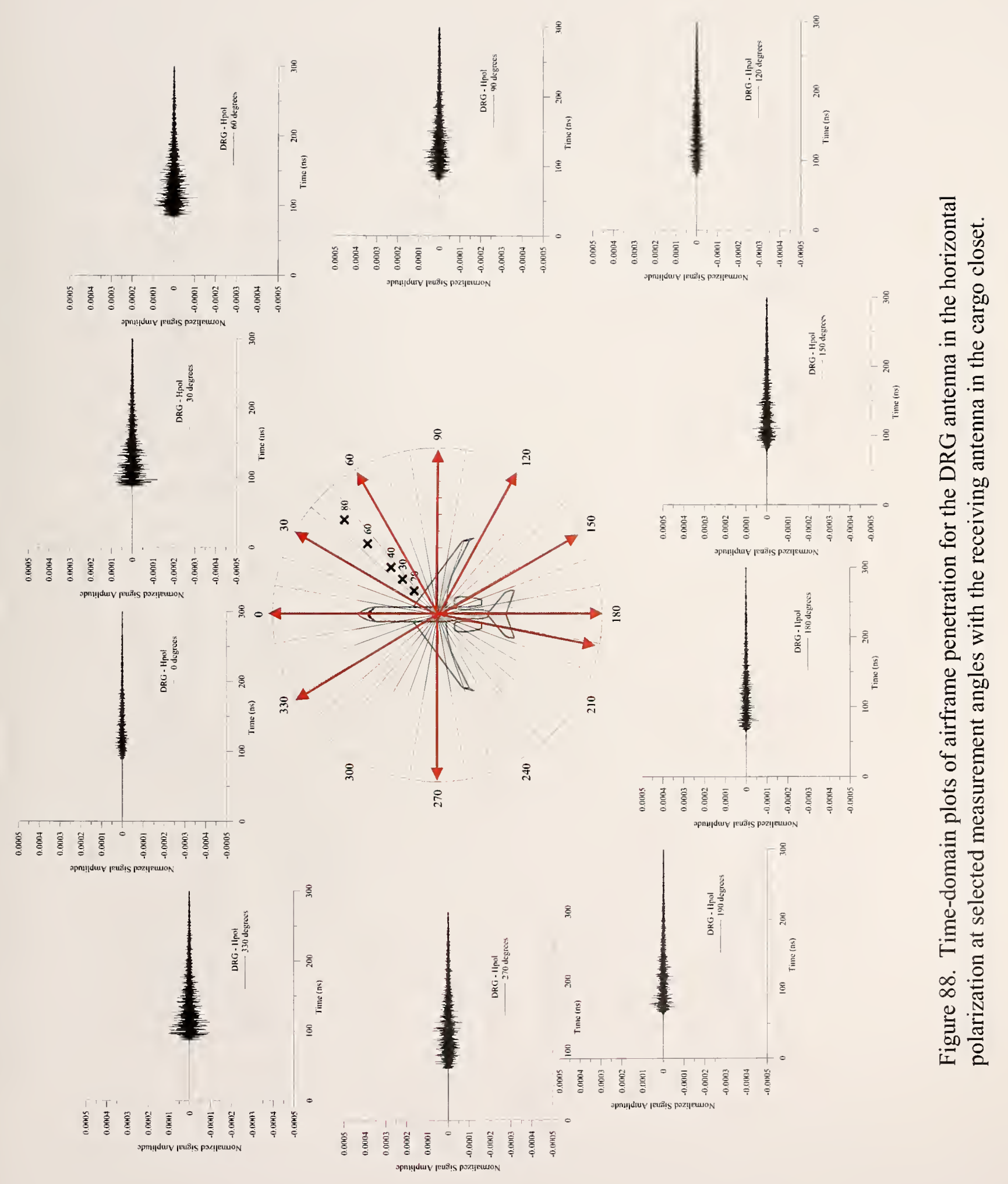




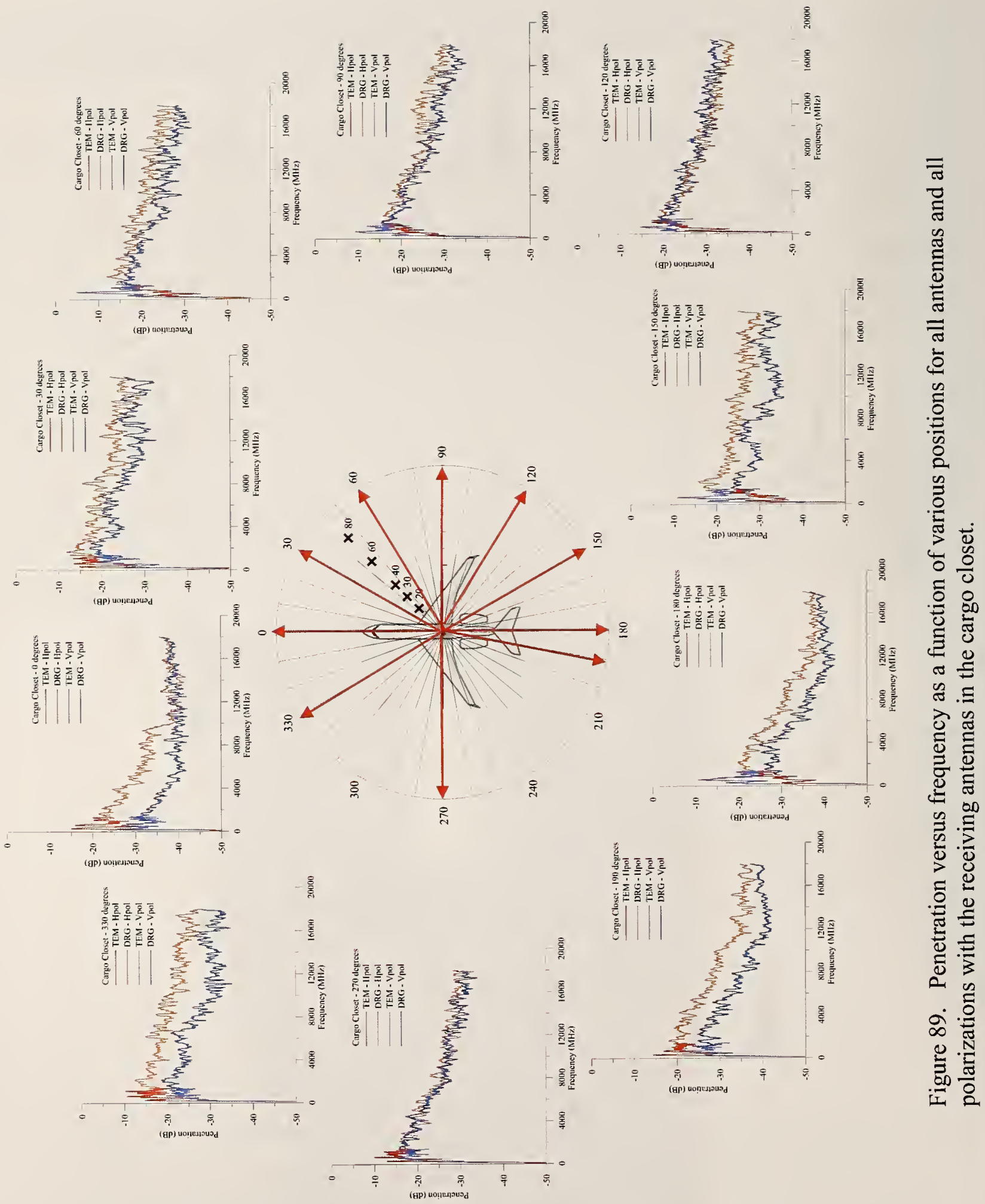




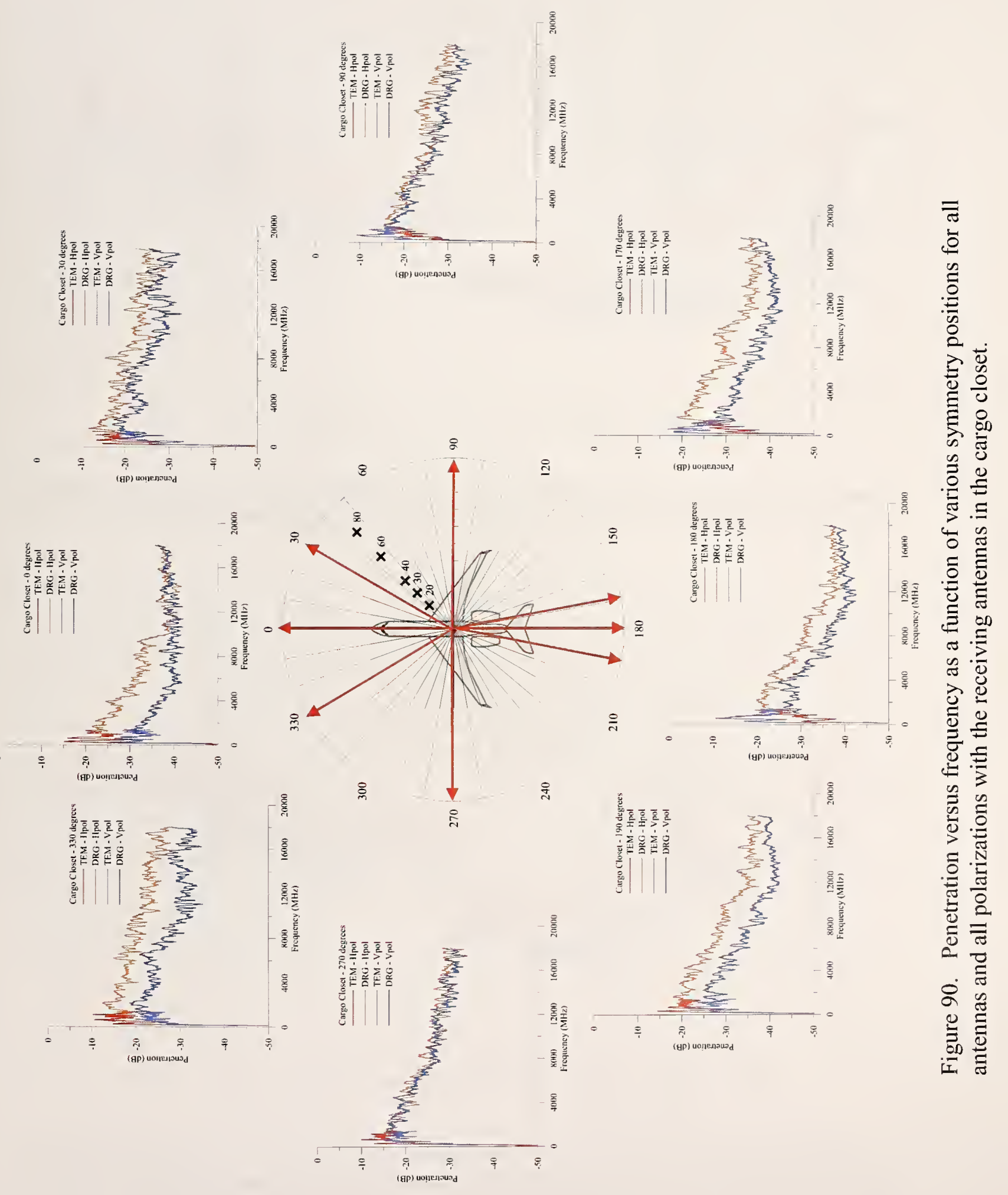




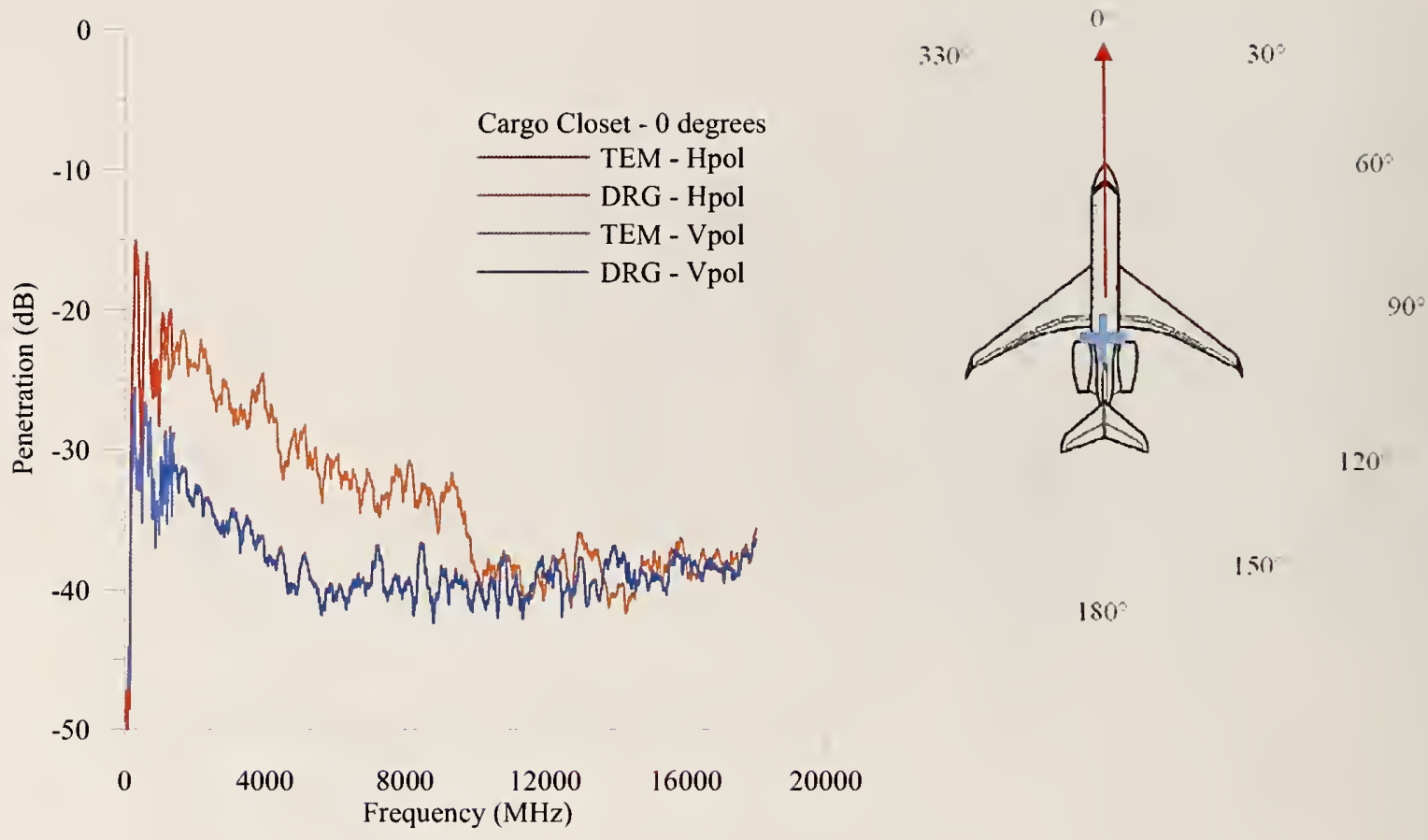

Figure 91. Penetration versus frequency for all antennas and polarizations for the transmitting tower placed at the $0^{\circ}$ position and the receiving antennas placed in the cargo closet.
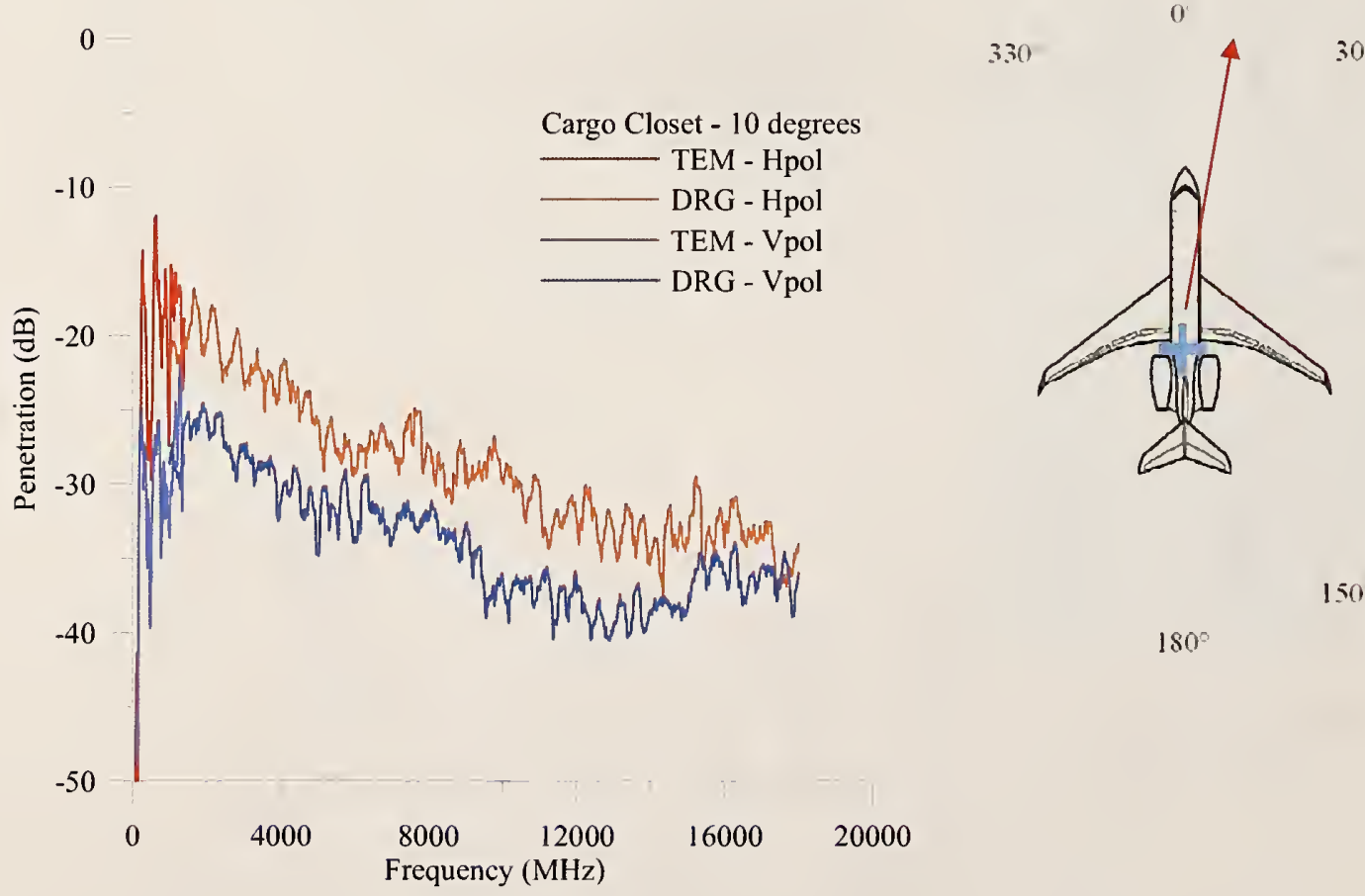

$60^{\circ}$

Figure 92. Penetration versus frequency for all antennas and polarizations for the transmitting tower placed at the $10^{\circ}$ position and the receiving antennas placed in the cargo closet. 


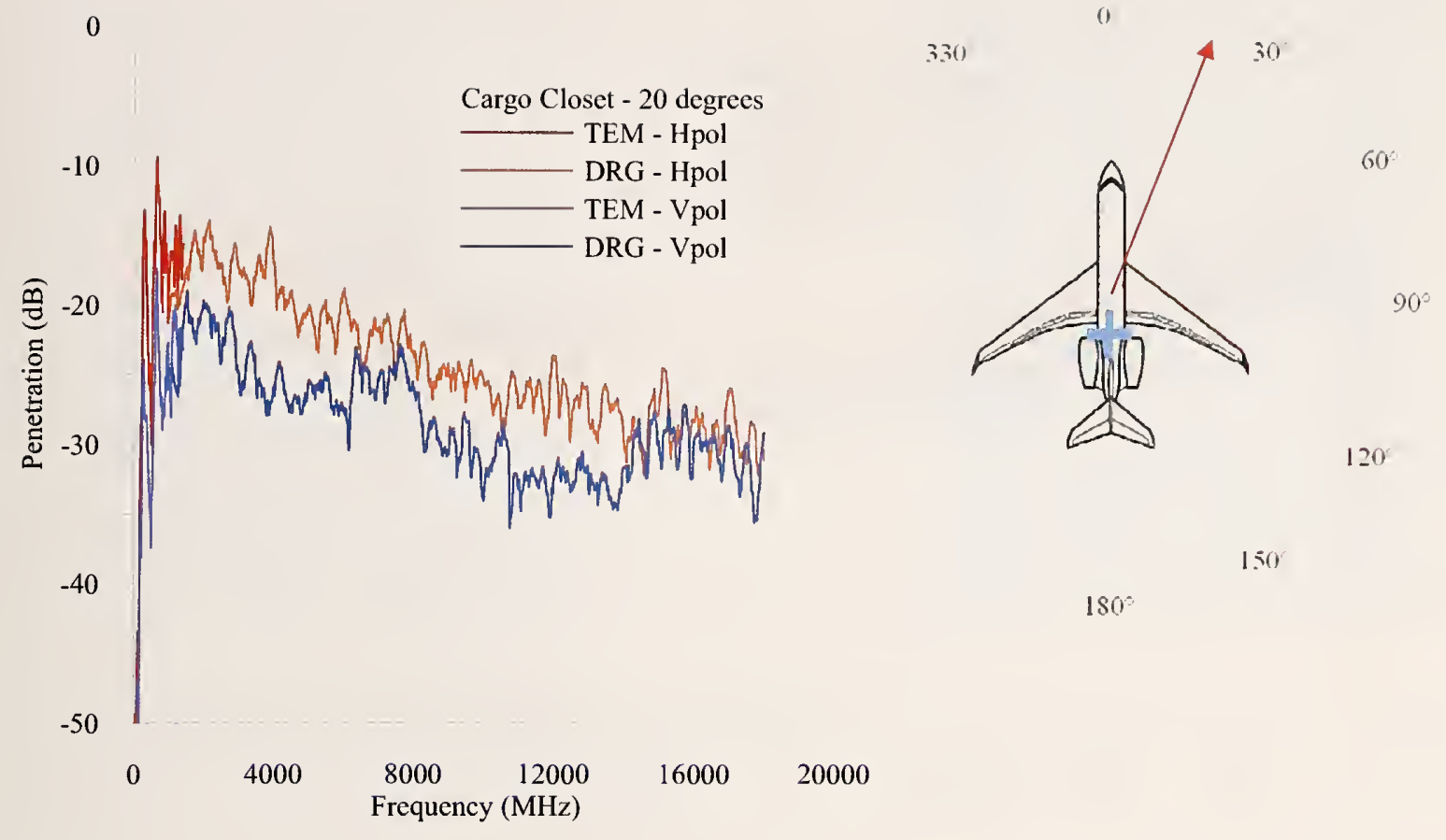

Figure 93. Penetration versus frequency for all antennas and polarizations for the transmitting tower placed at the $20^{\circ}$ position and the receiving antennas placed in the cargo closet.

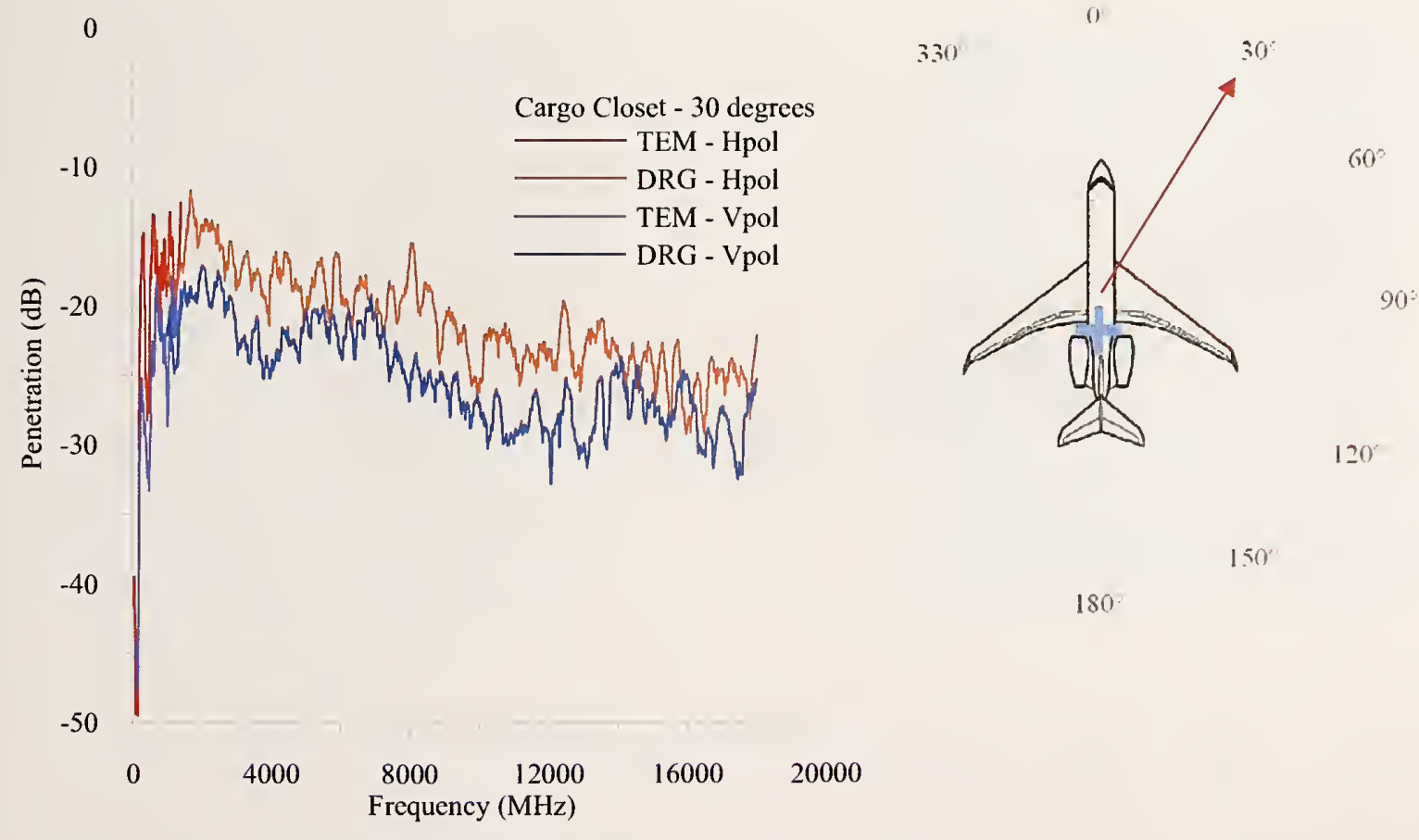

Figure 94. Penetration versus frequency for all antennas and polarizations for the transmitting tower placed at the $30^{\circ}$ position and the receiving antennas placed in the cargo closet. 
0

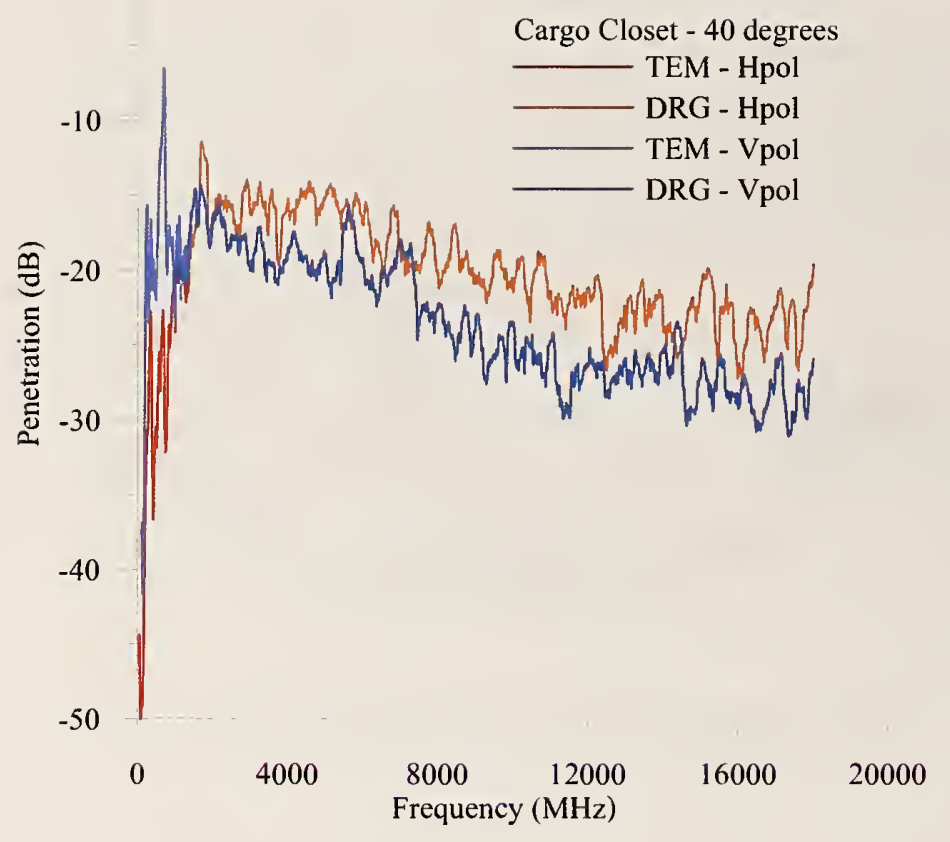

330 30

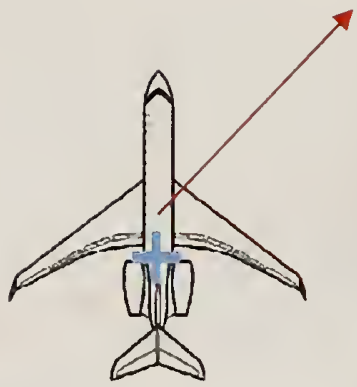

$60^{\circ}$

$90^{3}$

$120^{\circ}$

$180^{\circ}$

Figure 95. Penetration versus frequency for all antennas and polarizations for the transmitting tower placed at the $40^{\circ}$ position and the receiving antennas placed in the cargo closet.

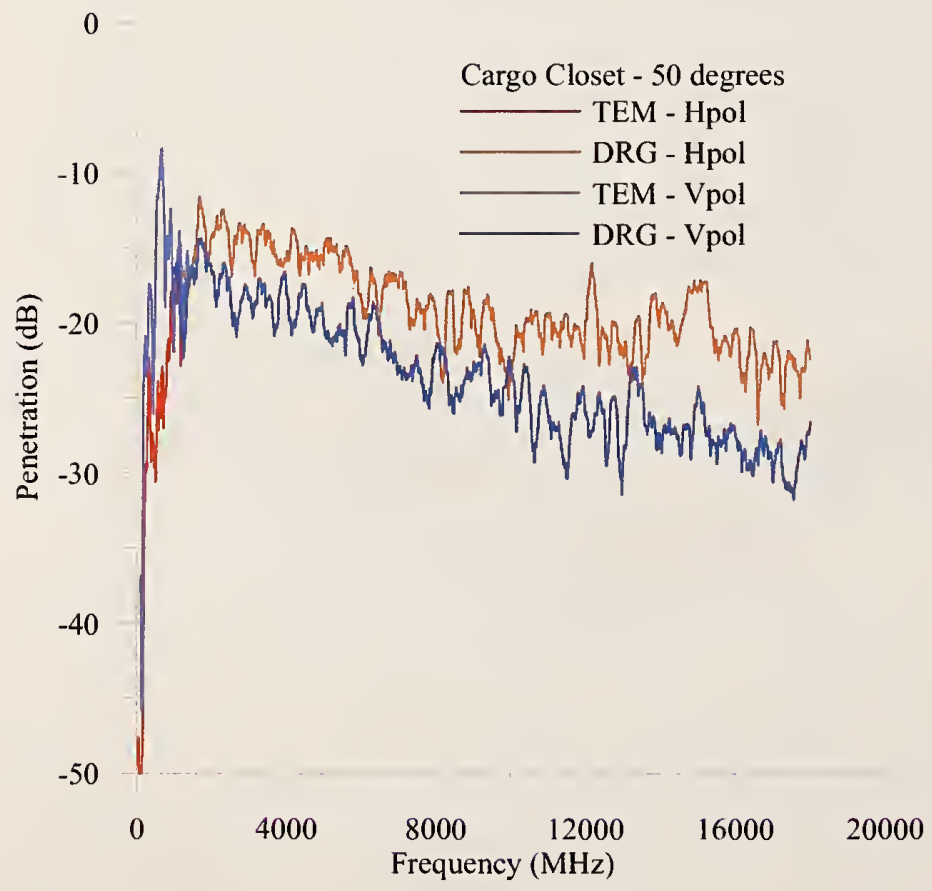

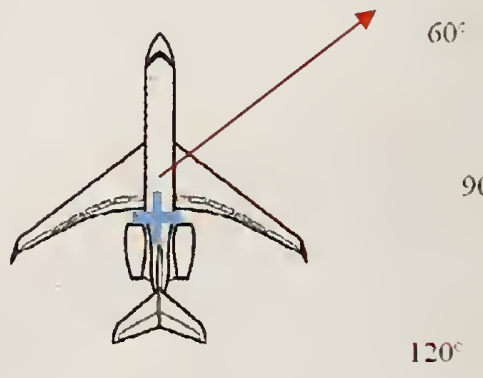

150

Figure 96. Penetration versus frequency for all antennas and polarizations for the transmitting tower placed at the $50^{\circ}$ position and the receiving antennas placed in the cargo closet. 
0

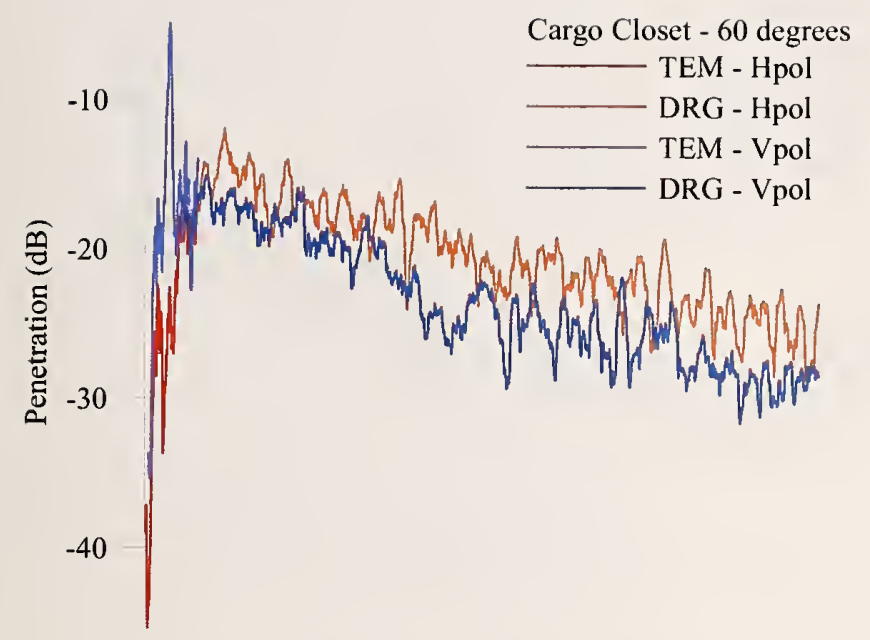

3.30

0

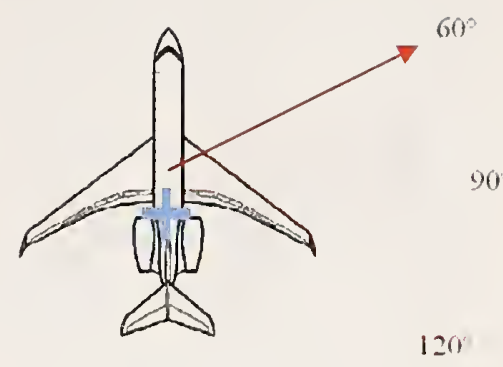

150

$-50$

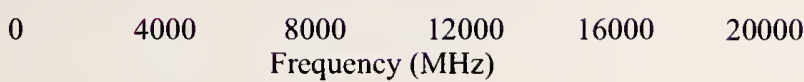

Figure 97. Penetration versus frequency for all antennas and polarizations for the transmitting tower placed at the $60^{\circ}$ position and the receiving antennas placed in the cargo closet.

0

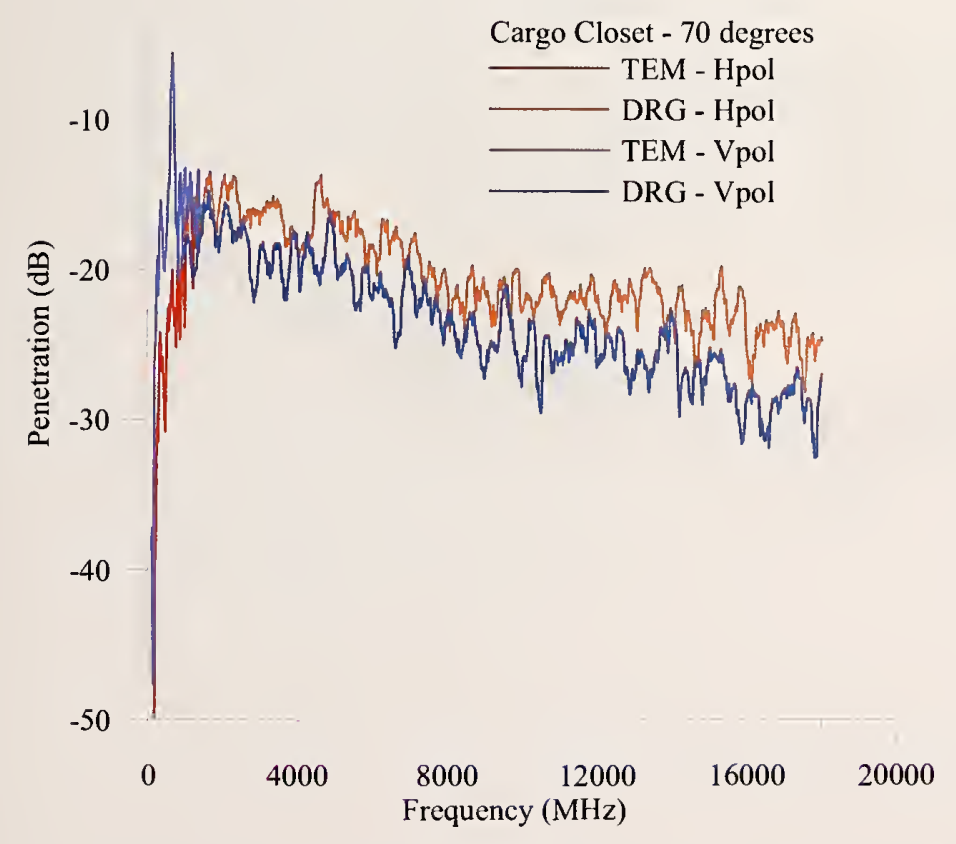

330

0
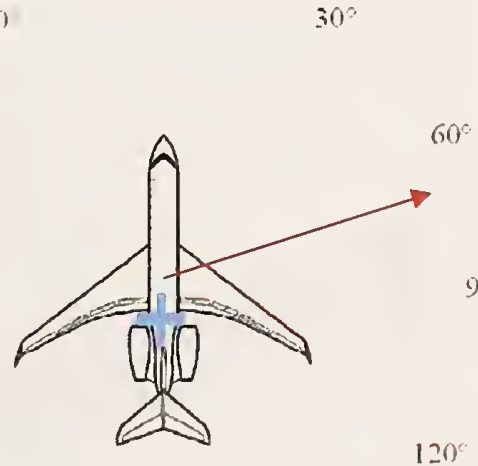

150

Figure 98. Penetration versus frequency for all antennas and polarizations for the transmitting tower placed at the $70^{\circ}$ position and the receiving antennas in the cargo closet. 
0

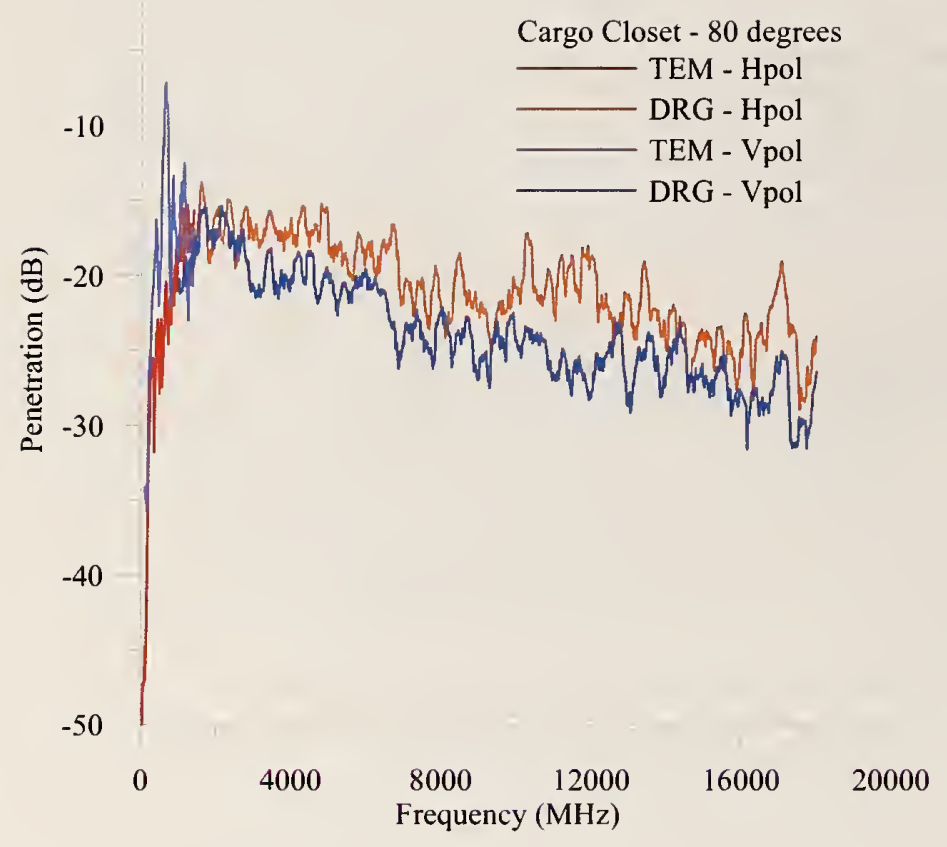

330
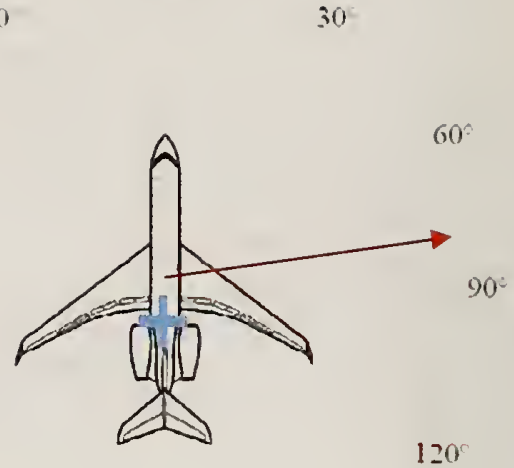

$150^{\prime}$

$180^{2}$

Figure 99. Penetration versus frequency for all antennas and polarizations for the transmitting tower placed at the $80^{\circ}$ position and the receiving antennas placed in the cargo closet.

0

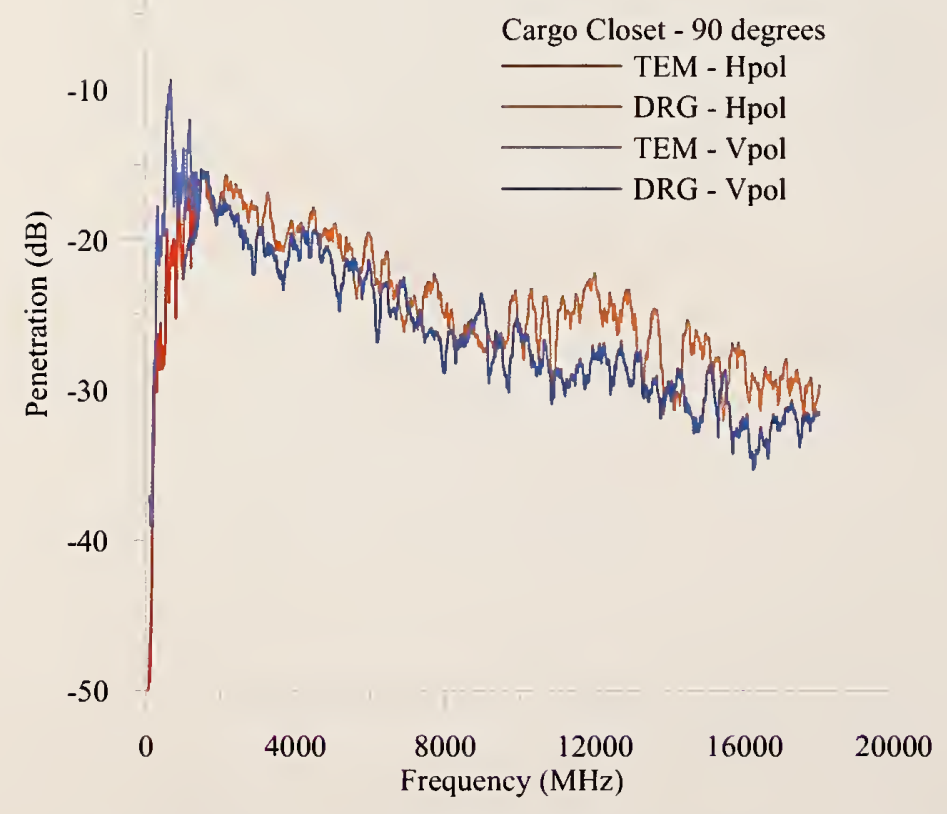

$330 \quad 30^{\circ}$

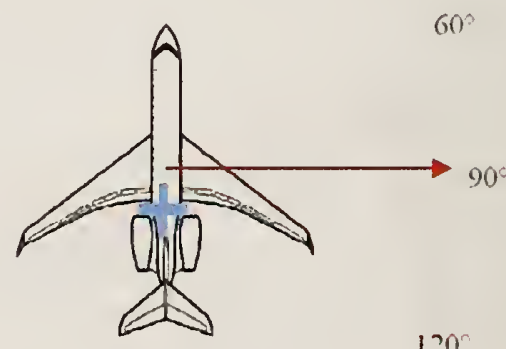

150

Figure 100. Penetration versus frequency for all antennas and polarizations for the transmitting tower placed at the $90^{\circ}$ position and the receiving antennas placed in the cargo closet. 
0

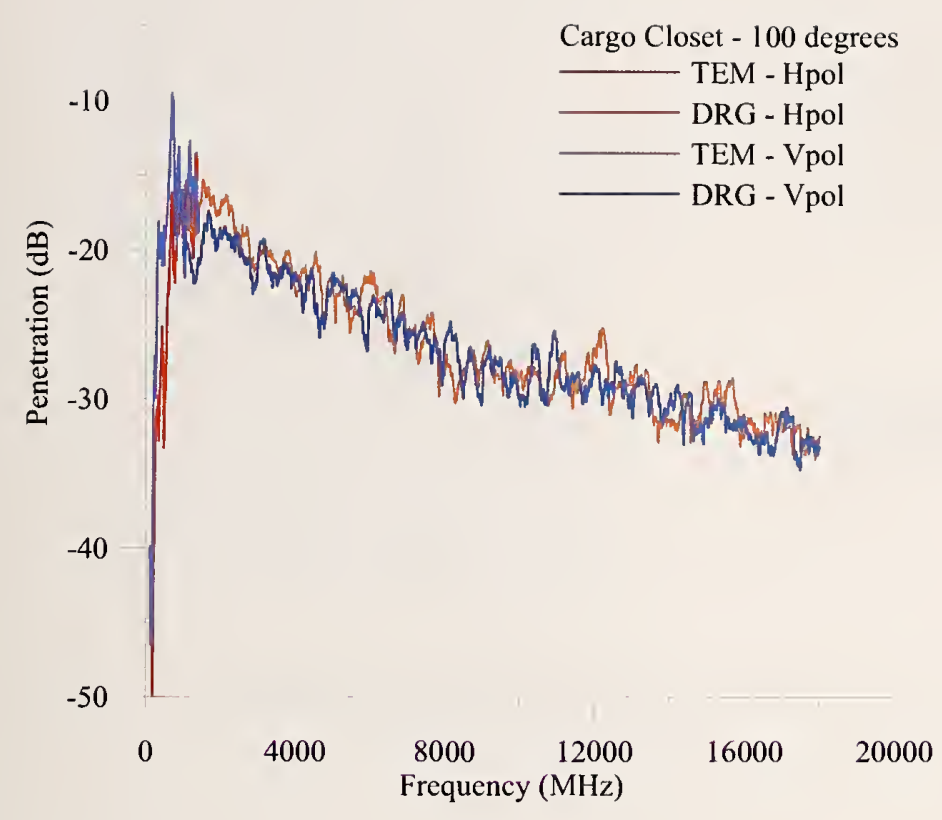

330

(1)

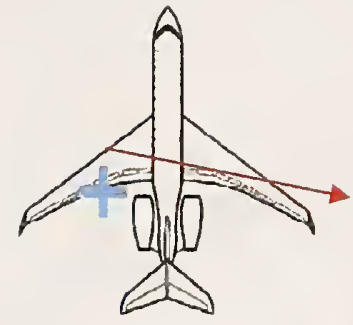

$60^{\circ}$

96

120

180

Figure 101. Penetration versus frequency for all antennas and polarizations for the transmitting tower placed at the $100^{\circ}$ position and the receiving antennas placed in the cargo closet.

0

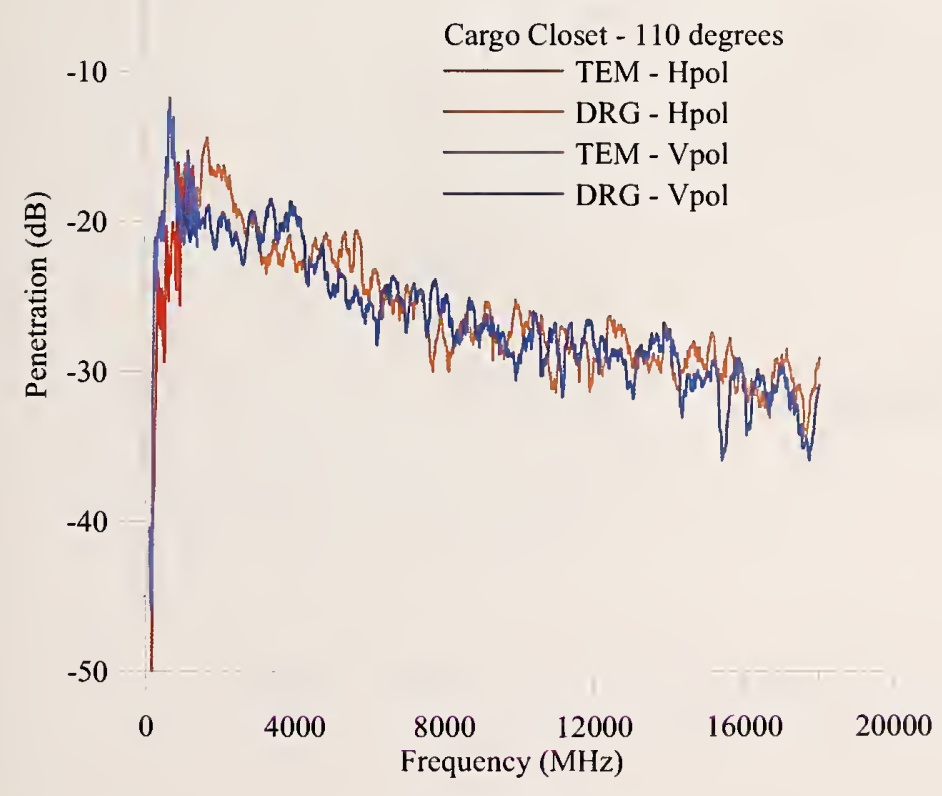

$330^{\circ} \quad 30^{\circ}$

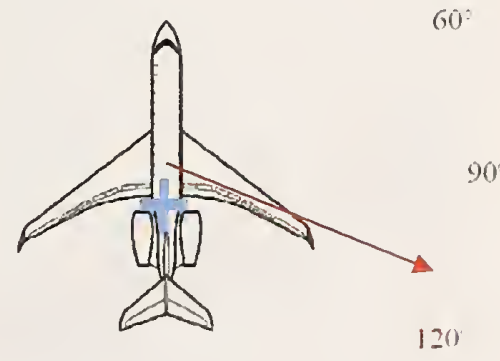

150

$180^{\circ}$

Figure 102. Penetration versus frequency for all antennas and polarizations for the transmitting tower placed at the $110^{\circ}$ position and the receiving antennas placed in the cargo closet. 
0

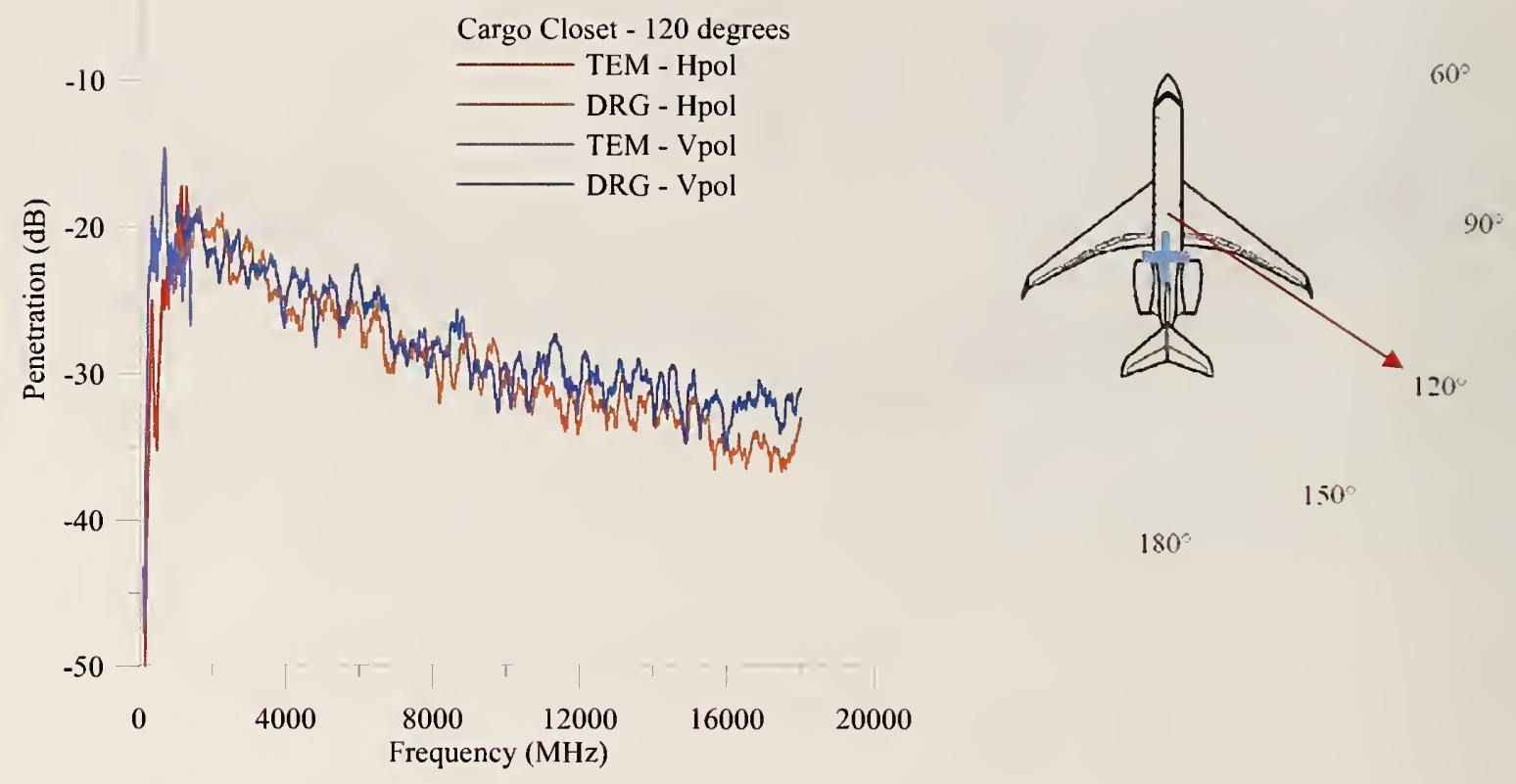

Figure 103. Penetration versus frequency for all antennas and polarizations for the transmitting tower placed at the $120^{\circ}$ position and the receiving antennas placed in the cargo closet.

0

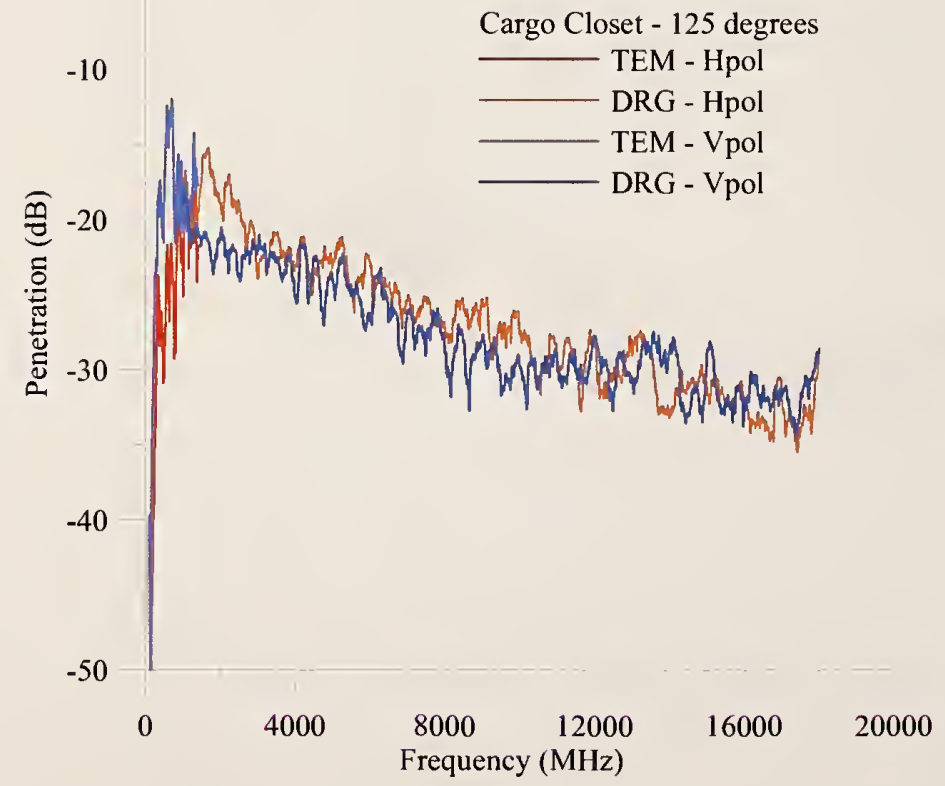

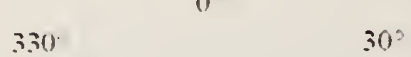

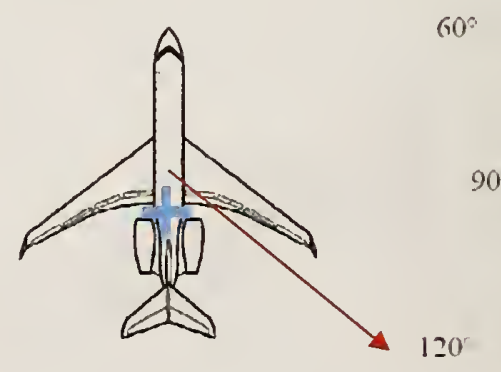

1.50

180

Figure 104. Penetration versus frequency for all antennas and polarizations for the transmitting tower placed at the $125^{\circ}$ position and the receiving antennas placed in the cargo closet. 
0

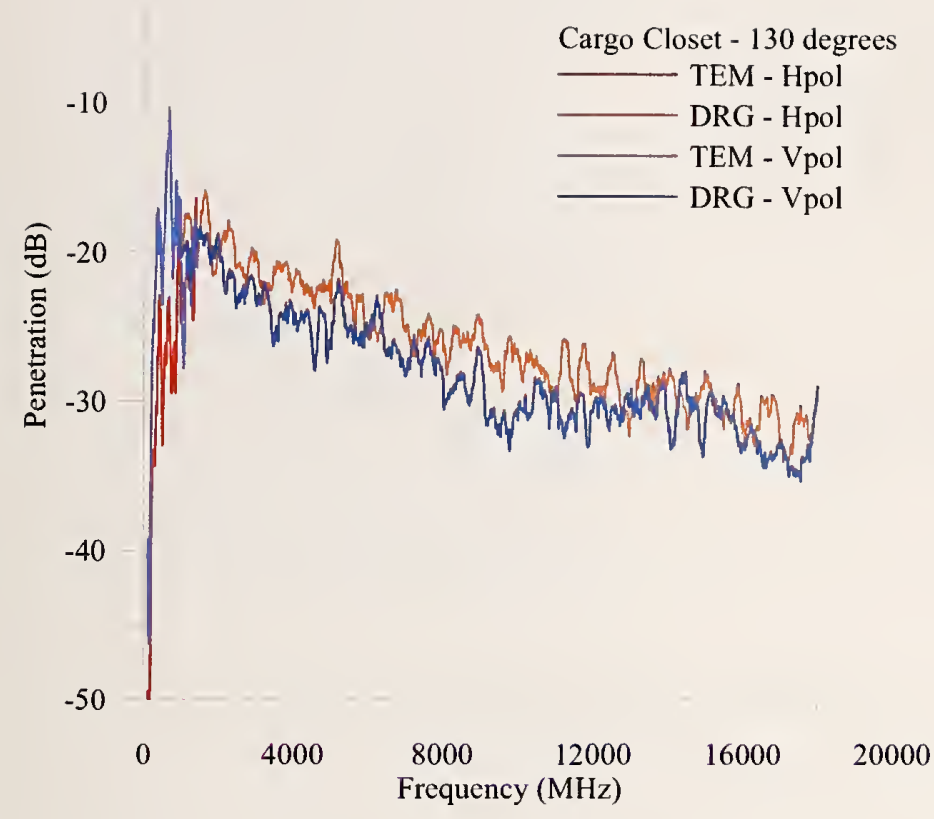

330 $30^{\circ}$

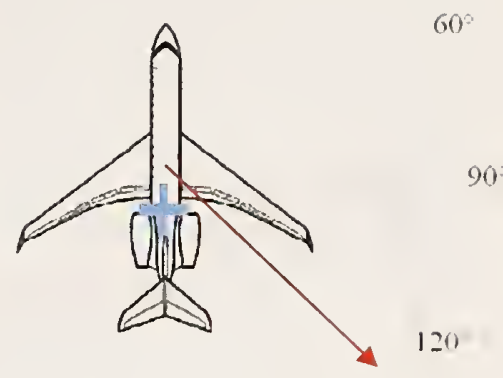

150

$180^{\circ}$

Figure 105. Penetration versus frequency for all antennas and polarizations for the transmitting tower placed at the $130^{\circ}$ position and the receiving antennas placed in the cargo closet.

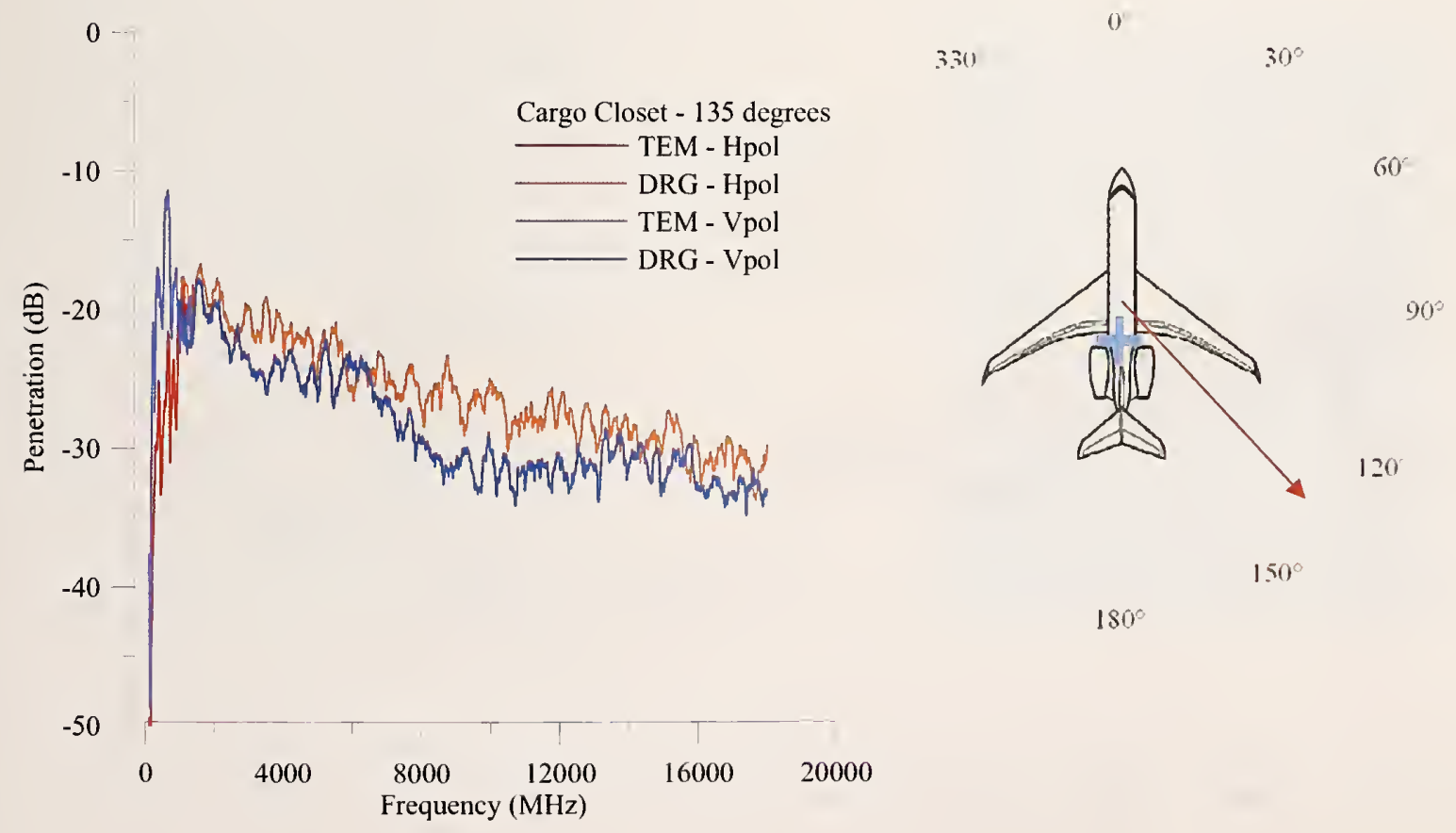

Figure 106. Penetration versus frequency for all antennas and polarizations for the transmitting tower placed at the $135^{\circ}$ position and the receiving antennas placed in the cargo closet. 

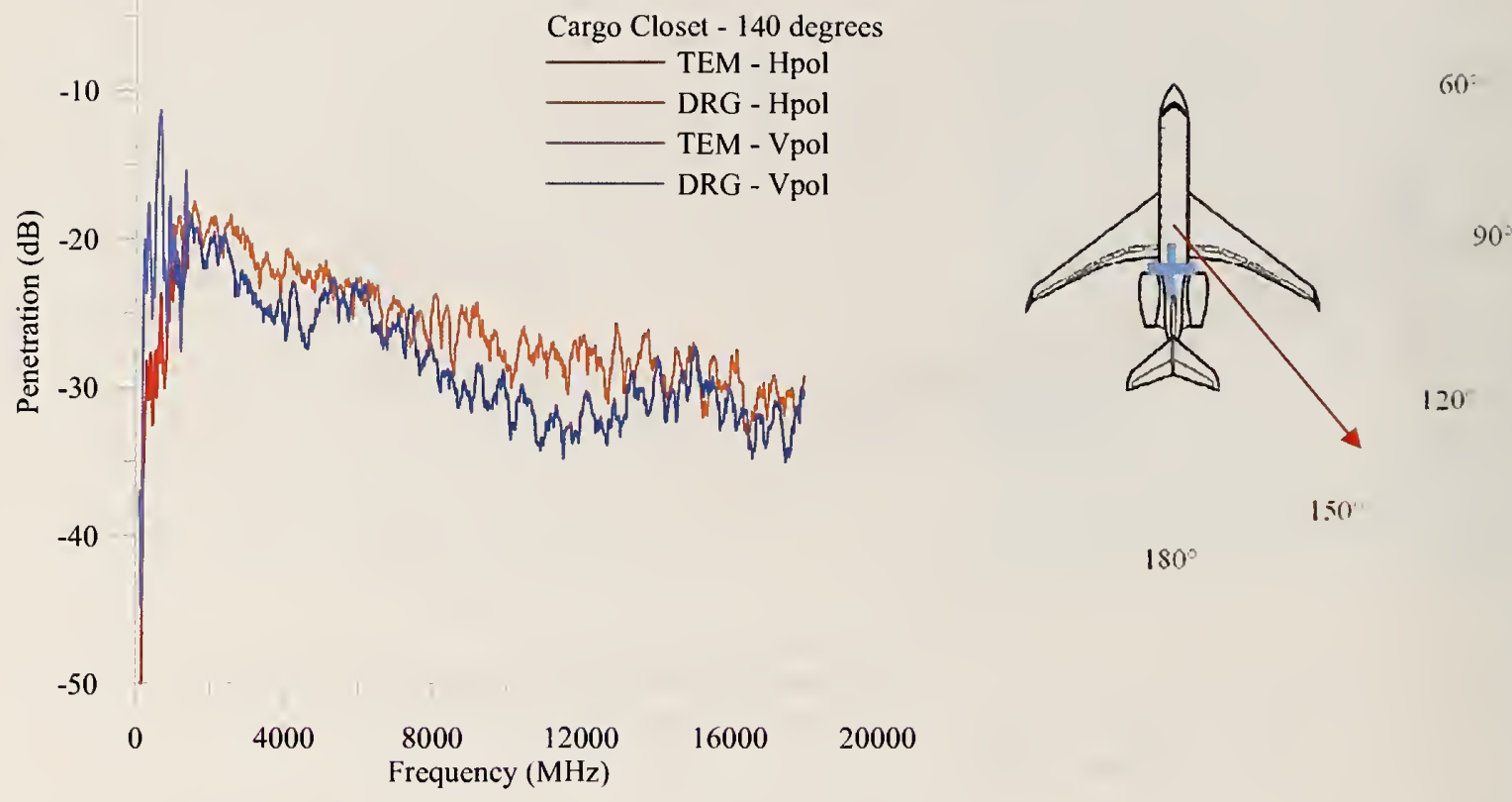

1.50

$180^{\circ}$

Figure 107. Penetration versus frequency for all antennas and polarizations for the transmitting tower placed at the $140^{\circ}$ position and the receiving antennas placed in the cargo closet.

0

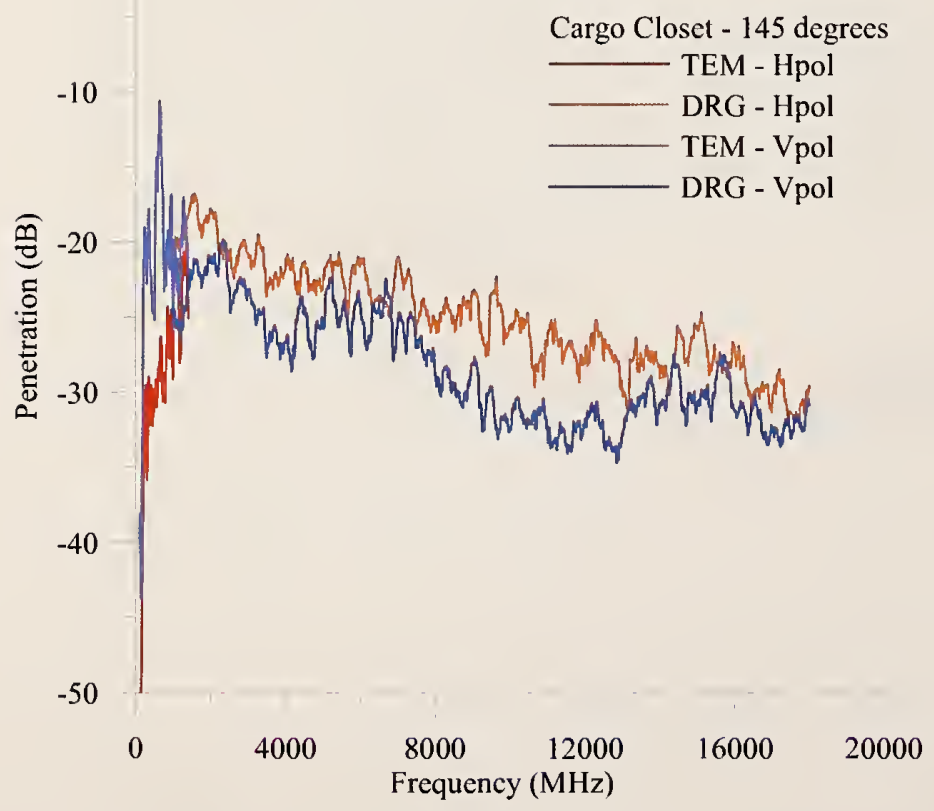

330

0

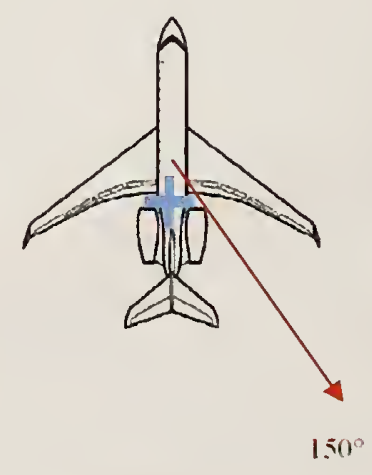

$180^{\circ}$
60

$90^{\circ}$

$120^{\circ}$

Figure 108. Penetration versus frequency for all antennas and polarizations for the transmitting tower placed at the $145^{\circ}$ position and the receiving antennas placed in the cargo closet. 

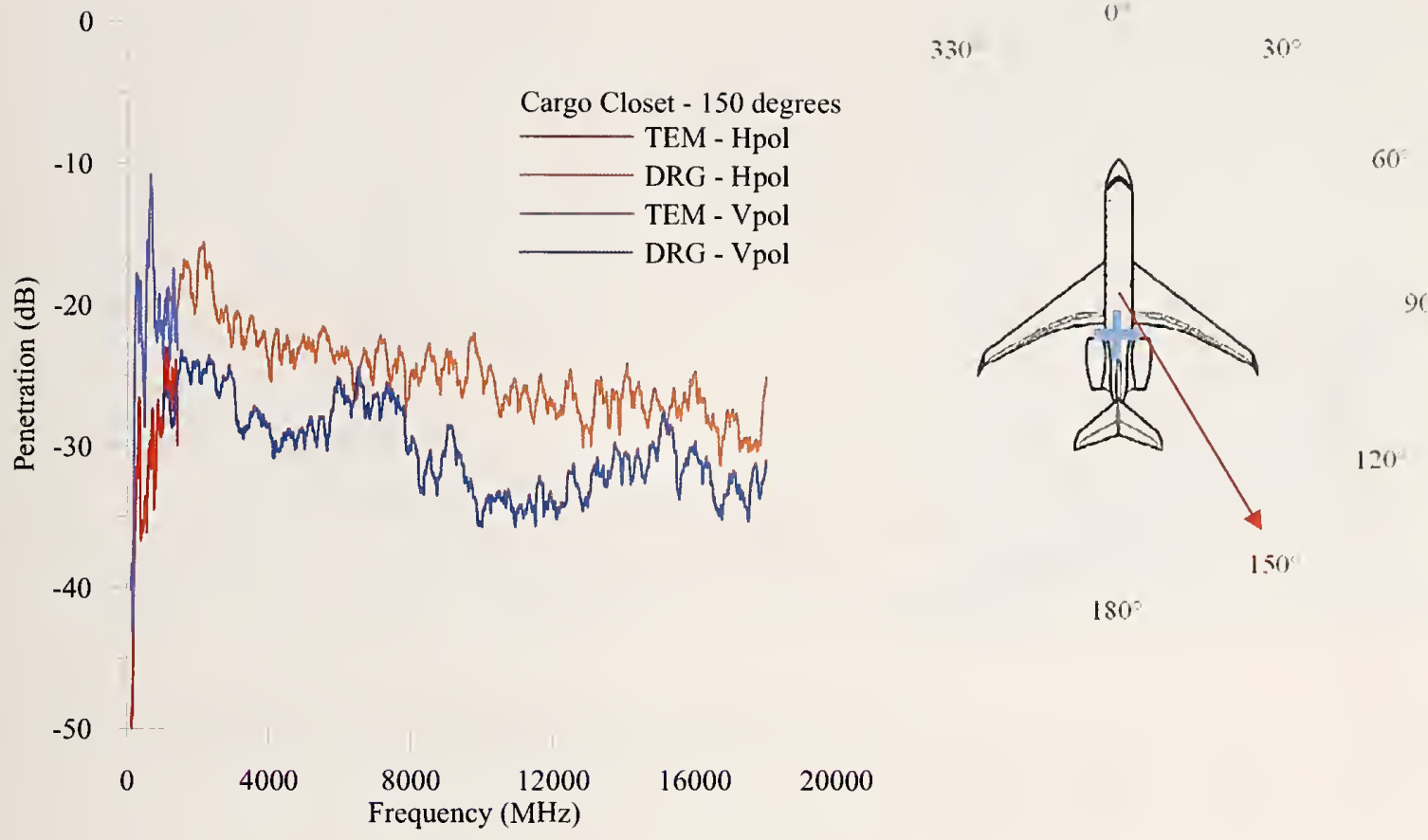

Figure 109. Penetration versus frequency for all antennas and polarizations for the transmitting tower placed at the $150^{\circ}$ position and the receiving antennas placed in the cargo closet.

0

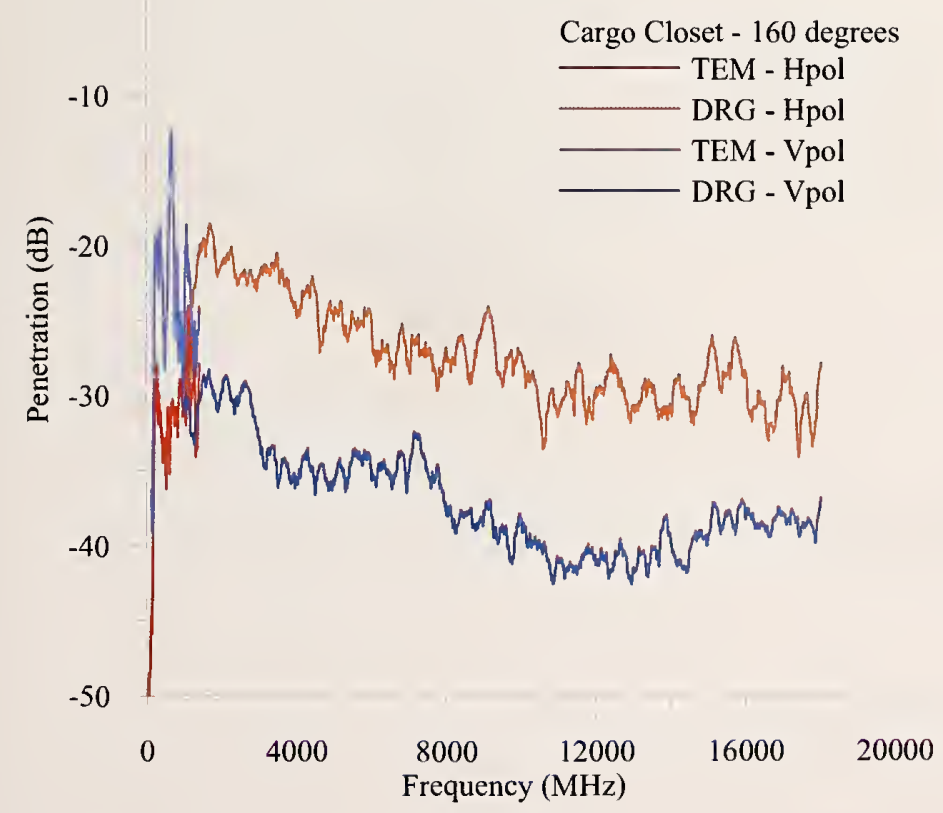

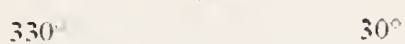

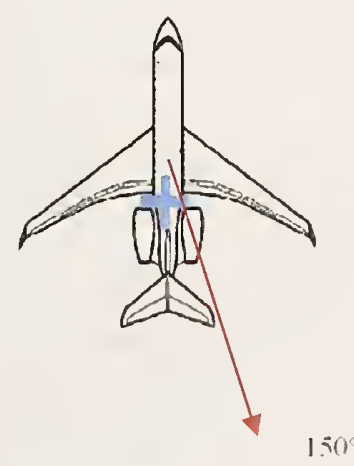

60

Figure 110. Penetration versus frequency for all antennas and polarizations for the transmitting tower placed at the $160^{\circ}$ position and the receiving antennas placed in the cargo closet. 
0

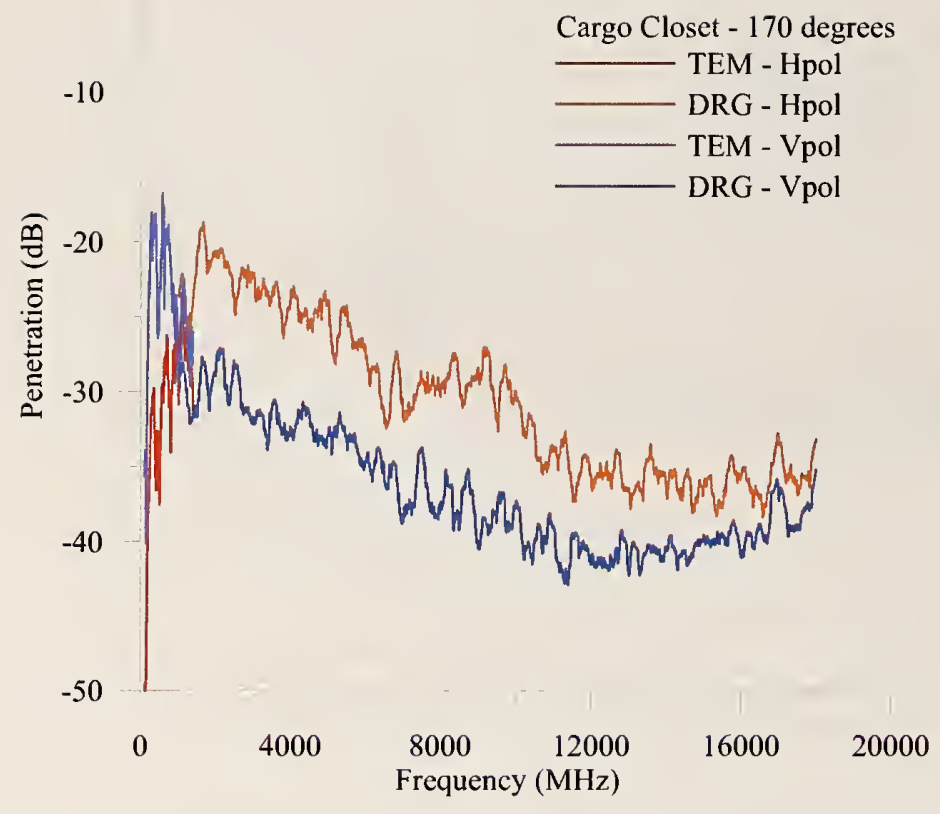

330

$30^{\circ}$

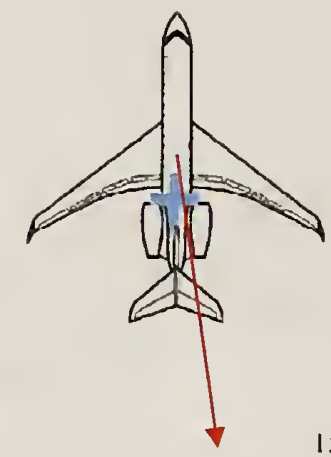

$60^{\circ}$

$90^{\circ}$

120

Figure 111. Penetration versus frequency for all antennas and polarizations for the transmitting tower placed at the $170^{\circ}$ position and the receiving antennas placed in the cargo closet.

0

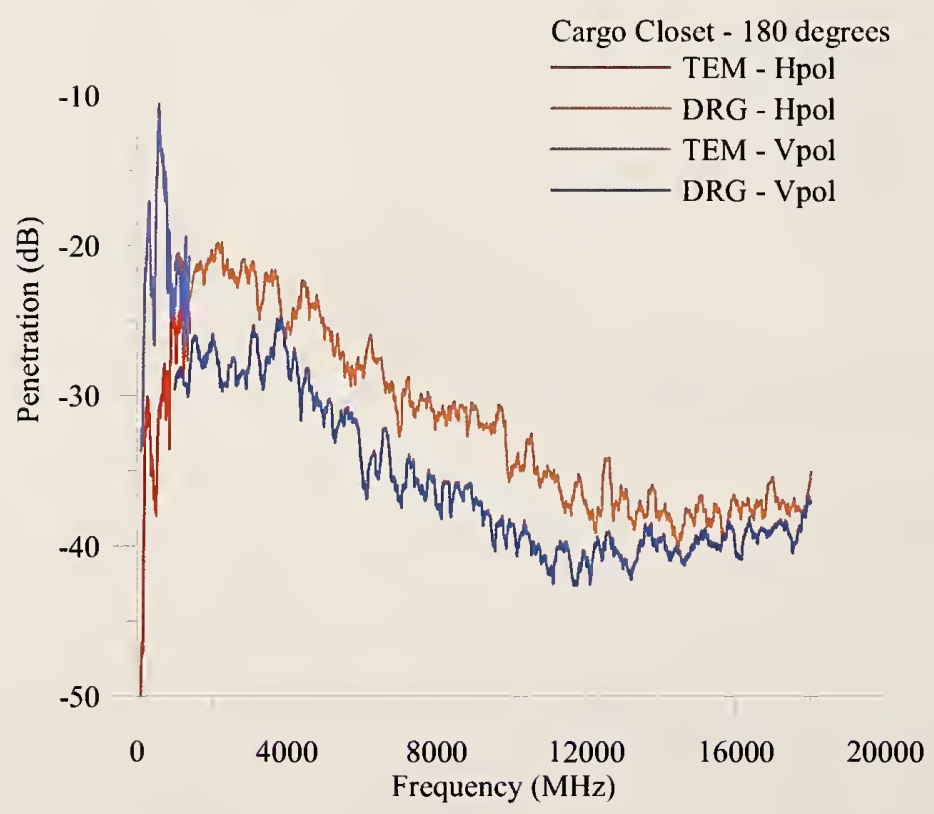

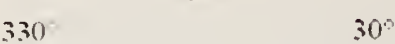

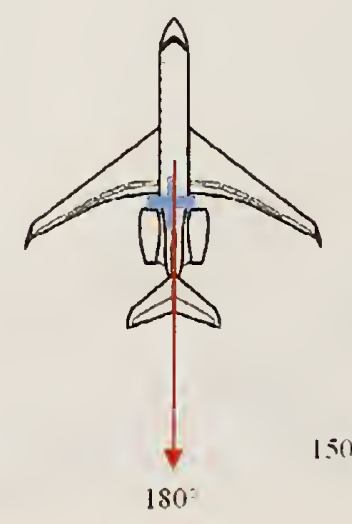

$60^{3}$

$90^{\circ}$

$120^{\circ}$

Figure 112. Penetration versus frequency for all antennas and polarizations for the transmitting tower placed at the $180^{\circ}$ position and the receiving antennas placed in the cargo closet. 
0

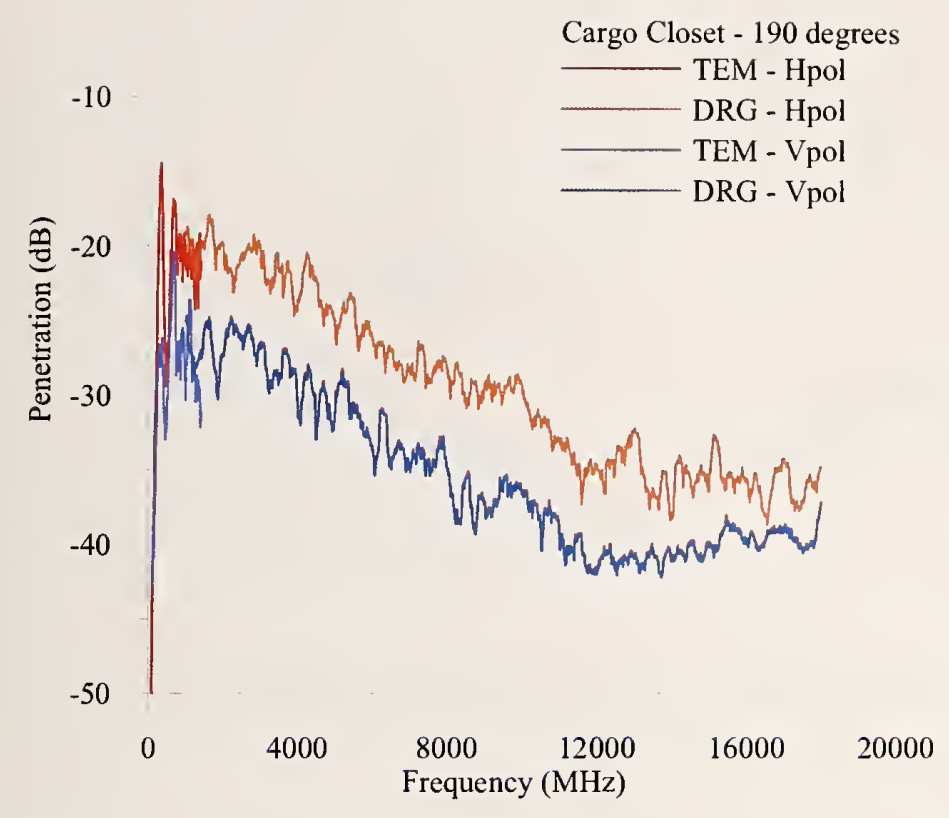

330

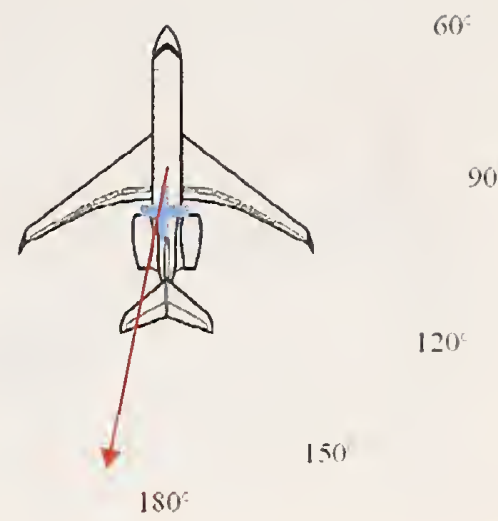

Figure 113. Penetration versus frequency for all antennas and polarizations for the transmitting tower placed at the $190^{\circ}$ position and the receiving antennas placed in the cargo closet.

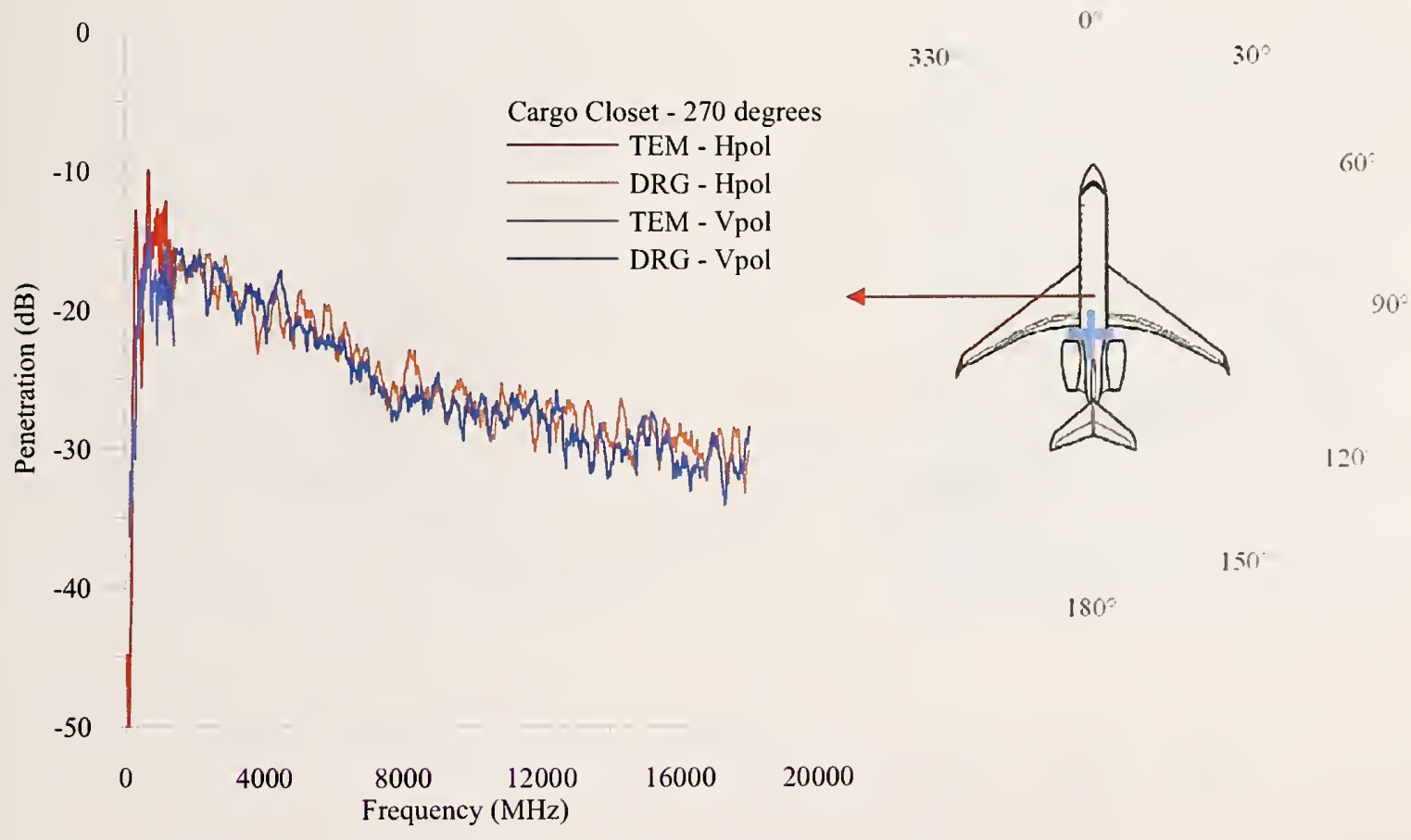

Figure 114. Penetration versus frequency for all antennas and polarizations for the transmitting tower placed at the $270^{\circ}$ position and the receiving antennas placed in the cargo closet. 
0

$$
330
$$

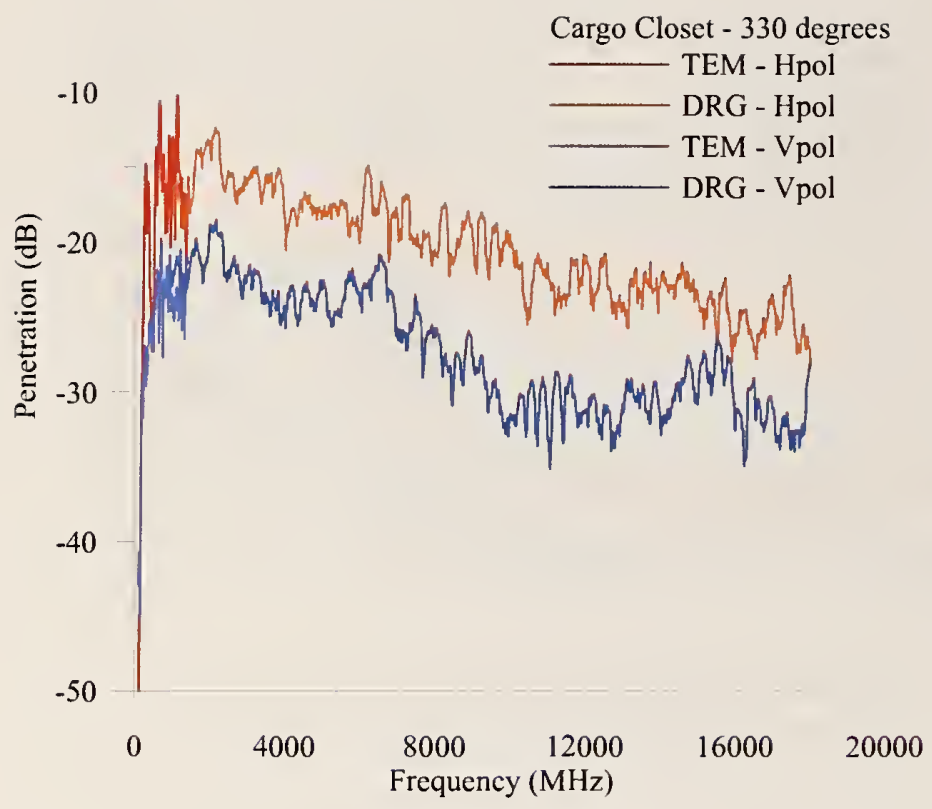

$180^{\circ}$
30

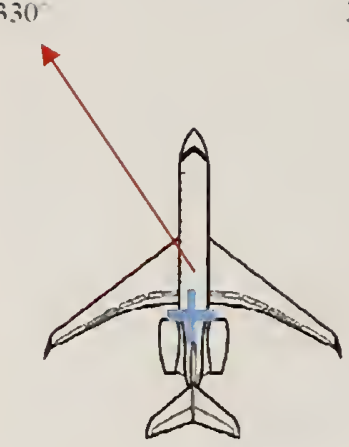

$60^{\circ}$ 90

120

1.50

Figure 115. Penetration versus frequency for all antennas and polarizations for the transmitting tower placed at the $330^{\circ}$ position and the receiving antennas placed in the cargo closet.
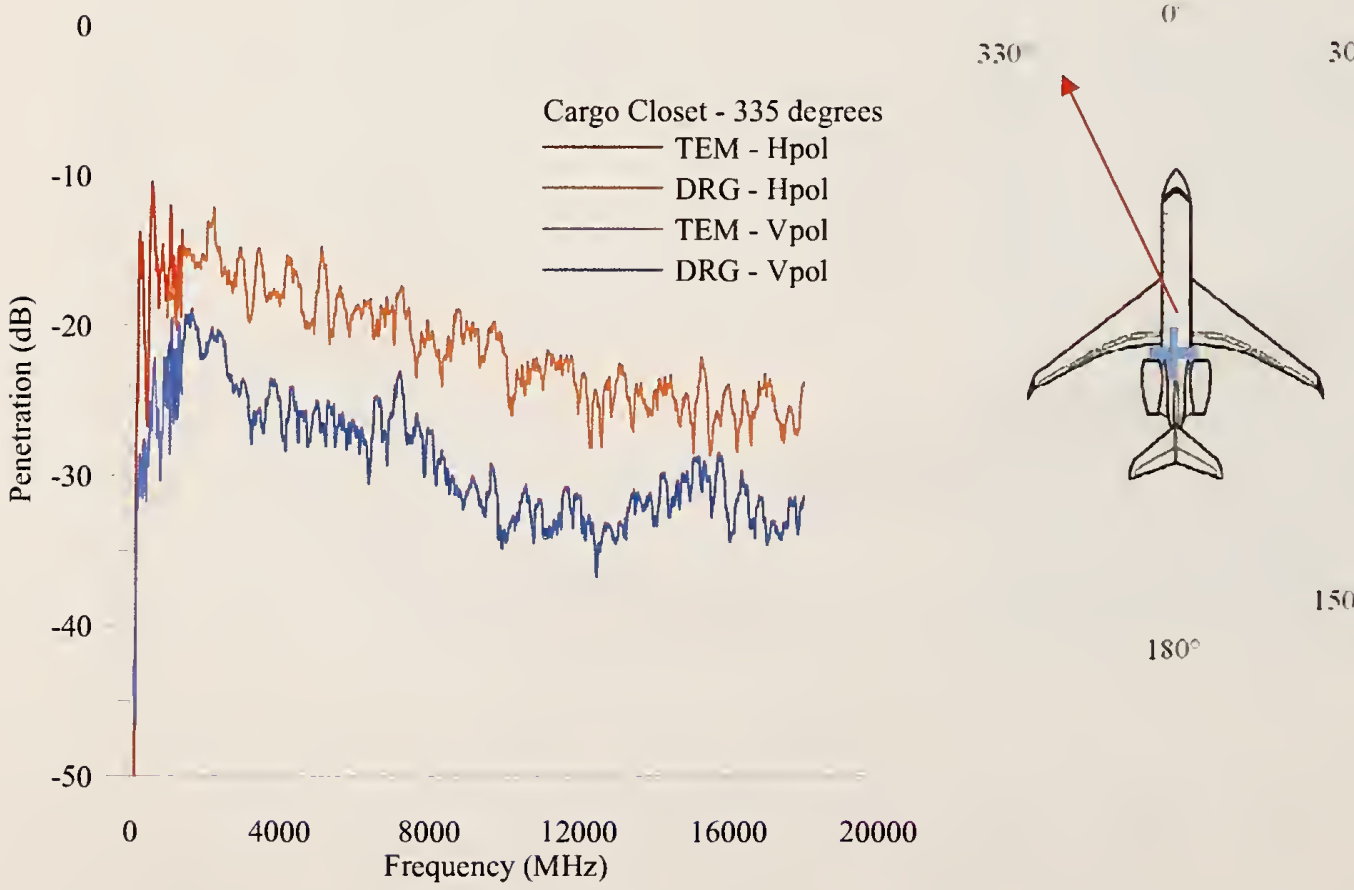

$60^{\circ}$

$90^{\circ}$

150

$180^{\circ}$

Figure 116. Penetration versus frequency for all antennas and polarizations for the transmitting tower placed at the $335^{\circ}$ position and the receiving antennas placed in the cargo closet. 


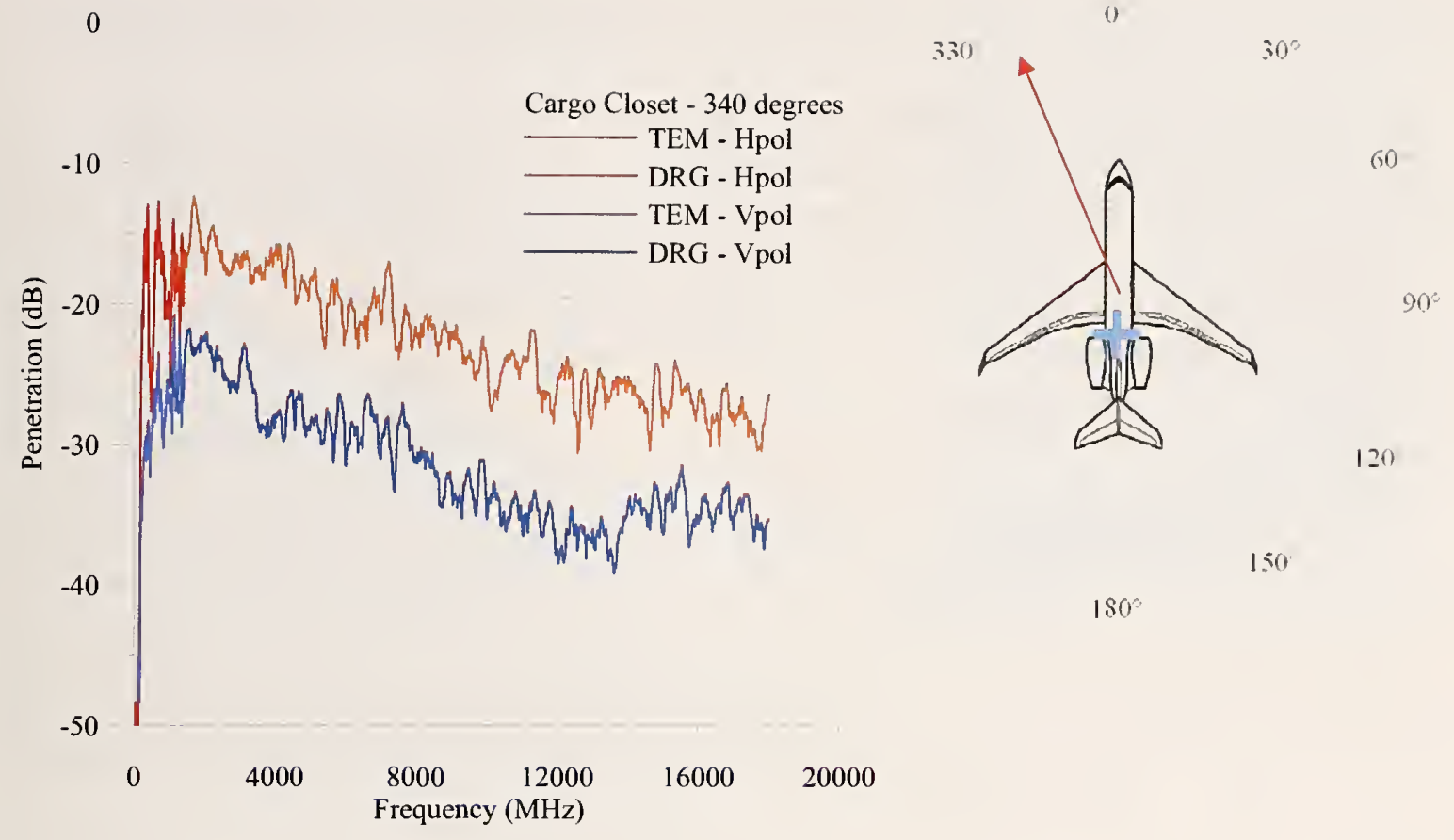

Figure 117. Penetration versus frequency for all antennas and polarizations for the transmitting tower placed at the $340^{\circ}$ position and the receiving antennas placed in the cargo closet.

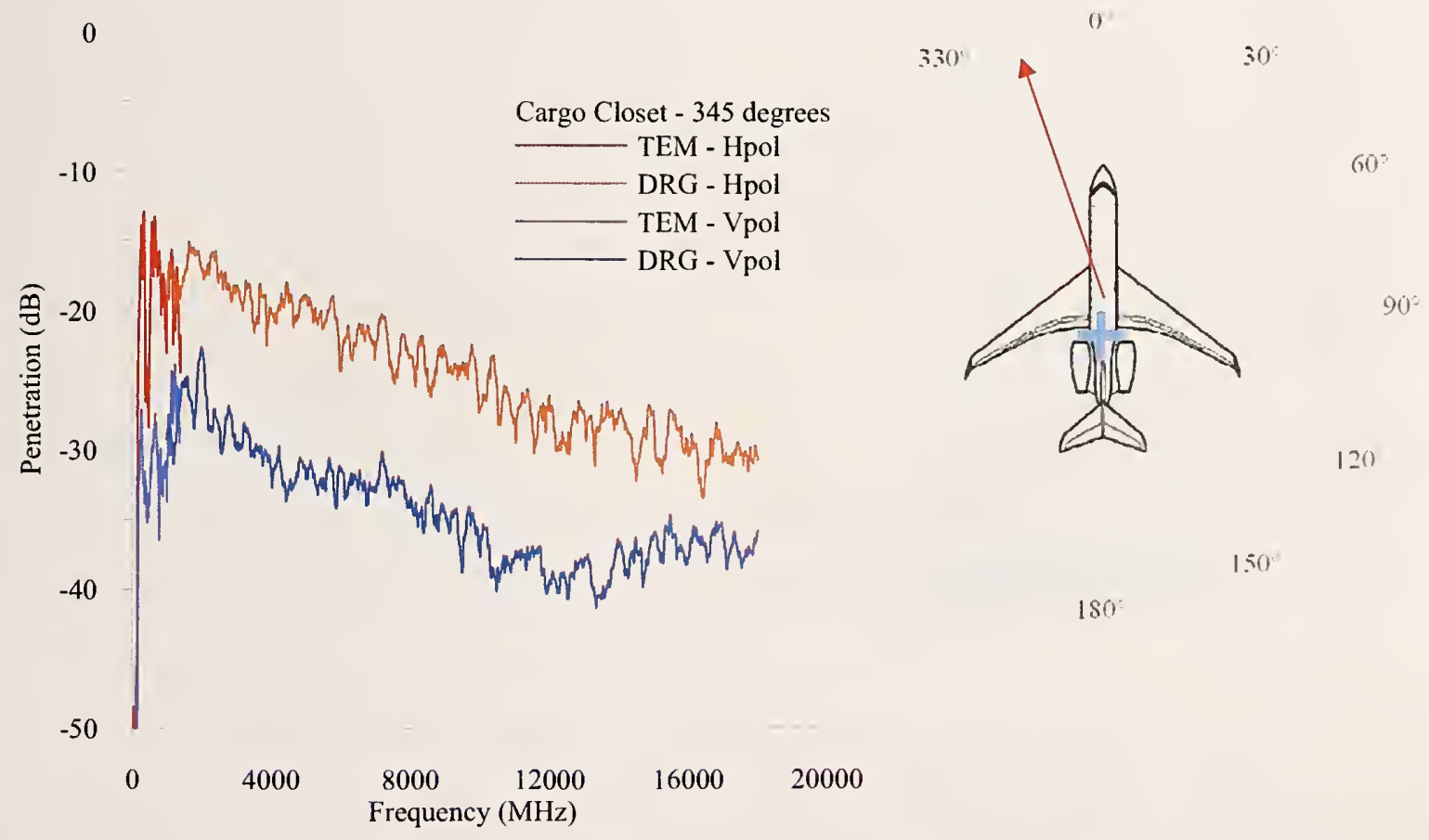

Figure 118. Penetration versus frequency for all antennas and polarizations for the transmitting tower placed at the $345^{\circ}$ position and the receiving antennas placed in the cargo closet. 


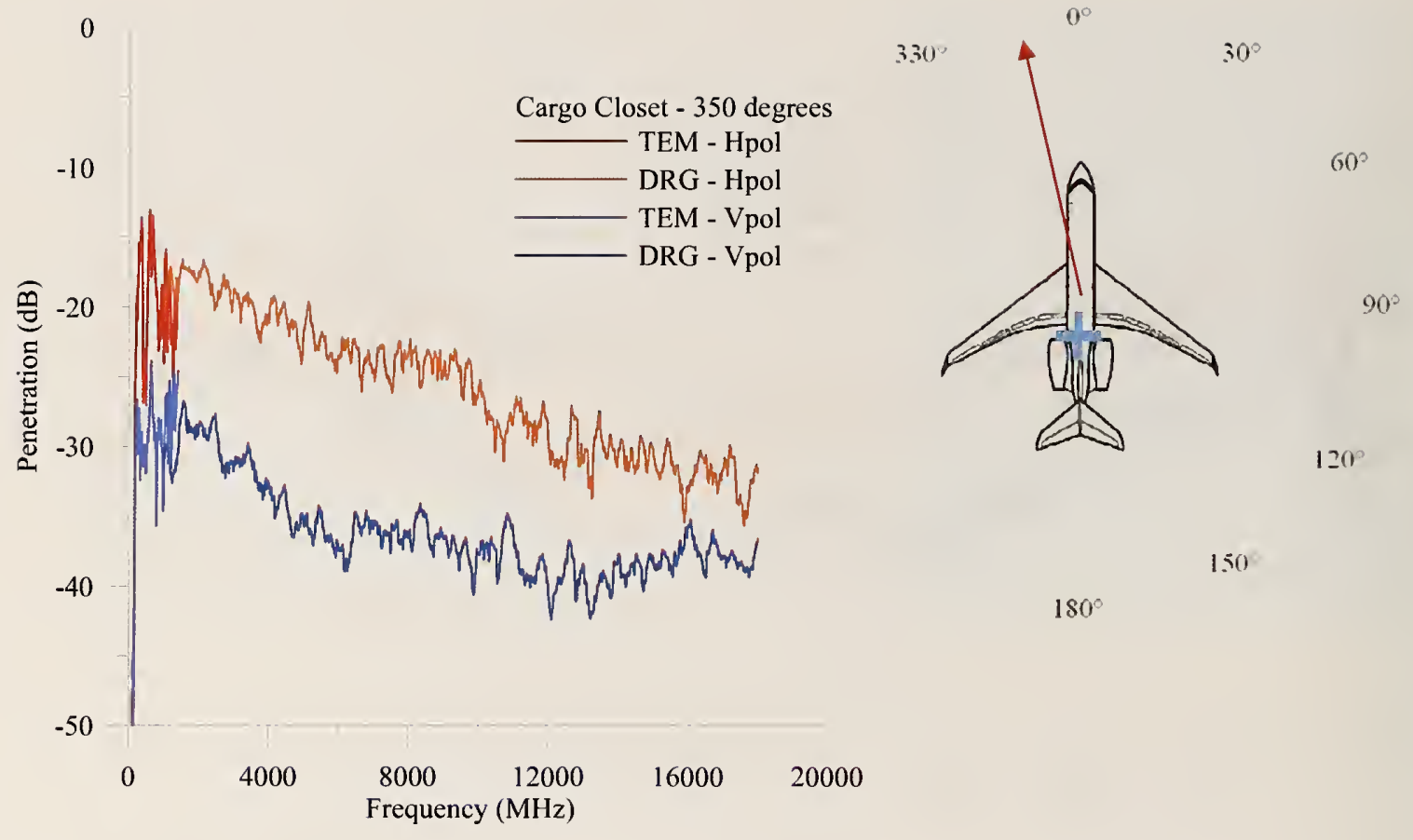

Figure 119. Penetration versus frequency for all antennas and polarizations for the transmitting tower placed at the $350^{\circ}$ position and the receiving antennas placed in the cargo closet.
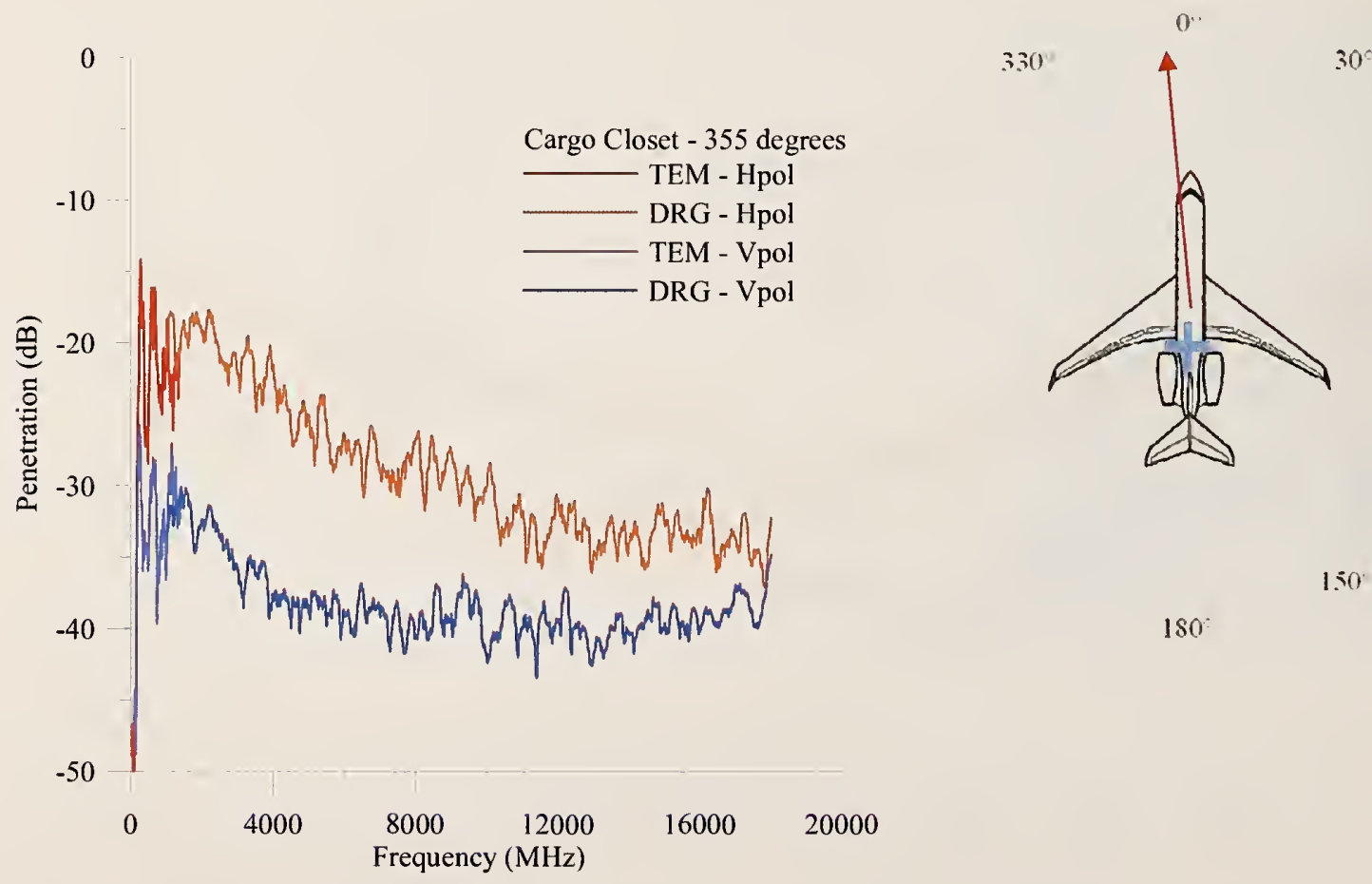

$60^{\circ}$

$90^{\circ}$

120

150

$180^{\circ}$

Figure 120. Penetration versus frequency for all antennas and polarizations for the transmitting tower placed at the $355^{\circ}$ position and the receiving antennas placed in the cargo closet. 


\subsection{HIRF Positions for Flight Deck Penetration}

These tests are standard measurements taken for HIRF evaluation of airframe shielding and vulnerability, as discussed in [1]. Five measurements were taken around the flight deck of the aircraft to investigate shielding vulnerabilities. In our measurements, we offset the transmitting antenna approximately $15 \mathrm{~m}$ from the front wheel of the aircraft. There is no required offset from the flight deck windows for testing but there are requirements for angular positions. These are at $270^{\circ}, 315^{\circ}, 0^{\circ}, 45^{\circ}$, and $90^{\circ}$ around the flight deck windows. Figure 121 shows a diagram of these measurement positions and their associated graphs. At positions $270^{\circ}$ and $315^{\circ}$ we see a sharp penetration resonance at approximately $2000 \mathrm{MHz}$ for the DRG antenna in the vertical polarization. We see that the penetration has a maximum value of approximately $-25 \mathrm{~dB}$ at around $2000 \mathrm{MHz}$ for the vertical polarization and around $3000 \mathrm{MHz}$ for the horizontal polarization. The minimum penetration values that occur at the higher frequencies are approximately $-30 \mathrm{~dB}$ to $-35 \mathrm{~dB}$. For angles $0^{\circ}, 45^{\circ}$, and $90^{\circ}$ we do not see the sharp resonance for the DRG antenna in the vertical polarization. These positions have lower penetrations of approximately $-30 \mathrm{~dB}$ at the lower frequencies and -30 to $-40 \mathrm{~dB}$ at the higher frequencies. Detailed HIRF penetration data are given in Figure 122 to 126. 


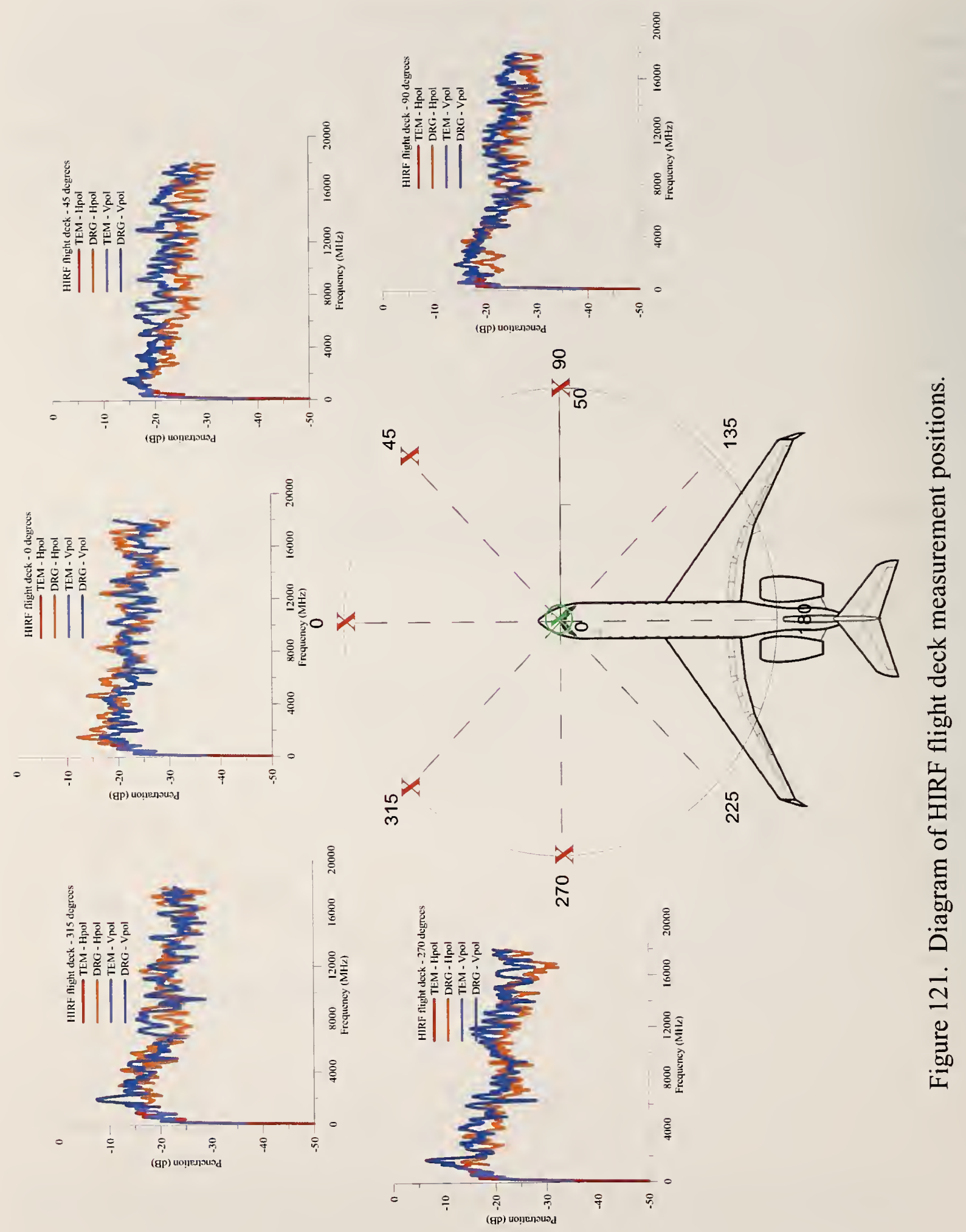




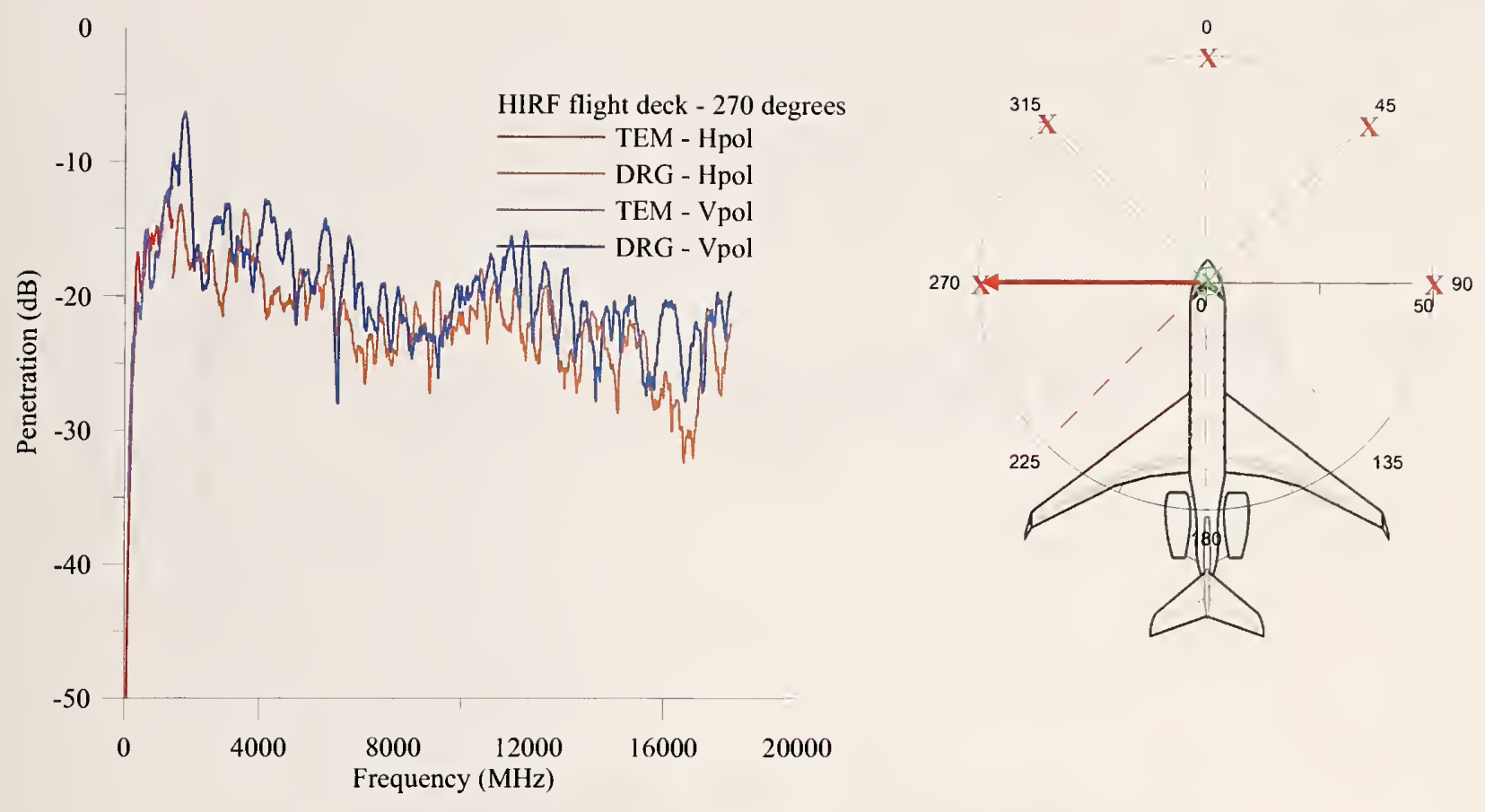

Figure 122. HIRF penetration data versus frequency for all antennas and polarizations for the transmitting tower placed at the $270^{\circ}$ position, at a distance of $15 \mathrm{~m}$ centered on the front landing gear, and the receiving antennas placed in the flight deck.

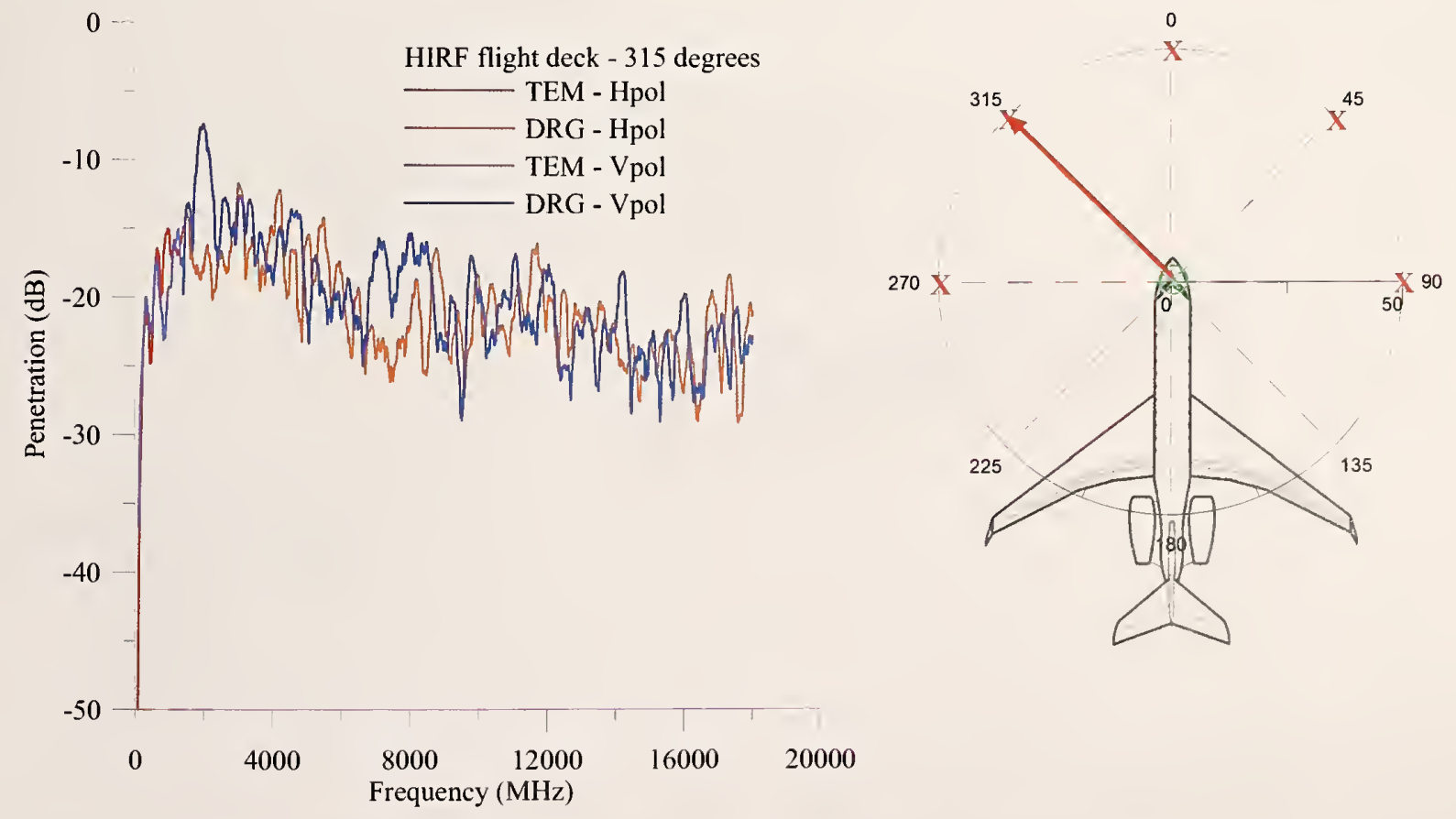

Figure 123. HIRF penetration data versus frequency for all antennas and polarizations for the transmitting tower placed at the $315^{\circ}$ position, at a distance of $15 \mathrm{~m}$ centered on the front landing gear, and the receiving antennas placed in the flight deck. 


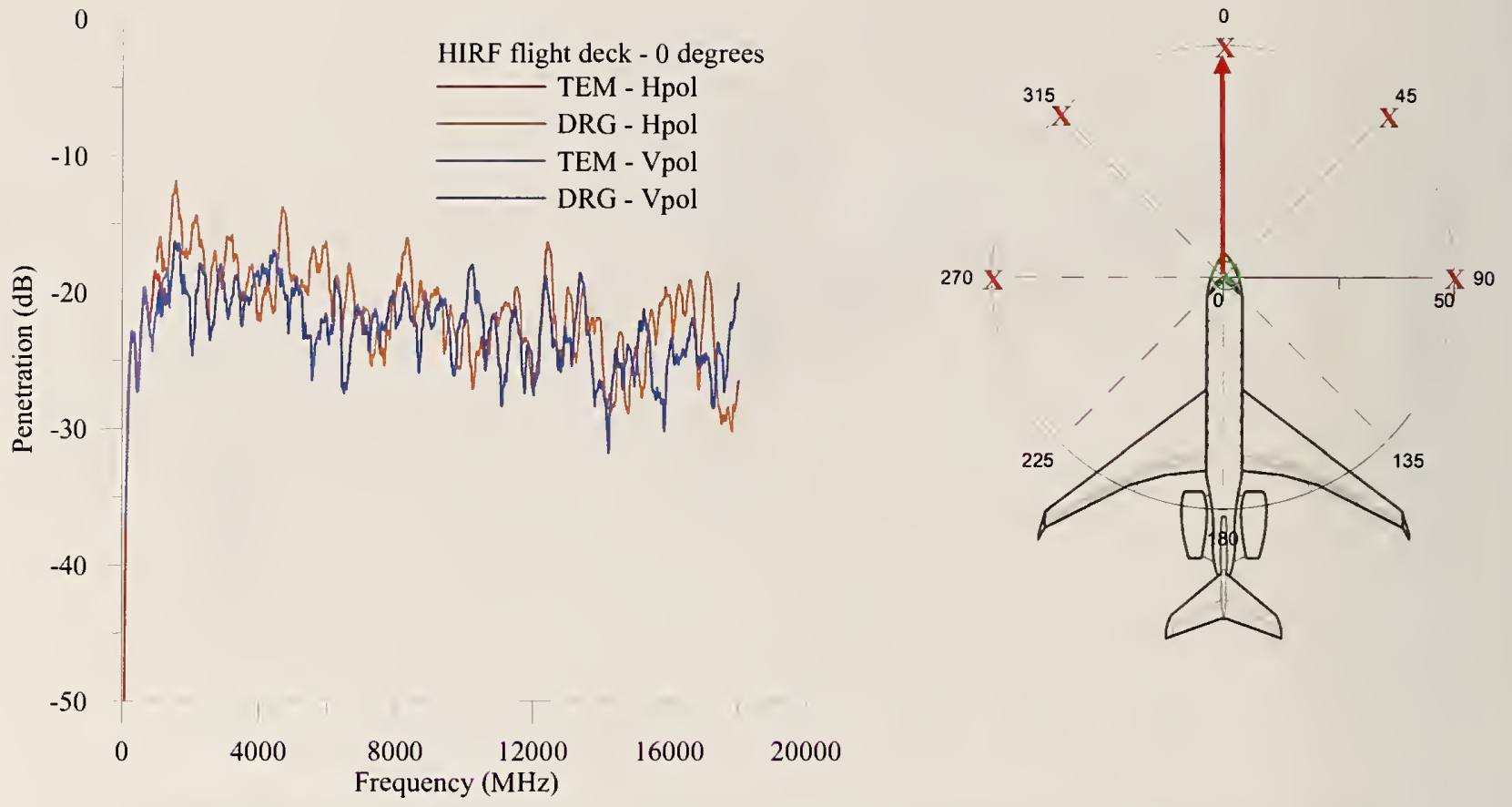

Figure 124. HIRF penetration data versus frequency for all antennas and polarizations for the transmitting tower placed at the $0^{\circ}$ position, at a distance of $15 \mathrm{~m}$ centered on the front landing gear, and the receiving antennas placed in the flight deck.

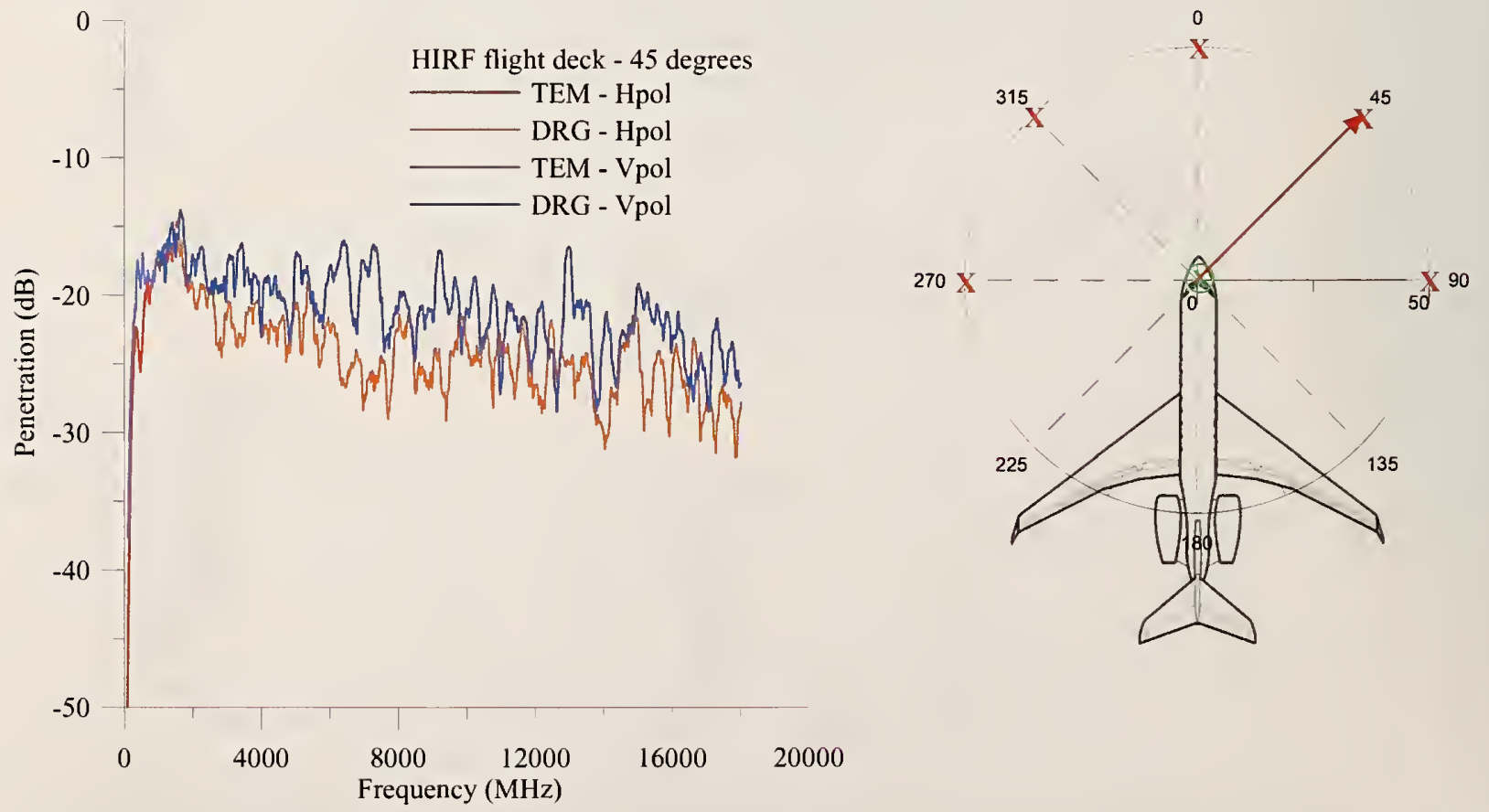

Figure 125. HIRF penetration data versus frequency for all antennas and polarizations for the transmitting tower placed at the $45^{\circ}$ position, at a distance of $15 \mathrm{~m}$ centered on the front landing gear, and the receiving antennas placed in the flight deck. 


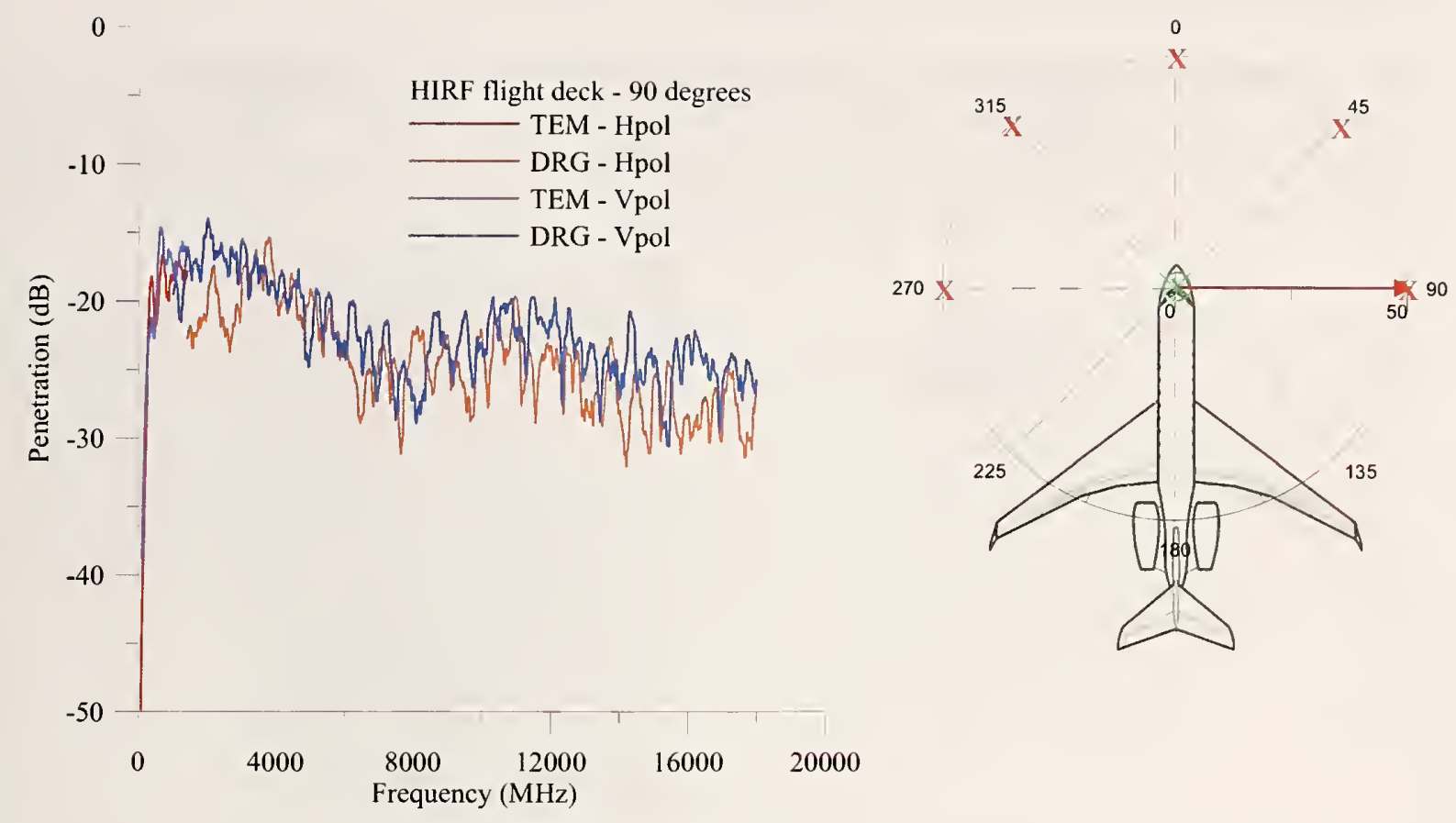

Figure 126. HIRF penetration data versus frequency for all antennas and polarizations for the transmitting tower placed at the 90 degree position, at a distance of $15 \mathrm{~m}$ centered on the front landing gear, and the receiving antennas placed in the flight deck. 


\subsection{Internal Coupling Measurements}

Internal coupling measurements can tell us a lot about the propagation environment within the airframe. It can tell us whether fields are uniform and decay at a rapid rate or more slowly over time. It can also tell us how well the fields propagate within the airframe and whether they can easily reach critical components inside the airframe.

NIST conducted several internal coupling measurements to determine the decay time and the quality factor of the internal airframe volume. We looked at the reverberant environment and how the fields couple from one cavity to another. Each configuration is shown in Figures 127 to 132. The chosen configurations were meant to physically stir the fields inside the aircraft as much as possible. In each configuration, the red rectangle represents the transmitting $1.2 \mathrm{~m}$ TEM horn antenna, the blue rectangle represents the receiving $36 \mathrm{~cm}$ TEM horn antenna, and the other two antennas are the transmitting DRG antenna (red), and the receiving DRG antenna (blue). The arrows indicate the aperture of the antenna.

Configuration 1
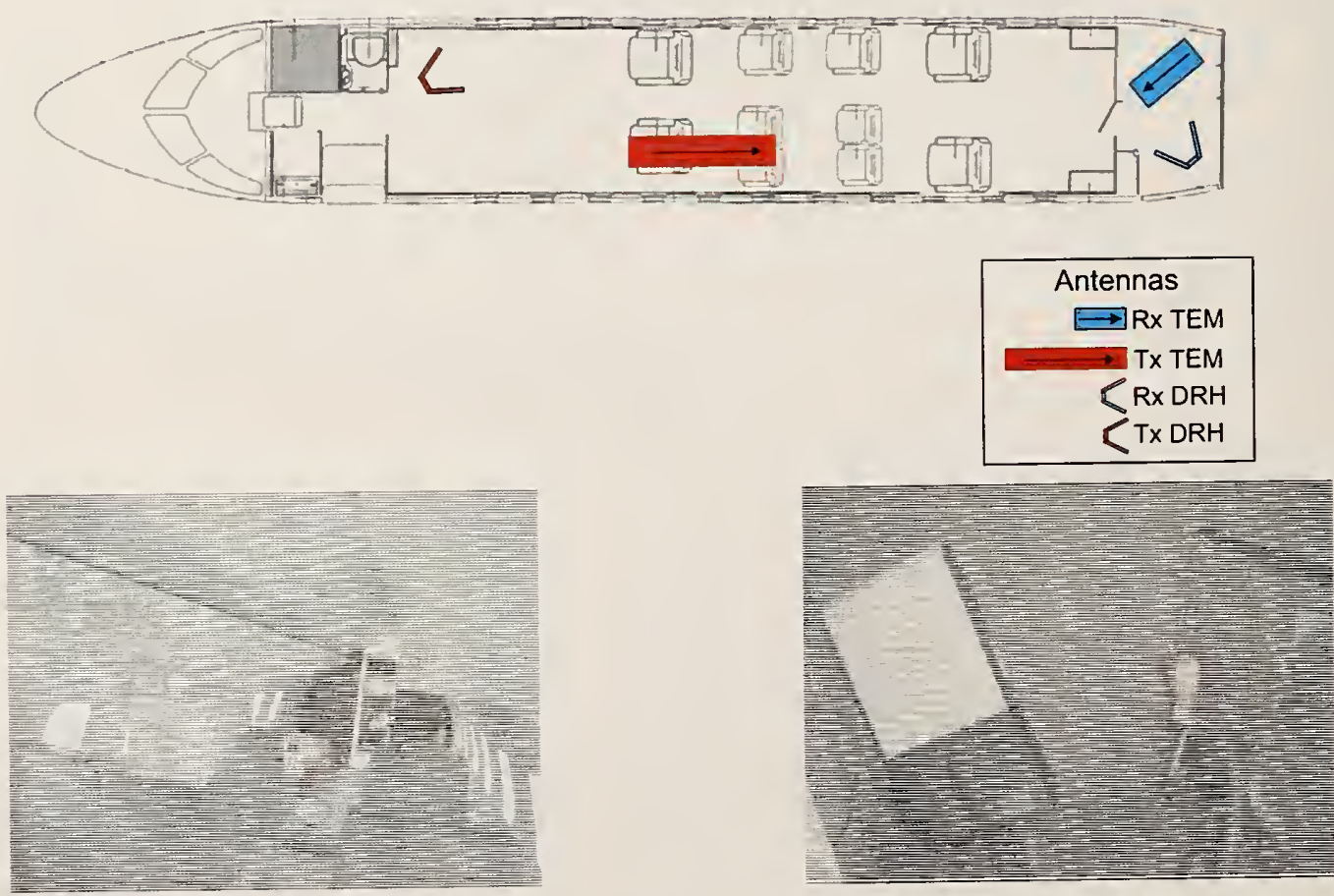

Figure 127. Layout for Configuration 1 measurements: coupling from main passenger cabin to cargo closet. 


\section{Configuration 2}
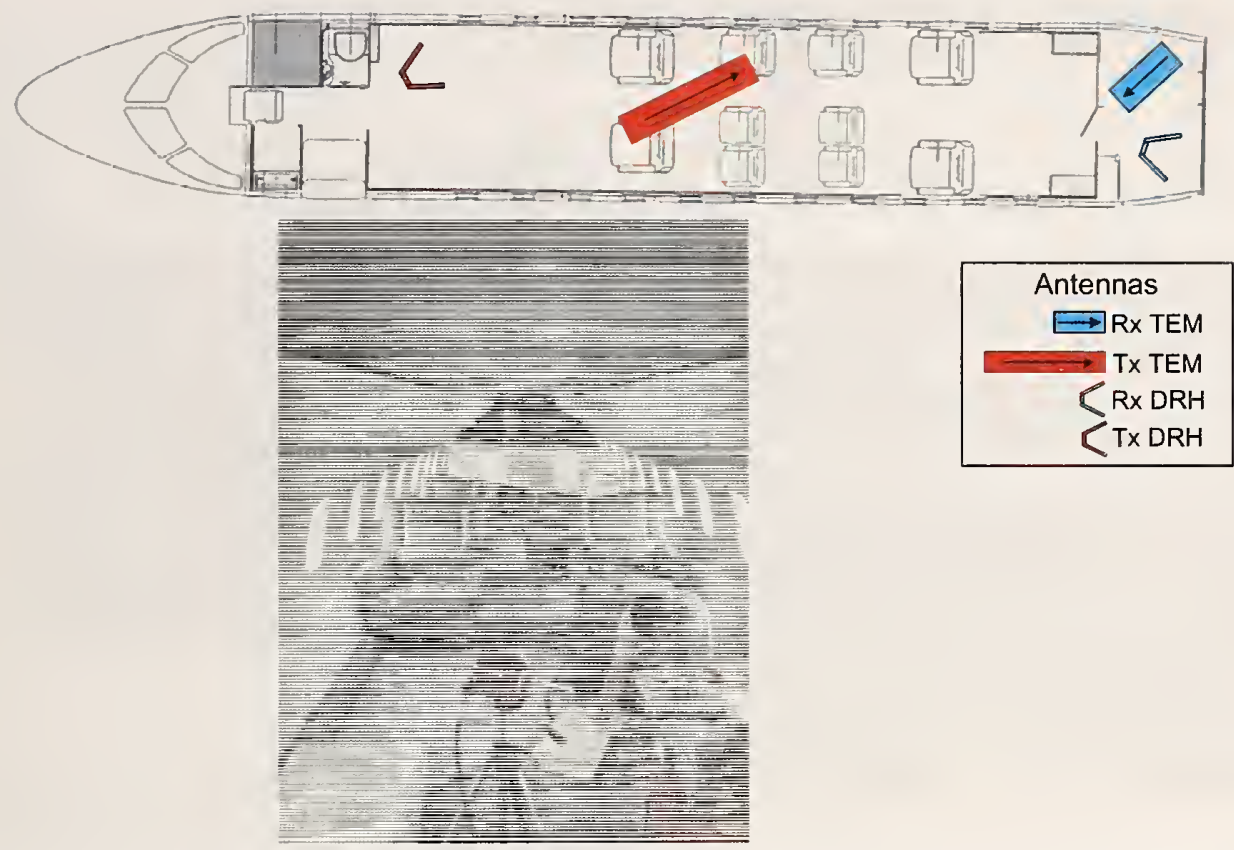

Figure 128. Layout for Configuration 2 measurements: coupling from main passenger cabin to cargo closet.

\section{Configuration 3}

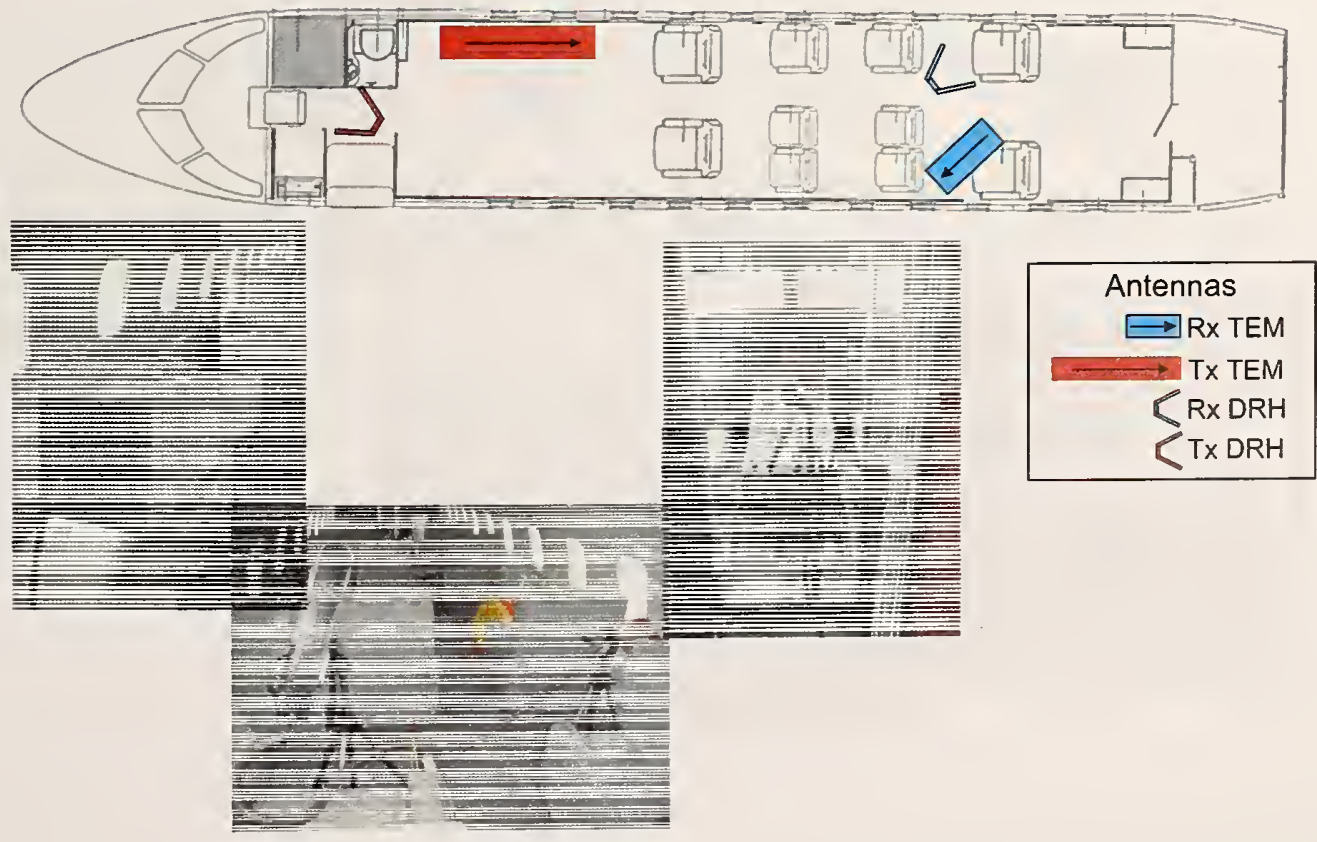

Figure 129. Layout for Configuration 3 measurements: coupling from main passenger cabin to flight deck. 


\section{Configuration 4}
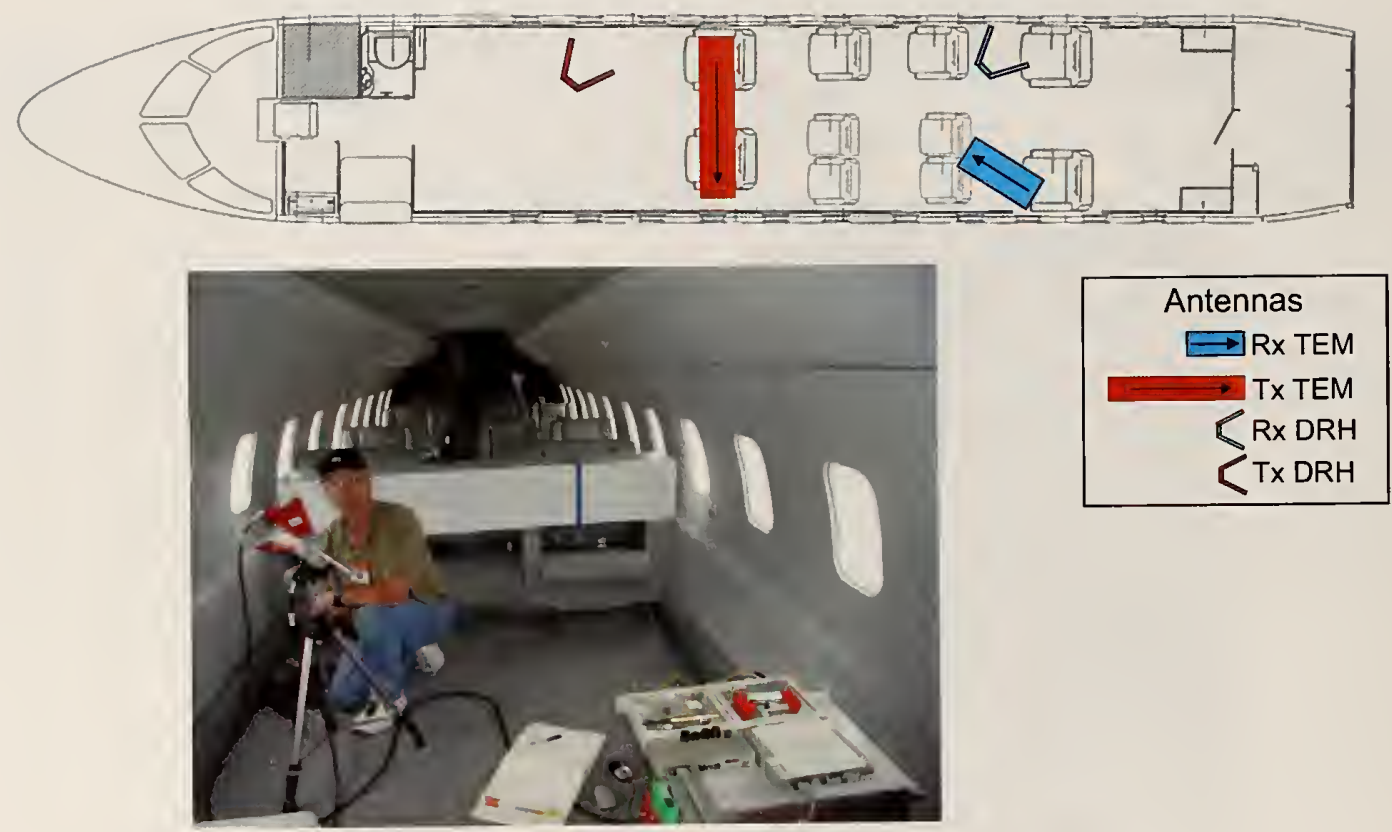

Figure 130. Layout for Configuration 4 measurements: coupling from forward main passenger cabin to aft main passenger cabin.

\section{Configuration 5}
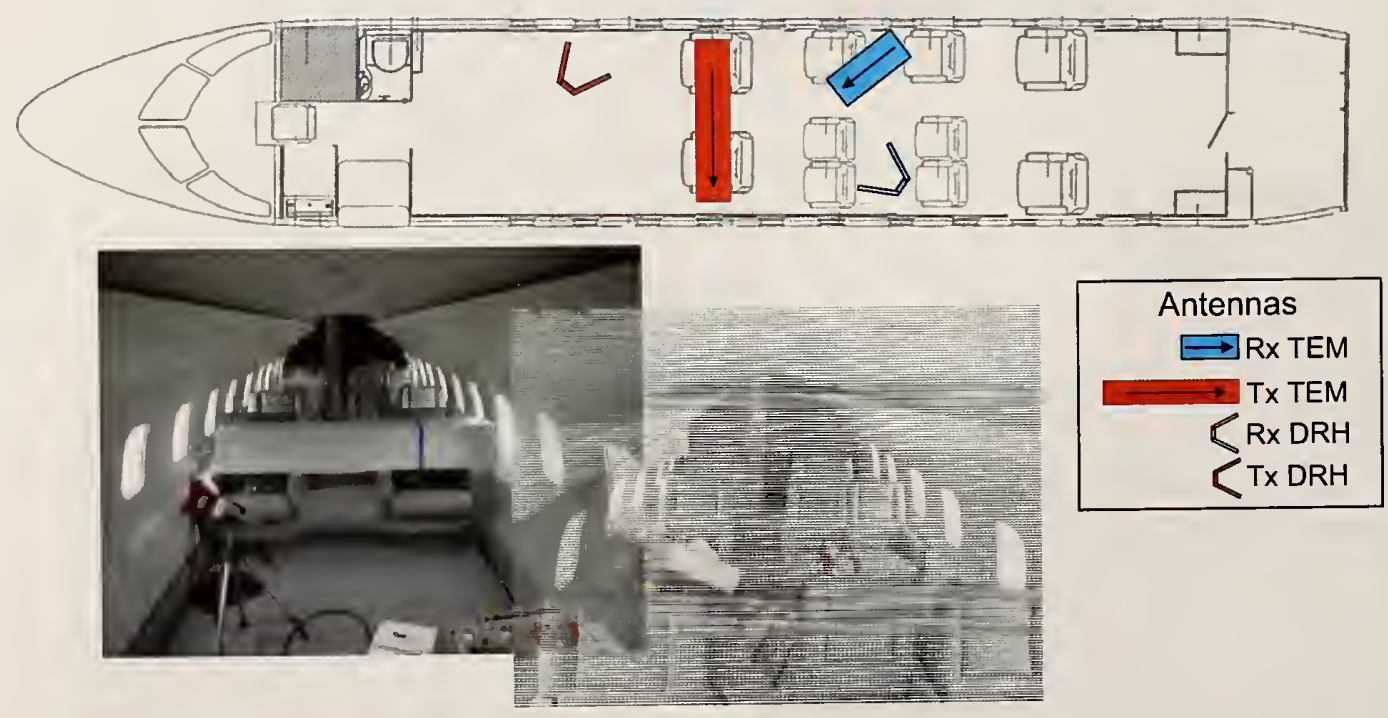

Figure 131. Layout for Configuration 5 measurements: coupling from forward main passenger cabin to mid main passenger cabin: 


\section{Configuration 6}
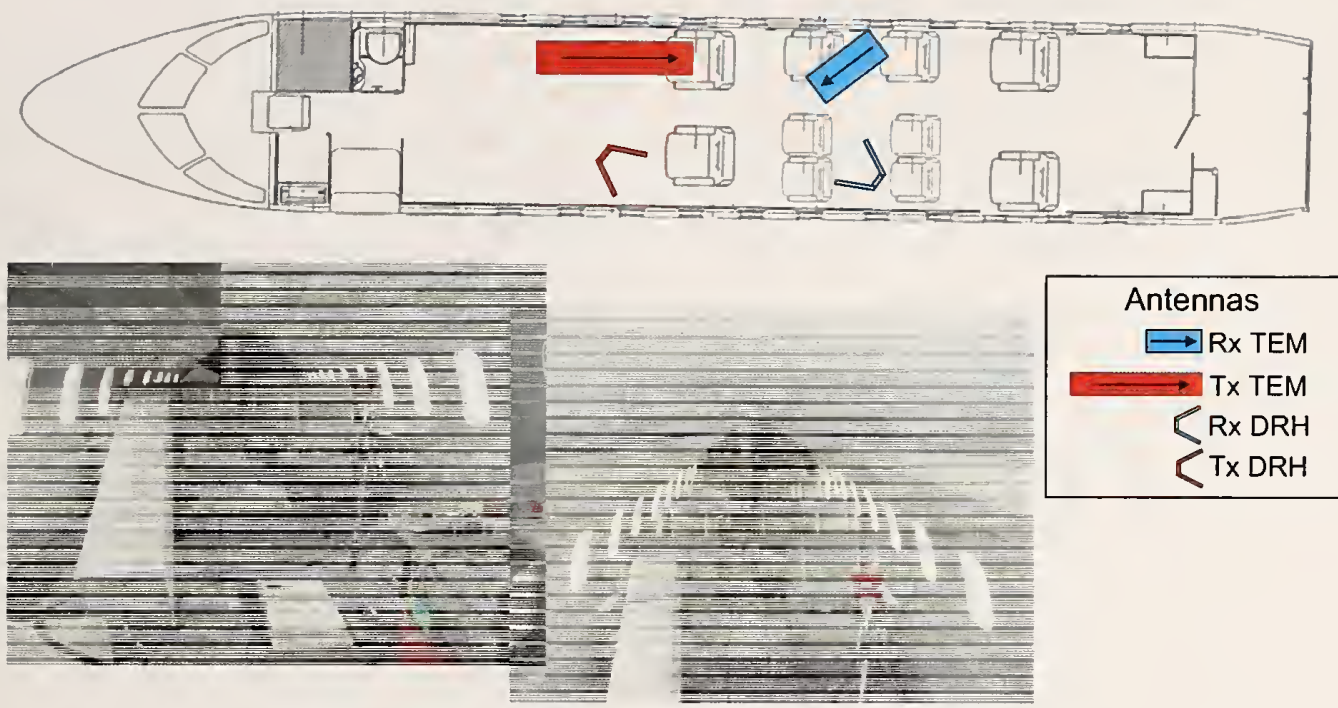

Figure 132. Layout for Configuration 6 measurements: coupling from forward main passenger cabin to mid main passenger cabin.

We began by looking at the time decay and quality factor of the airframe for each set of antennas in each configuration. These data are shown in Figures 133 to 136. At approximately $8 \mathrm{GHz}$, the time decay for the DRG antennas in Figure 133 shows a rapid departure from the clustering behavior. Below $8 \mathrm{GHz}$, the variation in time decay is anywhere from $2 \mathrm{~ns}$ to approximately 7 ns. The variation above $8 \mathrm{GHz}$ is from $7 \mathrm{~ns}$ to $10 \mathrm{~ns}$. There is no discernable pattern behavior as a function of position. The variation in time decay for the TEM horn antennas is on the order of $2 \mathrm{~ns}$ to approximately $7 \mathrm{~ns}$. The trend shows tighter clustering for the DRG antennas at the lower frequencies and for the TEM antennas in the higher frequency range. Because of the lower frequency range, the quality factor is much lower for the TEM horn antennas. Figures 137 and 138 are plots showing the combined quality factor for both antennas for Configuration 1 and Configuration 6 . The two antennas types show good agreement across their common frequency band from 1 to $2 \mathrm{GHz}$. In a reverberant cavity, we expect the quality factor and time decay to vary little throughout the cavity. However, further statistical analysis would have to be completed in order to determine that the cavity was not reverberant. 


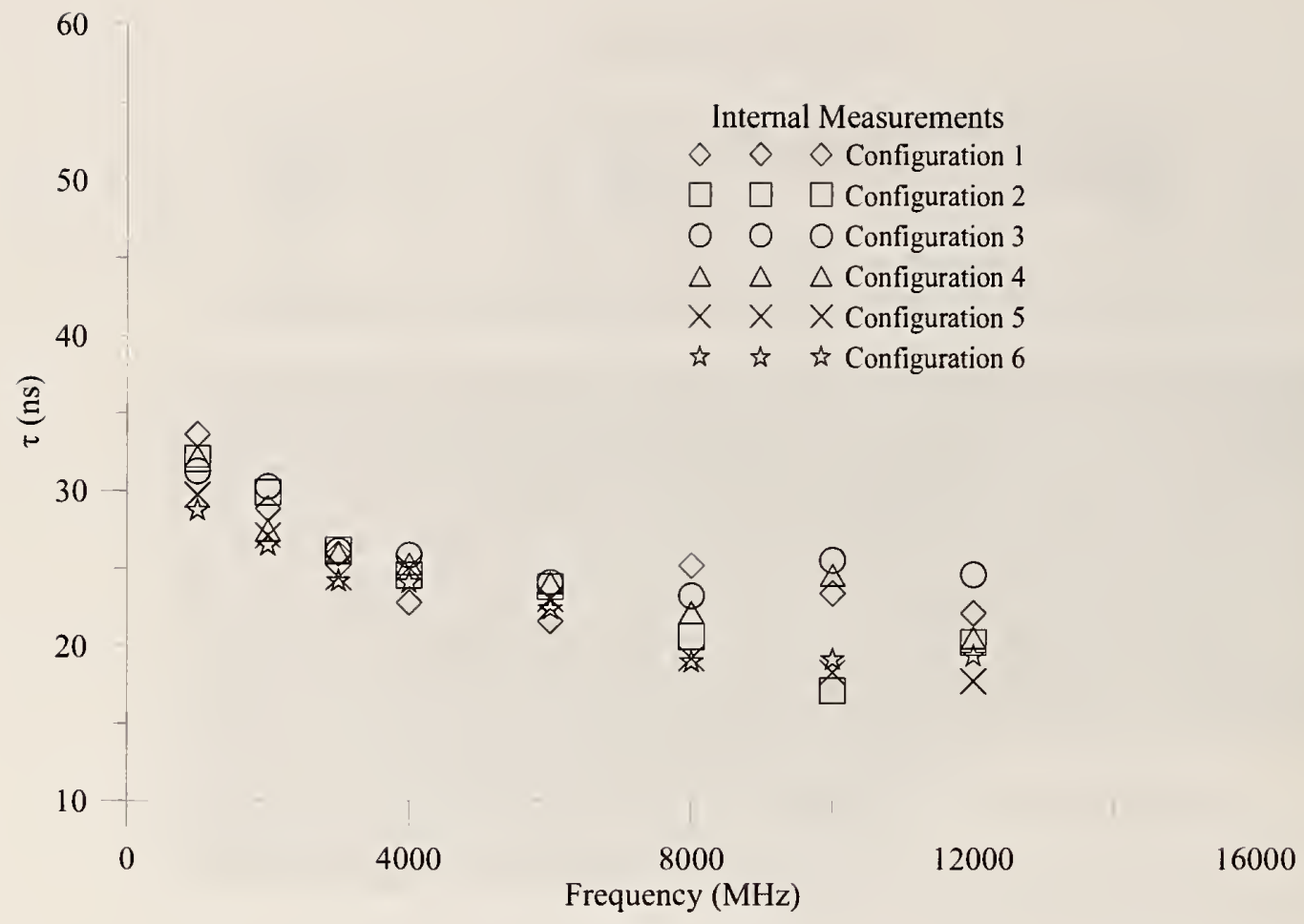

Figure 133. Time decay for DRG antennas in all six internal configurations.

2000
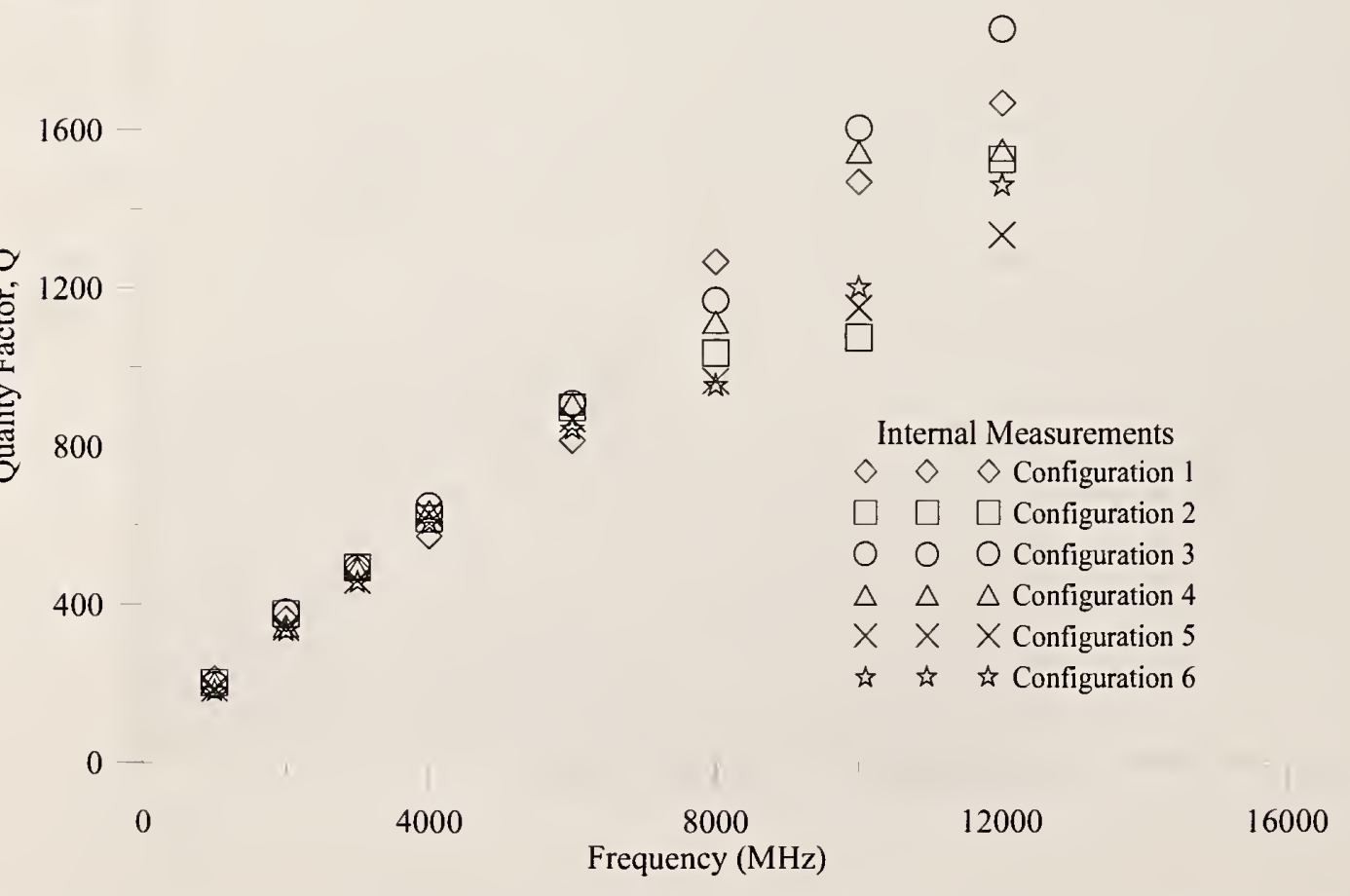

Figure 134. Airframe quality factor (Q) for DRG antennas in all six internal configurations. 


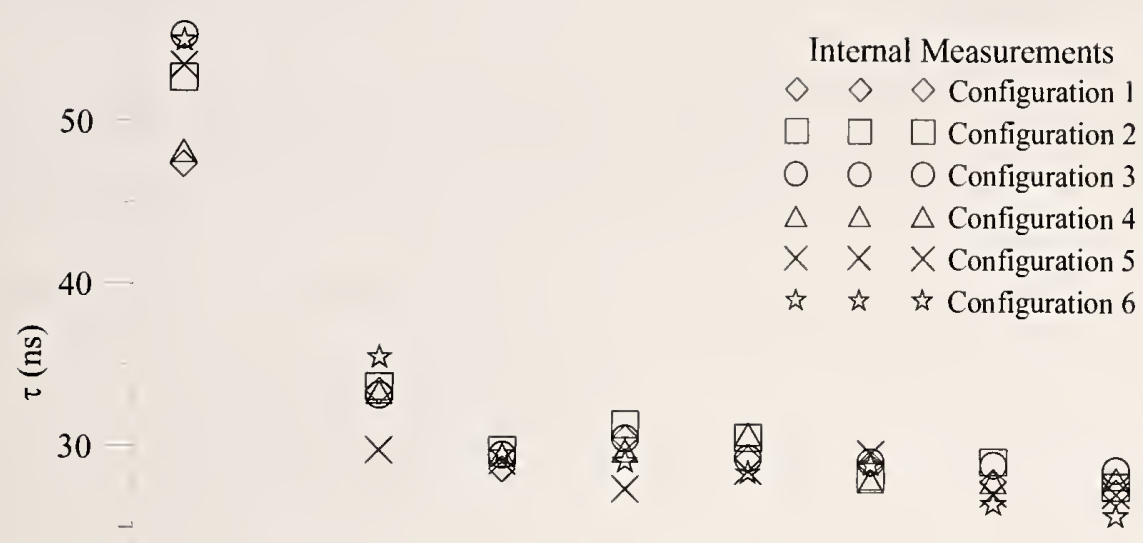

20

10

0

400

$800 \begin{array}{lc}1200 \\ & \text { Frequency }(\mathrm{MHz})\end{array}$

$1600 \quad 2000$

2400

Figure 135. Time decay for TEM horn antennas in all six internal configurations.

400

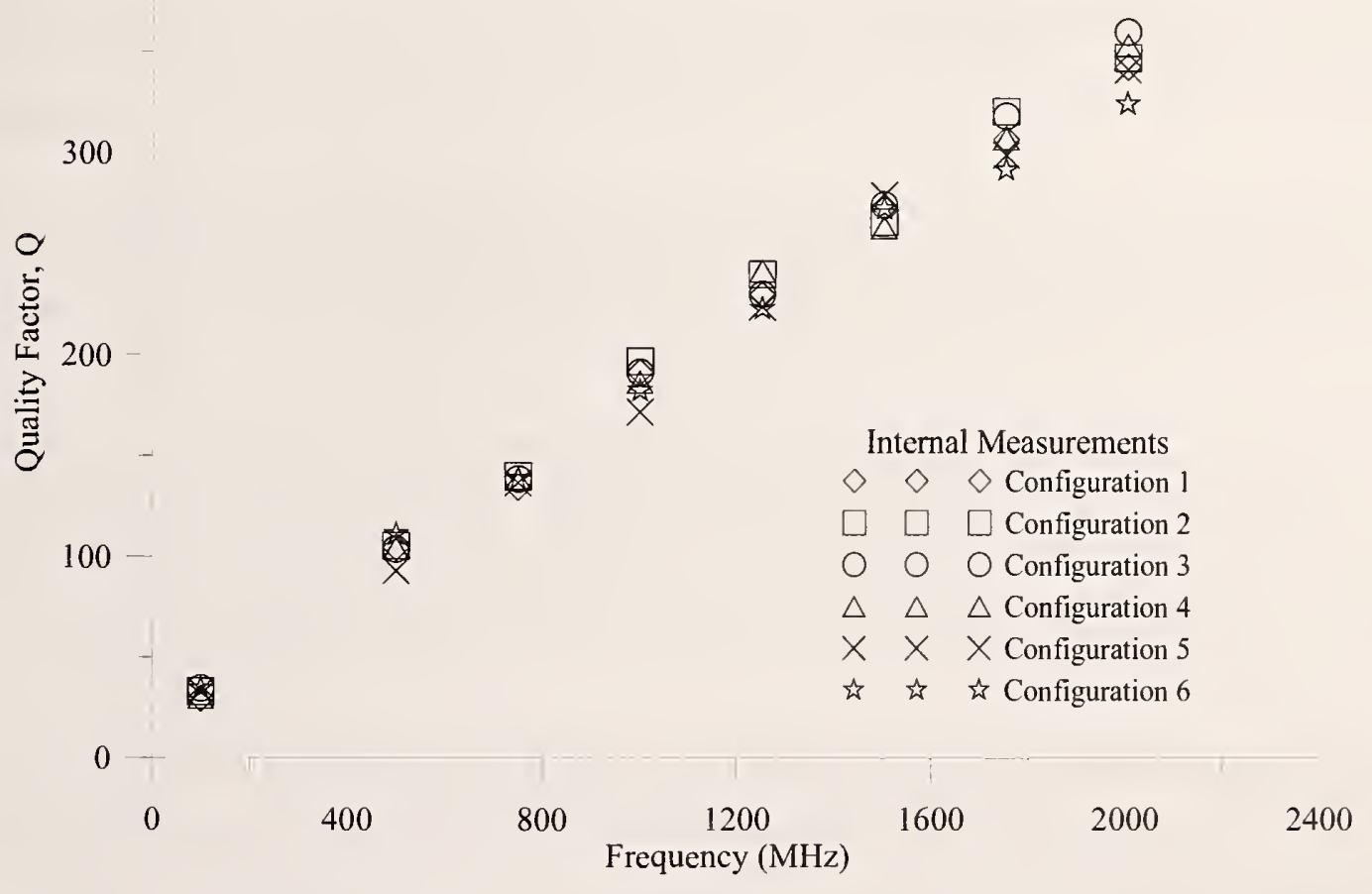

Figure 136. Airframe quality factor (Q) for TEM horn antennas in all six internal configurations. 
$1600 \quad \diamond \diamond \diamond$ Internal Measurements (DRG \& TLM)

$$
1400
$$

1200

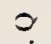

$\stackrel{\grave{\Xi}}{\grave{z}} 1000$

咅 800

600

$4(x)$

200

()

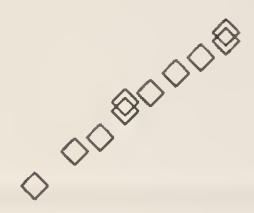

0

$+000$

Frequency $(\mathrm{NIH} \%)$

8000

12000

Figure 137. Quality factor for both antennas for Configuration 1.

1800

1600

Internal Measurements (DRG \& TEM)

$\diamond \diamond \diamond$ Configuration 6

1400

1200

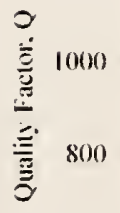

600

400

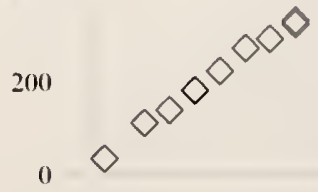

0

4000

Frequency (MIl/)

8000

12000

Figure 138. Quality factor for both antennas for Configuration 6. 


\section{Uncertainty Analysis}

The sources of uncertainty are based on NISTIR 5019 [6] which provides a natural framework for the identification of source uncertainties encountered in aircraft shielding measurements, along with an efficient method for tracking and combining them.

Six sources of measurement uncertainty are considered in our analysis:
- $\quad$ Measurement Repeatability
- $\quad$ Range Uncertainties/Distance Correction
- $\quad$ Time Gating
- Drift
- Polarization Mismatch
- $\quad$ Signal-to-Noise Ratio

These sources of uncertainties (influence factors) were evaluated as follows:

1. Measurement Repeatability: For a given internal orientation, we took multiple data sets, in which we moved the external antennas to and from the same location.

2. Range Uncertainties: We used estimates of variations when positioning the antennas for both the reference and airframe measurements.

3. Time Gating: We determined time-gating uncertainties by varying the gate width and performing a statistical analysis on the resulting amplitude spectra. See Appendix A for further discussion.

4. Drift: The uncertainty due to instrumentation drift was small and was computed by using the data obtained from system calibration checks done before and after circumnavigating the aircraft. The uncertainty was determined by looking at the difference and dividing that by the average of the two signals.

5. Polarization Mismatch: Polarization mismatch uncertainties occur because antennas could be misaligned for a given polarization. We calculated one uncertainty based on a possible misalignment of $5^{\circ}$. The other term comes from the possible leakage from one polarization to the other. The cross-polarization of our TEM horn antennas is approximately $-20 \mathrm{~dB}$, and for the DRG antennas it is approximately $-15 \mathrm{~dB}$. These two factors both contribute to the polarization mismatch uncertainty.

6. Signal-to-Noise Ratio: These uncertainties were calculated for the Global 5000 aircraft. Appendix B of this report summarizes typical signal and noise characteristics.

The impact of these six influence factors on the measured penetration is summarized in Table 1. This table lists the fractional uncertainties $[7,8]$ resulting from each influence factor. The most 
significant error is due to signal-to-noise uncertainties. Measurement repeatability has an influence that is somewhat less than the signal-to-noise ratio, but is nevertheless significant. Time gating is the next largest uncertainty, followed by the range/distance correction uncertainties, extrapolation uncertainties, polarization mismatch, and drift, respectively. Polarization mismatch and drift contribute almost nothing to the overall uncertainty.

The combined standard uncertainty was computed using the procedure given in NIST Technical Note 1297 [8]. The six sources of uncertainties were combined using the root sum-of-squares of the linear fractional uncertainties averaged over a $200 \mathrm{MHz}$ frequency window. The resulting uncertainties (in decibels) for penetration are plotted in Figure 139 for the frequency range from $800 \mathrm{MHz}$ to $1500 \mathrm{MHz}$. The uncertainties were calculated as a function of frequency, and therefore, we have listed an approximate average value in the table below. We have broken the uncertainties into those below $9 \mathrm{GHz}$ and those above $9 \mathrm{GHz}$ because the noise floor resets itself there due the internal configuration of both attenuators and mixers. We believe that a reasonable uncertainty bound would be at the $95 \%$ level for the data shown in Figure 139, remembering that this current uncertainty analysis is based on a limited set of data.

Table 1. Typical fractional uncertainties resulting from the six influence factors

\begin{tabular}{c|c|c}
\hline \multirow{2}{*}{ Influence factor } & \multicolumn{2}{|c}{ Typical fractional uncertainties $(\mathrm{dB})$} \\
\cline { 2 - 3 } & $<9 \mathrm{GHz}$ & $>9 \mathrm{GHz}$ \\
\hline Signal-to-noise & 1.00 & 1.20 \\
\hline Repeatability & 0.60 & 0.80 \\
\hline Time gating & 0.03 & 0.20 \\
\hline Range/distance correction & 0.28 & 0.28 \\
\hline Extrapolation & 0.15 & 0.40 \\
\hline Polarization mismatch & 0.06 & 0.06 \\
\hline Drift & 0.01 & 0.01 \\
\hline RSS & 1.82 & 4.06 \\
\hline
\end{tabular}




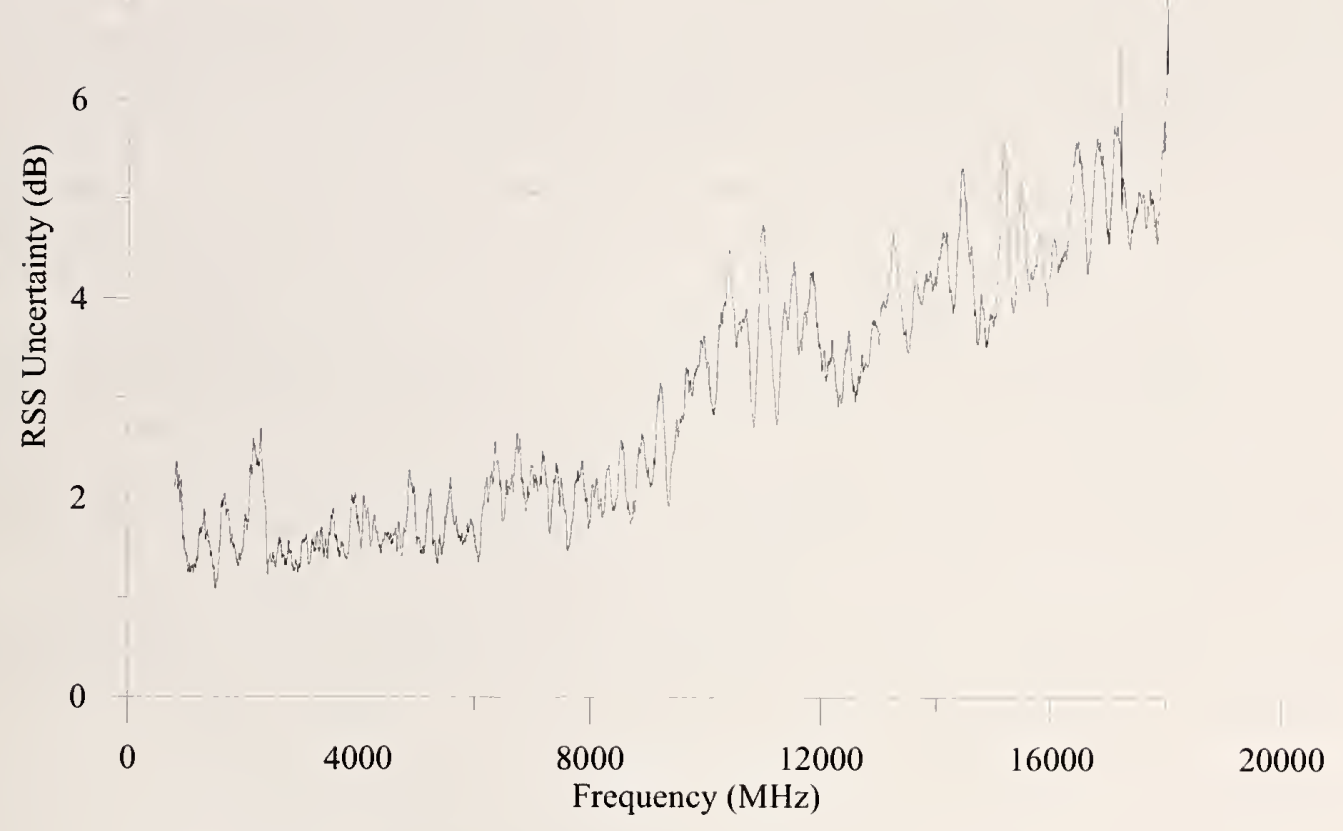

Figure 139. Combined standard uncertainty in penetration.

\section{Summary and Conclusions}

The data collected in this report will be used to provide guidelines for HIRF testing on various aircraft. We have reported on the penetration measurements taken on the Bombardier Global 5000 at the FAA facility in Atlantic City, New Jersey. We have discussed extrapolation measurements taken to ensure that the measurements are in the far-field of the transmitting antenna. We have shown results for measurements taken at various azimuth angles around the outside of the aircraft with the receiving antenna in the main passenger cabin, the flight deck, and the cargo closet. We have also performed a series of internal measurements to look at the reverberant field characteristics within the cavity to determine the viable statistics associated with the fields.

The main passenger cabin shows the greatest penetration for all three compartments. We attribute this to the numerous windows. Typical maximum penetration values are $0 \mathrm{~dB}$ and appear at angles between $90^{\circ}$ and $150^{\circ}$. Minimum penetration values occur at the front and rear of the aircraft and are on the order of $-40 \mathrm{~dB}$. Flight deck maximum penetration is around -15 $\mathrm{dB}$, occurring around $45^{\circ}$, and minimum penetration values are around $-45 \mathrm{~dB}$, occurring at $190^{\circ}$. Cargo closet penetration values are similar to the flight deck numbers. Maximum penetration values occur at $30^{\circ}, 60^{\circ}$, and $330^{\circ}$, while minimum penetration occurs at $0^{\circ}, 180^{\circ}$, 
and $350^{\circ}$. We also found that the horizontal polarization has higher penetration values than the vertical polarization, and that symmetry is maintained around the aircraft until we get to the main passenger door, and then values increase slightly. HIRF penetration into the flight deck shows approximate values of $-20 \mathrm{~dB}$ at lower frequencies and $-25 \mathrm{~dB}$ at higher frequencies for a radial distance of $15 \mathrm{~m}$ from the front landing gear at angles of $270^{\circ}, 315^{\circ}, 0^{\circ}, 45^{\circ}$, and $90^{\circ}$. The internal coupling measurements showed that traditional reverberation statistics would not be valid in this cavity, but good agreement was shown between the two antenna types for quality factor values between $1 \mathrm{GHz}$ and $2 \mathrm{GHz}$. Finally, we found that major coupling apertures would not be missed if measurements were taken every $10^{\circ}$ as opposed to every $5^{\circ}$. Figure 140 shows penetration data measured with the DRG horn antenna in both the vertical and horizontal polarizations for various frequencies in each compartment. Figure 141 shows penetration data measured with the TEM horn antenna in both the vertical and horizontal polarizations for various frequencies in each compartment. Red circles are in the main cabin, blue triangles are in the flight deck, and green squares are in the cargo closet. This shows us that flight deck penetration is typically lower than for the cargo closet at each of these frequencies. And we see that penetration typically decreases as frequency increases. This was an important measurement effort both to aid in understanding and to help define standard measurement procedures for HIRF testing. 


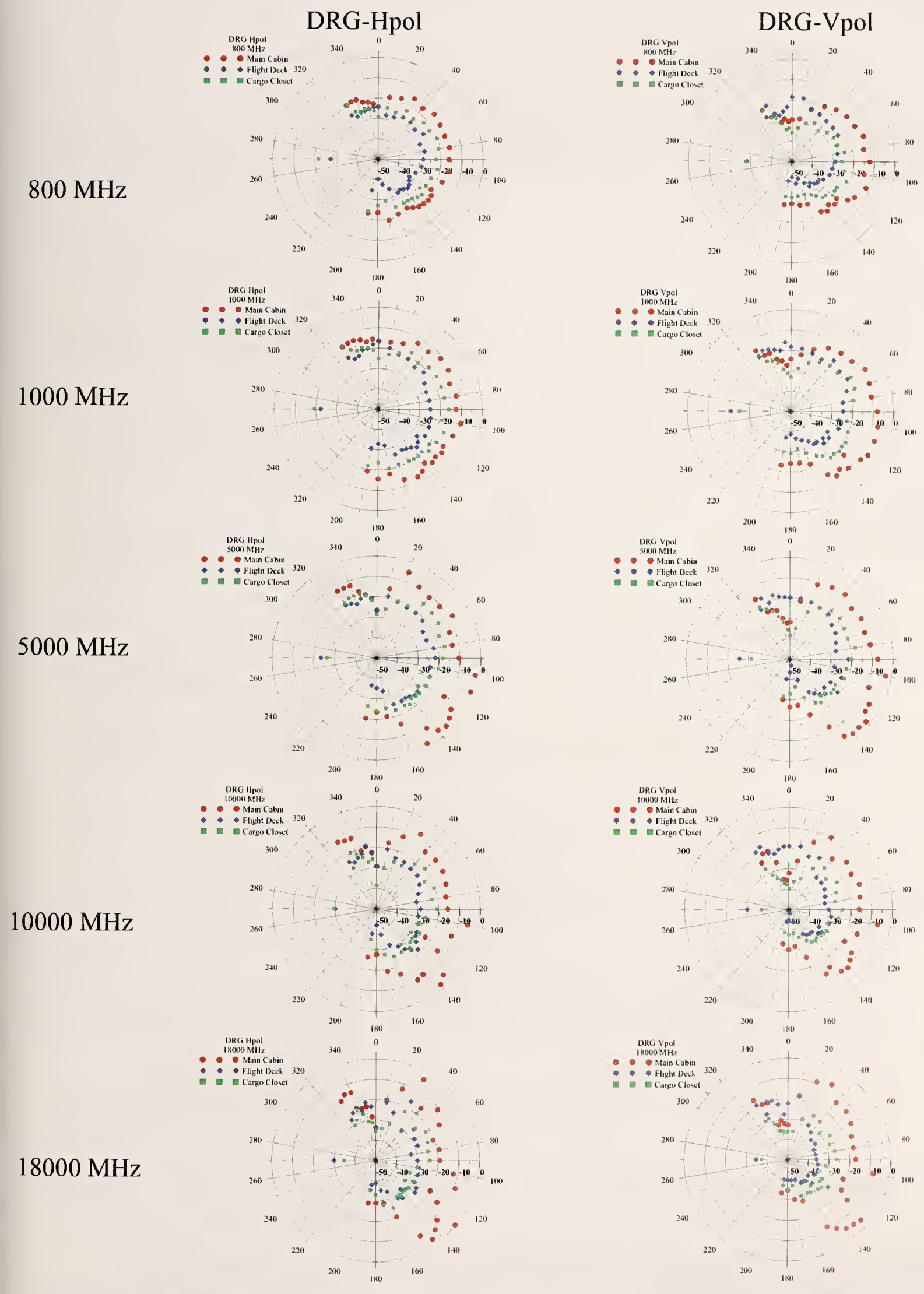

Figure 140. Penetration data as a function of angular position for DRG horn antenna in both vertical and horizontal polarizations for various frequencies in each compartment. Red circles (O) are in the main cabin, blue diamonds $(\diamond)$ are in the flight deck, and green squares $(\square)$ are in the cargo closet. 


\section{TEM-Hpol}

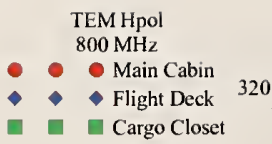

300

280$$
\text { . }
$$

(1)

280

$800 \mathrm{MHz} \quad 260$

240

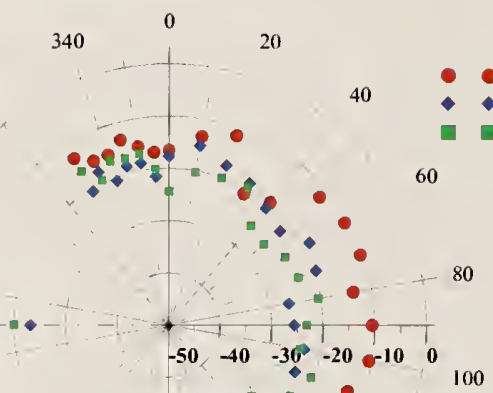

120

220

140

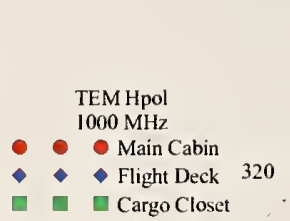

$1000 \mathrm{MHz}$

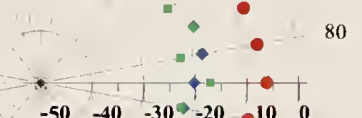

300

280

260

240

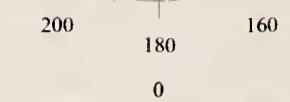

$340 \quad 0 \quad 20$

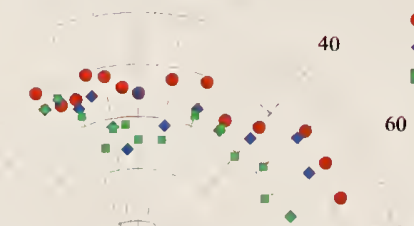

-
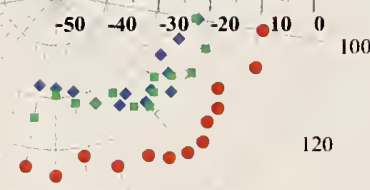

220

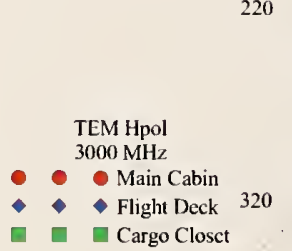

300

\section{0}

$3000 \mathrm{MHz}$

260

240

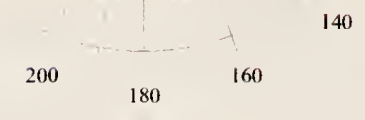

120

140

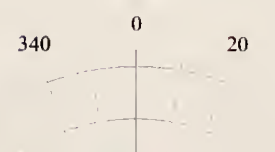

- Main Cabin

- Flight Deck 320

Cargo Closet

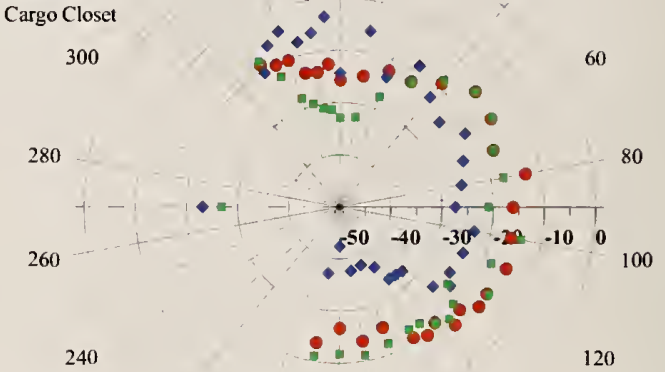

220
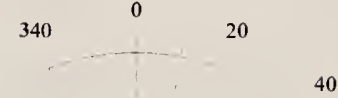

40

60

80 00 
We would like to thank Robert Morrison and Anthony Wilson from the FAA in Atlantic City, New Jersey and David Walen from the FAA office in Renton, Washington for their excellent assistance and financial support for this work.

\section{References}

[1] SAE Aerospace Group, Guide to certification of aircraft in a high intensity radiated field (HIRF) environment. SAE ARP5583 (January 2003).

[2] C. A. Grosvenor, R. T. Johnk, D. R. Novotny, N. Canales, C. M. Weil, J. Veneman, A twophase airframe shielding performance study using ultrawideband measurement systems. Natl. Inst. Stand. Technol. NISTIR 6622 (January 2003).

[3] J. M. Ladbury, T. H. Lehman, G. H. Koepke, Coupling to devices in electrically large cavities or why classical emc evaluation techniques are becoming obsolete. 2002 Intl Symp. on Electromagnetic Compatibility, 2: 648-655 (August 2002).

[4] P. F. Wilson, D. A. Hill, C. L. Holloway, On determining the maximum emissions from electrically large sources. 2002 IEEE Trans. Electromagnetic Compatibility, 44(1): 79-86 (February 2002).

[5] R. T. Johnk, B. Archambeault, D. Novotny, Using joint time-frequency analysis to enhance time-domain numerical emc simulations. 2003 Annual Report Interference Technology (2003).

[6] R. C. Wittmann, M. H. Francis, L. A. Muth, R. L. Lewis, Proposed uncertainty analysis for RCS Measurements. Natl. Inst. Stand. Technol. NISTIR 5019 (January 1994).

[7] J. R. Taylor, An introduction to error analysis. University Science Books, Sausolito, CA, (1997).

[8] B. N. Taylor, C. E. Kuyatt, Guidelines for evaluating and expressing the uncertainty of NIST measurement results. Natl. Inst. Stand. Technol. Tech. Note 1297 (September 1994).

[9] E. B. Larsen, R. L. Ehret, D. G. Camell, G. H. Koepke, Calibration of antenna factor at a ground screen field site using an automatic network analyzer. 1989 Intl. Symp on Electromagnetic Compatibility, 1: 19-24 (August 1989).

[10] R.T. Johnk; Ultrawideband shielding evaluation of a business jet. SAE Lightning Committee Meeting (May 2006). 


\section{Appendix A. Time Domain Waveform Analysis}

Time-domain waveforms provide important information on the penetration of electromagnetic fields into an airframe. Figure 142 shows the time-domain waveform for a DRG antenna transmitting in the horizontal polarization at an angle of $70^{\circ}$. The waveform shows an initial direct antenna response followed by a short period where the signal decays, and then a secondary impulse that decays into the reverberant fields inside the airframe. These different waveform characteristics are outlined in Figure 143. We have defined the four parts of the waveform as follows: (1) the direct component, shown in the black rectangle, includes only the initial impulse response from the antenna; (2) the initial gate, shown in the blue rectangle, includes the direct component plus the decay from this initial impulse; (3) the reverberant component, shown in the green rectangle, includes the secondary signal build-up and the signal decay out to where the energy of the signal no longer contributes significantly to the signal; and (4) the entire signal, shown in the red rectangle, which extends from the initial part of the waveform until the same point in (3) where the energy no longer contributes significantly to the signal. From the waveform in Figure 143 we see that the direct component comprises a small portion of the entire signal, so we expect that this component will not contribute much to the airframe penetration.

The initial gate, which includes the direct component, contributes a little more to the entire signal but will not contribute much to overall penetration. In this example, the reverberant fields contribute the most energy to the entire signal. We conclude that the reverberant fields at this position are very important to the airframe penetration. This is shown by the frequency response curves in Figure 144. Note that the entire signal waveform (direct + reverberant parts) looks very similar to the reverberant signal waveform.

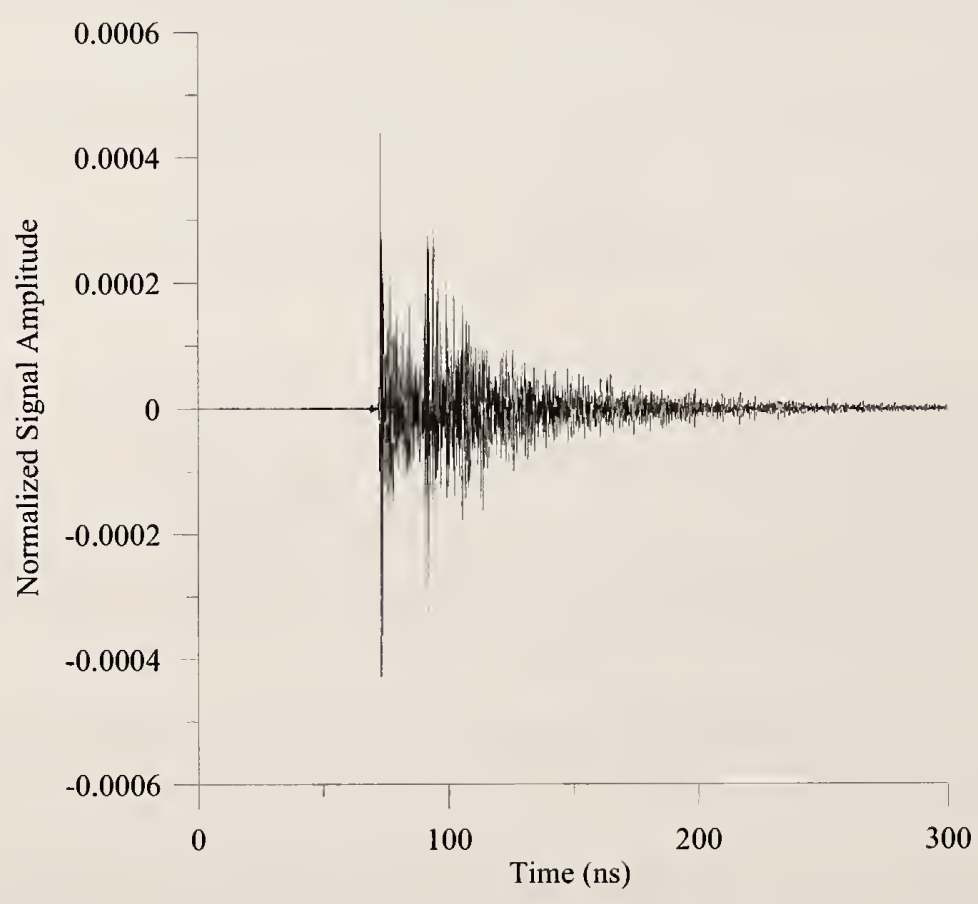

Figure 142. Time-domain waveform for a DRG antenna transmitting at angular position $70^{\circ}$. Receiving antenna in the main passenger cabin. 
Let's examine another position, at $140^{\circ}$, where the direct component is large compared to the reverberant fields. This is shown in Figure 145 using the same color scheme given above. Here we see that the direct component is almost five times larger than that for Figure 143, and so we expect both the direct component and the initial gate to contribute more to the overall penetration into the aircraft and for the reverberant fields to contribute significantly less. Figure 146 is the frequency-domain response for this same position, showing that indeed the direct component and initial gate contribute strongly to the overall penetration and the reverberant fields contribute very little. Notice also that the direct component is a smoothed version of the entire signal, and that the initial gate, which is a combination of the direct response and the initial decay of the direct response, shows much of the same "hashy" behavior of the entire signal. This tells us that the noise associated with this signal is a direct result of secondary reflections. We saw this same phenomenon when we gated out the ground bounce from our reference measurements in Section 4. Finally, at the $180^{\circ}$ position, where no direct component is available, we find that the signal, initial decay, and reverberant fields seem to contribute equally to the penetration curves shown in Figures 147 and 148.

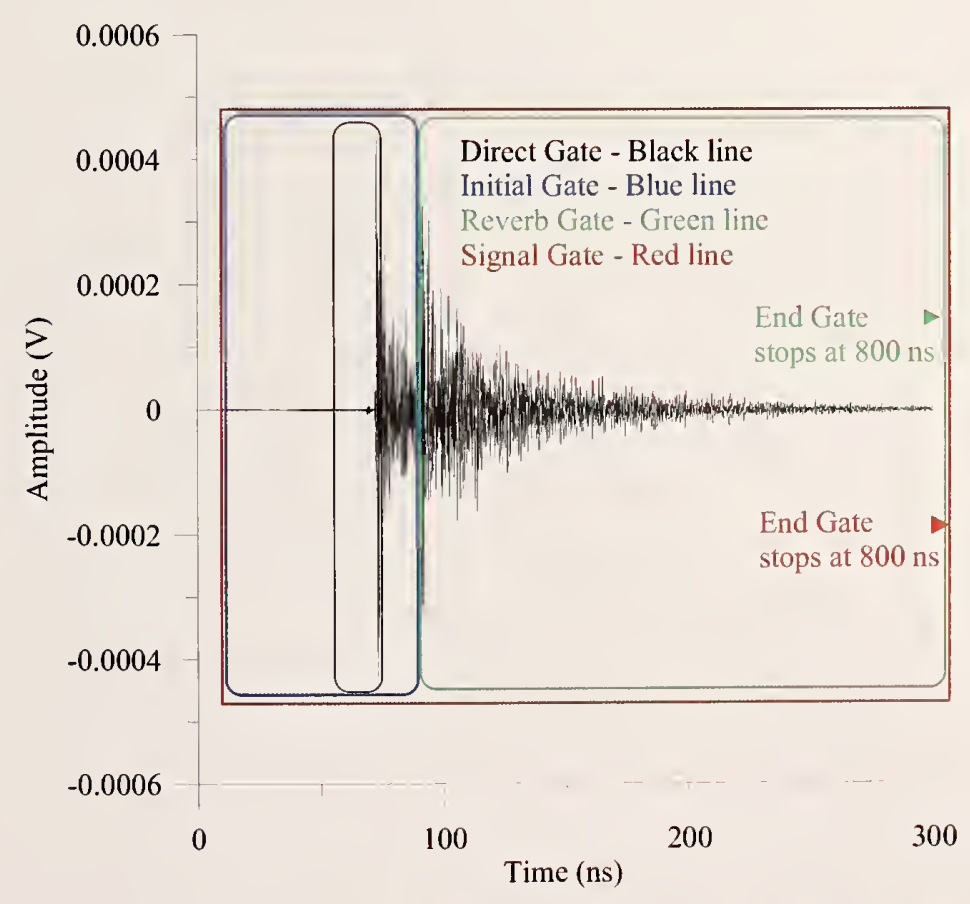

Figure 143. Time-domain waveform for a DRG antenna transmitting at angular position of $70^{\circ}$. Application of various gates to look at penetration dependence for the direct antenna interaction and reverberant fields. 


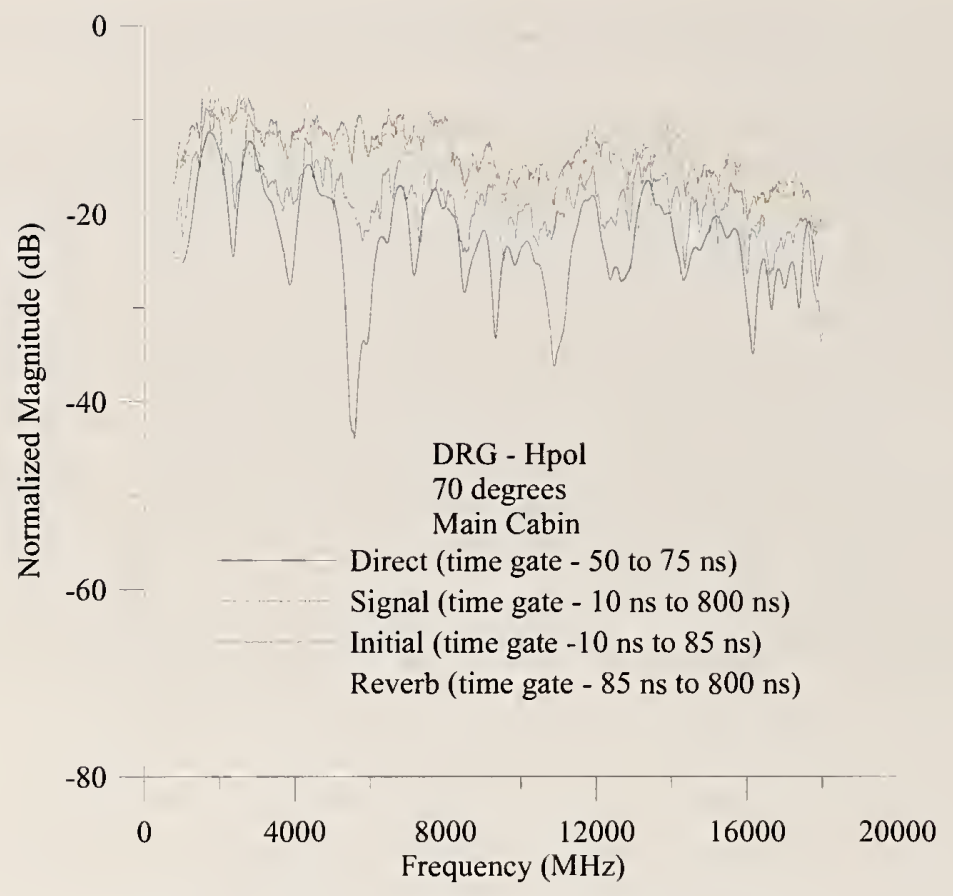

Figure 144. Frequency-domain curves for a DRG antenna transmitting at angular position of $70^{\circ}$. Application of various gates to look at contribution to penetration of the direct antenna interaction or the reverberant fields. 


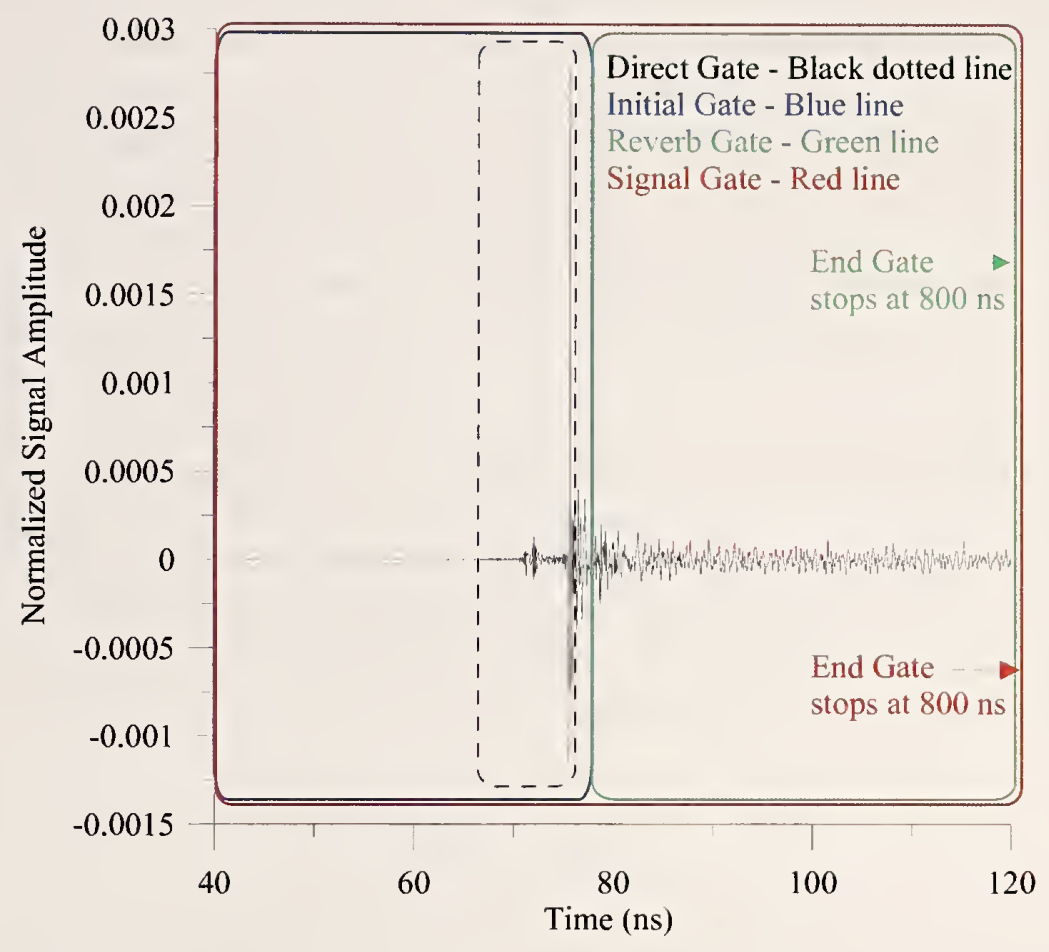

Figure 145. Time-domain waveform for a DRG antenna transmitting at angular position of $140^{\circ}$. Application of various gates to look at penetration dependence for direct antenna interaction and reverberant fields.

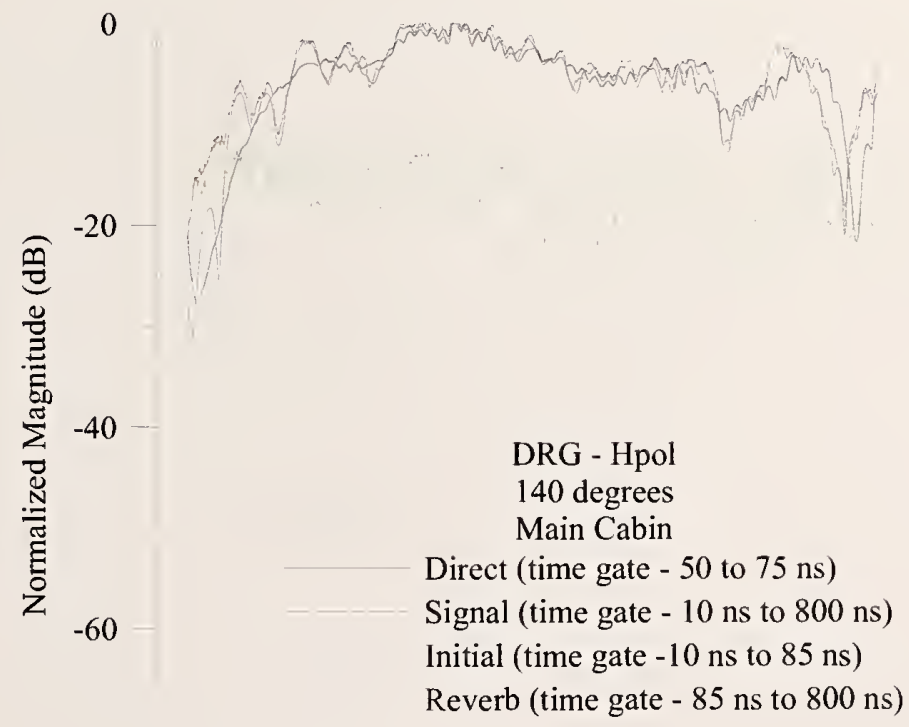

$-80$

$\begin{array}{llllll}0 & 4000 & 8000 & 12000 & 16000 & 20000\end{array}$

Frequency $(\mathrm{MHz})$

Figure 146. Frequency-domain curves for a DRG antenna transmitting at angular position of $140^{\circ}$. Application of various gates to look at contribution to penetration of the direct antenna interaction or the reverberant fields. 
After understanding how gating can affect penetration, we must answer the questions: (1) When time gating, should we keep or discard the direct components? (2) Is it better to remove the direct component and observe only the reverberation part of the waveform? and (3) If we keep the direct component to obtain penetration, how much can this bias our results? We have plotted the penetration results at several frequencies for both the reverberant components and the reverberant plus the direct components around the aircraft, as shown in Figures 149 to 158 . The data for the DRG antenna in the horizontal polarization are shown in Figure 149 to 153 and for the DRG antennas in the vertical polarization in Figures 154 to 158. The data show that including the direct component can introduce a larger error than if it is not included. This is because the direct component contains many of the higher-frequency components. For this reason, when processing the data shown in this report, we have included both the direct and reverberant components. Since no direct component was observed for the TEM horn antennas in either polarization, the answer to this question is obvious and makes the argument selfconsistent. There is less penetration at the flight deck windows and rear of the aircraft with the antenna in the main passenger cabin. More penetration is observed at positions where the transmitter has direct access to the main passenger cabin.

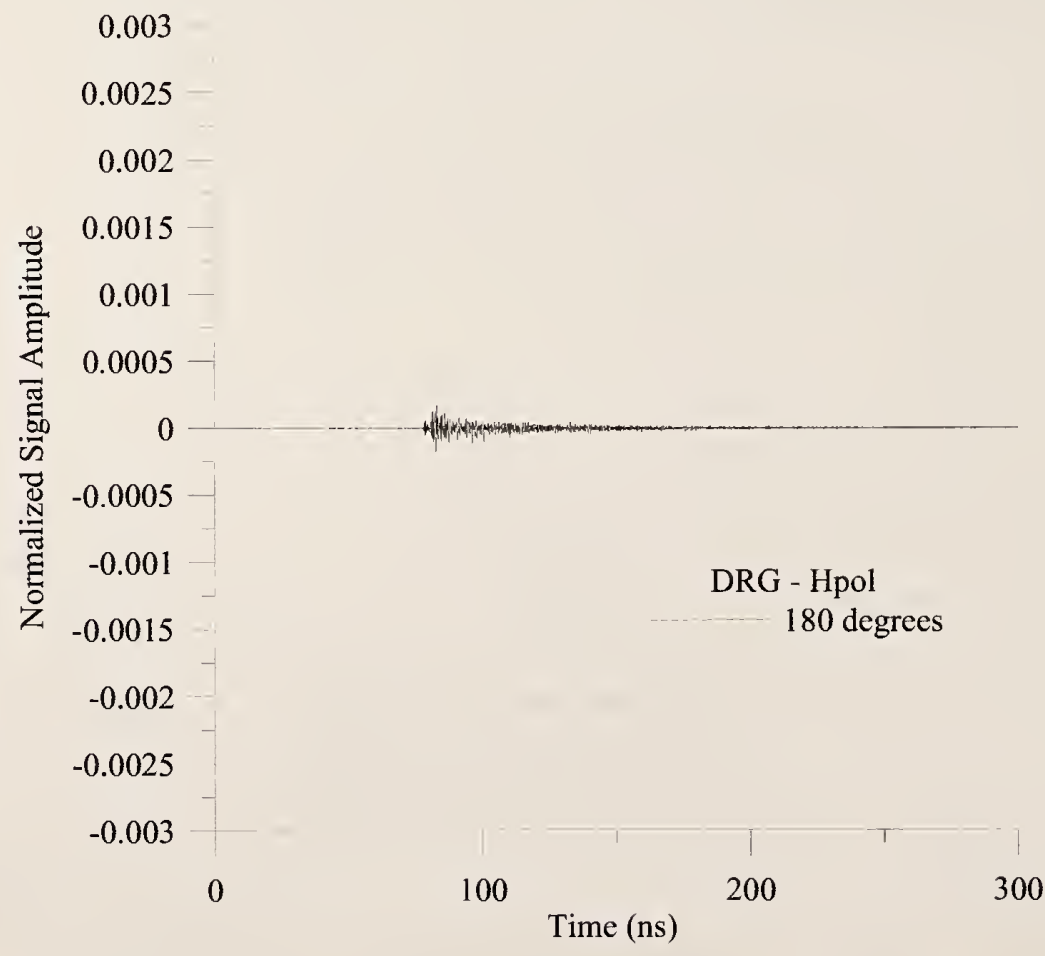

Figure 147. Time domain waveform for a DRG antenna transmitting at angular position of $180^{\circ}$. Application of various gates to look at penetration dependence for direct antenna interaction and reverberant fields. 


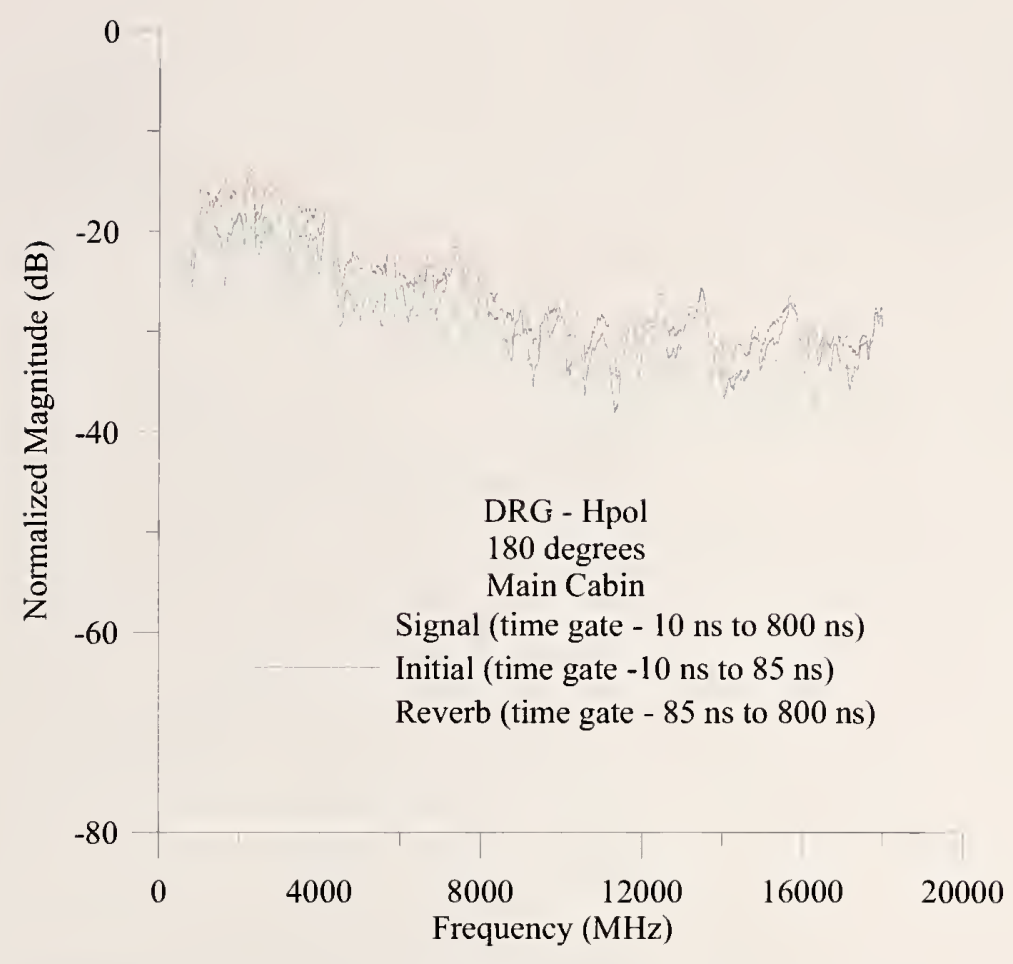

Figure 148. Frequency domain curves for a DRG antenna transmitting at angular position of $180^{\circ}$. Application of various gates to look at contribution to penetration of the direct antenna interaction or the reverberant fields. 


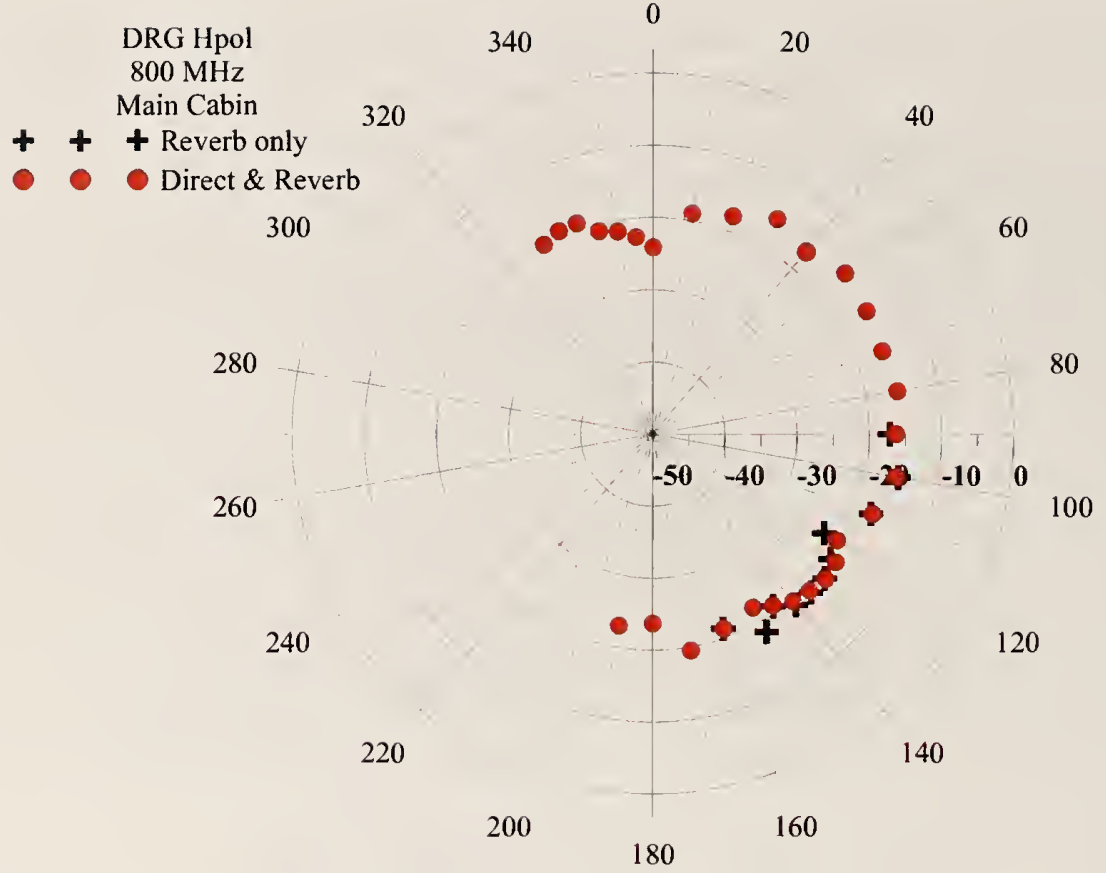

Figure 149. Penetration at $800 \mathrm{MHz}$, for the DRG antennas in the horizontal polarization, by computing only the reverberant component (black $\boldsymbol{+}$ ) vs. computing the direct plus reverberant component (red $\odot$ ).

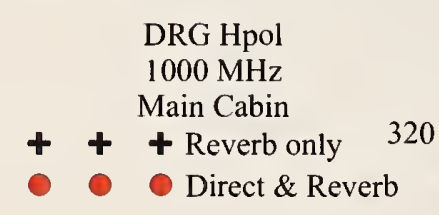

300

280

260

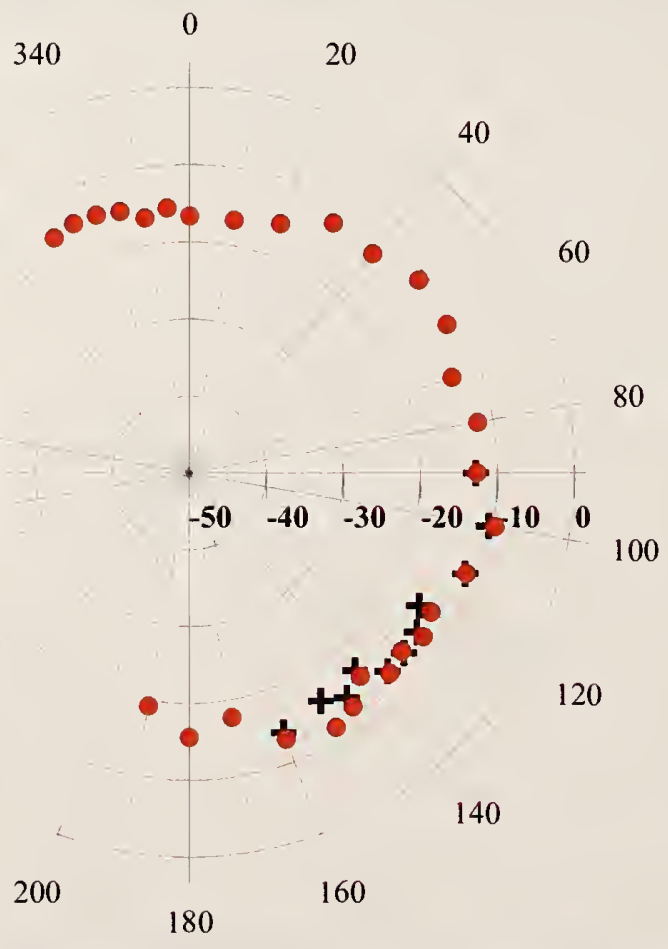

Figure 150. Penetration at $1000 \mathrm{MHz}$, for the DRG antennas in the horizontal polarization, by computing only the reverberant component (black $\boldsymbol{+}$ ) vs. computing the direct plus reverberant component (red $९$ ). 


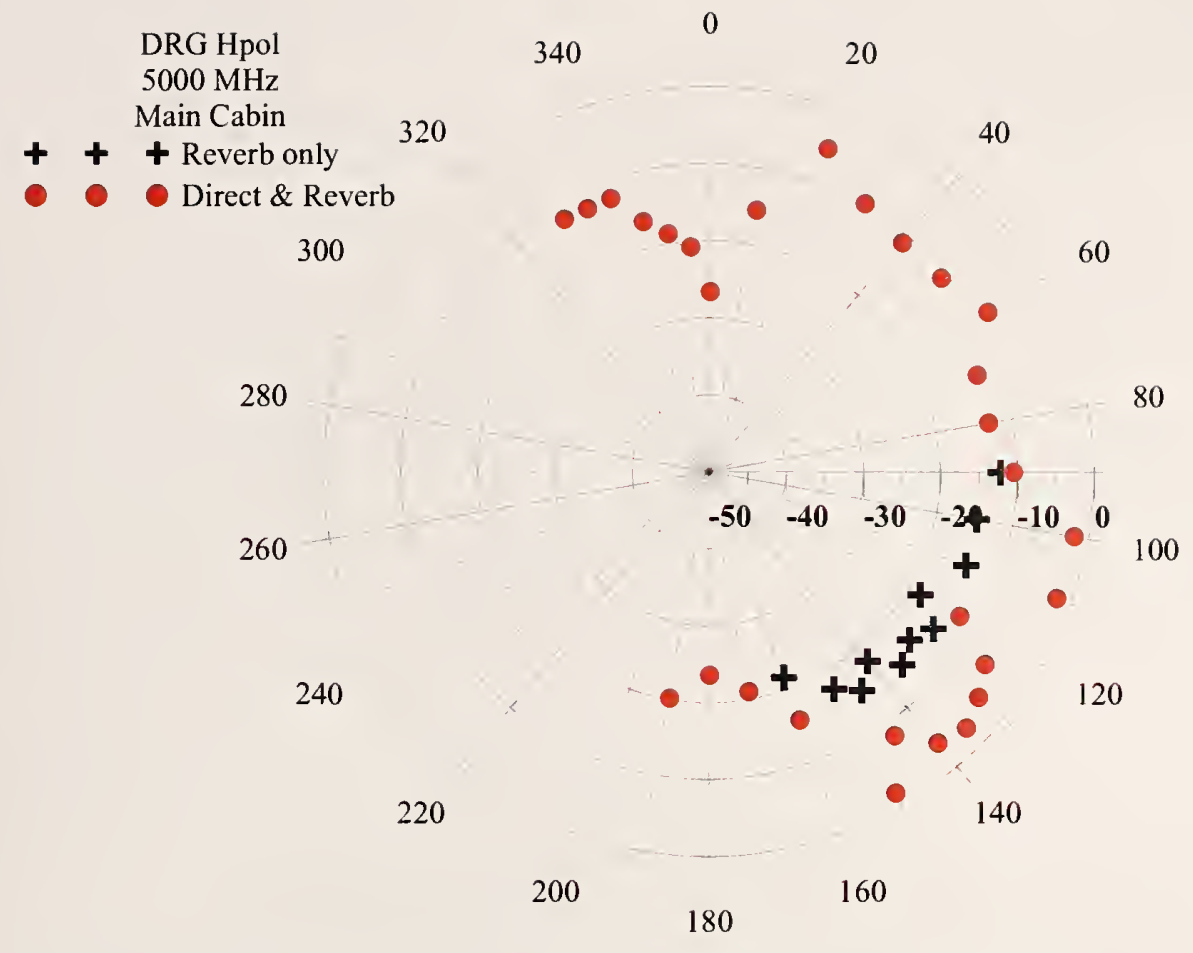

Figure 151. Penetration at $5000 \mathrm{MHz}$, for the DRG antennas in the horizontal polarization, by computing only the reverberant component (black + ) vs. computing the direct plus reverberant component (red $\odot$ ).

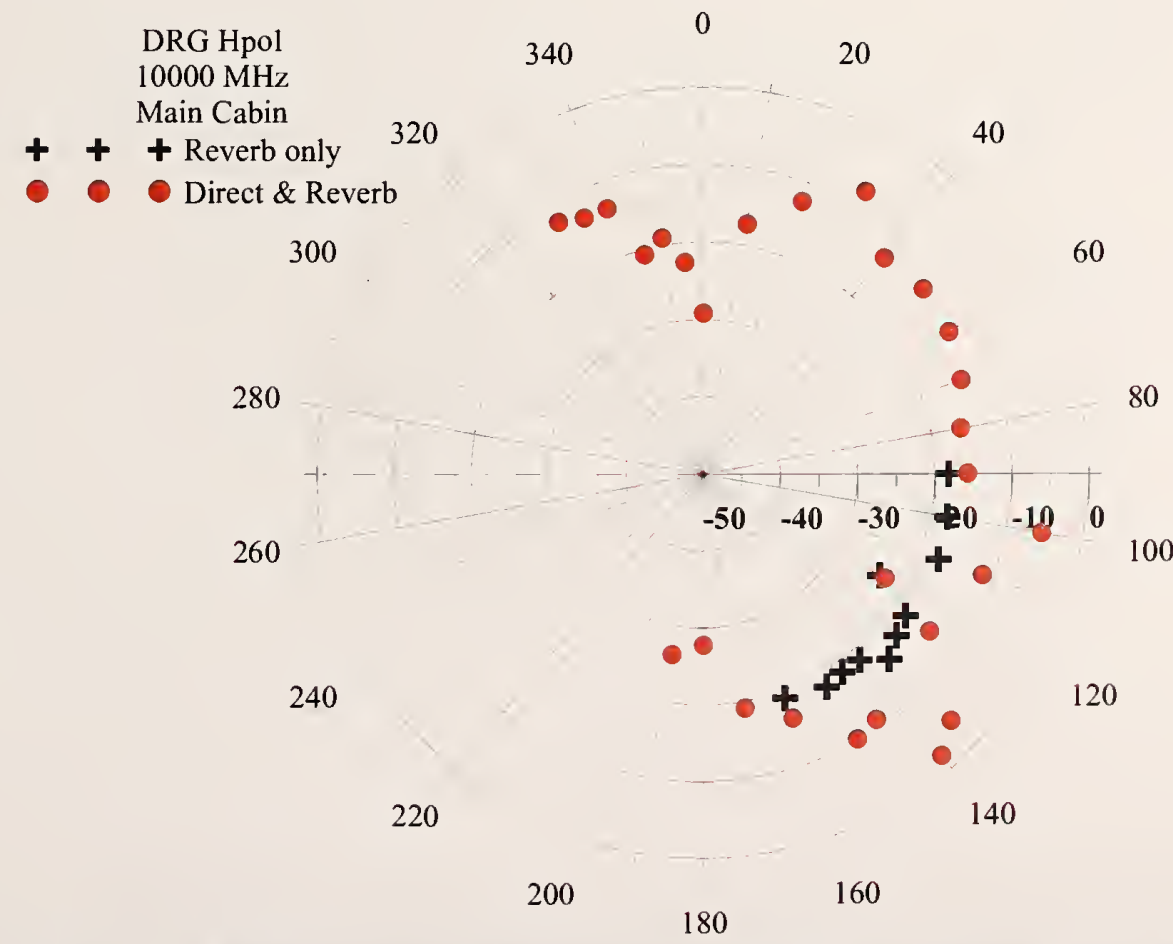

Figure 152. Penetration at $10000 \mathrm{MHz}$, for the DRG antennas in the vertical polarization, by computing only the reverberant component (black + ) vs. computing the direct plus reverberant component (red 9 ). 


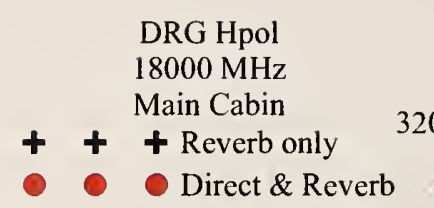

300

280

260

240 $\begin{array}{lll}340 & 0 & 20\end{array}$

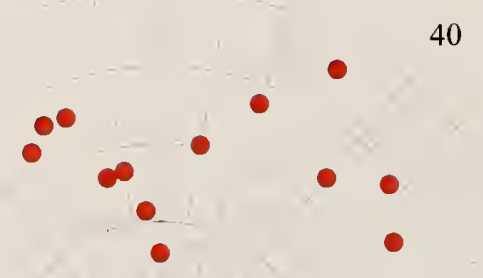

60

80

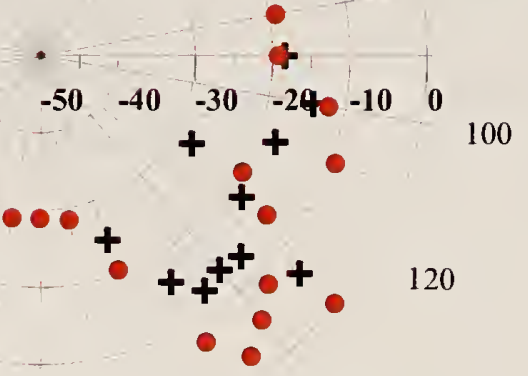

140

200

160

180

Figure 153. Penetration at $18000 \mathrm{MHz}$, for the DRG antennas in the horizontal polarization, by computing only the reverberant component (black +) vs. computing the direct plus reverberant component (red O).

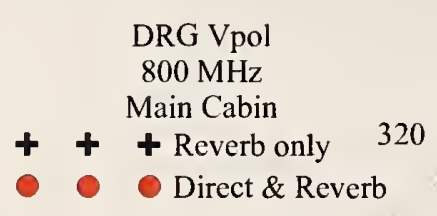

300

280

260

240
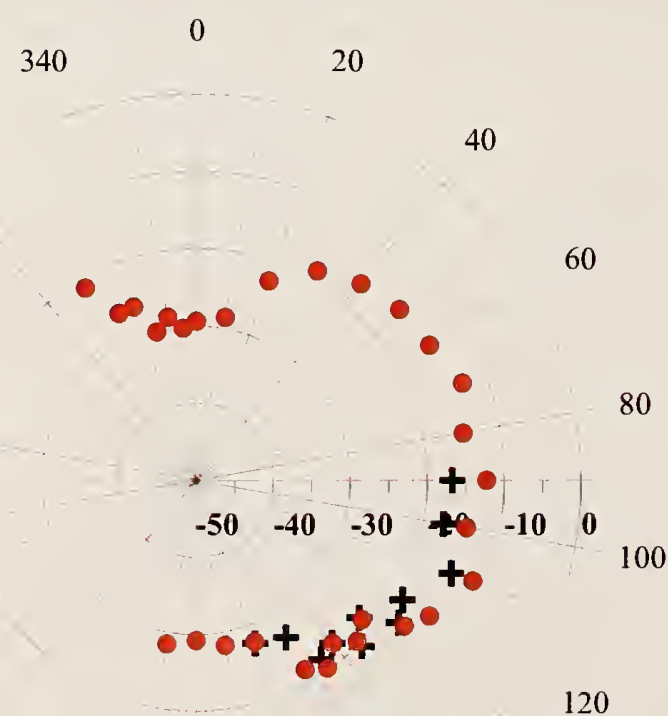

140

200

180

Figure 154. Penetration at $800 \mathrm{MHz}$, for the $\mathrm{DRG}$ antennas in the vertical polarization, by computing only the reverberant component (black $\boldsymbol{+}$ ) vs. computing the direct plus reverberant component (red $\bigcirc$ ). 


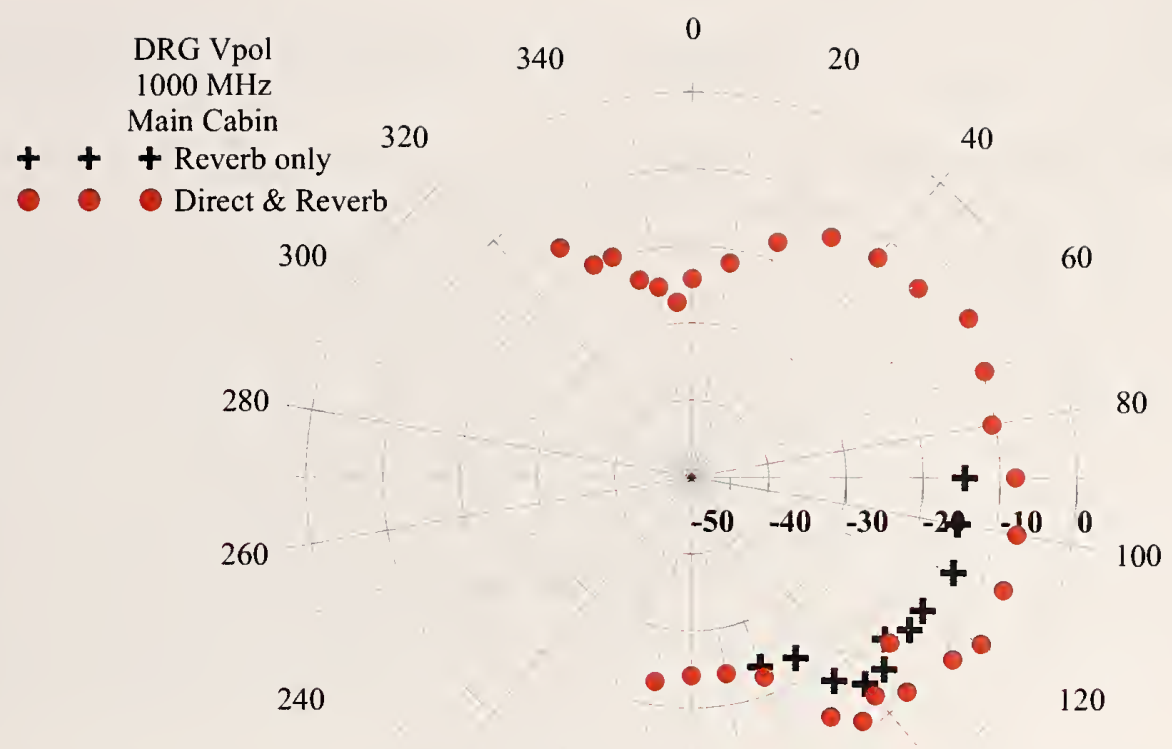

220

140

200

160

180

Figure 155. Penetration at $1000 \mathrm{MHz}$, for the DRG antennas in the vertical polarization, by computing only the reverberant component (black $\boldsymbol{t}$ ) vs. computing the direct plus reverberant component (red 0 ).

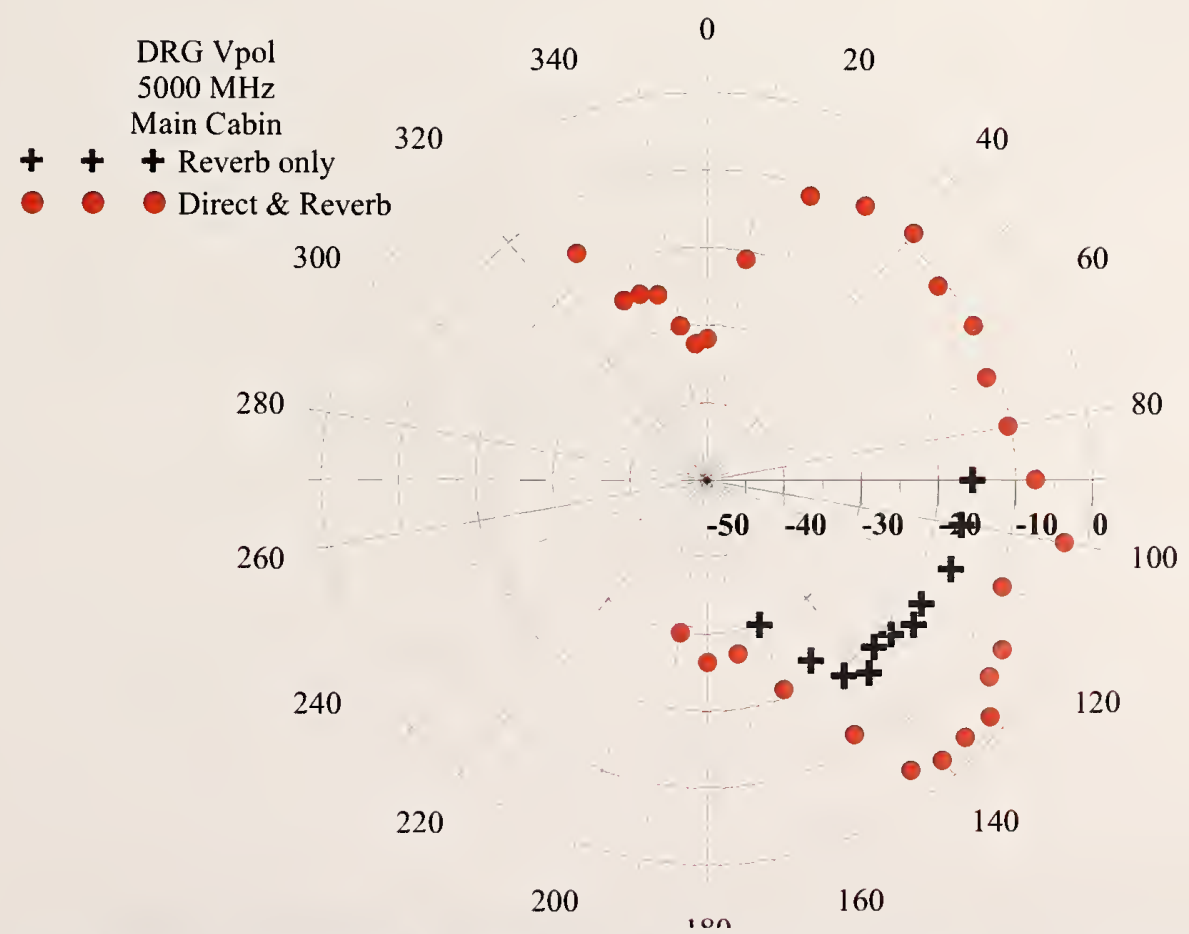

Figure 156. Penetration at $5000 \mathrm{MHz}$, for the DRG antennas in the vertical polarization, by computing only the reverberant component (black $\boldsymbol{+}$ ) vs. computing the direct plus reverberant component (red 0 ). 


\section{DRG Vpol $10000 \mathrm{MHz}$ \\ Main Cabin \\ +++ Reverb only \\ - O Direct \& Reverb}

300

240

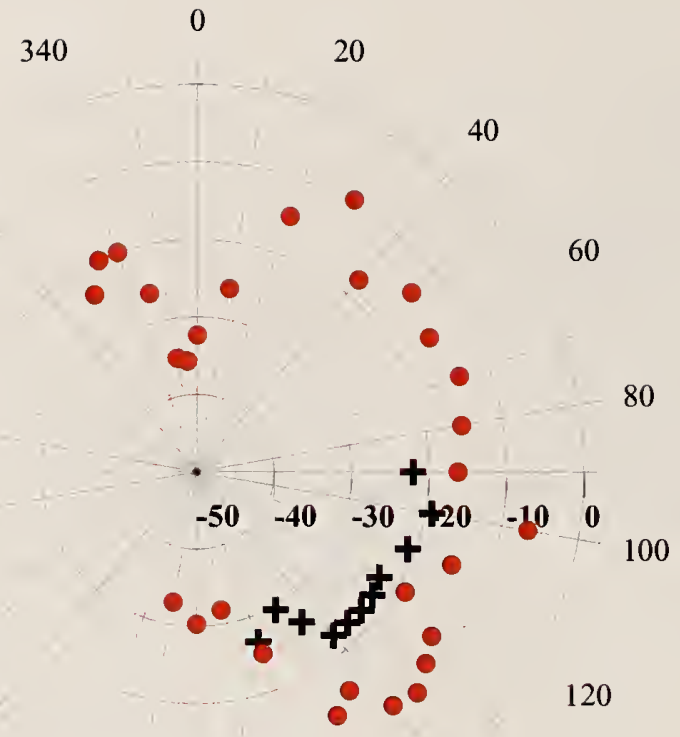

140

200

$180 \quad 160$

Figure 157. Penetration at $10000 \mathrm{MHz}$, for the DRG antennas in the vertical polarization, by computing only the reverberant component (black $\boldsymbol{+}$ ) vs. computing the direct plus reverberant component (red 0 ).

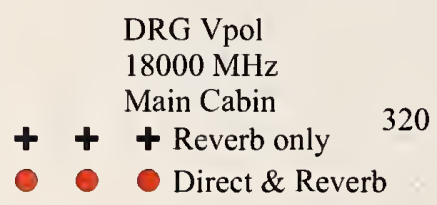

300

280

260

240

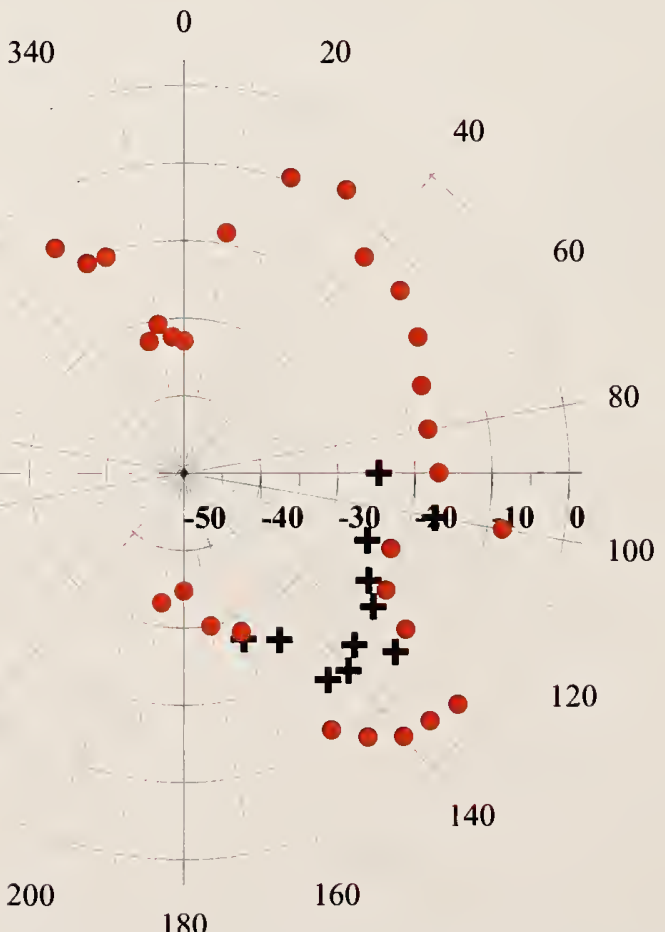

Figure 158. Penetration at $18000 \mathrm{MHz}$, for the DRG antennas in the vertical polarization, by computing only the reverberant component (black + ) vs. computing the direct plus reverberant component (red $\odot$ ). 


\section{Appendix B. Signal and Noise Characteristics}

Typical signal and noise characteristics are shown in Figures 159 to 161 for the three compartments: (1) main passenger cabin, (2) flight deck, and (3) cargo closet, at three different angular positions: (1) $30^{\circ}$, (2) $45^{\circ}$, and (3) $180^{\circ}$, respectively. We have plotted only the DRG horn antenna in the horizontal polarization for this section. Noise is a function of position due to the location of ambient RF sources which the aircraft may shield from the internal antennas as we move around the aircraft. Noise floors tend to be around $-60 \mathrm{~dB}$ to $-80 \mathrm{~dB}$ at the lower frequencies and climb to around $-50 \mathrm{~dB}$ at the higher frequencies. Noise levels peak at frequencies around $2000 \mathrm{MHz}$ due to external sources. The rise in the noise floor at around 9 $\mathrm{GHz}$ is due to the attenuator configuration of the VNA for the upper frequencies. The signal-tonoise ratios for the main passenger cabin vary from $30 \mathrm{~dB}$ to $60 \mathrm{~dB}$. In the flight deck, the signal-to-noise ratio varies from $20 \mathrm{~dB}$ to $55 \mathrm{~dB}$, and in the cargo closet, the signal-to-noise ratio varies from $15 \mathrm{~dB}$ to $55 \mathrm{~dB}$.

The signal-to-noise characteristics for the TEM horn antennas are a little different due to the FM frequency bands. Over the frequency band from $100 \mathrm{MHz}$ to $1500 \mathrm{MHz}$, typical signal-to-noise ratios range from $20 \mathrm{~dB}$ at the lower frequencies to $60 \mathrm{~dB}$ at the higher frequencies.

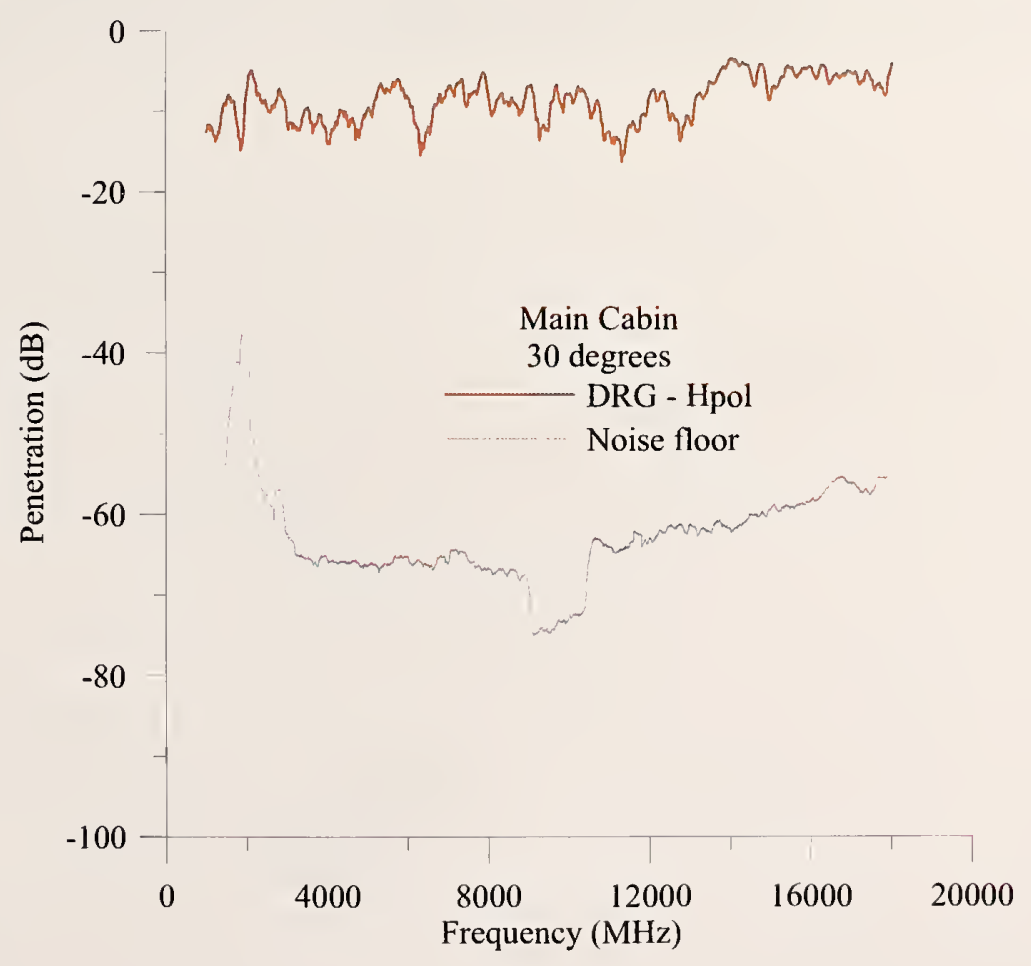

Figure 159. Typical signal and noise characteristics for measurements in the main passenger cabin. This particular noise measurement was taken at an angle of $30^{\circ}$. 


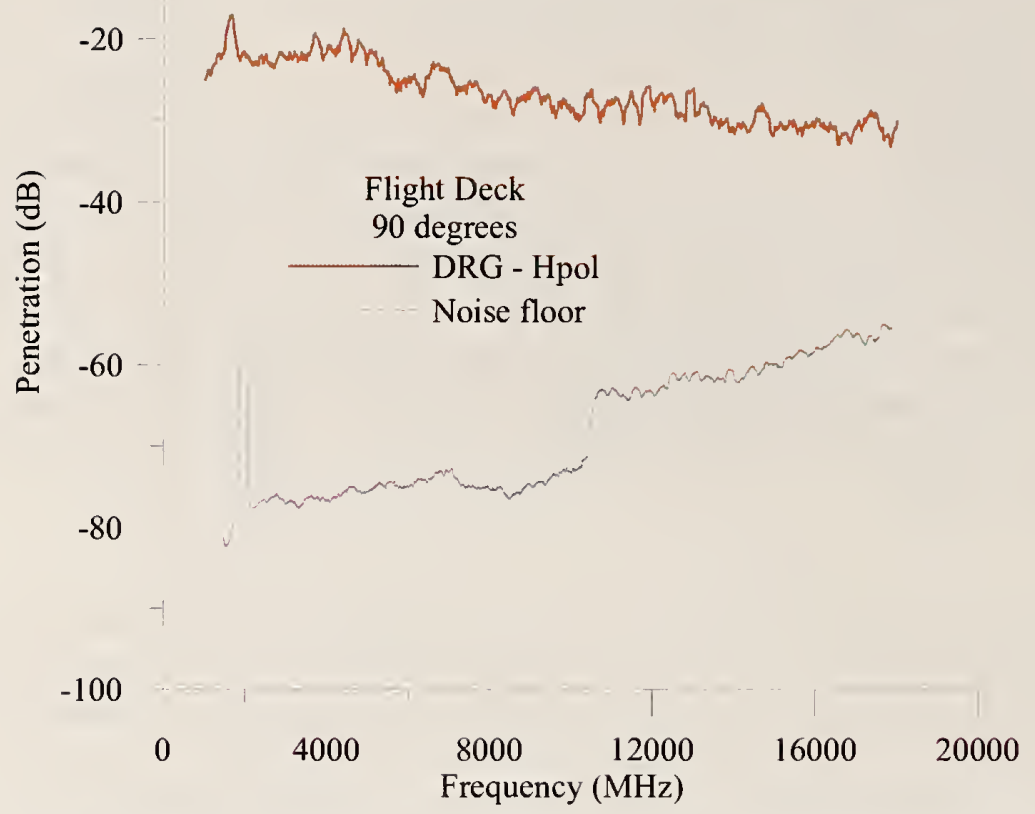

Figure 160. Typical signal and noise characteristics for measurements in the flight deck. This particular noise measurement was taken at an angle of $90^{\circ}$.

0

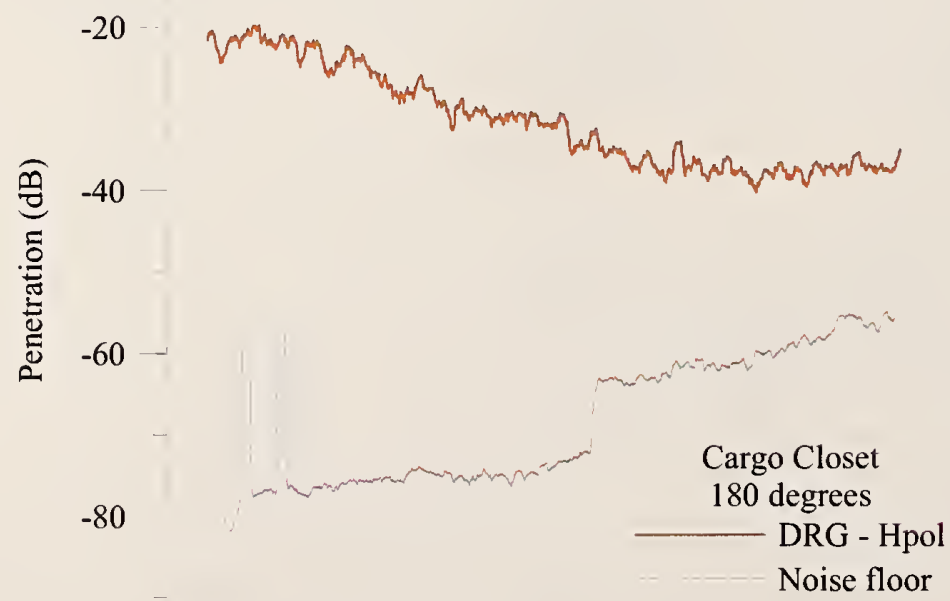

$-100$

$\begin{array}{cccccc}0 & 4000 & 8000 & 12000 & 16000 & 20000 \\ \text { Frequency }(\mathrm{MHz})\end{array}$

Figure 161. Typical signal and noise characteristics for measurements in the cargo closet. This particular noise measurement was taken at an angle of $180^{\circ}$. 


\section{Appendix C: TEM and DRG Horn Boresight Gain Characteristics}

The DRG and TEM horn antennas used in this effort were calibrated before we deployed our system in Atlantic City. This technique can be used to derive antenna gain with a specified resistive load (typically $50 \Omega$ ) at the antenna terminals. An accurate method for doing this is the three-antenna method. As the name implies, three transmission measurements are made with three combinations of the three antennas. This procedure is described in detail in reference [9]. This process yields three equations that can be readily solved for either gain or antenna factor. The gain characteristics of our DRG horn antennas are shown in Figure 162; those for our $36 \mathrm{~cm}$ TEM horns are shown in Figure 163; and those for our 1.2 m TEM horns are shown in Figure 164. The gains displayed in these figures include the antenna input mismatch, which accounts for the reduction of gain at the lower frequencies. The DRG horn antenna gain, plotted in Figure 162 shows that the low-frequency cutoff of these antennas occurs around $750 \mathrm{MHz}$. As the frequency is reduced, the input mismatch increases, which accounts for a rapid decrease in antenna gain. Mismatch and gain are not cause and effect, but a combination of both aperture beam forming and a better input match accounts for the increasing gain at the higher frequencies. A maximum gain of approximately $15 \mathrm{~dB}$ occurs at $16000 \mathrm{MHz}$. The $36 \mathrm{~cm}$ antenna results, plotted in Figure 163 show a maximum gain of approximately $7 \mathrm{~dB}$ realized at $4,000 \mathrm{MHz}$.

Results for a NIST $1.2 \mathrm{~m}$ horn antenna are plotted in Figure 164. This horn is longer, and it has a resistive taper, which improves the low-frequency performance. The maximum frequency of this antenna is $1500 \mathrm{MHz}$, due to balun performance limitations and a maximum gain of $7 \mathrm{~dB}$ occurs at this frequency as well. 


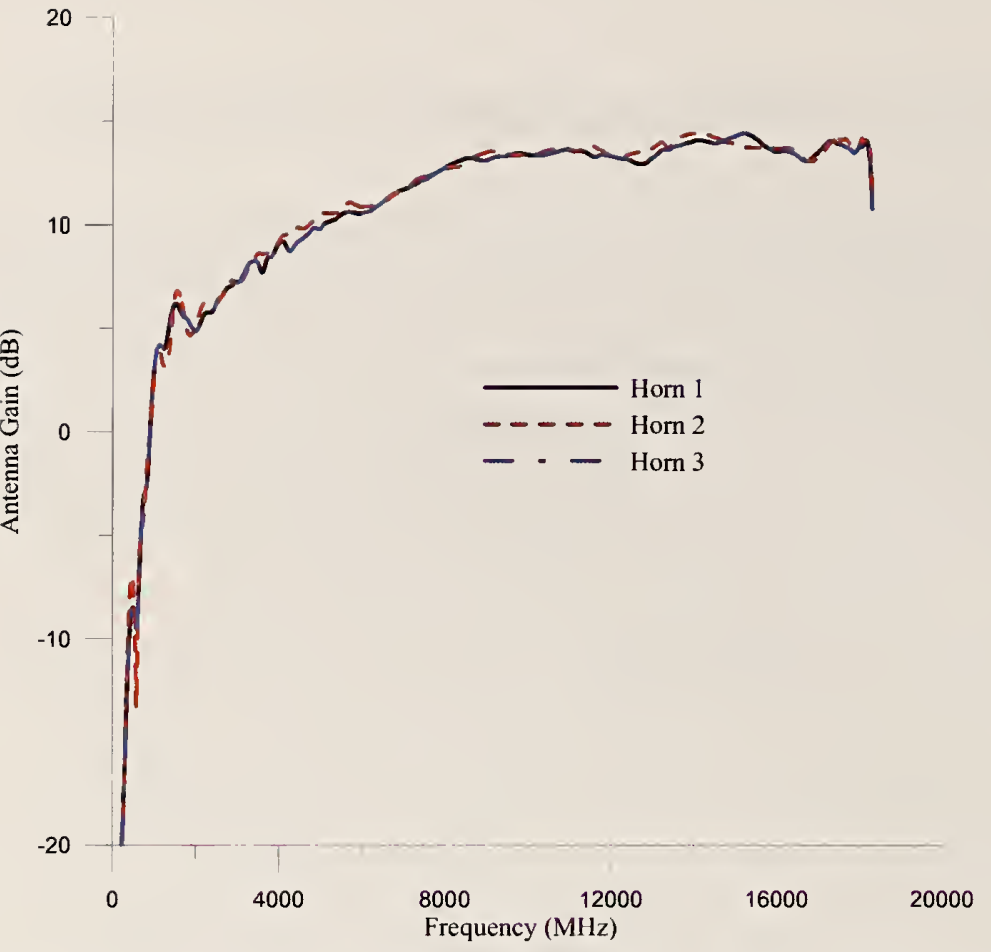

Figure 162. Measured boresight antenna gain for a 3117 DRG horn antenna. The gains plotted here include the input mismatch effects, which account for the rapid decrease below $750 \mathrm{MHz}$.

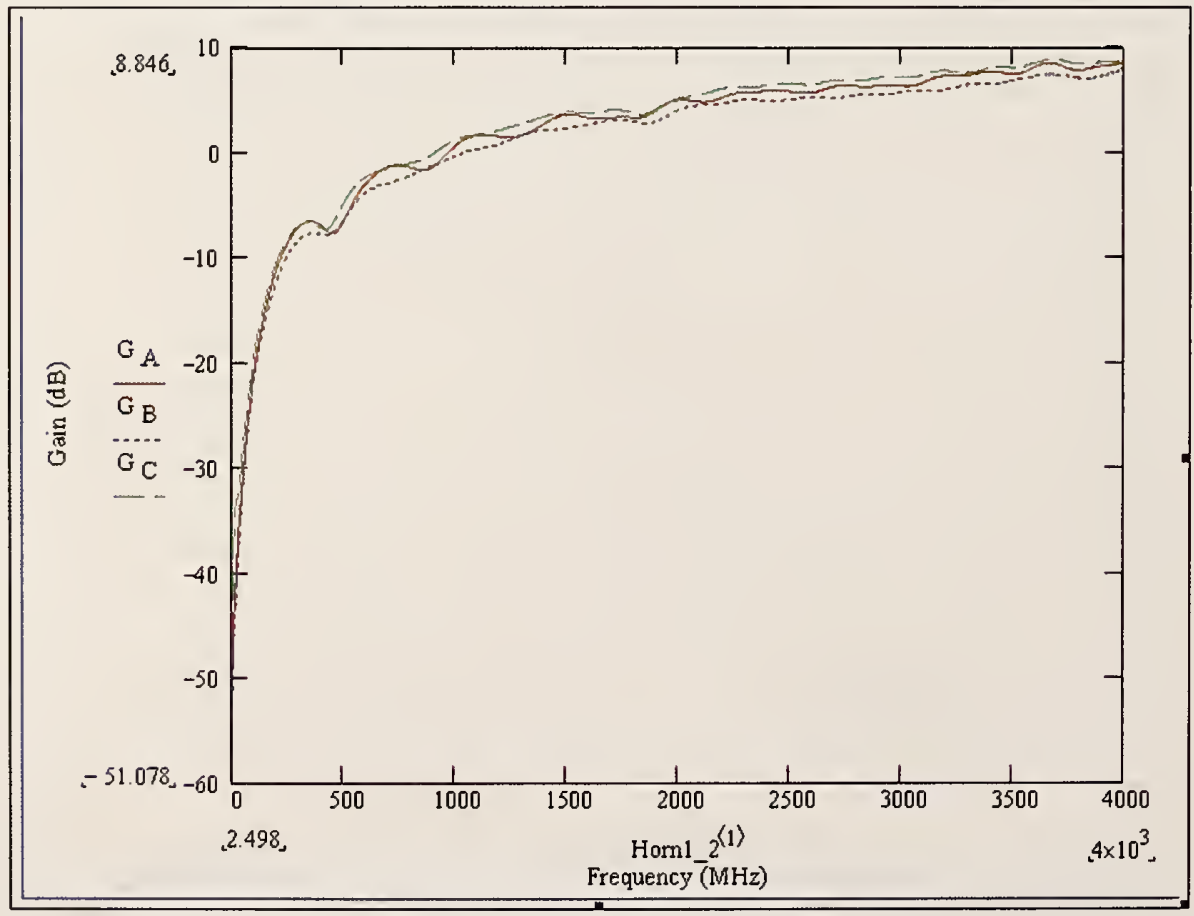

Figure 163. Measured boresight antenna gain for three identical NIST $36 \mathrm{~cm}$ TEM horn antennas. The gains plotted here include the input mismatch effects, which account for the rapid decrease below $100 \mathrm{MHz}$. 


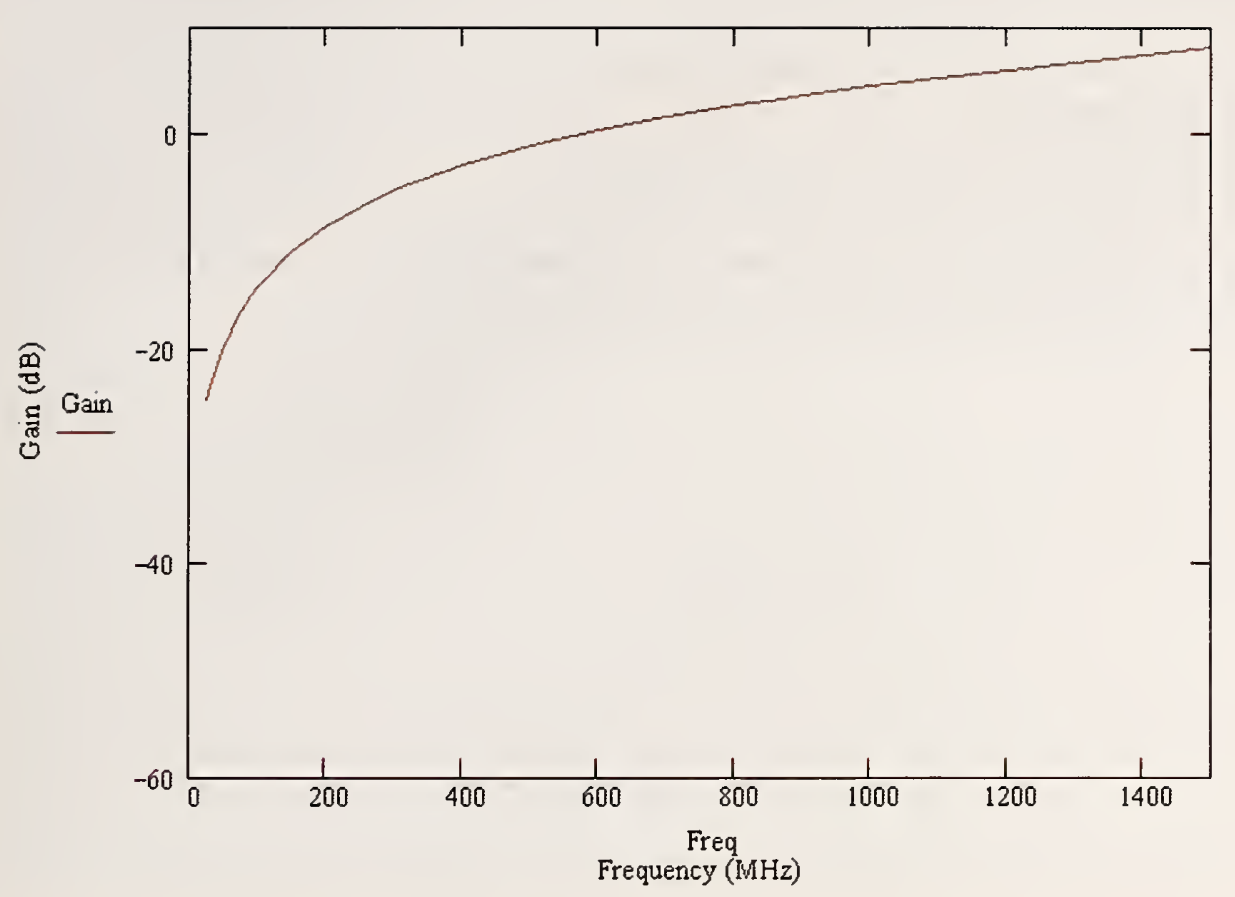

Figure 164. Measured boresight antenna gain for a NIST $1.2 \mathrm{~m}$ TEM horn antenna. The gains plotted here include the input mismatch effects, which account for the rapid decrease below $50 \mathrm{MHz}$.

\section{Appendix D: Penetration Data for HIRF Frequency Bands}

The peak and average field strengths in a HIRF environment are defined for specific frequency bands [1]. The frequency bands that correspond to our measured data are: (1) $200 \mathrm{MHz}$ to 400 $\mathrm{MHz}$, (2) $400 \mathrm{MHz}$ to $700 \mathrm{MHz}$, (3) $700 \mathrm{MHz}$ to $1000 \mathrm{MHz}$, (4) $1000 \mathrm{MHz}$ to $2000 \mathrm{MHz}$, (5) $2000 \mathrm{MHz}$ to $4000 \mathrm{MHz}$, (6) $4000 \mathrm{MHz}$ to $6000 \mathrm{MHz}$, (7) $6000 \mathrm{MHz}$ to $8000 \mathrm{MHz}$, (8) 8000 $\mathrm{MHz}$ to $12000 \mathrm{MHz}$, and (9) $12000 \mathrm{MHz}$ to $18000 \mathrm{MHz}$. If we determine the maximum penetration per compartment in these same frequency bands and plot it versus our measured data, this is likely of more interest to the HIRF community. Figures 165 to 167 are plots for the TEM and DRG antennas in the vertical and horizontal polarizations, at one angular position for each of the measured compartments. The black dotted line indicates the maximum penetration for that compartment.

Figure 165 shows the penetration data as a function of the HIRF environment frequency bands for the receiving antenna in the main cabin and the transmitting antenna at an angular position of $145^{\circ}$. This is an angle where there is high electromagnetic penetration into the aircraft. We notice that the maximum penetration is positive in certain frequency bands. Although the solution seems at first unrealistic, we have shown from our penetration definition that an aperture backed by a cavity can lead to a positive penetration value [10]. Penetration into the flight deck at an angle of $340^{\circ}$ is shown in Figure 166. Again, the black dotted line shows the maximum 
penetration for all angles as a function of the frequency band. We can see that the penetration into the aircraft at this angle is well below that of the maximum penetration for this cavity.

Finally, Figure 167 shows the penetration for the receiving antenna in the cargo closet, and the transmitting antenna at an angle of $50^{\circ}$. Most of the data in the lower frequency bands are well below the maximum penetration, and in the upper frequency bands we see that the penetration is closer to the maximum penetration for this compartment. If we use the maximum penetration for each compartment, we can then determine the peak and average field levels inside that compartment from the peak and average field levels outside of the aircraft. 

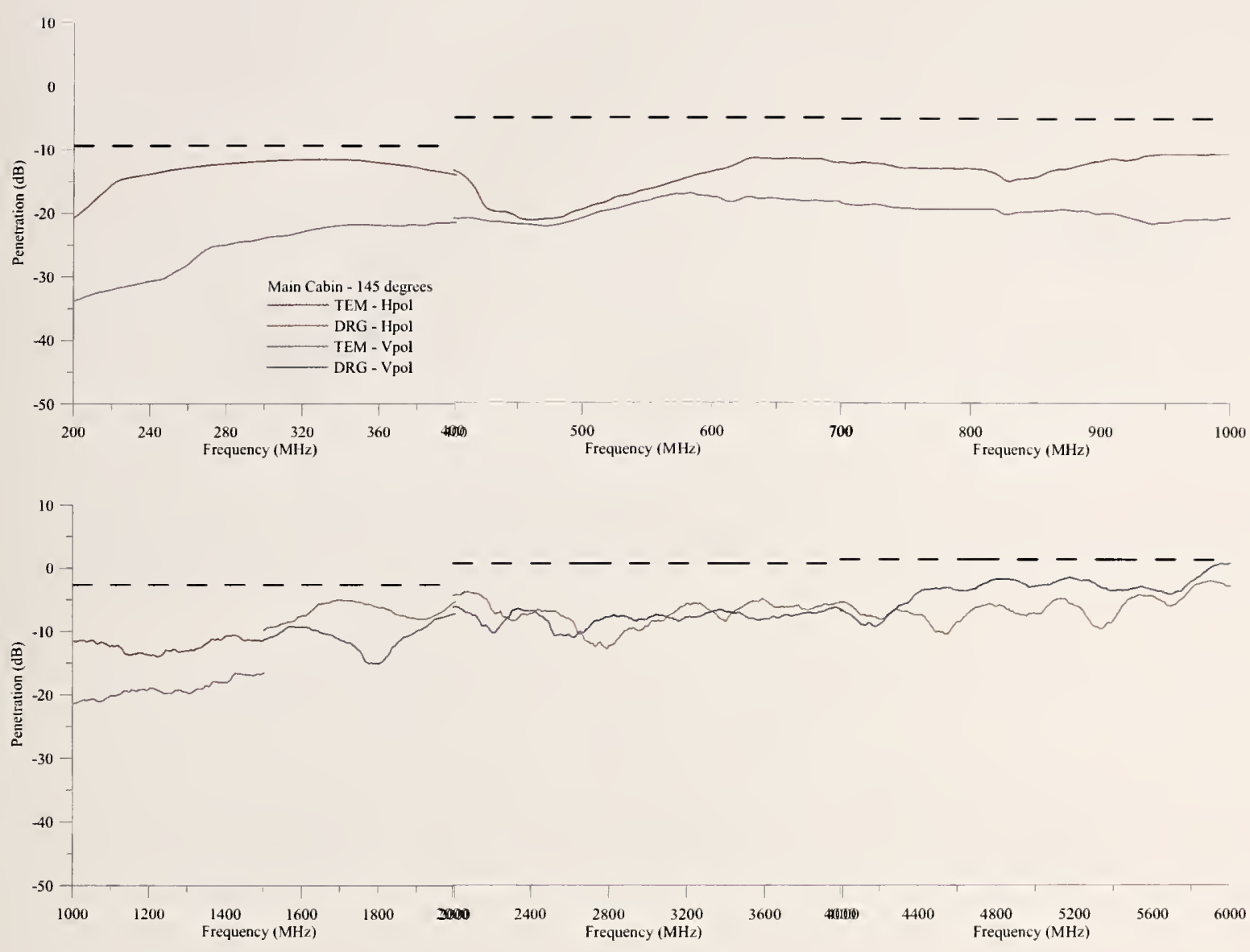

10

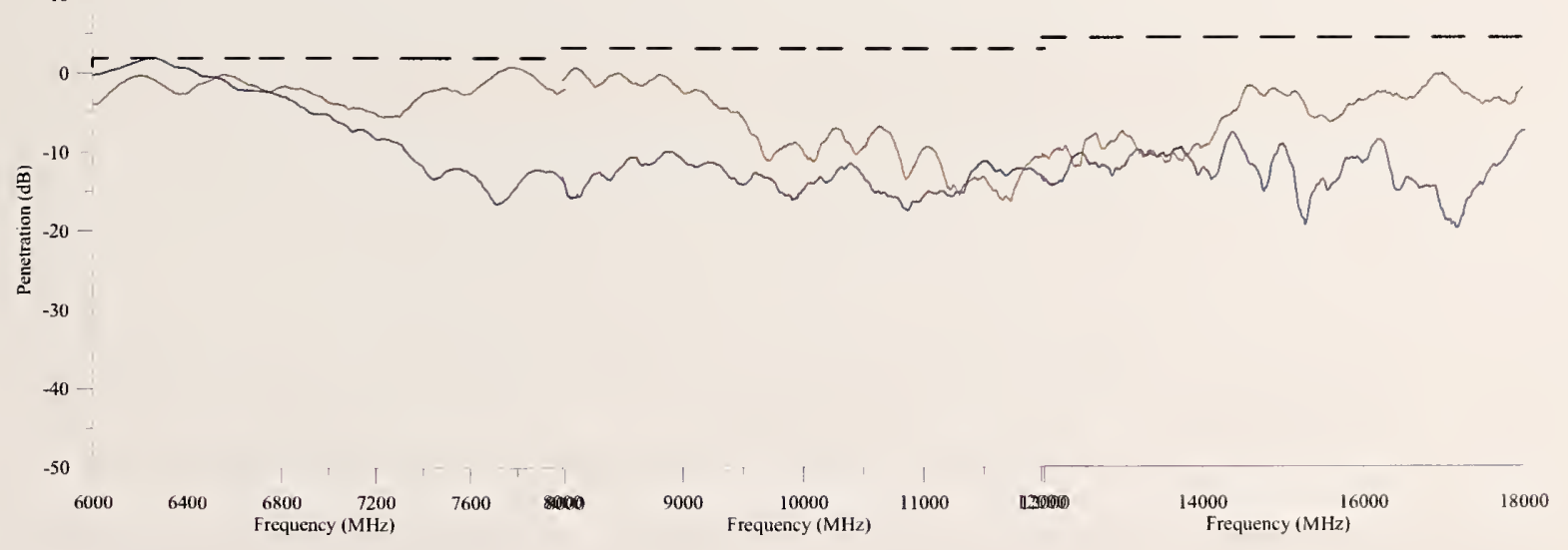

Figure 165. Frequency band exploded view of the penetration for all antennas and polarizations for the transmitting tower placed at the $145^{\circ}$ position and the receiver placed in the main cabin. 

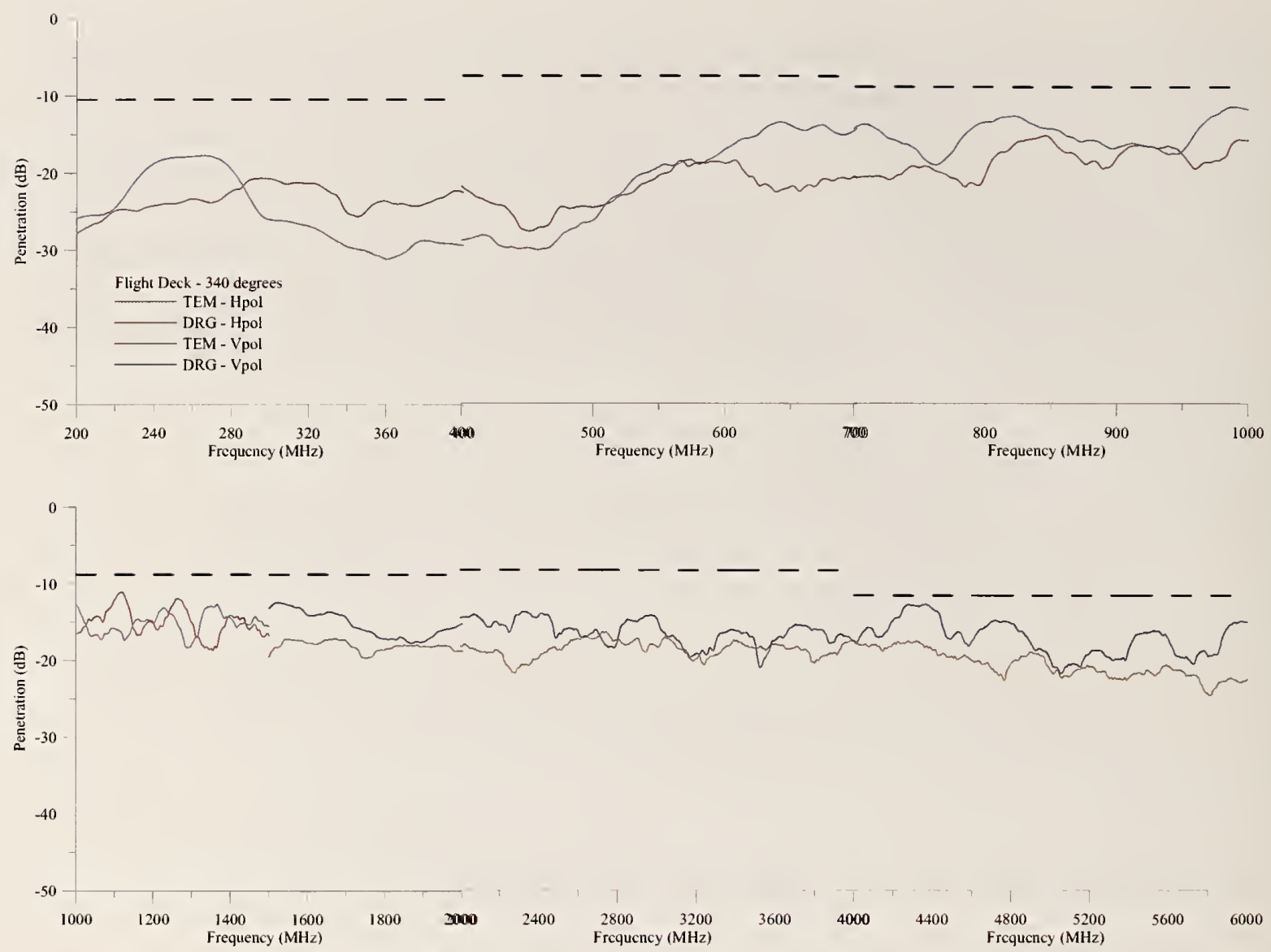

0

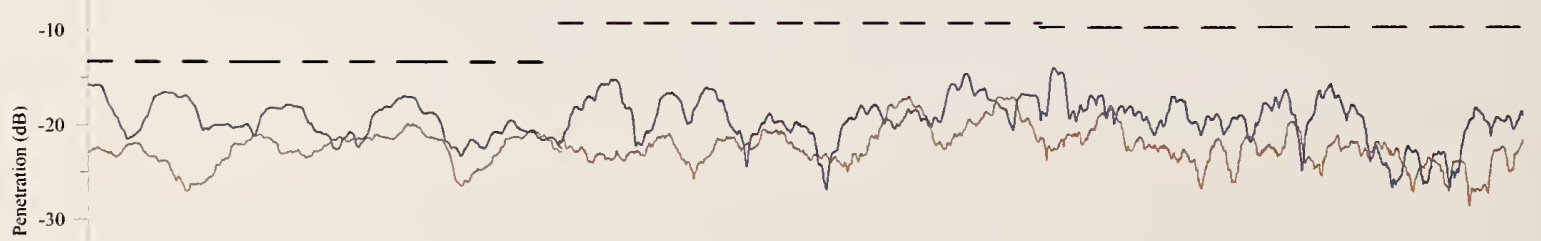

$-40$

$\begin{array}{rrrrrrrrrr}6000 & 6400 & 6800 & 7200 & 7600 & 9000 & 10000 & 11000 & 12000 & 14000 \\ \text { Frequency }(\mathrm{MHz})\end{array}$

Figure 166. Frequency band exploded view of the penetration for all antennas and polarizations for the transmitting tower placed at the $340^{\circ}$ position and the receiver placed in the flight deck. 

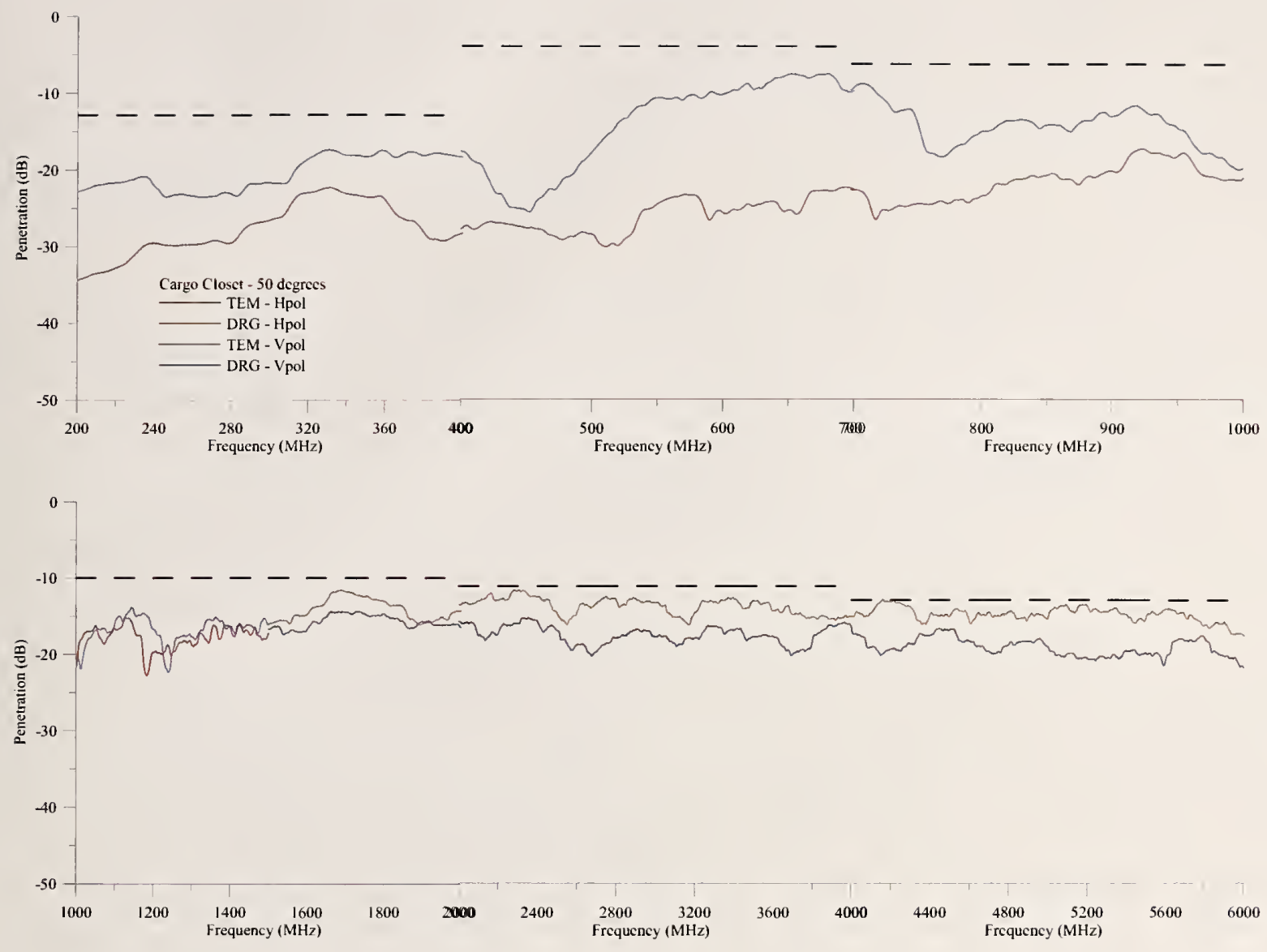

0

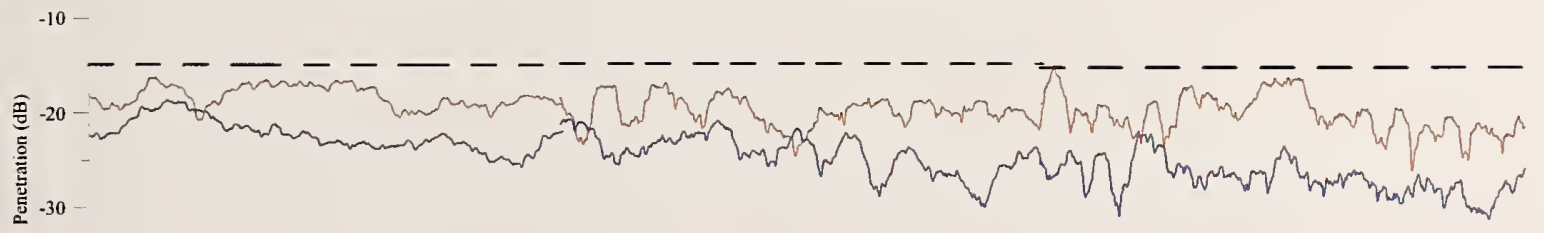

$40-$

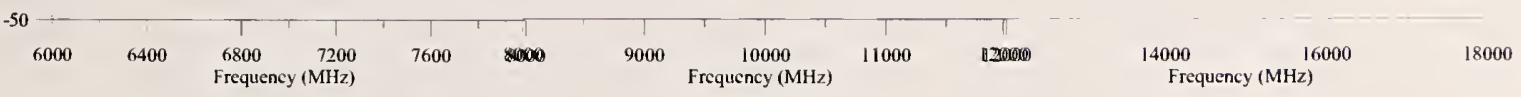

Figure 167. Frequency band exploded view of the penetration for all antennas and polarizations for the transmitting tower placed at the $50^{\circ}$ position and the receiver placed in the cargo closet. 


\section{Appendix E: Bombardier Global 5000 Measurement Equipment List}

The main equipment used in the evaluation of the Bombardier Global 5000 is listed below:

- Agilent N5230A PNA-L ( $300 \mathrm{kHz}$ to $18 \mathrm{GHz}$ ) with a maximum of 16001 frequencies/trace.

- Insulated Wire (IW) Cables (4 $\mathrm{m}$ to5 $\mathrm{m}$ )—we used phase-stabilized cables to avoid phase drift with temperature variations.

- Miteq Optical Fiber Link MDDR/MDDT (11 GHz version) - this unit has the ruggedness and temperature characteristics for field use.

- Miteq Optical Fiber Link SCMR/SCMT (18 GHz version) - this unit has the ruggedness and temperature characteristics for field use.

- HP 11713A Switch Controller and HP 3781 Switches

- HP 59306A Relay Actuator

- HP E3615A DC Power Supply for relay control

- AML 0120L2403 Amplifier for DRG antennas

- Harrison 6205 DC Power Supply

- Two 1.2 m TEM Horn Antennas designed and built at the NIST Boulder Laboratories

- One $36 \mathrm{~cm}$ TEM Horn Antenna designed and built at the NIST Boulder Laboratories

- Three 3117 DRG Horn Antennas

- NIST-developed "RADAR" Labview program used to perform time/frequency transformations, time gating, and signal processing

- $\quad$ NIST-developed "multical" Labview program used to perform multiband VNA calibrations and to circumvent the limitation of only 16001 frequency points. This program permits an arbitrarily large number of frequency points. In practice we typically use as many as 48003 points.

\footnotetext{
* Certain commercial equipment, instruments, or materials are identified in this paper in order to adequately specify the experimental procedure. Such identification does not imply recommendation or endorsement by the National Institute of Standards and Technology, nor does it imply that the materials or equipment identified are necessarily the best available for the purpose.
} 


\section{NIST Technical Publications}

\section{Periodical}

Journal of Research of the National Institute of Standards and Technology-Reports NIST research and development in metrology and related fields of physical science. engineering, applied mathematics, statistics. biotechnology, and information technology. Papers cover a broad range of subjects, with major emphasis on measurement methodology and the basic technology underlying standardization. Also included from time to time are survey articles on topics closely related to the Institute's technical and scientific programs. Issued six times a year.

\section{Nonperiodicals}

Monographs-Major contributions to the technical literature on various subjects related to the Institute's scientific and technical activities.

Handbooks-Recommended codes of engineering and industrial practice (including safety codes) devel- oped in cooperation with interested industries, professional organizations, and regulatory bodies.

Special Publications-Include proceedings of conferences sponsored by NIST, NIST annual reports, and other special publications appropriate to this grouping such as wall charts, pocket cards. and bibliographies.

National Standard Reference Data Series-Provides quantitative data on the physical and chemical properties of materials, compiled from the world's literature and critically evaluated. Developed under a worldwide program coordinated by NIST under the authority of the National Standard Data Act (Public Law 90-396). NOTE: The Journal of Physical and Chemical Reference Data (JPCRD) is published bimonthly for NIST by the American Institute of Physics (AIP). Subscription orders and renewals are available from AIP. P.O. Box 503284. St. Louis, MO 63150-3284.

Building Science Series-Disseminates technical information developed at the Institute on building materials. components, systems, and whole structures. The series presents research results, test methods, and performance criteria related to the structural and environmental functions and the durability and safety characteristics of building elements and systems.

Technical Notes-Studies or reports which are complete in themselves but restrictive in their treatment of a subject. Analogous to monographs but not so comprehensive in scope or definitive in treatment of the subject area. Often serve as a vehicle for final reports of work performed at NIST under the sponsorship of other government agencies. Voluntary Product Standards-Developed under procedures published by the Department of Commerce in Part 10 , Title 15, of the Code of Federal Regulations. The standards establish nationally recognized requirements for products, and provide all concerned interests with a basis for common understanding of the characteristics of the products. NIST administers this program in support of the efforts of private-sector standardizing organizations.

Order the following NIST publications-FIPS and NISTIRs-from the National Technical Information Service. Springfield. I'A 22161.

Federal Information Processing Standards Publications (FIPS PUB)-Publications in this series collectively constitute the Federal Information Processing Standards Register. The Register serves as the official source of information in the Federal Government regarding standards issued by NIST pursuant to the Federal Property and Administrative Services Act of 1949 as amended. Public Law 89-306 (79 Stat. I I27), and as implemented by Executive Order I1717 (38 FR I2315, dated May I1.1973) and Part 6 of Title I5 CFR (Code of Federal Regulations).

NIST Interagency or Internal Reports (NISTIR)-The series includes interim or final reports on work performed by NIST for outside sponsors (both government and nongovernment). In general, initial distribution is handled by the sponsor; public distribution is handled by sales through the National Technical Information Service, Springfield, VA 2216I, in hard copy, electronic media, or microfiche form. NISTIR's may also report results of NIST projects of transitory or limited interest. including those that will be published subsequently in more comprehensive form. 
U.S. Department of Commerce

National Bureau of Standards and Technology

325 Broadway

Boulder, CO 80305-3328

\section{Official Business}

Penalty for Private Use $\$ 300$ 